Marcelo Lipas Augusto

\title{
Contribuição para a análise de teletráfego com dependência de longa duração
}

\author{
Dissertação apresentada à Escola Poli- \\ técnica da Universidade de São Paulo \\ para obtenção do Título de Mestre em \\ Engenharia Elétrica.
}


Marcelo Lipas Augusto

\section{Contribuição para a análise de teletráfego com dependência de longa duração}

Dissertação apresentada à Escola Politécnica da Universidade de São Paulo para obtenção do Título de Mestre em Engenharia Elétrica.

Área de concentração:

Sistemas Eletrônicos

Orientador:

Prof. Dr. José Roberto de Almeida Amazonas 
Para minha esposa Kate e nossa filha Giovanna. 


\section{Agradecimentos}

A Deus, por todas as inúmeras graças em minha vida.

Ao meus pais Jorge e Irene, por todo o amor e dedicação colocados em minha educação.

À minha esposa Kate, pelo seu amor verdadeiramente incondicional.

Ao meu orientador Prof. Dr. José Roberto Amazonas, pela confiança depositada em meu trabalho.

Ao amigo Prof. Dr. Alexandre Barbosa de Lima, por todo o suporte sem o qual não seria possível a conclusão deste trabalho.

Aos meus colegas e amigos que, direta ou indiretamente, contribuíram ao longo de toda a pesquisa e ao longo da redação do texto desta dissertação. 


\section{Resumo}

A utilização de modelos de teletráfego que contemplem características tais como autossimilaridade e dependência de longa duração tem se mostrado cada vez mais como sendo ponto-chave na correta caracterização do teletráfego Local Area Network (LAN) e Wide Area Network (WAN) [1, 2]. Tal caracterização é necessária para o monitoramento e controle de teletráfego em redes convergentes [3]. Nesse contexto, a questão da estimação precisa do parâmetro de autossimilaridade, denominado de parâmetro de Hurst, torna-se essencial. Entretanto, estudos comprovam que, além da dependência de longa duração, redes WAN podem, não raramente, apresentar características mistas de dependência de longa e de curta duração $[4,5]$. Enquanto vasta literatura científica, tanto teórica como prática, tem abordado com afinco a questão da acurácia de diversos estimadores para o parâmetro de Hurst $[6,7,8,9]$, pouca atenção tem sido dada à questão da estimação deste parâmetro na presença de dependência de curta duração.

O presente trabalho de pesquisa concentrou-se no estudo dos métodos de estimação do parâmetro de Hurst baseados no espectro wavelet, em particular através do método de Abry-Veitch [10] - baseado na transformada Discrete Wavelet Transform (DWT) - e através do espectro obtido através da transformada Discrete Wavelet Packet Transform (DWPT). Os resultados baseados no método de Abry-Veitch demonstram que, através de um ajuste apropriado dos parâmetros de estimação, tal método permite uma estimação robusta na presença de componentes com dependência de curta duração, mesmo em situações de mudança de regime de tal componente, característica desejável para a estimação em tempo real do parâmetro de Hurst. Entretanto, a dispersão considerável apresentada, em alguns casos, pelas estimativas do método de Abry-Veitch, motivou o estudo da utilização do espectro wavelet obtido via transformada DWPT para realização da estimação do parâmetro de Hurst. Os resultados indicam que a utilização de tal transformada gera um espectro wavelet tal que é possível detectar a presença ou não de componentes com dependência de curta duração.

Ao final, os resultados da pesquisa realizada são sumarizados e utilizados em uma proposta de mecanismo de estimação do parâmetro de Hurst em tempo real, na presença simultânea de componentes de dependência de longa e curta duração. 


\section{Abstract}

The use of network traffic models that hold self-similar and long-range dependence characteristics have shown to be a key element on the correct characterization of Local Area Network (LAN) and Wide Area Network (WAN) network traffic [1, 2]. Such characterization is necessary to monitor and control the network traffic in converged networks [3]. In this context, the accurate estimation of the selfsimilarity parameter, named Hurst parameter, is a major issue. However, studies show that, besides the long-range dependence, WAN network traffic may, not uncommonly, present mixed long and short-range dependence characteristics [4, 5]. While great part of either theoretical or practical scientific literature has been focused on the issue of Hurst parameter estimator accuracy [6, 7, 8, 9], little attention has been given to the estimation of such parameter in the presence of short-range dependence.

This research work has focused on the study of the Hurst parameter estimation methods based on the wavelet spectrum, specially through the Abry-Veitch method [10] - which is based on the Discrete Wavelet Transform (DWT) transform - and through the wavelet spectrum based on the Discrete Wavelet Packet Transform (DWPT) transform. The results based on the Abry-Veitch method show that, through a suitable adjustment of the estimation parameters, such method yields a robust estimation in the presence of short-range dependence components, even in changing conditions of such component, a desirable characteristic for the real-time estimation of the Hurst parameter. However, the significant dispersion presented, occasionally, by the Abry-Veitch method estimates motivated the research of the usage of the wavelet spectrum obtained via DWPT transform to estimate the Hurst parameter. The results show that the usage of such transform generates such a wavelet spectrum that it is possible to detect whether short-range dependence components are present, or not, in the analyzed series.

At the end, the research results are summarized and used to propose a realtime Hurst parameter estimation mechanism, in the presence of simultaneous long- and short-range dependence components. 


\title{
Sumário
}

\section{Lista de Figuras}

\author{
Lista de Tabelas
}

\section{Lista de Abreviaturas}

1 Introdução 1

1.1 Motivação . . . . . . . . . . . . . . . . . . . . . 1

1.2 Objetivos e contribuições . . . . . . . . . . . . . . . . 4 4

1.3 Estrutura da dissertação . . . . . . . . . . . . . . . . . . . . 6

$\begin{array}{lll}\text { I Fundamentação teórica } & 7\end{array}$

$\begin{array}{llr}2 & \text { A natureza fractal do teletráfego } & 8\end{array}$

2.1 Fractais . . . . . . . . . . . . . . . 8

2.2 Dependência de longa duração . . . . . . . . . . . . . . . . . . 12

2.3 Autossimilaridade . . . . . . . . . . . . . . . . . 15

$\begin{array}{llr}3 & \text { Wavelets } & 17\end{array}$

3.1 Introdução . . . . . . . . . . . . . . . . . . . . . 17

3.2 Transformada DWT . . . . . . . . . . . . . . . . . . . 18

3.2.1 Análise Multirresolução . . . . . . . . . . . . . . . . 20

3.3 Transformada DWPT . . . . . . . . . . . . . . . . . . 23

3.3.1 Determinação da árvore DWPT . . . . . . . . . . . . . 27

3.4 Coeficientes wavelet de processos auto-similares e LRD . . . . . . 29 
4.1 Ruído Gaussiano fracionário . . . . . . . . . . . . . . . . . . 32

4.2 Métodos clássicos de estimação do parâmetro $H$. . . . . . . . . . 34

$4.2 .1 \quad$ Estatística $\mathrm{R} / \mathrm{S} \ldots \ldots \ldots \ldots . \ldots . \ldots 34$

4.2.2 Variance Plot . . . . . . . . . . . . . 35

4.2.3 Método do Periodograma . . . . . . . . . . . . . 35

4.2.4 Método de Whittle . . . . . . . . . . . . 36

4.3 Método wavelet de Abry-Veitch . . . . . . . . . . . . . . 36

4.3.1 Diagramas em escala logarítmica . . . . . . . . . 37

4.3.2 Estimação com diagramas em escala logarítmica . . . . . . 38

4.3 .3 Estimação de LRD . . . . . . . . . . . . . . . . . . 40

4.3.4 Análise de dados discretos . . . . . . . . . . . . . . 41

II Análise exploratória $\quad 43$

5 Objetivos 44

6 Método de Abry-Veitch 46

6.1 Análise preliminar ... . . . . . . . . . . . 46

6.1.1 Condições experimentais . . . . . . . . . . . . . 46

6.1.2 Resultados obtidos . . . . . . . . . . . . . 48

6.1.3 Exploração dos resultados . . . . . . . . . . . . . 56

6.1 .4 Síntese dos resultados . . . . . . . . . . . . 69

6.2 Análise da influência dos parâmetros de estimação do Método de

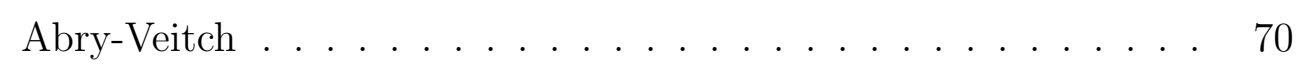

6.2.1 Condições experimentais . . . . . . . . . . . . . . 70

6.2.2 Resultados obtidos . . . . . . . . . . . . . 70

6.2 .3 Exploração dos resultados . . . . . . . . . . . . . 78

6.2 .4 Síntese dos resultados . . . . . . . . . . . . . 88 
6.3 Análise da influência da variação do nível de SRD . . . . . . . . . 89

6.3.1 Condições experimentais . . . . . . . . . . . . . . . 89

6.3.2 Resultados obtidos . . . . . . . . . . . . . . . 90

6.3.3 Exploração dos resultados . . . . . . . . . . . . . . . . 103

6.3.4 Síntese dos resultados . . . . . . . . . . . . . 107

6.4 Análise comparativa detalhada com resultados obtidos por Abry-

Veitch ......................... 108

6.4.1 Condições experimentais . . . . . . . . . . . . . . . 108

6.4.2 Resultados obtidos . . . . . . . . . . . . . . . . 109

6.4.3 Exploração dos resultados . . . . . . . . . . . . . . . . 112

6.4.4 Síntese dos resultados . . . . . . . . . . . . . . . . 128

$\begin{array}{llr}7 & \text { DWPT } & 129\end{array}$

7.1 Condições experimentais . . . . . . . . . . . . . . . . . . . . 129

7.2 Resultados obtidos . . . . . . . . . . . . . . . . . . . . 129

7.3 Exploração dos resultados . . . . . . . . . . . . . . . . . . . 139

7.4 Síntese dos resultados . . . . . . . . . . . . . . . . . . 147

8 Proposta de mecanismo de estimação 148

8.1 Fundamentação experimental . . . . . . . . . . . . . . . 148

8.2 Mecanismo de estimação em tempo real . . . . . . . . . . . . . . 150

8.3 Questões em aberto . . . . . . . . . . . . . . . . . . 152

$\begin{array}{lc}\text { III Conclusões } & 153\end{array}$

9 Conclusões e trabalhos futuros $\quad 154$

$\begin{array}{ll}\text { Referências } & 157\end{array}$

Apêndice A - Pseudocódigo detalhado do mecanismo de estimação proposto 


\section{Lista de Figuras}

1.1 Observações temporais e do espectro wavelet da série UNC-1 [30]. 3

1.2 Observações temporais e do espectro wavelet da série UNC-2 [30]. 3

1.3 Periodograma da série UNC-2 [30]. . . . . . . . . . . . . . 4

2.1 Exemplo de visualização do conjunto de Mandelbrot (ou boneco de pão-de-mel). . . . . . . . . . . . . . . . . . . 99 9

2.2 Fotos que revelam a característica autossimilar do contorno do litoral da península Escandinava [48] . . . . . . . . . . . . . . . . . 10

2.3 Foto que ilustra a característica autossimilar de uma couve-flor [48]. 10

2.4 Tráfego Fast Ethernet coletado em um servidor da Universidade de Drexel, apresentado em 4 diferentes níveis de agregação [50]. 11

2.5 Comparação das características impulsivas, em diversas escalas de agregação, do tráfego Ethernet real e sintetizado [4] . . . . . . . . 12

3.1 Exemplo de wavelets obtidas das derivadas da Probability Density Function (PDF) Gaussiana [3]. . . . . . . . . . . . . . . . . . . . 19

3.2 Diagrama de fluxo para a decomposição DWT [65]. . . . . . . . . 23

3.3 Diagrama de fluxo para a decomposição DWPT [65]. . . . . . . . 24

3.4 Comparação entre decomposições DWT (esq.) e DWPT (dir.) de um mesmo sinal $[66] \ldots \ldots \ldots \ldots$. . . . . . . . . . . . . 25

3.5 Wavelet packets de Haar [66]. . . . . . . . . . . . . 26

3.6 Wavelet packets $\mathrm{db} 2[66] \ldots \ldots \ldots 26$

3.7 Exemplo de escolhas de árvores DWPT para análise de um sinal [56]. 28

4.1 LD exato e estimativas do LD para um processo FGN com $H=$ $0,8[10] \ldots \ldots \ldots \ldots \ldots$

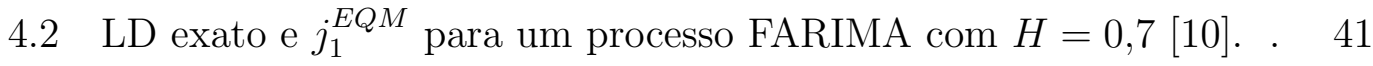

6.1 Análise espectral das séries originais sem SRD . . . . . . . . . . . 49 
6.2 Resposta em frequência dos filtros IIR utilizados para adição de Short-Range Dependence (SRD). . . . . . . . . . . . . . 50

6.3 Análise espectral das séries com SRD (filtragem com $f_{0} \approx 0,2$ ). . $\quad 51$

6.4 Análise espectral das séries com SRD (filtragem com $f_{0} \approx 0,4$ ). . . 52

6.5 Análise espectral das séries com SRD (filtragem com $f_{0} \approx 0,7$ ). . . 53

6.6 Efeitos da estimativa de $H$ em séries sem e com SRD. . . . . . . 55

6.7 Histograma e QQ-plot $\hat{H}$ para diversos valores de $j_{\min }$ (séries originais sem SRD). . . . . . . . . . . . . . . . . . . 58

6.8 Estatísticas de $\hat{H}$ para diversos valores de $j_{\text {min }}$ (séries originais sem

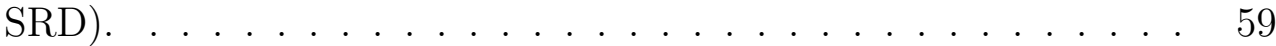

6.9 Histograma e QQ-plot para diversos valores de $j_{\min }$ (séries com $\mathrm{SRD}-f_{0} \approx 0,2 \pi \mathrm{rad} /$ amostra $) . \ldots . . . . . .$.

6.10 Estatísticas de $\hat{H}$ para diversos valores de $j_{\text {min }}$ (séries com SRD $f_{0} \approx 0,2 \pi \mathrm{rad} /$ amostra $) \ldots \ldots \ldots \ldots$

6.11 Histograma e QQ-plot para diversos valores de $j_{\min }$ (séries com $\mathrm{SRD}-f_{0} \approx 0,4 \pi \mathrm{rad} /$ amostra .

6.12 Estatísticas de $\hat{H}$ para diversos valores de $j_{\text {min }}$ (séries com SRD $f_{0} \approx 0,4 \pi \mathrm{rad} /$ amostra $) \ldots \ldots \ldots \ldots$

6.13 Histograma e QQ-plot para diversos valores de $j_{\min }$ (séries com $\mathrm{SRD}-f_{0} \approx 0,7 \pi \mathrm{rad} /$ amostra $)$.

6.14 Estatísticas de $\hat{H}$ para diversos valores de $j_{\text {min }}$ (séries com SRD $f_{0} \approx 0,7 \pi \mathrm{rad} /$ amostra). . . . . . . . . . . . . 65

6.15 Estimativas considerando-se todos os possíveis valores de $j_{\min }$. . .

6.16 Estimativas considerando-se todos os possíveis valores de $j_{\min }$ excluindo-se os outliers. . . . . . . . . . . . . . . . . 67

6.17 Comparação do espectro wavelet entre séries sem e com SRD. . . 68

6.18 Estimativas pelo método de Abry-Veitch em função de $H_{\text {gen }}$ para $j_{\text {min }} \in\left\{1, j_{\text {min }}^{E Q M}\right\}$ e $N=256 \ldots \ldots \ldots \ldots$

6.19 Estimativas pelo método de Abry-Veitch em função de $H_{\text {gen }}$ para $j_{\min } \in\left\{1, j_{\min }^{E Q M}\right\}$ e $N=4096 \ldots \ldots \ldots \ldots$ 
6.20 Estimativas pelo método de Abry-Veitch em função de $H_{\text {gen }}$ para $j_{\min } \in\left\{1, j_{\min }^{E Q M}\right\}$ e $N=65536$.

6.21 Estimativas pelo método de Abry-Veitch em função de $N$ para $H_{\text {gen }} \in\{0,6 ; 0,75 ; 0,9\}$ e $j_{\text {min }}=1$.

6.22 Estimativas pelo método de Abry-Veitch em função de $N$ para $H_{\text {gen }} \in\{0,6 ; 0,75 ; 0,9\}$ e $j_{\text {min }}=j_{\text {min }}^{E Q M}$

6.23 Espectro wavelet de quatro realizações distintas com $N=256$ e $H=0,6 \ldots \ldots \ldots \ldots \ldots \ldots \ldots \ldots$

6.24 Espectro wavelet de quatro realizações distintas com $N=256 \mathrm{e}$ $H=0,75$.

6.25 Espectro wavelet de quatro realizações distintas com $N=256$ e $H=0,9 \ldots \ldots \ldots \ldots \ldots \ldots \ldots \ldots$

6.26 Espectro wavelet de quatro realizações distintas com $N=4096$ e $H=0,6 \ldots \ldots \ldots \ldots \ldots \ldots \ldots \ldots$

6.27 Espectro wavelet de quatro realizações distintas com $N=4096$ e $H=0,75 \ldots \ldots \ldots \ldots \ldots \ldots \ldots$

6.28 Espectro wavelet de quatro realizações distintas com $N=4096$ e $H=0,9 \ldots \ldots \ldots \ldots \ldots \ldots \ldots \ldots$

6.29 Espectro wavelet de quatro realizações distintas com $N=65536$ e $H=0,6 \ldots \ldots \ldots \ldots \ldots \ldots \ldots \ldots$

6.30 Espectro wavelet de quatro realizações distintas com $N=65536$ e $H=0,75 \ldots \ldots \ldots \ldots \ldots \ldots \ldots$

6.31 Espectro wavelet de quatro realizações distintas com $N=65536$ e $H=0,9 \ldots \ldots \ldots \ldots \ldots \ldots \ldots$

6.32 Estimação de séries concatenadas (controle) - $H=0,6 ; f_{0}=0 \Longrightarrow$ $H=0,6 ; f_{0}=0 \ldots \ldots \ldots \ldots \ldots \ldots$

6.33 Estimação de séries concatenadas (controle) - $H=0,75 ; f_{0}=$ $0 \Longrightarrow H=0,75 ; f_{0}=0 \ldots \ldots \ldots \ldots$

6.34 Estimação de séries concatenadas (controle) - $H=0,9 ; f_{0}=0 \Longrightarrow$ $H=0,9 ; f_{0}=0 \ldots \ldots \ldots \ldots \ldots$

6.35 Estimação de séries concatenadas (controle) - $H=0,6 ; f_{0}=0 \Longrightarrow$ $H=0,9 ; f_{0}=0 \ldots \ldots \ldots \ldots \ldots$ 
6.36 Estimação de séries concatenadas (controle) - $H=0,9 ; f_{0}=0 \Longrightarrow$ $H=0,6 ; f_{0}=0 \ldots \ldots \ldots \ldots \ldots$

6.37 Estimação de séries concatenadas - $H=0,75 ; f_{0}=0 \Longrightarrow H=$ 0,$75 ; f_{0}=0,2 \ldots \ldots \ldots \ldots \ldots$

6.38 Estimação de séries concatenadas - $H=0,75 ; f_{0}=0 \Longrightarrow H=$ 0,$75 ; f_{0}=0,4 \ldots \ldots \ldots \ldots \ldots$

6.39 Estimação de séries concatenadas - $H=0,75 ; f_{0}=0 \Longrightarrow H=$ 0,$75 ; f_{0}=0,7$.

6.40 Estimação de séries concatenadas - $H=0,75 ; f_{0}=0,2 \Longrightarrow H=$ 0,$75 ; f_{0}=0,4$.

6.41 Estimação de séries concatenadas - $H=0,75 ; f_{0}=0,2 \Longrightarrow H=$ 0,$75 ; f_{0}=0,7$.

6.42 Espectro wavelet de séries concatenadas - $H=0,9 ; f_{0}=0 \Longrightarrow$ $H=0,6 ; f_{0}=0$.

6.43 Espectro wavelet de séries concatenadas - $H=0,75 ; f_{0}=0 \Longrightarrow$ $H=0,75 ; f_{0}=0,2 \ldots \ldots \ldots \ldots \ldots$

6.44 Espectro wavelet de séries concatenadas - $H=0,75 ; f_{0}=0,2 \Longrightarrow$ $H=0,75 ; f_{0}=0,7 \ldots \ldots \ldots \ldots$. . . . . . . . . . . . . .

6.45 Estudo da influência de $\log _{2} N$ na determinação de $j_{\min }^{E Q M}$ e de $\hat{H}$ - Séries originais sem SRD.

6.46 Estudo da influência de $\log _{2} N$ na determinação de $j_{\min }^{E Q M}$ e de $\hat{H}$ - Séries com SRD - $f_{0} \approx 0,2$.

6.47 Estudo da influência de $\log _{2} N$ na determinação de $j_{\min }^{E Q M}$ e de $\hat{H}$ - Séries com SRD - $f_{0} \approx 0,4$.

6.48 Estudo da influência de $\log _{2} N$ na determinação de $j_{\min }^{E Q M}$ e de $\hat{H}$ - Séries com SRD - $f_{0} \approx 0,7$.

6.49 Estudo da influência de $H_{g e n}$ na determinação de $j_{\min }^{E Q M}$ e de $\hat{H}-$ Séries com $N=2^{8}$ (parte 1) . . . . . . . . . . . . 118

6.50 Estudo da influência de $H_{g e n}$ na determinação de $j_{m i n}^{E Q M}$ e de $\hat{H}-$ Séries com $N=2^{8}$ (parte 2) . . . . . . . . . . . . . . . 119

6.51 Estudo da influência de $H_{g e n}$ na determinação de $j_{m i n}^{E Q M}$ e de $\hat{H}-$ Séries com $N=2^{10}$ (parte 1$)$. 
6.52 Estudo da influência de $H_{g e n}$ na determinação de $j_{\min }^{E Q M}$ e de $\hat{H}$ Séries com $N=2^{10}$ (parte 2) . . . . . . . . . . . . . 121

6.53 Estudo da influência de $H_{g e n}$ na determinação de $j_{\min }^{E Q M}$ e de $\hat{H}-$ Séries com $N=2^{12}$ (parte 1) . . . . . . . . . . . . . . . 122

6.54 Estudo da influência de $H_{g e n}$ na determinação de $j_{\min }^{E Q M}$ e de $\hat{H}$ Séries com $N=2^{12}$ (parte 2) . . . . . . . . . . . . . 123

6.55 Estudo da influência de $H_{g e n}$ na determinação de $j_{\min }^{E Q M}$ e de $\hat{H}$ Séries com $N=2^{14}$ (parte 1) . . . . . . . . . . . . . . 124

6.56 Estudo da influência de $H_{g e n}$ na determinação de $j_{\min }^{E Q M}$ e de $\hat{H}$ Séries com $N=2^{14}$ (parte 2) . . . . . . . . . . . . . 125

6.57 Estudo da influência de $H_{g e n}$ na determinação de $j_{\min }^{E Q M}$ e de $\hat{H}-$ Séries com $N=2^{16}$ (parte 1) . . . . . . . . . . . . 126

6.58 Estudo da influência de $H_{\text {gen }}$ na determinação de $j_{\min }^{E Q M}$ e de $\hat{H}-$ Séries com $N=2^{16}$ (parte 2) . . . . . . . . . . . . 127

7.1 Comparação do espectro wavelet obtido pela decomposição DWT e pela decomposição DWPT com filtro db1 - H=0,5. . . . . . . 131

7.2 Comparação do espectro wavelet obtido pela decomposição DWT e pela decomposição DWPT com filtro db1 - H=0,75 . . . . . . 132

7.3 Comparação do espectro wavelet obtido pela decomposição DWT e pela decomposição DWPT com filtro db1 - H=0,95 . . . . . . 133

7.4 Comparação do espectro wavelet obtido pela decomposição DWT e pela decomposição DWPT com filtro db3 $-H=0,5$. . . . . . 134

7.5 Comparação do espectro wavelet obtido pela decomposição DWT e pela decomposição DWPT com filtro db3 $-H=0,75$. . . . . . 135

7.6 Comparação do espectro wavelet obtido pela decomposição DWT e pela decomposição DWPT com filtro db3 $-H=0,95 \ldots$. . . . . 136

7.7 Comparação dos espectros wavelet para os casos sem e com componentes SRD - decomposição DWPT com filtro db1. . . . . . . . 137

7.8 Comparação dos espectros wavelet para os casos sem e com componentes SRD - decomposição DWPT com filtro db3. . . . . . . . 138

7.9 Comparação entre as árvore da decomposição DWT convencional e árvore da deconposição DWT "inversa". . . . . . . . . . . . . . . 139 
7.10 Comparação dos espectros wavelet para estimação do parâmetro

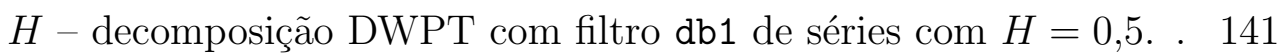

7.11 Comparação dos espectros wavelet para estimação do parâmetro $H$ - decomposição DWPT com filtro db1 de séries com $H=0,75.142$

7.12 Comparação dos espectros wavelet para estimação do parâmetro $H$ - decomposição DWPT com filtro db1 de séries com $H=0,95.143$

7.13 Comparação dos espectros wavelet para estimação do parâmetro $H$ - decomposição DWPT com filtro db3 de séries com $H=0,5.144$

7.14 Comparação dos espectros wavelet para estimação do parâmetro $H$ - decomposição DWPT com filtro db3 de séries com $H=0,75.145$

7.15 Comparação dos espectros wavelet para estimação do parâmetro $H$ - decomposição DWPT com filtro de séries com $H=0,95$. . . 146 


\section{Lista de Tabelas}

6.1 Resultados da Seção 6.1. . . . . . . . . . . . . . . . . . . . . 69

6.2 Resultados da Seção 6.2. . . . . . . . . . . . . . . . . . . . . 88

6.3 Resultados da Seção 6.3. . . . . . . . . . . . . . . . . . . 107

6.4 Compilação dos resultados teórico e empírico para estimação de H, segundo Abry, para modelos FGN e FARIMA. . . . . . . . . . 110

6.5 Resultados empíricos quantitativos da estimação de $H$ utilizandose modelos sem e com componentes SRD . . . . . . . . . . . . 111

6.6 Resultados da Seção 6.4. . . . . . . . . . . . . . . . . . . . . . 128

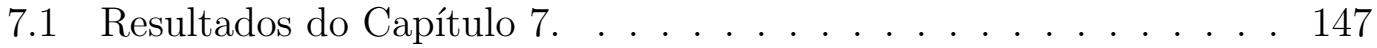




\section{Lista de Abreviaturas}

CAC Controle de Admissão de Conexões

CWT Continuous Wavelet Transform

DEP Densidade Espectral de Potência

DiffServ Differentiated Services

DWT Discrete Wavelet Transform

DWPT Discrete Wavelet Packet Transform

EQM Erro Quadrático Médio

FARIMA Fractional Autoregressive Integrated Moving Average

FGN Fractional Gaussian Noise

IntServ Integrated Services

IP Internet Protocol

IIR Infinite Impulse Response

LAN Local Area Network

LD Logscale Diagram

LRD Long-Range Dependence

MPLS Multiprotocol Label Switching

MRA Multiresolution Analysis

NGN Next Generation Networks

PDF Probability Density Function

QoS Quality of Service

QQ-plot Quantile-Quantile plot 
RB Ruído Branco

RBG Ruído Branco Gaussiano

R/S Range Over Standard Deviation

SLA Service Level Agreement

SRD Short-Range Dependence

UNC Universidade da Carolina do Norte

WAN Wide Area Network 


\section{Introdução}

Este Capítulo apresenta a motivação, os objetivos e as principais contribuições deste trabalho.

\subsection{Motivação}

Com o surgimento, nos últimos anos, das redes multisserviço, ou também chamadas Next Generation Networks (NGN), no âmbito das operadoras de telecomunicações, deu-se por iniciado o fenômeno de convergência de redes de dados e de telefonia preconizado há mais de uma década. Com tal integração entre redes, torna-se possível uma utilização mais racional de infraestrutura, através do compartilhamento de recursos e da integração do gerenciamento de tais recursos, implicando um aumento de eficiência e consequente redução de custos de operação de tais redes.

Entretanto, para que isso seja possível, é necessário que as redes Internet Protocol (IP) ofereçam garantias de Qualidade de Serviço, ou Quality of Service (QoS), não disponíveis em sua arquitetura original da década de 70, projetada para tráfego exclusivo de dados de aplicações elásticas a parâmetros como latência e banda. Alguns padrões e tecnologias têm sido propostos para que o desafio da garantia de QoS possa ser atingido em redes IP: Multiprotocol Label Switching (MPLS) [11, 12, 13], roteamento baseado em restrições (constrainedbased routing) [11], engenharia de tráfego, Serviços Integrados - Integrated Services (IntServ) - [14] e Serviços Diferenciados - Differentiated Services (DiffServ) $-[15,16,17,18]$.

Todas as tecnologias de QoS acima citadas implementam um ou mais mecanismos [19] que monitoram e/ou regulam o teletráfego para uma correta e eficiente operação das redes NGN. Na realidade, embora o objetivo final seja o controle do fluxo de tráfego de forma a se ter garantido um Service Level Agreement (SLA) pré-definido para uma aplicação ou um fluxo específicos, ou para a rede como um todo, para que tal controle seja eficaz é necessário algum tipo de monitora- 
mento do estado da rede para que sejam tomadas ações para garantia da QoS. É esse monitoramento que permitirá que seja feita uma inferência do estado da rede em instantes relativamente próximos ou distantes no futuro, de forma que mecanismos tais como o Controle de Admissão de Conexões (CAC) possam tomar decisões corretas e precisas e, assim, protejam a QoS percebido pelos usuários da rede.

Tal inferência do estado futuro de uma rede IP, ao contrário das redes de telefonia convencionais, está longe de ser trivial, uma vez que os tráfegos agregados de ambas as redes apresenta características estatísticas bastante distintas. Enquanto a rede de telefonia apresenta tráfego que pode ser modelado pelo processo de Poisson [20, 21], o tráfego das redes Local Area Network (LAN) [4] e Wide Area Network (WAN) [22] possui propriedades fractais tais como dependência de longa duração - Long-Range Dependence (LRD) - e impulsividade em diversas escalas de agregação temporal, que não são capturadas pelo processo de Poisson. Tal comportamento impulsivo ocorre porque o tráfego em tais redes apresenta uma distribuição marginal com cauda pesada (não Gaussiana) [23, 24, 25, 26, 27, 28].

As características fractais do tráfego agregado LRD podem ser caracterizadas de forma trivial pelo chamado parâmetro de Hurst (ou parâmetro $H$, a ser definido oportunamente), sendo tal parâmetro essencial para que possa ser feita a previsão do estado futuro do tráfego.

Estudos revelam, entretanto, que o tráfego IP pode não apresentar somente comportamento LRD, mas também componentes SRD ou ainda componentes periódicas $[29,30]$. Séries de teletráfego coletadas ${ }^{1}$ na Universidade da Carolina do Norte (UNC) exemplificam como o tráfego pode apresentar tanto comportamento puramente LRD como comportamentos distintos deste.

Conforme detalhado no artigo [30], a Figura 1.1 ilustra a série ${ }^{2}$ UNC-1, de duas horas de duração, com agregação de $1 \mathrm{~ms}$. A Figura 1.1a representa a série original com agregação de 1 ms, enquanto que a Figura 1.1b ilustra a mesma série com agregação de 1 s. A Figura 1.2c representa o espectro wavelet (conforme será descrito na Seção 4.3) de tal série, típico de séries com comportamento puramente LRD. A Figura 1.2, análoga à Figura 1.1, representa a série ${ }^{3}$ UNC-2. O gráfico da Figura 1.2c tem comportamento notoriamente distinto do da Figura 1.1c, apresentando um "pico" nas baixas escalas do espectro wavelet, indicando presença de outras componentes que não exclusivamente a componente LRD. Neste caso

\footnotetext{
${ }^{1}$ Séries temporais disponíveis em http://www-dirt.cs.unc.edu/ts/unc02_ts/

${ }^{2}$ Cf. arquivo 2002_Apr_13_Sat_1930.7260.sk1.1ms.B_P.ts.gz

${ }^{3}$ Cf. arquivo 2002_Apr_13_Sat_1300.7260.sk1.1ms.B_P.ts.gz
} 
em específico, são observadas componentes que indicam periodicidade em baixa frequência, conforme periodograma apresentado na Figura 1.3a e ampliação da visualização para baixas frequências na Figura 1.3b. A Figura 1.3c representa o mesmo periodograma em um gráfigo log-log.

A importância da detecção de componentes distintas da componente LRD, em séries de teletráfego, existe devido ao fato que tais componentes possuem a característica indesejada de interferir na estimação do parâmetro $H$ das séries de teletráfego [31], prejudicando inferências baseadas em tal estatística e, por conseguinte, o controle de QoS eventualmente realizado baseado nas inferências erroneamente realizadas.

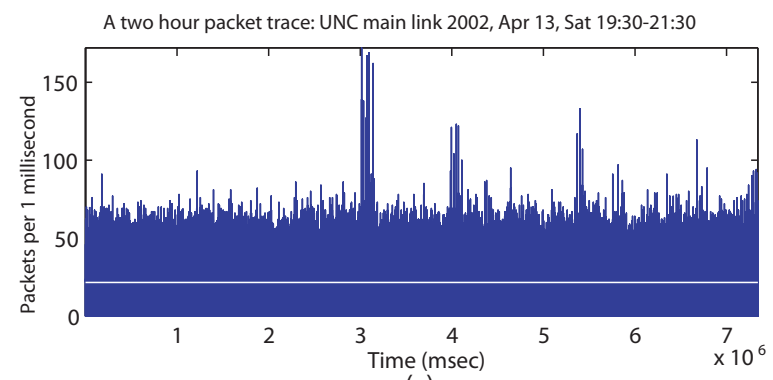

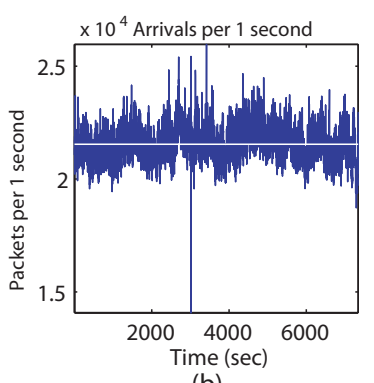

(b)

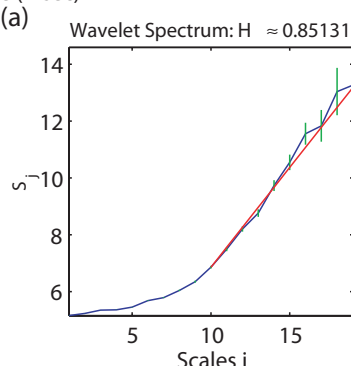

(c)

Figura 1.1: Observações temporais e do espectro wavelet da série UNC-1 [30].

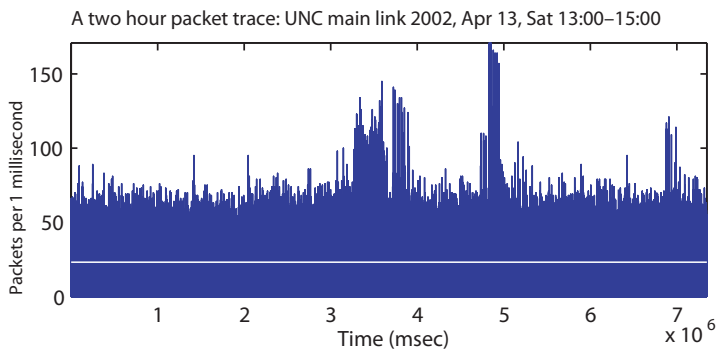

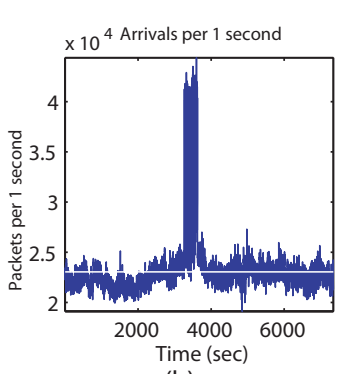

(b)

(a)

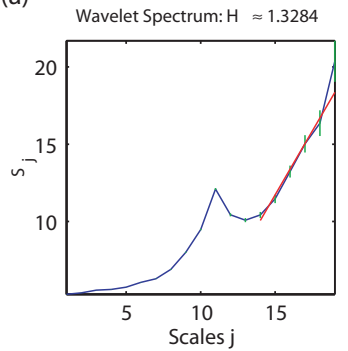

(c)

Figura 1.2: Observações temporais e do espectro wavelet da série UNC-2 [30]. 

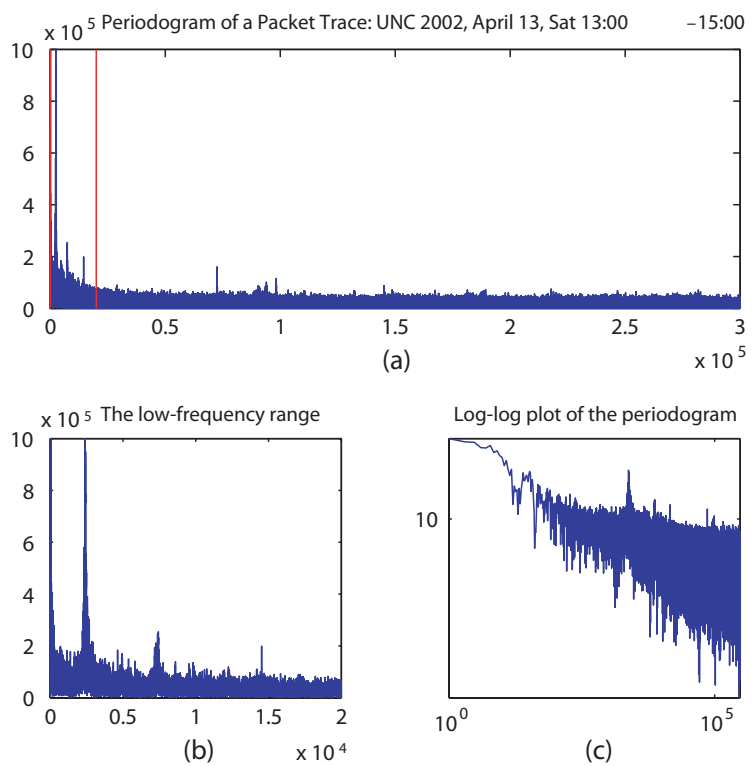

Figura 1.3: Periodograma da série UNC-2 [30].

\subsection{Objetivos e contribuições}

O objetivo do presente trabalho é apresentar o efeito potencialmente negativo da presença de componentes de dependência de curta duração na estimação do parâmetro de Hurst e, baseado em tais efeitos observados, propor alternativas para que possa ser realizada uma estimação em tempo real precisa das características de longa duração do tráfego, na presença de tais componentes.

Nesse sentido, buscou-se focar o estudo de um estimador específico, a saber, o estimador de Abry-Veitch [7, 32, 33]. Métodos semi-paramétricos de estimação do parâmetro $H$, tais como o método de Abry-Veitch, podem superar métodos paramétricos (tais como o de Whittle, descrito na Seção 4.2) na presença de SRD, uma vez que tais métodos não exigem o conhecimento a priori do modelo gerador da série temporal em estudo (o método de Whittle, por exemplo, assume que a série temporal em estudo apresenta comportamento exclusivamente LRD, podendo as estimativas por esse método estarem comprometidas no caso de uma componente SRD estar presente).

Desta maneira, o estimador de Abry-Veitch, baseado na estimação do parâmetro $H$ por meio do espectro wavelet da série sendo analisada, é dito como sendo robusto para estimação na presença de SRD - conforme estudos realizados utilizando-se o modelo Fractional Autoregressive Integrated Moving Average (FARIMA) em [10] - e é capaz de ser adaptado para estimação em tempo real (conforme descrito em $[34,35])$. Assim, trata-se de um forte canditato para estimador a ser utilizado, por exemplo, em um módulo de medição de QoS para controle em tempo real de redes NGN, na presença de teletráfego com características LRD e 
SRD, simultaneamente. Deve-se observar que não pertence ao escopo desta pesquisa a análise de séries de teletráfego que apresentem características tais como multifractalidade ou periodicidade.

Os estudos realizados concentraram-se na análise dos efeitos da variação dos parâmetros $j_{1}, j_{2}$ e $N$ do método de Abry-Veitch (conforme serão definidos na Seção 4.3) e na análise de tais efeitos na qualidade da estimação na presença de componente SRD na série sob análise. Embora o estudo da variação de $N$ já tenha sido realizado para efeitos de estimação em tempo real nos trabalhos [35] e [34], tais trabalhos não deram a devida atenção para séries com componentes $\mathrm{SRD}$, além de considerarem os parâmetros $j_{1}, j_{2}$ como fixos. Adicionalmente, a pesquisa realizada neste trabalho contemplou o estudo do algoritmo na presença de não estacionariedades com relação à componente SRD da série sendo analisada, obtendo resultados que permitem, por exemplo, identificar se uma aparente não estacionariedade ocorre devido a uma mudança do parâmetro $H$, ou devido a uma mudança nas componentes SRD da série. Os estudos, referentes a não estacionariedades, realizados até o momento com tal algoritmo [36, 37, 38] limitaram-se à análise da componente LRD ou do nível de tráfego. De forma complementar, o estudo do algoritmo de Abry-Veitch foi concluído através de um conjunto de simulações para comparação com os resultados obtidos em [10], simulações estas que possibilitaram a geração de dados detalhados referente ao comportamento das estimações em diferentes condições de tráfego e diferentes combinações dos parâmetros $j_{1}$ e $N$ do método de estimação.

Após os estudos realizados sobre o método de Abry-Veitch, foi observado que, em alguns casos, a variação dos parâmetros $j_{1}$ e $N$ não é suficiente para que a componente SRD da série seja inibida para efeito da estimação de $H$. Optou-se, assim, por dar início a um estudo para utilização do espectro wavelet obtido via Discrete Wavelet Packet Transform (DWPT) na estimação de $H$ - ao contrário da utilização do espectro wavelet obtido via Discrete Wavelet Transform (DWT) no caso do método de Abry-Veitch. Conforme será apresentado, a utilização da DWPT possui algumas vantagens sobre a DWT, uma vez que a primeira pode fornecer um espectro com mais flexibilidade na resolução tempo-frequência para estimação de $H$.

Por fim, os resultados obtidos nos estudos das séries via DWT e DWPT são revisitados e agrupados em uma proposta de novo mecanismo de estimação do parâmetro de Hurst, mecanismo este a ser utilizado na estimação em tempo real de séries com presença simultânea de componentes LRD e SRD. 


\subsection{Estrutura da dissertação}

O Capítulo 2 apresenta o conceito de fractal e formula os conceitos de LRD e autossimilaridade de segunda ordem. O Capítulo 3 introduz as wavelets no âmbito do estudo de séries de teletráfego, apresentando as principais transformadas utilizadas na análise das séries e estimação do parâmetro $H$. O Capítulo 4, que encerra a parte de fundamentação teórica deste trabalho, apresenta os principais conceitos da modelagem de tráfego fractal, tais como o modelo Fractional Gaussian Noise (FGN), e diversos métodos de estimação do parâmetro $H$, incluindo o método de Abry-Veitch.

A análise exploratória de estimação de $H$ é contextualizada no Capítulo 5. Os experimentos de estimação de $H$ via DWT e via DWPT são apresentados, respectivamente, nos Capítulos 6 e 7. Uma proposta de mecanismo de estimação de $H$ na presença de SRD é introduzida no Capítulo 8, com base nos resultados da análise exploratória.

Finalmente, o Capítulo 9 apresenta um resumo dos principais resultados obtidos, bem como sugestões de trabalhos futuros. 


\section{Parte I}

\section{Fundamentação teórica}




\section{A natureza fractal do teletráfego}

Este Capítulo introduz os conceitos de fractal, LRD e autossimilaridade de segunda ordem. Não faz parte do escopo desta dissertação uma abordagem detalhada dos conceitos de fractais e, muito menos, multifractais.

Ao leitor interessado em aprofundar-se nos conceitos da teoria de séries temporais, recomenda-se leitura das referências: [39], [40], [41], [42], [43], [7], [44], $[45]$ e $[46]$.

\subsection{Fractais}

O termo fractal (do latim fractus, fracionado, quebrado), proposto por Mandelbrot [40], refere-se aos objetos matemáticos que possuem uma estrutura rica em detalhes ao longo de muitas escalas de observação. Ao contrário das formas geométricas clássicas (círculos, quadrados, triângulo), que perdem suas estruturas quando ampliadas (enquanto um astronauta em órbita observa a Terra como sendo esférica, um indivíduo posicionado em sua superfície tem a impressão de que a mesma é plana), os fractais não possuem tal característica. Um exemplo matemático de fractal é o conjunto de Mandelbrot (o "boneco de pão-de-mel"), apresentado na Figura $2.1^{1}$. Deve-se observar que a Figura 2.1b, obtida por meio de um zoom de uma determinada região da Figura 2.1a, mostra que existe um sub-boneco de pão-de-mel embebido no boneco original.

A autossimilaridade é outra característica dos fractais: um objeto autossimilar contém réplicas menores de si mesmo em todas as escalas. Tal característica pode ser observada tanto em um sentido determinístico (como no conjunto de Mandelbrot, que é exatamente autossimilar), como em um sentido estatístico (em fractais "aleatórios"). Exemplos de tais fractais aleatórios são apresentados em inúmeros fenômenos que envolvam um extensa faixa de escalas de medição

\footnotetext{
${ }^{1}$ Gráficos obtidos utilizando-se o código MATLAB de A. Klimke [47].
} 


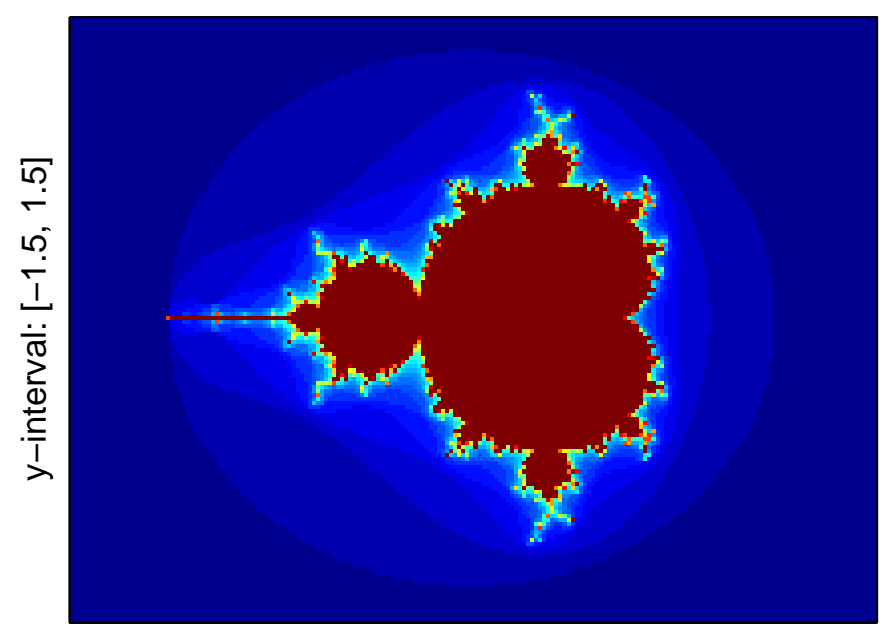

x-interval: $[-2.5,1.5]$

(a) Observação do boneco de pão-de-mel original

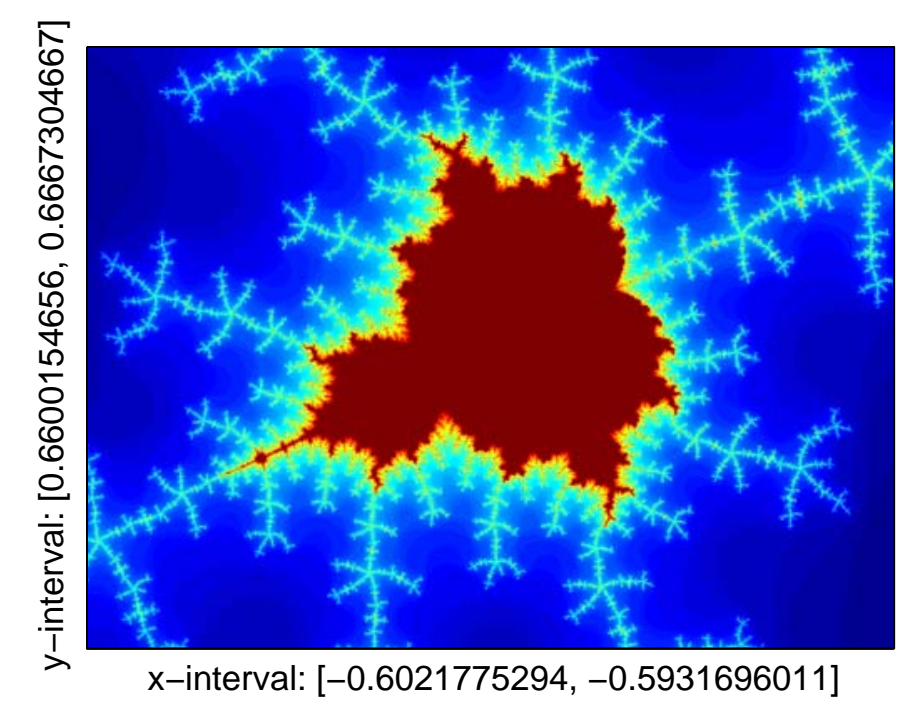

(b) Observação de um sub-boneco de pão-de-mel embebido no boneco de pão-de-mel original

Figura 2.1: Exemplo de visualização do conjunto de Mandelbrot (ou boneco de pão-de-mel). 


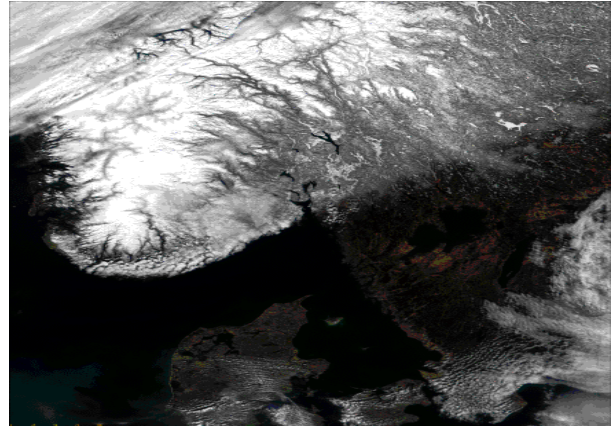

(a) Resolução: $1,4 \mathrm{~km}$

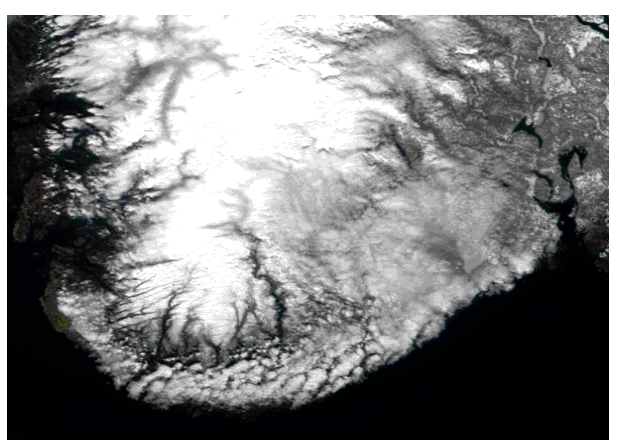

(b) Resolução: $500 \mathrm{~m}$

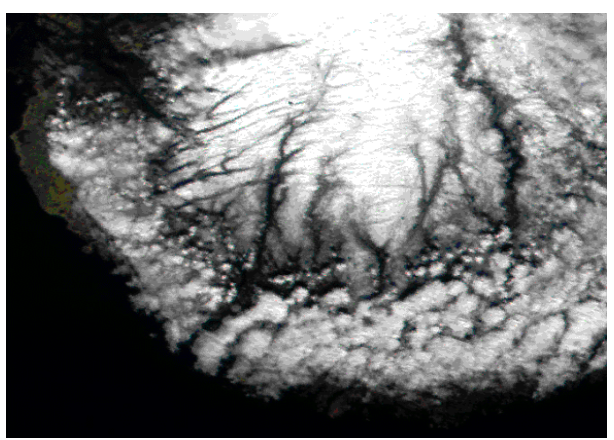

(c) Resoluação: $250 \mathrm{~m}$

Figura 2.2: Fotos que revelam a característica autossimilar do contorno do litoral da península Escandinava [48].

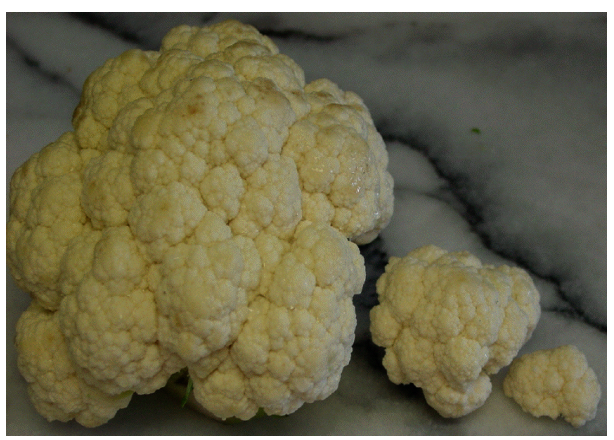

Figura 2.3: Foto que ilustra a característica autossimilar de uma couve-flor [48]. 
[39, 49] e não ocorrem em uma escala temporal ou espacial característica. Exemplos de tais fractais estatísticos são: séries temporais nas áreas de climatologia e hidrologia, séries de imagens de ressonância magnética funcional do cérebro humano, movimento turbulento de fluidos e séries de dados financeiros. A natureza também apresenta alguns exemplos de fractais [48], tais como a couve-flor (Figura 2.3) e o contorno de um litoral (Figura 2.2).

Um outro exemplo de séries que apresentam autossimilaridade é, justamente, o de séries de teletráfego de redes LAN [4] e WAN [22]. A Figura 2.4 ilustra o caso do tráfego de uma rede local da Universidade de Drexel [50]. A Figura apresenta a série original (com agregações de 10 ms, representada no topo) e diversas outras agregações no tempo, correspondentes à observação da mesma série em diversas outras escalas. A agregação de 100 ms é obtida agrupando-se os pontos da série original em bins de $100 \mathrm{~ms}$ cada, e então realizando a soma da contagem de bytes dos pontos da série correspondente a cada bin. As demais agregações são obtidas de forma análoga, sendo que de uma escala para a seguinte as agregações são feitas aumentando-se o tamanho do bin por um fator de 10. A evidência visual de que a série em estudo é autossimilar depreende-se da observação de que, para qualquer escala em questão, a mesma característica impulsiva (alternância de períodos de surtos e de suavidade) é observada em qualquer uma delas ${ }^{2}$.

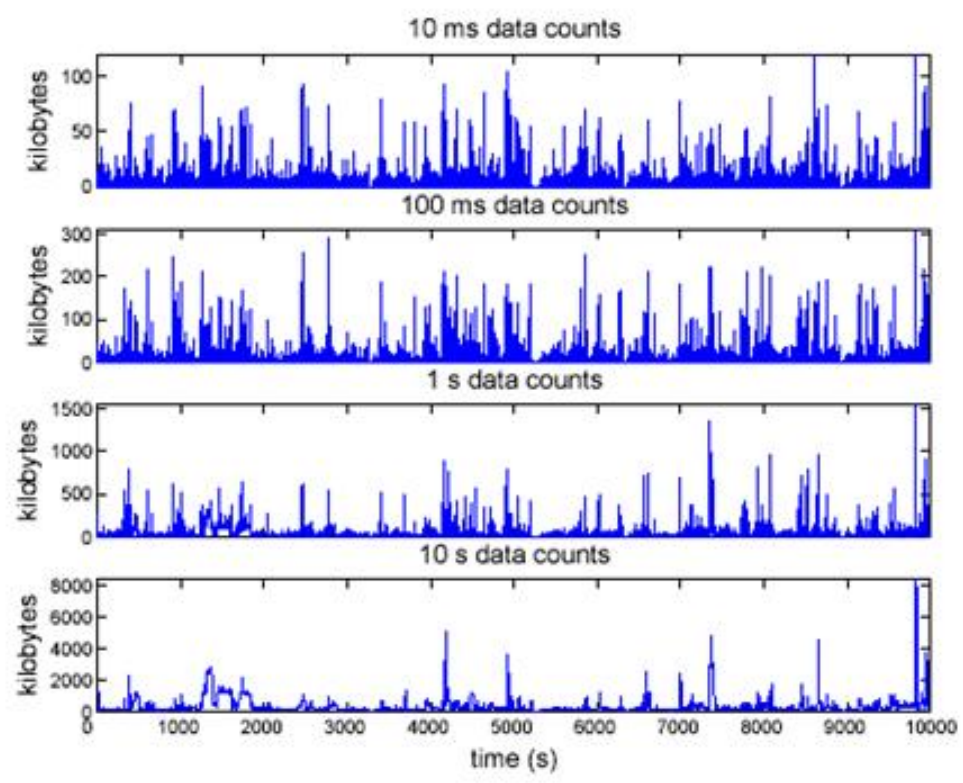

Figura 2.4: Tráfego Fast Ethernet coletado em um servidor da Universidade de Drexel, apresentado em 4 diferentes níveis de agregação [50].

De forma comparativa, a Figura 2.5 ilustra o comportamento de uma série que não apresenta comportamento autossimilar, através da comparação entre uma sé-

\footnotetext{
${ }^{2} \mathrm{~A}$ literatura refere-se a tal propriedade de invariância com relação à mudança de escala temporal como "scaling invariance" ou "scaling behavior"[42, 29].
} 
rie de tráfego Ethernet real e o tráfego simulado por meio do modelo de Poisson [4]. Os gráficos à esquerda, representam as diversas agregações do tráfego real, enquanto os gráficos na coluna do meio referem-se às agregações de um tráfego simulado segundo o modelo de Poisson. As escalas de observação originais (menos agregadas) de ambos os tipos de tráfego são apresentadas na parte inferior. Entretanto, para as observações de séries mais agregadas, apresentadas no topo do gráfico, observa-se que, enquanto o tráfego Ethernet mantém suas características impulsivas (conforme já apresentado), o tráfego Poisson torna-se bastante suavizado, não mantendo suas características estatísticas ao longo das diversas escalas de agregação ${ }^{3}$. As séries na coluna da direita da Figura representam o tráfego sintetizado por meio de um modelo autossimilar que, conforme observado, mantém em diversas escalas de tempo a característica de alternância de períodos de surtos e de suavidade.
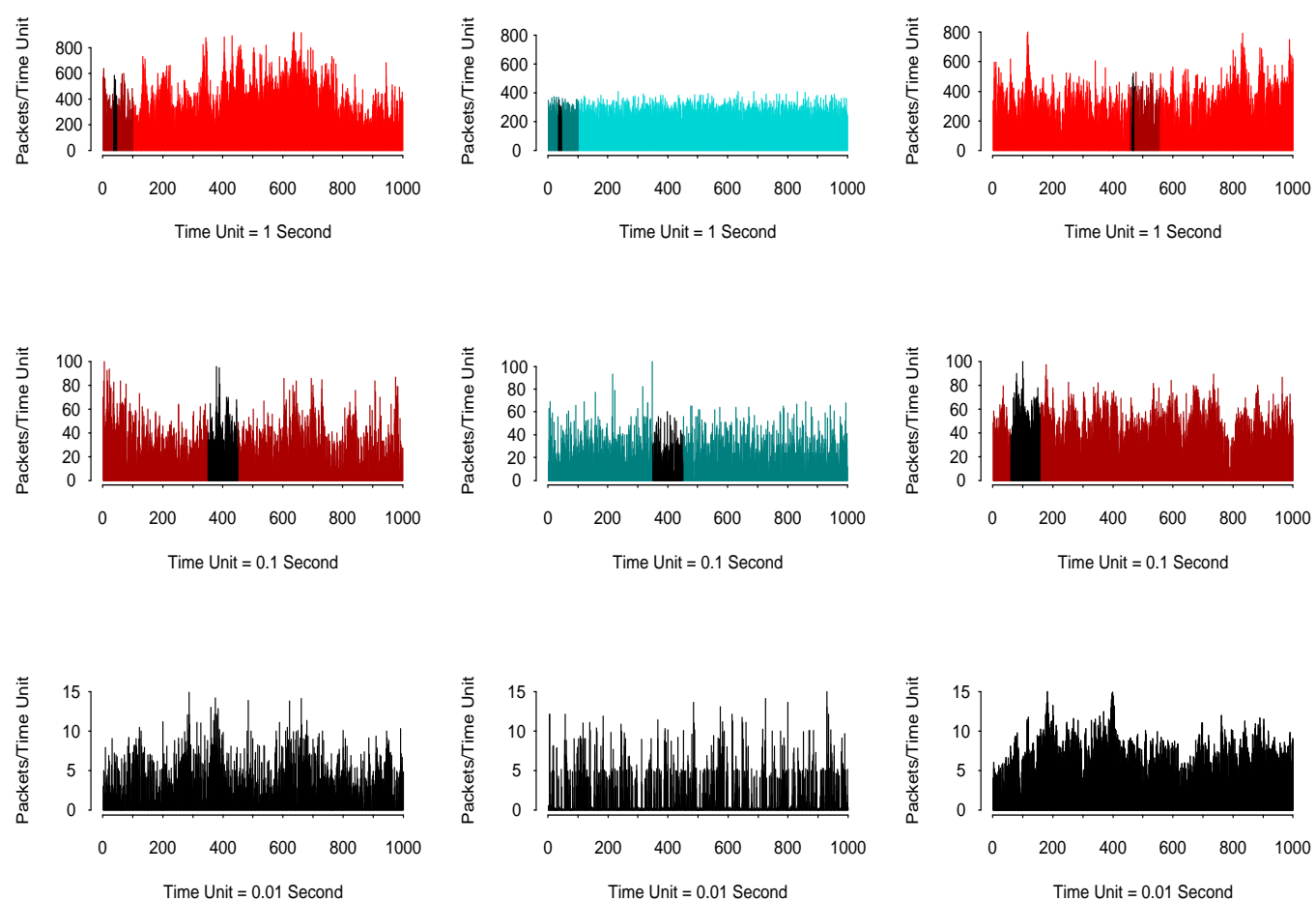

Figura 2.5: Comparação das características impulsivas, em diversas escalas de agregação, do tráfego Ethernet real e sintetizado [4].

\subsection{Dependência de longa duração}

O seguinte desenvolvimento matemático segue a linha apresentada em [3]. Considere um processo aleatório estacionário $\boldsymbol{x}_{t}, t \in \mathbb{Z}$, com média $\mu_{\boldsymbol{x}}$ e variância $\sigma_{\boldsymbol{x}}^{2}$.

\footnotetext{
${ }^{3}$ Agregações sucessivas de uma série temporal de Poisson tendem a produzir séries do tipo Ruído Branco (RB), conforme observado na Figura 2.5.
} 
Sejam $x_{1}, x_{2}, \ldots, x_{N}$ observações de uma realização $x_{t}$. A seguinte definição de dependência de longa duração é apresentada no domínio da frequência.

Definição 2.1 (Dependência de Longa Duração - domínio da frequência). $\boldsymbol{x}_{t}$ é um processo com dependência de longa duração ou memória longa se existem constantes $\alpha$ e $C_{P}$, satisfazendo $0<\alpha<1$ e $C_{P}>0$, tais que $[39,49,51]$

$$
\lim _{f \rightarrow 0} \frac{P_{x}(f)}{C_{P}|f|^{-\alpha}}=1
$$

em que $P_{x}(f)$ denota a Densidade Espectral de Potência (DEP) de $\boldsymbol{x}_{t}$ e $f$ representa a frequência normalizada $(-1 / 2 \leq f \leq 1 / 2)$, em ciclos/amostra.

Uma definição alternativa de dependência de longa duração pode ser dada no domínio do tempo, em termos de sua autocorrelação $R_{x}(\tau)$.

Definição 2.2 (Dependência de Longa Duração - domínio do tempo). O processo $\boldsymbol{x}_{t}$ é do tipo $1 / f^{\alpha}$ se a sua autocorrelação $R_{x}(\tau)$, para valores suficientemente grandes do $\operatorname{lag} \tau$, decresce segundo uma função potência:

$$
\lim _{\tau \rightarrow \infty} \frac{R_{x}(\tau)}{C_{R} \tau^{-(1-\alpha)}}=1
$$

em que $C_{R}>0$.

A caracterização da memória longa pelo parâmetro $H$ de $\mathrm{Hurst}^{4}$ [52] é, por razões históricas, mais comum do que pelo parâmetro $\alpha$ :

$$
H=\frac{\alpha+1}{2}, \quad 1 / 2<H<1
$$

Uma série temporal é LRD (autossimilar ${ }^{5}$ ) quando $1 / 2<H<1$ e tem memória curta ou dependência de curta duração (SRD) para $0<H \leq 1 / 2$ [39]. Quanto mais próximo $H$ estiver de 1, maior será o grau de persistência da série. Quanto maior o valor de $H$, maior é o grau de memória longa do processo.

Observa-se das Definições 2.1 e 2.2 que:

1. a DEP de processos LRD tende a infinito na frequência zero (singularidade do tipo $1 / f^{\alpha}$ na origem do espectro);

2. o formato da DEP em frequências afastadas da origem não é especificado, uma vez que a definição acima é assintótica;

\footnotetext{
${ }^{4}$ Hurst, um hidrologista, observou que a série histórica do nível mínimo anual do rio Nilo possui memória longa.

${ }^{5} \mathrm{No}$ contexto desta pesquisa, os termos memória longa e autossimilaridade são sinônimos. Conforme será apresentado na Seção 2.3, embora, a rigor, autossimilaridade e dependência de longa duração sejam conceitos distintos, processos estocásticos autossimilares de segunda ordem são LRD e vice-versa.
} 
3. o decaimento para zero da autocorrelação $R_{x}(\tau)$ de um processo LRD é extremamente lento, do tipo hiperbólico;

4. considerando-se a singularidade na origem do espectro do processo LRD, depreende-se que $\sum_{\tau=-\infty}^{\infty} R_{x}(\tau)=\infty$ (para $1 / 2<H<1$ ), indicando que as autocorrelações decaem para zero tão lentamente que não são somáveis, o que implica que a dependência estatística entre eventos distantes diminui muito lentamente com o aumento do $\operatorname{lag} \tau$;

5. para valores suficientemente grandes do $l a g$, a razão entre autocorrelações não se altera de modo apreciável, comportamento este oposto ao apresentado por processos que possuam dependência de curta duração.

A relevância em caracterizar corretamente uma série $x_{t}$ como sendo LRD vem do fato de que a estimação de parâmetros como $\bar{x}$ é mais difícil do que quando as observações são não correlacionadas. Isso porque a variância de $\bar{x}$ decresce com o tamanho $N$ da amostra mais lentamente do que no caso tradicional (variáveis independentes ou não correlacionadas), conforme a equação [39, pág.6]

$$
\sigma_{\bar{x}}^{2} \approx \sigma_{x}^{2} c\left(\rho_{x}\right) N^{\alpha-1}
$$

em que $c\left(\rho_{x}\right)$ é definido por

$$
\lim _{N \rightarrow \infty} N^{-(1+\alpha)} \sum_{i \neq j} \rho_{x}(i, j)
$$

Observando-se as Equações 2.4 e 2.5 para o caso em que $\alpha=0$, tem-se a variância para o caso de séries puramente SRD. Já para o caso limite em que $\alpha \rightarrow 1$, observa-se que a variância de $\bar{x}$ tende a infinito, inviabilizando tal estimação.

Finalmente, convém observar as propriedades estatísticas de um processo LRD em relação aos seus respectivos processos agregados ${ }^{6}$. O processo agregado de $\boldsymbol{x}_{t}$ de grau $M$, denotado por $X_{t}^{(M)}$, corresponde a uma média móvel de blocos não sobrepostos de $\boldsymbol{x}_{t}$ de tamanho $M$, ou seja,

$$
X_{i}^{(M)}=\frac{1}{M} \sum_{t=M(i-1)+1}^{M i} \boldsymbol{x}_{t} .
$$

A seguinte propriedade é válida para um processo $\boldsymbol{x}_{t}$ com memória longa [28]:

$$
\lim _{M \rightarrow \infty} \frac{\operatorname{Var} X_{t}^{(M)}}{M^{(2 H-2)}}=c
$$

em que $c$ é uma constante.

\footnotetext{
${ }^{6}$ A agregação equivale a uma operação de mudança de escala temporal.
} 
A Equação (2.7) afirma que o grau de suavidade da sequência de processos agregados $\left\{X_{t}^{(M)}\right\}_{M=2,3, \ldots}$, associados a um processo LRD $\boldsymbol{x}_{t}$, aumenta com o aumento de $M$ muito mais lentamente do que no caso de processos $\mathrm{SRD}$, em que a variância do processo agregado decai proporcionalmente a $M^{-1}$ para $M \rightarrow \infty$. Sendo assim, depreende-se que o processo agregado é estatisticamente similar ao processo original, no sentido de que um número finito de agregações sucessivas não destrói o caráter impulsivo do processo original (ao contrário do observado para processos SRD como o processo de Poisson, conforme visto anteriormente na Figura 2.5. Portanto, (2.7) sugere que as propriedades de dependência de longa duração e autossimilaridade estejam intimamente relacionadas, conforme definido a seguir na Seção 2.3.

\subsection{Autossimilaridade}

Definição 2.3 (Processo $\boldsymbol{H}$-ss). Um processo estocástico $\left\{\boldsymbol{y}_{t}\right\}_{t \in \mathbb{R}}$ é autossimilar com parâmetro $0<H<1$, ou seja, é $H$-ss (self-similar with parameter $H$ ) se, para qualquer $a>0$,

$$
\{\boldsymbol{y}(t)\} \stackrel{d}{=}\left\{a^{-H} \boldsymbol{y}(a t)\right\}
$$

em que $\stackrel{d}{=}$ denota igualdade entre as distribuições finito-dimensionais [51].

Um processo $H$-ss é LRD se $1 / 2<H<1$. O processo Movimento Browniano (de tempo contínuo) [53], também conhecido como processo de Wiener, satisfaz a definição 2.3, sendo autossimilar com $H=1 / 2$, mas não é LRD.

Definição 2.4 (Processo $\boldsymbol{H}$-sssi). Se o processo $\boldsymbol{x}_{t}=\Delta \boldsymbol{y}_{t}$, denominado processo de incrementos de $\boldsymbol{y}_{t}$ ou primeira diferença de $\boldsymbol{y}_{t}$, for estacionário, então $\boldsymbol{y}_{t}$ é denominado $H$-sssi ( $H$ self-similar with stationary increments - autossimilar com incrementos estacionários).

Definição 2.5 (Autossimilaridade Exata de Segunda Ordem). Seja o processo estacionário de tempo discreto $\boldsymbol{x}_{t}=\boldsymbol{y}_{t}-\boldsymbol{y}_{t-1}$. $\boldsymbol{x}_{t}$ é um processo exatamente autossimilar de segunda ordem com parâmetro de Hurst $H(1 / 2<H<1)$ se a sua autocovariância existe e é dada por [39]

$$
C_{x}(\tau)=\frac{\sigma_{x}^{2}}{2}\left[|\tau+1|^{2 H}-2|\tau|^{2 H}+|\tau-1|^{2 H}\right], \quad \tau=\ldots,-1,0,1, \ldots
$$

Pode-se demonstrar que a autocovariância dada pela Equação (2.9) satisfaz [39]

$$
\lim _{\tau \rightarrow \infty} \frac{C_{x}(\tau)}{\sigma_{x}^{2} \tau^{2 H-2} H(2 H-1)}=1
$$


ou seja, $C_{x}(\tau)$ tem um decaimento hiperbólico. Portanto, autossimilaridade de segunda ordem implica LRD quando $1 / 2<H<1$.

Considere o processo agregado $X_{t}^{(M)}$ de um processo exatamente autossimilar de segunda ordem $\boldsymbol{x}_{t}$, ao nível de agregação $M$. Demonstra-se que

$$
C_{\boldsymbol{x}}^{(M)}(\tau)=C_{\boldsymbol{x}}(\tau), \quad M=2,3, \ldots
$$

A Equação (2.11) afirma que as estatísticas de segunda ordem do processo original não mudam com a mudança de escala, o que justifica o termo "exatamente autossimilar de segunda ordem".

Definição 2.6 (Autossimilaridade Assintótica de Segunda Ordem). Um processo $\boldsymbol{x}_{t}$ é assintoticamente autossimilar de segunda ordem com parâmetro de Hurst $H$ $(1 / 2<H<1)$ se a sua autocovariância e a autocovariância do seu processo agregado estão relacionadas por [28]

$$
\lim _{M \rightarrow \infty} C_{x}^{(M)}(\tau)=C_{x}(\tau)
$$

É possível demonstrar [54] que (2.7) implica (2.12). Portanto, um processo LRD também é assintoticamente autossimilar de segunda ordem. 


\section{$3 \quad$ Wavelets}

Este Capítulo apresenta conceitos da teoria das wavelet, bem como as principais transformadas wavelets. Os principais conceitos wavelet são apresentados como ponto de partida para o estudo da DWT e DWPT. Enquanto a transformada DWT e a análise multirresolução são a base do método de estimação de $H$ de Abry-Veitch, a transformada DWPT será objeto de estudo para uma alternativa de estimação ao método de Abry-Veitch. Por fim, as principais características dos coeficientes wavelet de processos autossimilares e LRD são apresentadas.

Ao leitor interessado em aprofundar-se nos conceitos da teoria de wavelets e a sua aplicação no estudo de séries temporais, recomenda-se leitura das referências: [55], [56], [57], [58], [59], [60], [61], [62], [49] e [63].

\subsection{Introdução}

O seguinte desenvolvimento matemático segue a linha apresentada em [10]. Uma wavelet é uma função $\psi(t), t \in \mathbb{R}$ tal que

$$
\int_{\mathbb{R}} \psi(t) d t=0
$$

que também satisfaz algumas condições de integrabilidade. Em geral, é requisitado também que a wavelet seja limitada, centrada na origem, e tenha suporte que seja finito ou que decresça muito rapidamente conforme $|t| \rightarrow \infty$ (e, consequentemente, conforme $|\nu| \rightarrow \infty$, onde $\nu$ denota a frequência em ciclos/segundo $[\mathrm{Hz}])$. O princípio de incerteza de Gabor-Heisenberg [64, pág. 52] restringe as dispersões no tempo e frequência e, idealmente, deseja-se que as dispersões conjuntas no tempo e em frequência de $\psi$ sejam as mais próximas possível do limite teórico inferior. De forma intuitiva, o termo "wavelet" (ou "pequena onda", em tradução livre) é consequência de tais requisitos: uma natureza oscilante (conforme imposto por (3.1)), com pequeno suporte no tempo.

Uma wavelet $\psi$ é dita ter $N$ momentos ou cumulantes nulos (vanishing mo- 
ments) se

$$
\int_{\mathbb{R}} t^{k} \psi(t) d t=0, k=0,1, \ldots, N-1 .
$$

Em vista da condição fundamental (3.1), o inteiro $N$ é sempre ao menos igual a 1 . Usualmente, um aumento no número de cumulantes nulos resulta em um aumento do suporte no tempo, porém traz mais regularidade (suavidade, continuidade, derivabilidade) e uma melhor concentração do conteúdo espectral no entorno de uma dada frequência $\nu_{0}$.

Exemplos típicos de wavelets incluem as derivadas da PDF Gaussiana:

$$
\psi(t)=\left(d^{n} / d t^{n}\right)\left((2 \pi)^{1 / 2} e^{-t^{2} / 2}\right)
$$

a chamada wavelet de Haar:

$$
\psi(t)= \begin{cases}1 & \text { se } 0 \leq t<1 / 2 \\ -1 & \text { se } 1 / 2 \leq t<1 \\ 0 & \text { caso contrário }\end{cases}
$$

e as wavelets de Daubechies, construídas a partir da análise multirresolução (conforme Seção 3.2.1).

A wavelet "chapéu mexicano" (segunda derivada da PDF Gaussiana), por exemplo, tem dois cumulantes nulos e suporte em tempo e frequência que, estritamente falando, são infinitos, mas que são muito bem concentrados (vide Figura 3.1). As wavelets de Daubechies contituem uma família de wavelets que é indexada pelo seu número de cumulante nulos, e que dá origem a uma base wavelet ortonormal. A wavelet de Haar é um elemento especial da família Daubechies que tem um único cumulante nulo.

\subsection{Transformada DWT}

As funções

$$
\psi_{j, k}(t)=\frac{1}{2^{j / 2}} \psi\left(2^{-j} t-k\right)=2^{-j / 2} \psi\left(2^{-j}\left(t-2^{j} k\right)\right), j \in \mathbb{Z}, k \in \mathbb{Z},
$$

são "dilações" e "translações" de $\psi$. A função $\psi\left(2^{-1} t\right)$, por exemplo, é a dilação de $\psi$ por um fator de 2 , a função $\psi(t-k)$ é uma translação de $\psi$ para a direita em $k$ unidades e, considerando que $\psi$ tem suporte em $[0,1]$, então, por exemplo, $\psi\left(2^{-1} t\right)-3=\psi\left(2^{-1}(t-6)\right)$ tem suporte no intervalo [6,8]. Os fatores $2^{j}$ e $j$ são denominados, respectivamente, escala e oitava. Valores positivos de $j$ cor-

\footnotetext{
${ }^{1}$ Dilations, em inglês. O termo "dilação" tem o significado de dilatação.
} 


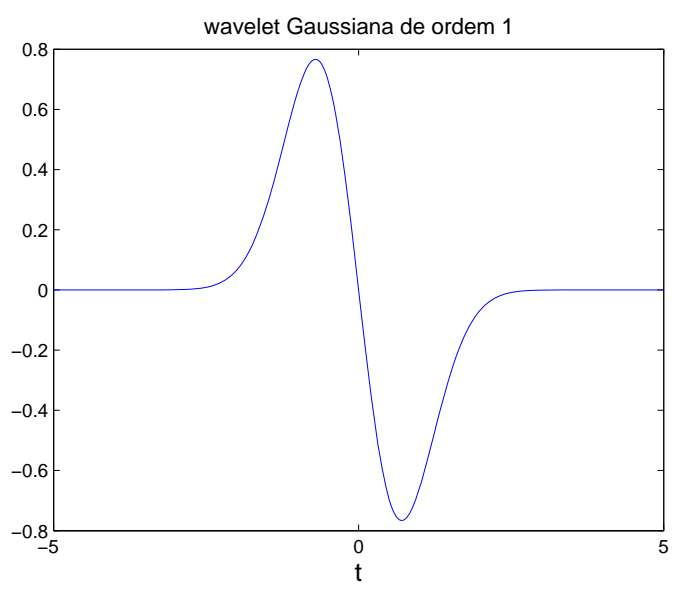

(a) A wavelet Gaussiana (relacionada à primeira derivada da PDF Gaussiana)

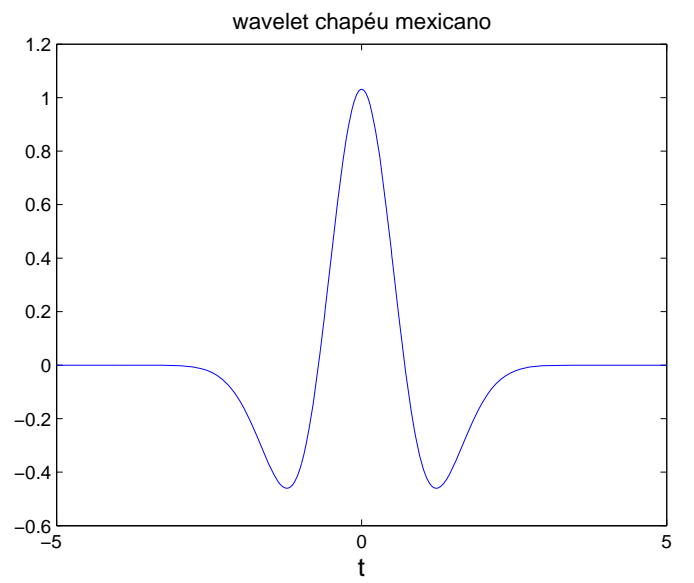

(b) A wavelet "chapéu mexicano" (relacionada à segunda derivada da PDF Gaussiana)

Figura 3.1: Exemplo de wavelets obtidas das derivadas da PDF Gaussiana [3]. 
respondem a dilações e valores negativos correspondem a contrações. O fator de normalização $2^{j / 2}$ garante que, para todo $j \in \mathbb{Z}$ e $k \in \mathbb{Z}$,

$$
\int_{\mathbb{R}} \psi_{j, k}^{2}(t) d t=\int_{\mathbb{R}} \psi^{2}(t) d t
$$

ou seja, ele permite que a norma $L^{2}(\mathbb{R})$ seja preservada.

Usando as funções $\psi_{j, k}, j, k \in \mathbb{Z}$ em (3.5) como um conjunto de filtros, podese definir a Transformada Wavelet Discreta (DWT) de uma função (ou de uma realização de um processo estocástico) $X(t), t \in \mathbb{R}$ como

$$
d_{j, k}=\int_{\mathbb{R}} X(t) \psi_{j, k}(t) d t \quad j, k \in \mathbb{Z}
$$

Os coeficientes $d_{j, k}$ são os chamados coeficientes wavelet, ou ainda coeficientes de detalhe, uma vez que eles codificam uma informação diferencial entre escalas adjacentes centradas en torno da escala $2^{j}$ e do instante de tempo $2^{j} k$. Essa característica de 'detalhe' dos detalhes é descrita na Seção 3.2.1, e é derivada da caracterísica passa banda de $\psi$ (cf. Equação (3.1)). À escala $2^{j}$ pode ser associada uma frequência $2^{-j} \nu_{0}$, onde $\nu_{0}$ é a frequência central característica da wavelet $\psi$.

Apesar de $X$ ser uma função do parâmetro de tempo contínuo $t$ e de envolver uma integral, convém ressaltar que o adjetivo "discreta" da Transformada Wavelet Discreta refere-se ao fato de que os índices $j, k$ assumem valores discretos, em contraste com a Transformada Wavelet Contínua - Continuous Wavelet Transform (CWT) - em que tais índices assumem valores reais [10].

\subsubsection{Análise Multirresolução}

A Teoria da Análise Multirresolução [58] - Multiresolution Analysis (MRA) consiste no estudo de uma série examinando suas "aproximações" de forma cada vez menos refinada, cancelando mais e mais as altas frequências, ou "detalhes", da série em questão. Os principais pontos de tal teoria podem ser assim resumidos (cf. [10]):

- a wavelet $\psi$ (a wavelet mãe) é definida através da função de escala $\phi$;

- tanto $\phi$ como $\psi$ satisfazem, respectivamente, à equação de dilação e à equa- 
ção da wavelet:

$$
\begin{aligned}
\phi(t / 2) & =\sqrt{2} \sum_{n} u_{n} \phi(t-n) \\
\psi(t / 2) & =\sqrt{2} \sum_{n} v_{n} \phi(t-n) .
\end{aligned}
$$

Por exemplo, para a wavelet de Haar, a função de escala $\phi(t)$ é a função característica do intervalo $[0,1], u_{0}=u_{1}=1 / \sqrt{2}$ e $u_{n}=0$ caso contrário, $-v_{1}=v_{0}=1 / \sqrt{2}$ e $v_{n}=0$ caso contrário;

- as sequências $u$ e $v$ são geradoras da análise multirresolução. Em particular, elas geram $\psi$ e $\phi$ e controlam suas propriedades, notavelmente o número de cumulantes nulos, regularidade e resolução conjunta tempo-frequência;

- adicionalmente aos coeficientes wavelet $d_{j, k}$ em (3.7), são também definidos os coeficientes de aproximação $a_{j, k}$ :

$$
a_{j, k}=\int_{\mathbb{R}} X(t) \phi_{j, k}(t) d t \quad j, k \in \mathbb{Z},
$$

onde $\phi_{j, k}(t)=2^{-j / 2} \phi\left(2^{-j} t-k\right)$. A relação (3.10) é análoga à relação (3.7), de forma que $\psi$ é substituido por $\phi$.

A maior consequência de (3.8) é o fato de que ambos os coeficientes de aproximação e wavelet $a_{j, k}$ e $d_{j, k}$ na escala $j$ podem ser computados de uma sequência de aproximação $\left\{a_{j-1, k}, k \in \mathbb{Z}\right\}$ em uma resolução mais refinada $j-1$ :

$$
\begin{aligned}
a_{j, k} & =\int_{\mathbb{R}} X(t) 2^{-j / 2} \phi\left(2^{-j} t-k\right) d t \\
& =\int_{\mathbb{R}} X(t) 2^{-j / 2} \sqrt{2} \sum_{n} u_{n} \phi\left(2\left(2^{-j} t-k\right)-n\right) d t \\
& =\sum_{n} u_{n} \int_{\mathbb{R}} X(t) 2^{(-j+1) / 2} \phi\left(2^{-j+1} t-2 k-n\right) d t \\
& =\sum_{n} u_{n} a_{j-1,2 k+n} \\
& =\sum_{n} u_{n}^{\vee} a_{j-1,2 k-n} \\
& =\left(u^{\vee} * a_{j-1, \cdot}\right)(2 k)
\end{aligned}
$$

e, de forma semelhante,

$$
d_{j, k}=\left(v^{\vee} * a_{j-1, \cdot}\right)(2 k)
$$

onde $*$ representa a operação de convolução em tempo discreto, ou seja $(x$ * $y$.) $(k)=\sum_{n} x(n) y(k-n), u_{n}^{\vee}=u_{-n}$ e, de forma semelhante, $v_{n}^{\vee}=v_{-n}$. As duas relações (3.11) e (3.12) podem ser reescritas utilizando-se o operador de decimação $\downarrow_{2}\left(y=\downarrow_{2} x\right.$ significa que $y_{k}=x_{2 k}$, ou seja, que uma em cada duas 
amostras de $x$ é descartada):

$$
\begin{aligned}
a_{j, k} & =\left[\downarrow_{2}\left(u^{\vee} * a_{j-1, .}\right)\right](k) \\
d_{j, k} & =\left[\downarrow_{2}\left(v^{\vee} * a_{j-1, .}\right)\right](k) .
\end{aligned}
$$

Embora estas equações aparentem ser o resultado de equações triviais, tais equações implicam na seguinte interpretação fundamental, central à intuição da análise multirresolução: uma aproximação $a_{j, k}$ dos dados na oitava $j$ é dividida em uma aproximação mais grosseira $a_{j+1, k}$ e um detalhe $d_{j+1, k}$. Este procedimento é repetido de forma recursiva a cada aproximação $a_{j, k}$ de forma que a sequência inicial $\left\{a_{0, k}, k \in \mathbb{Z}\right\}$ é reescrita como uma coleção de sequências de detalhes $\left\{\left\{d_{j, k}, k \in \mathbb{Z}\right\}, j=1, \ldots, J\right\}$ e uma aproximação final mais grosseira $\left\{a_{J, k}, k \in \mathbb{Z}\right\}$.

Os seguintes comentários podem ser feitos com relação às Equações (3.13) (cf. [10]):

- O cálculo dos coeficientes $d_{j, k}$ pode se realizado utilizando um algoritmo piramidal baseado em um banco de filtros de tempo-discreto, esboçado na Figura 3.2. Seu bloco elementar consiste de dois filtros de tempo discreto com respostas impulsivas $u^{\vee}$ e $v^{\vee}$, e operadores de decimação. Uma vez que os coeficientes $d_{j, k}$ não são computados do processo original de tempo contínuo $X$, mas de uma aproximação prévia de tempo-discreto $a_{j-1, k}$, o algoritmo resultante tem, particularmente, um baixo custo computacional, da ordem de $O(n)$ para computar $n$ coeficientes wavelet [49].

- O único estágio em que está envolvido cálculo de tempo contínuo é a computação da sequência inicial $a_{0, k}$ que requer um produto interno de tempo contínuo, a integral

$$
a_{0, k}=\int_{\mathbb{R}} X(t) \phi(t-k) d t .
$$

O restante do cálculo envolve apenas convoluções discretas. A derivação desta sequência inicial é conhecida como passo de inicialização e pode ser difícil por causa de sua natureza contínua. Um exemplo clássico que surge na prática é quando somente uma amostragem da realização de tempo contínuo está disponível, ao invés da realização inteira.

- As sequências $u^{\vee}$ e $v^{\vee}$ têm um papel importante nos filtros passa-baixa e passa-alta, respectivamente. A sequência $u^{\vee}$. dá origem a uma aproximação mais grosseira (permitindo a passagem das frequências baixas), enquanto 
que a sequência $v^{\vee}$ tende a preservar os detalhes (altas frequências).

De uma perspectiva mais abstrata, estas sequências permitem a decomposição do espaço $L^{2}(\mathbb{R})$ em subespaços fechados,

$$
L^{2}(\mathbb{R}) \supset \cdots \supset V_{-1} \supset V_{0} \supset V_{1} \supset \cdots
$$

Para um dado $j$, os $\phi_{j, k}, k \in \mathbb{Z}$, projetam o sinal $X(t)$ no $j$-ésimo "espaço de aproximação" $V_{j}$ e $\psi_{j, k}, k \in \mathbb{Z}$, no "espaço de detalhe" $V_{j-1} \ominus V_{j}$.

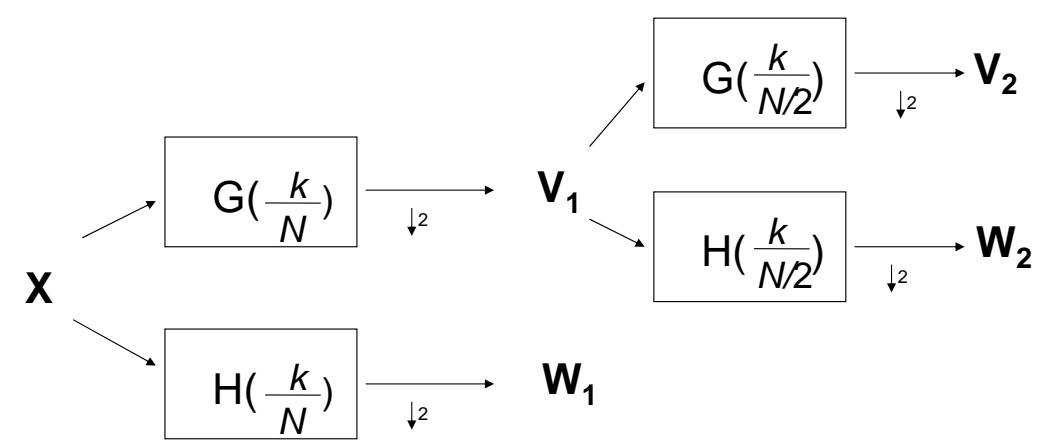

Figura 3.2: Diagrama de fluxo para a decomposição DWT [65].

\subsection{Transformada DWPT}

A transformada DWPT é uma generalização da transformada DWT e oferece uma análise mais flexível dos sinais. A unidade de análise da DWPT são formas de onda indexadas por três parâmetros naturalmente interpretados: posição, escala (como na DWT), e frequência. Para uma dada função wavelet ortogonal, um conjunto de bases pode ser gerado a partir da mesma. Cada uma destas bases oferece uma forma particular de codificar os sinais, preservando a energia global, e reconstruindo características exatas. As wavelet packets podem ser utilizadas para inúmeras expansões de um dado sinal, mas apenas uma destas expansões é selecionada como sendo a mais apropriada, segundo algum critério baseado, em geral, na entropia [56].

No procedimento de decomposição wavelet do algoritmo da pirâmide, o passo genérico divide o coeficiente de aproximação em duas partes. Após essa divisão, é obtido um vetor de coeficientes de aproximação e um vetor de coeficientes de detalhes, ambos em uma escala menos refinada. A informação perdida entre duas sucessivas aproximações é capturada nos coeficientes de detalhe. O passo seguinte consiste em dividir o novo vetor de coeficientes de aproximação; detalhes sucessivos nunca são analisados novamente. Na decomposição wavelet packet 
correspondente, cada vetor de coeficientes de detalhes também é decomposto em duas partes utilizando a mesma abordagem que na divisão do vetor de aproximações. Isso permite uma análise mais flexível: a árvore binária completa é produzida, conforme ilustrado na Figura 3.3, análoga à Figura 3.2 da análise wavelet correspondente.

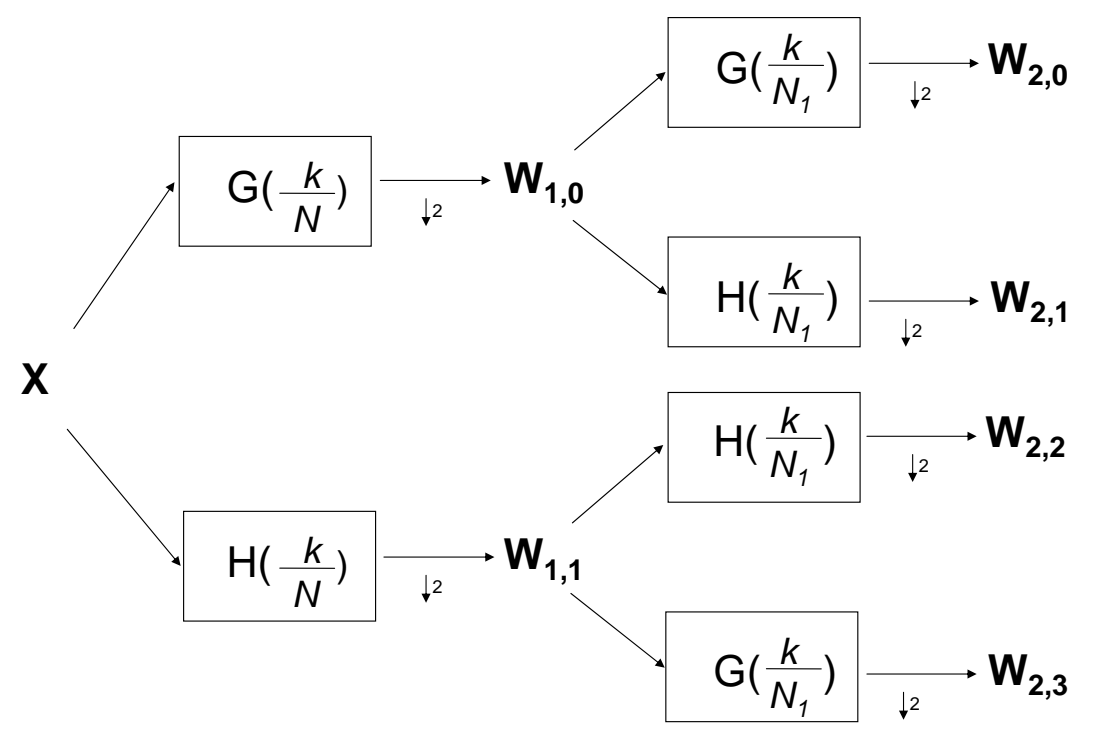

Figura 3.3: Diagrama de fluxo para a decomposição DWPT [65].

A Figura 3.4 ilustra os resultados da análise DWT (à esquerda) em comparação com os resultados da análise DWPT correspondente (à direita). O sinal analisado é um sinal oscilatório com modulação crescente sen $\left(250 \pi t^{2}\right)$, amostrado 512 vezes no intervalo $[0,1]$, com derivada da fase linear. Deve-se observar como, enquanto na análise DWT o comportamento da fase do sinal não é facilmente detectado, o correspondente gráfico da análise DWPT ilustra de forma bastante visual a fase linear crescente. Os gráficos foram obtidos através de exemplo de análise do Wavelet Toolbox ${ }^{\mathrm{TM}}$ do MATLAB ${ }^{\circledR}$.

O cálculo para a geração de wavelet packets é simples quando é utilizada uma wavelet ortogonal. Inicia-se com dois filtros de comprimento $2 N, h(n)$ e $g(n)$, correspondentes à wavelet.

Por indução, define-se a seguinte sequência de funções [66]:

$$
\left(W_{n}(x), n=0,1,2, \ldots\right)
$$

por

$$
\begin{aligned}
& W_{2 n}(x)=\sqrt{2} \sum_{k=0}^{2 N-1} h(k) W_{n}(2 x-k) \\
& W_{2 n+1}(x)=\sqrt{2} \sum_{k=0}^{2 N-1} g(k) W_{n}(2 x-k)
\end{aligned}
$$

onde $W_{0}(x)=\phi(x)$ é a função de escala e $W_{1}(x)=\psi(x)$ é a função wavelet. 

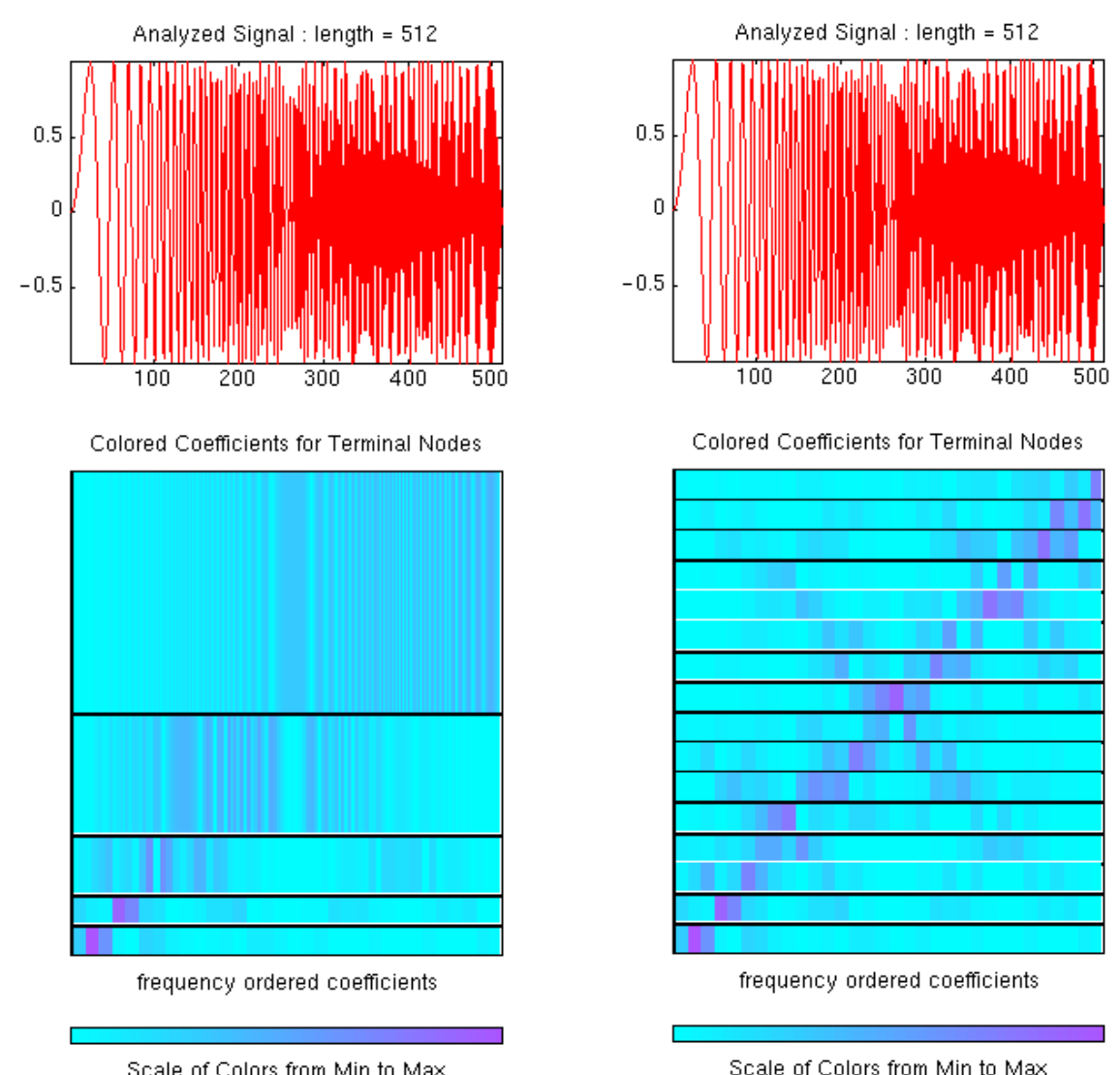

Figura 3.4: Comparação entre decomposições DWT (esq.) e DWPT (dir.) de um mesmo sinal [66].

Por exemplo, para a wavelet de Haar:

$$
\begin{aligned}
& N=1 \\
& h(0)=h(1)=1 / \sqrt{2} \\
& g(0)=-g(1)=1 / \sqrt{2}
\end{aligned}
$$

Desta forma, (3.17) podem ser reescritas como:

$$
\begin{aligned}
& W_{2 n}(x)=W_{n}(2 x)+W_{n}(2 x-1) \\
& W_{2 n+1}(x)=W_{n}(2 x)-W_{n}(2 x-1)
\end{aligned}
$$

$W_{0}=\phi(x)$ é a função de escala de Haar e $W_{1}=\psi(x)$ é a wavelet de Haar, ambas com suporte em $[0,1] . W_{2 n}$ pode ser obtido somando-se duas versões de $W_{n}$ reduzidas em escala por um fator de 2 com suportes distintos em $[0,1 / 2]$ e $[1 / 2,1]$, assim como $W_{2 n+1}$ pode ser obtido subtraindo-se as mesmas versões de $W_{n}$. A Figura 3.5 ilustra, para a wavelet de Haar, as funções $W$ para $n=0 \cdots 7$. A Figura 3.6 é análoga à Figura 3.5, mas para o caso de wavelet Daubechies 2 $(\mathrm{db} 2)$. 

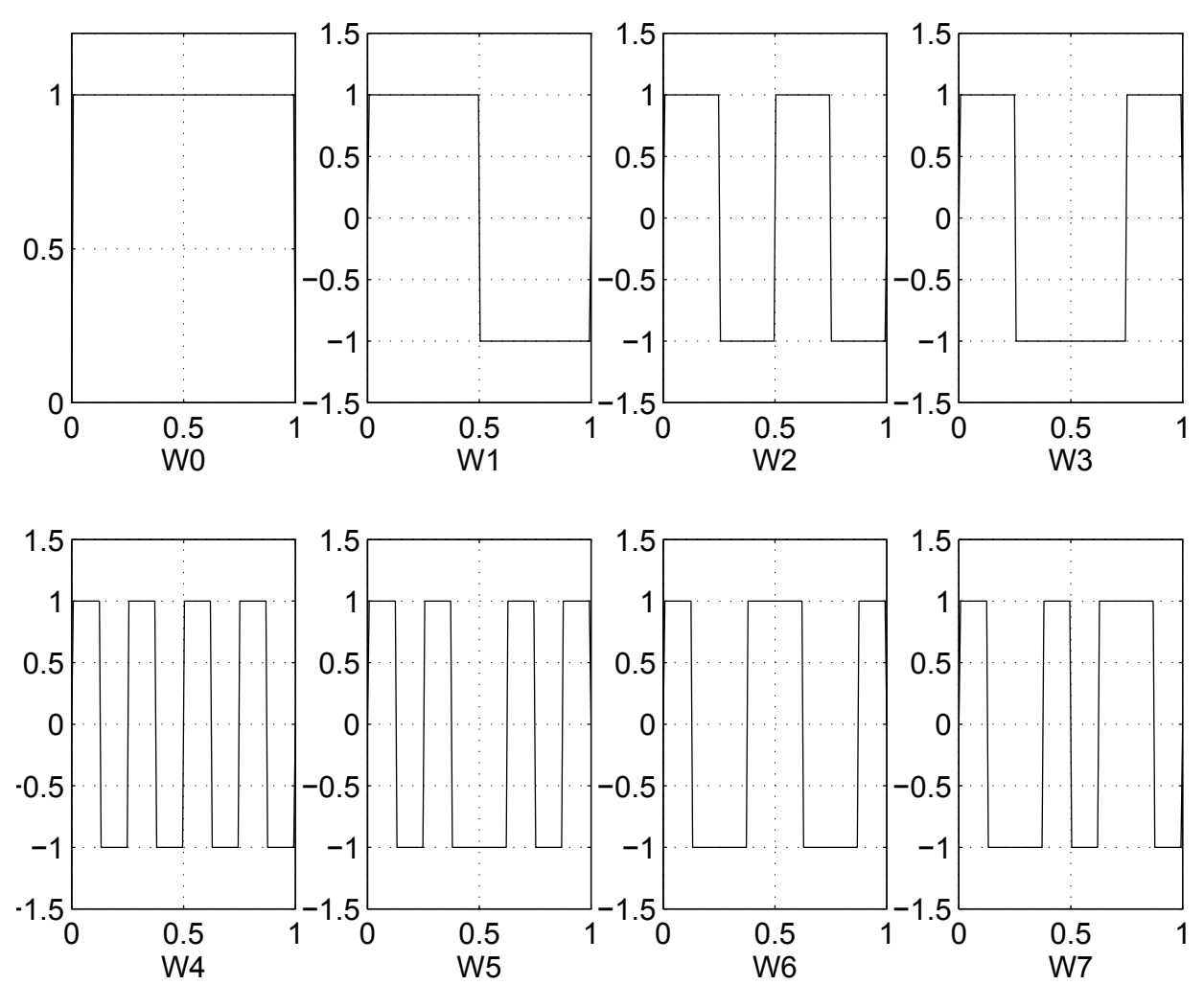

Figura 3.5: Wavelet packets de Haar [66].
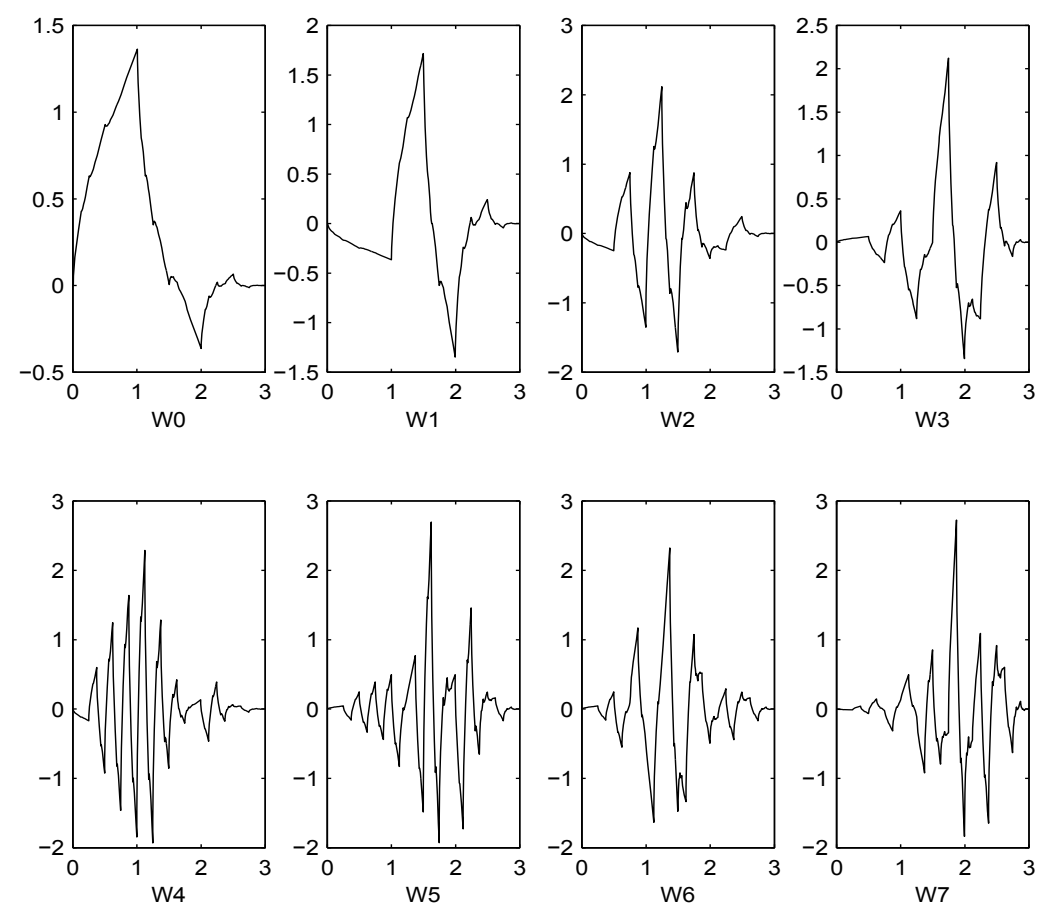

Figura 3.6: Wavelet packets db2 [66]. 


\subsubsection{Determinação da árvore DWPT}

Um sinal de comprimento $N=2^{L}$ pode ser expandido em $\alpha$ formas diferentes, onde $\alpha$ é o número de sub-árvores binárias da árvore binária completa de profundidade $L$. Como resultado, $\alpha \geq 2^{N / 2}$ [55, p.323]. A Figura 3.7 ilustra alguns exemplos de árvores que podem ser utilizadas para a decomposição DWPT de um determinado sinal (observe que a decomposição obtida através da árvore da Figura 3.7a corresponde à decomposição via DWT).

Como esse número de árvores possíveis pode ser muito grande, e uma vez que a enumeração explícita geralmente não é gerenciável, é interessante encontrar uma decomposição ótima em relação a um critério conveniente, calculável por um algoritmo eficiente.

Funções que observem uma propriedade do tipo aditiva são bastante apropriadas para uma busca eficiente de estruturas de árvores binárias. Critérios clássicos baseados em entropia atendem a essas condições e descrevem propriedades relacionadas à informação para uma representação fiel de um dado sinal. A entropia é um conceito comum em diversos campos, principalmente no de processamento de sinais. Abaixo serão listados quatro critérios diferentes de entropia [67]. Nas expressões que se seguem, $s$ é o sinal e $\left(s_{i}\right)$ são os coeficientes de $s$ em uma base ortonormal.

Conforme desenvolvimento em [66], a entropia $E$ deve ser uma função aditiva de custo tal que $E(0)=0$ e

$$
E(s)=\sum_{i} E\left(s_{i}\right)
$$

1. A entropia de Shannon não normalizada

$$
E_{1}\left(s_{i}\right)=-s_{i}^{2} \log s_{i}^{2}
$$

de forma que

$$
E_{1}(s)=-\sum_{i} s_{i}^{2} \log s_{i}^{2}
$$

com a convenção de que $0 \log 0=0$.

2. A concentração na norma $l^{p}$ com

$$
E_{2}\left(s_{i}\right)=\left|s_{i}\right|^{2}
$$




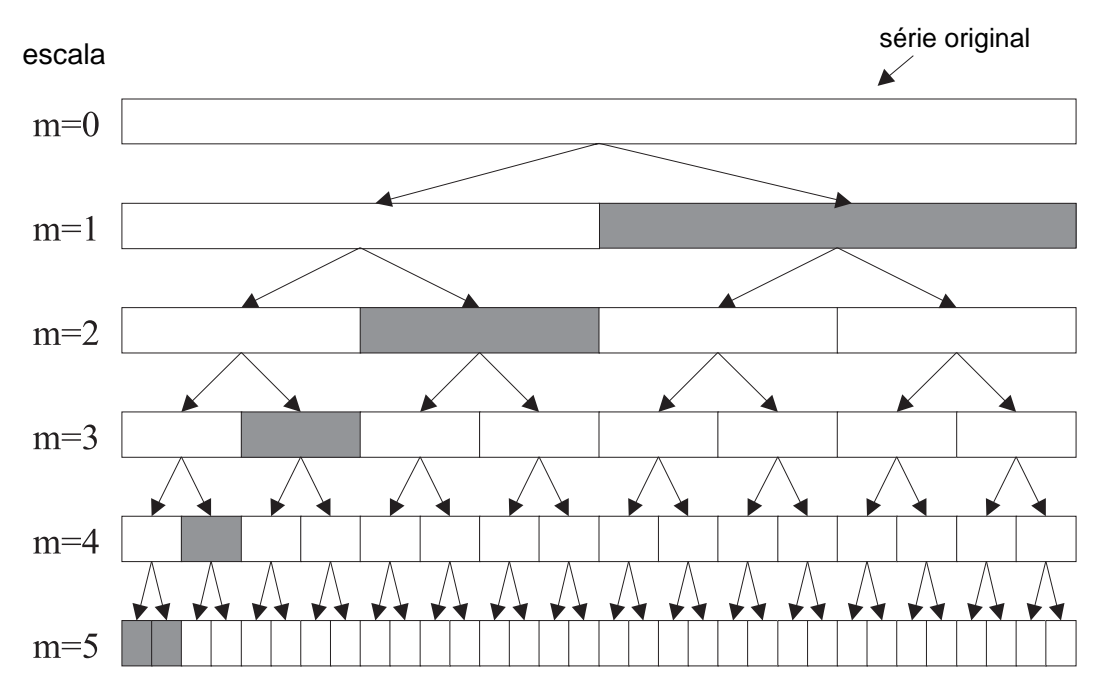

(a)

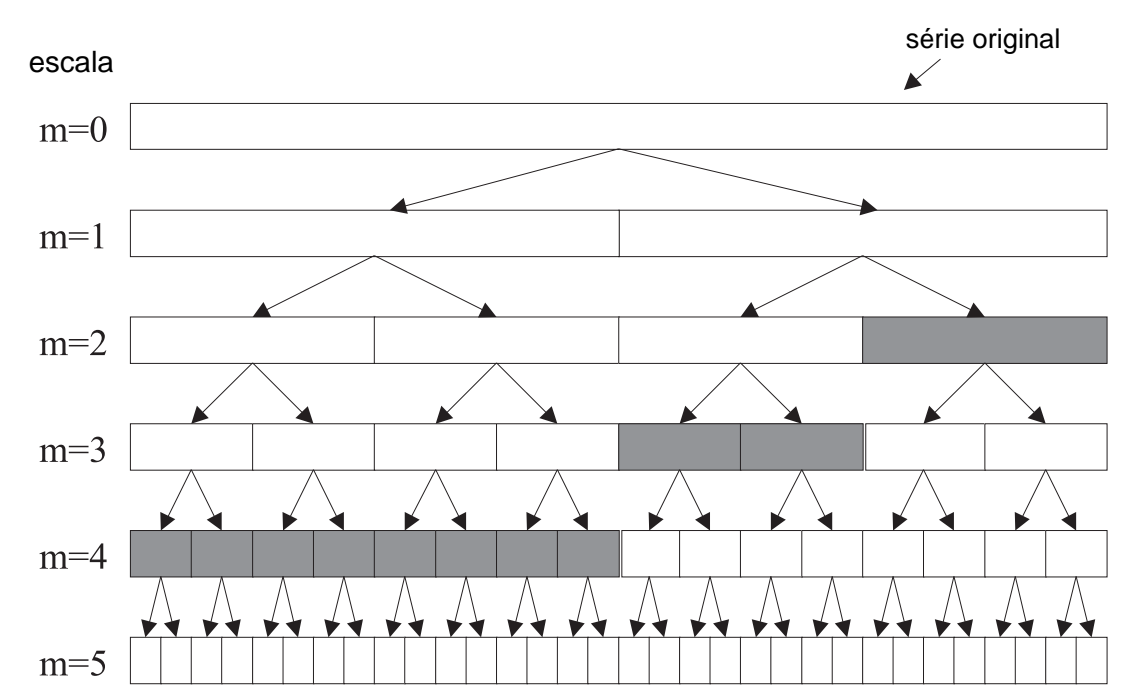

(b)

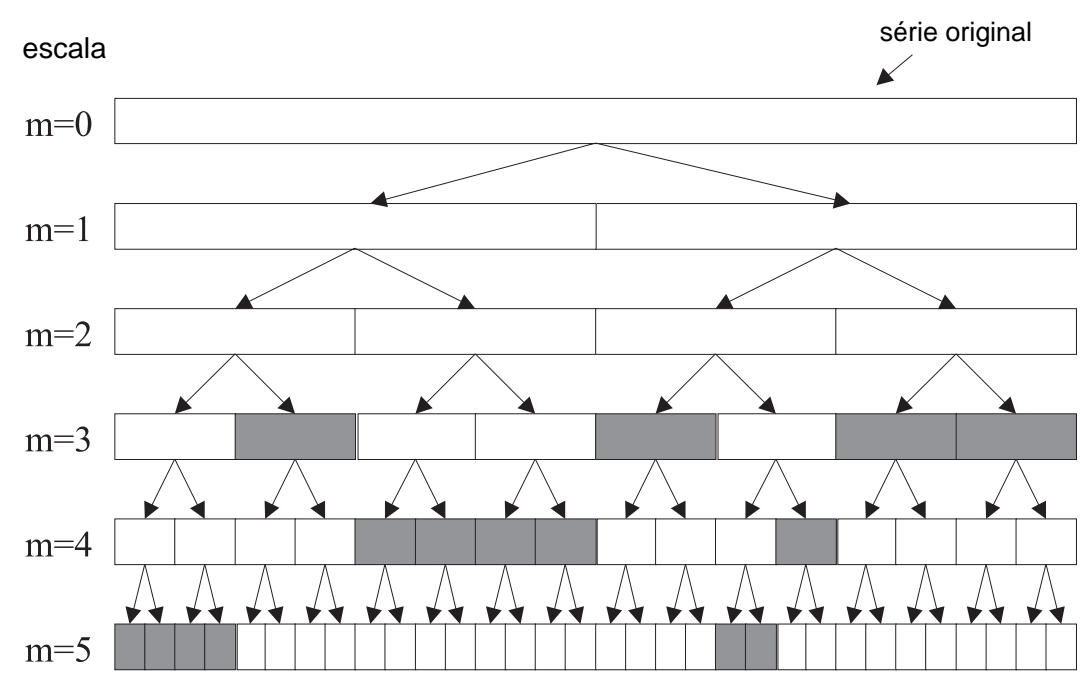

(c)

Figura 3.7: Exemplo de escolhas de árvores DWPT para análise de um sinal [56]. 
de forma que

$$
E_{2}(s)=\sum_{i}\left|s_{i}\right|^{p}=\|s\|_{p}^{p}
$$

3. O logaritmo da entropia da "energia"

$$
E_{3}\left(s_{i}\right)=\log s_{i}^{2}
$$

de forma que

$$
E_{3}(s)=\sum_{i} \log s_{i}^{2}
$$

com a convenção de que $\log 0=0$.

4. A entropia limiar

$$
E_{4}\left(s_{i}\right)=1 \text { se }\left|s_{i}\right|>\epsilon \text { e } 0 \text { caso contrário }
$$

de forma que $E_{4}(s)$ é o número de instantes no tempo em que o sinal é maior que o limiar pré-definido $\epsilon$.

\subsection{Coeficientes wavelet de processos auto-simi- lares e LRD}

O objetivo desta seção é descrever as propriedades estatísticas dos coeficientes wavelet de processos autossimilares e LRD, apresentando, em seguida, como a "invariância de escala" pode ser estudada no domínio wavelet. O desenvolvimento completo dos resultados abaixo pode ser encontrado em [10].

- Os coeficientes wavelet $d_{j, k}$ são os mesmos para $X(t)$ e $X(t)+P(t)$, onde $P$ é um polinômio de grau $N-1$, quando $\psi$ tem $N$ cumulantes nulos. Assim, a DWT é 'cega' a tendências polinomiais, uma vez que as mesmas são filtradas pela transformada.

- Suponha que o processo $\{X(t), t \in \mathbb{R}\}$ tenha incrementos estacionários. Então, para $j \in \mathbb{Z}$ fixo, $\left\{d_{j, k}, k \in \mathbb{Z}\right\}$ é uma sequência estacionária. Também pode ser demonstrado que se a wavelet $\psi$ tem $N$ cumulantes nulos, então $\left\{d_{X}(j, k), k \in \mathbb{Z}\right\}$ é estacionário se os incrementos de ordem $N$ de $X$ forem estacionários. De forma similar, $\left\{d_{X}(j, k), k \in \mathbb{Z}\right\}$ é estacionário se o processo $\{X(t), t \in \mathbb{R}\}$ for ele mesmo estacionário.

- Suponha que o processo $\{X(t), t \in \mathbb{R}\}$ seja autossimilar, com parâmetro de autossimilaridade $H$ (e, portanto, trata-se de um processo $H$-ss), de forma 
que $\forall c>0,\left\{c^{-H} X(c t), t \in \mathbb{R}\right\} \stackrel{d}{=}\{X(t), t \in \mathbb{R}\}$. Assim, para $j \in \mathbb{Z}$ fixo,

$$
d_{j, k} \stackrel{d}{=} 2^{j(H+1 / 2)} d_{0, k}
$$

como um processo em $k \in \mathbb{Z}$.

- Assumindo-se agora que $X(t)$ é um processo $H$-sssi, com $0<H<1$, média nula e variância infinita. Então $\mathbb{E} d_{j, k}=0$ e segue que:

$$
\mathbb{E} d_{j, k}^{2}=C 2^{j(2 H+1)}
$$

onde $C=\mathbb{E} d_{0,0}^{2}$. Tomando-se o logaritmo em ambos os lados de (3.29) resulta em uma função linear em $j$ cuja declividade é $2 H+1$. Esta observação é a base da estimação de $H$ pelo método de Abry-Veitch (conforme será detalhado na Seção 4.3)

Além da variância, a estrutura de covariância dos coeficientes wavelet de um processo $H$-sssi pode ser estudada. Para um dado $j$, os $\left\{d_{X}(j, k), k \in \mathbb{Z}\right\}$ são correlacionados, mas esta correlação tende rapidamente a 0 para um grande $\operatorname{lag}$ se $N$ for suficientemente grande:

$$
\mathbb{E} d_{j, k_{1}} d_{j, k_{2}} \leq C\left|k_{1}-k_{2}\right|^{2(H-N)},
$$

onde a constante $C$ depende de $j$. Em vista de (3.30), para evitar a dependência de longa duração para os coeficientes $d_{j, k}$, isto é, para garantir que $\sum_{k=0}^{\infty}\left|\mathbb{E} d_{j, k} d_{j, 0}\right|<\infty$ é preciso que seja escolhido $N>H+1 / 2$, ou seja, que $N=2$ pelo menos. A wavelet de Haar $(N=1)$ não permite descorrelação suficiente.

- Considere que $\{Y(t), t \in \mathbb{R}\}$ denote um processo estocástico estacionário de segunda ordem e que $r_{Y}$ e $g_{Y}$ sejam, respectivamente, a sua função de covariância e seu espectro. Tal processo é dito ser LRD se

$$
r_{Y}(\tau) \sim c_{r}|\tau|^{\gamma-1}, \tau \rightarrow+\infty, \gamma \in(0,1)
$$

ou se

$$
g_{Y}(v) \sim c_{g}|v|^{-\gamma}, v \rightarrow 0, \gamma \in(0,1)
$$

Adicionalmente, a variância dos coeficientes wavelet, para o caso LRD, reproduz o comportamento assintótico da lei de potência ${ }^{2}$ da definição de LRD:

$$
\mathbb{E} d_{j, k}^{2} \sim c_{g} 2^{j \gamma} \int_{\mathbb{R}}|v|^{-\gamma}|\tilde{\psi}(v)|^{2} d v, j \rightarrow+\infty .
$$

\footnotetext{
${ }^{2}$ Do inglês, "power-law".
} 
No caso LRD, esta relação (assintótica) é a base da estimação de $\gamma$ (ou $H$, se $\gamma=2 H-1$, com $1 / 2<H<1$ ). A covariância associada ao mesmo processo pode ser definida como

$$
\mathbb{E} d_{j, k} d_{j, k^{\prime}} \approx\left|k^{\prime}-k\right|^{\gamma-2 N-1},\left|k^{\prime}-k\right| \rightarrow+\infty
$$

que mostra que quanto maior $N$, menor a faixa de dependência.

Os resultados acima mostram que os coeficientes wavelet de processos autossimilares e LRD compartilham das mesmas propriedades fundamentais: i) estacionariedade para uma escala fixa, ii) ausência de dependência estatística de longa duração (cf. Equações (3.30) e (3.34)), e iii) reprodução no domínio wavelet das características que definem o fenômeno de invariância de escala (cf. Equações (3.29) e (3.33)). Deve ser enfatizado que estas propriedades resultam de uma correspondência profunda entre o fenômeno analisado - processos com invariância de escala - e a ferramenta de análise - análise wavelet ou de multirresolução. De forma mais precisa, estas propriedades baseiam-se no fato de que i) a wavelet mãe tem ao menos 1 cumulante nulo, e ii) as wavelets são construídas a partir da wavelet mãe usando-se o operador de dilação. Estas propriedadeschave dos coeficientes wavelet constituem a base para ferramentas de detecção, análise e estimação de processos com invariância de escala, conforme detalhado na Seção 4.3 . 


\section{Modelagem de tráfego fractal}

Este Capítulo apresenta os principais conceitos da modelagem de tráfego fractal. O Movimento Browniano e o Movimento Browniano fracionário são apresentados como motivação para o Ruído Gaussiano fracionário, principal modelo utilizado nas simulações deste trabalho. Métodos clássicos de estimação do parâmetro $H$, como o método de Whittle, são apresentados. Uma exposição do método de Abry-Veitch, baseado no espectro wavelet, conclui o Capítulo.

Ao leitor interessado em aprofundar-se nos conceitos da teoria de séries temporais, recomenda-se leitura das referências: [68], [69], [70], [71], [72], [73] e [74].

\subsection{Ruído Gaussiano fracionário}

Conforme desenvolvimento exposto em [3], o processo estacionário Ruído Gaussiano fracionário - ou Fractional Gaussian Noise (FGN) - $Y_{t}$, proposto por Mandelbrot e van Ness em 1968 [53], corresponde à primeira diferença (é o processo de incrementos) de um processo autossimilar $X_{t}$ denominado movimento Browniano fracionário de tempo discreto (discrete fractional Brownian motion - DFBM)[39],

$$
Y_{t}=X_{t}-B X_{t}=X_{t}-X_{t-1}=\nabla X_{t},
$$

em que $B$ denota o operador atraso unitário e $\nabla=(1-B)$ é o operador diferença. A DEP do DFBM é dada pela fórmula [49, pág. 280]

$$
P_{X}(f)=\sigma_{Y}^{2} C_{H} \sum_{j=-\infty}^{\infty} \frac{1}{|f+j|^{2 H+1}}
$$

em que $\sigma_{Y}^{2}$ é a potência do FGN, $C_{H}=\frac{\Gamma(2 H+1) \sin (\pi H)}{2 \pi^{2 H+1}}$ e $0<H<1$. De acordo com (4.2), a DEP do DFBM possui um pólo na origem, pois

$$
P_{X}(f) \propto|f|^{1-2 H}, \quad f \rightarrow 0
$$

O DFBM é um processo integrado de ordem 1 (portanto é não estacionário), 
porque a sua primeira diferença, o FGN, é estacionária. Como $Y_{t}=\nabla X_{t}$, o FGN e o DFBM estão relacionados pela função de transferência (na variável complexa z)

$$
H(z)=\frac{Y(z)}{X(z)}=1-z^{-1}
$$

A resposta em frequência é dada por

$$
H(f)=\left.H(z)\right|_{z=e^{j 2 \pi f}}=1-e^{-j 2 \pi f} .
$$

Como a relação entrada/saída em termos das DEPs é igual a [75, pág. 351]

$$
P_{Y}(f)=|H(f)|^{2} P_{X}(f),
$$

em que $|H(f)|^{2}$ é dado por,

$$
|H(f)|^{2}=G(f)=4 \sin ^{2}(\pi f),
$$

então,

$$
P_{Y}(f)=4 \sin ^{2}(\pi f) P_{X}(f)
$$

Assim, (4.2) e (4.8) mostram que a DEP do FGN é caracterizada por somente dois parâmetros: $\sigma_{Y}^{2}$ e $H$ (responsável pela forma do espectro). Além disso, é importante se ter em mente que o FGN é completamente especificado pela sua média e pela sua DEP, pois é Gaussiano.

Em [5], é mostrado que (4.8) pode ser reescrita na forma:

$$
P_{Y}(f)=A(f, H)\left[|2 \pi f|^{-2 H-1}+B(f, H)\right]
$$

em que $A(f, H)=2 \sin (\pi H) \Gamma(2 H+1)(1-\cos (2 \pi f))$ e $B(f, H)=\sum_{j=1}^{\infty}[(2 \pi j+$ $\left.2 \pi f)^{-2 H-1}+(2 \pi j-2 \pi f)^{-2 H-1}\right]$. Para pequenos valores de $f$ tem-se que $P_{Y}(f) \propto$ $|f|^{1-2 H}$.

Constata-se que o FGN apresenta sequência de autocovariância de acordo com (2.9), portanto trata-se de um processo exatamente autossimilar de segunda ordem quando $1 / 2<H<1$. Para $H=1 / 2$, o FGN reduz-se a um ruído branco Gaussiano. Quando $0<H<1 / 2$, o processo é SRD [3]. 


\subsection{Métodos clássicos de estimação do parâme- tro $H$}

\subsubsection{Estatística $\mathrm{R} / \mathrm{S}$}

Seja uma série temporal $x_{t}, t=1,2, \ldots, N$. Hurst [52] propôs o teste de memória longa conhecido como estatística Range Over Standard Deviation (R/S) ou rescaled adjusted range [73, 39], definido como (cf. [3]):

$$
Q_{N}=\frac{1}{\hat{s}_{N}}\left[\max _{1 \leq k \leq N} \sum_{j=1}^{k}\left(x_{j}-\bar{x}\right)-\min _{1 \leq k \leq N} \sum_{j=1}^{k}\left(x_{j}-\bar{x}\right)\right],
$$

onde $\hat{s}_{N}=\sqrt{\hat{C}_{0}}$.

Em seu estudo para a série dos níveis anuais mínimos do Rio Nilo, Hurst observou que o gráfico log-log de $R / S$ versus $N$ espalhava-se ao longo de uma reta com inclinação superior a $1 / 2$, ou seja, que $\log (R / S)$ versus $N$ apresentava um comportamento do tipo $C N^{H}$ (denominado efeito de Hurst), em que $C$ é uma constante e $1 / 2<H<1$ denota o parâmetro de Hurst. Tal descoberta contradizia o comportamento esperado para processos Markovianos, em que $R / S$ deve ter um comportamento assintótico do tipo $C N^{1 / 2}[39]$.

O comportamento assintótico do tipo $C N^{1 / 2}$ para o gráfico log-log de $R / S$ versus $N$ é justificado, de acordo com [73, pág. 262] e [39, pág. 82], pelo fato da estatística $N^{-1 / 2} Q_{N}$ convergir para uma variável aleatória bem definida (para $N \rightarrow \infty$ ) quando $\boldsymbol{x}_{t}$ é um processo Ruído Branco Gaussiano (RBG). Por outro lado, quando $\boldsymbol{x}_{t}$ é LRD, é a estatística $N^{-H} Q_{N}$ que converge para uma variável aleatória bem definida.

O comportamento assintótico acima observado é utilizado pelo método de estimação do parâmetro $H$ denominado análise R/S. O procedimento de estimação pode ser assim descrito $[73,39]$ :

1. primeiramente, calcula-se a estatística $R / S$ usando-se $N_{1}$ observações consecutivas da série, em que $N_{1}$ deve ser um número suficientemente grande;

2. em seguida incrementa-se o número de observações por um fator $f$; isto é, calcula-se $R / S$ sobre $N_{i}=f N_{i-1}$ amostras consecutivas para $i=2, \ldots, s$. Para se obter a estatística $R / S$ com $N_{i}$ observações consecutivas, pode-se dividir a série em $\left[N / N_{i}\right]$ blocos e obter-se $\left[N / N_{i}\right]$ valores, em que [.] denota a parte inteira de um número real; 
3. a regressão do plot log-log de todas as estatísticas $R / S$ versus $N_{i}, i=$ $1, \ldots, s$, produz uma estimativa do parâmetro $H$.

\subsubsection{Variance Plot}

O gráfico da variância (variance plot) é um método heurístico de estimação do parâmetro de Hurst. Beran demonstra [39] que a média amostral de uma série LRD decresce com o seu tamanho $N$ mais lentamente do que no caso tradicional (variáveis independentes ou não correlacionadas), conforme a equação

$$
\operatorname{Var}(\bar{x}) \approx c N^{2 H-2}
$$

em que $c>0$.

O procedimento de estimação pode ser assim descrito (cf. [3]):

1. Seja $k$ um número inteiro. Para diferentes $k$ pertencentes à faixa $2 \leq k \leq$ $N / 2$, e para um número $m_{k}$ suficiente de subséries de tamanho $k$, calcular as médias de $m_{k}$ amostras de tamanho $k, \bar{x}_{1}(k), \bar{x}_{2}(k), \ldots, \bar{x}_{m_{k}}(k)$ e a média global

$$
\bar{x}(k)=m_{k}^{-1} \sum_{j=1}^{m_{k}} \bar{x}_{j}(k) .
$$

2. Para cada $k$, calcular a variância da amostra de $m_{k}$ médias de amostras $\bar{x}_{j}(k), j=1,2, \ldots, m_{k}$ :

$$
s^{2}(k)=\frac{1}{m_{k}-1} \sum_{k=1}^{m_{k}}\left(\bar{x}_{j}(k)-\bar{x}(k)\right)^{2} .
$$

3. Representar num gráfico $\log s^{2}(k)$ versus $\log k$.

Para o caso de dependência de curta duração ou independência, espera-se que o coeficiente angular $2 H-2$ do plot seja igual a -1 .

\subsubsection{Método do Periodograma}

A DEP de um processo LRD pode ser aproximada pela expressão $C_{P}|f|^{1-2 H}$ quando $f \rightarrow 0$, conforme definição (2.1). Como a DEP pode ser estimada pelo periodograma, um log-log plot do periodograma versus frequência deve acompanhar uma reta com inclinação $1-2 H$ para frequências próximas de zero. Este procedimento de estimação é conhecido como método do periodograma. 
O estimador $\hat{P}_{x}(f)$ da DEP é obtido pelo método do periodograma [76], com janelamento de dados (data tapering, para redução de vazamento de potência) e suavização (smoothing, para redução da variabilidade de $\hat{P}_{x}(f)$ ). O periodograma é calculado via (cf. [3]):

$$
\hat{P}_{x}(f)=\frac{1}{N}|X(f)|^{2}
$$

sendo que tal definição não inclui o janelamento ou a suavização, para melhor compreensão da essência do estimador.

\subsubsection{Método de Whittle}

O estimador de Whittle também é baseado no periodograma e envolve a minimização da função [6]

$$
Q(\theta)=\int_{-0.5}^{0.5} \frac{\hat{P}_{x}(f)}{P_{x}(\theta, f)} d f
$$

em que $\hat{P}_{x}(f)$ denota o periodograma da série $x_{t}, P_{x}(\theta, f)$ é a DEP teórica do modelo $\operatorname{FARIMA}(p, d, q) \boldsymbol{x}_{t}$ na frequência $f$ e $\theta=[p, d, q]$ representa o vetor de parâmetros desconhecidos. Vide [77] e [78] para mais detalhes sobre a classe de modelos FARIMA, e [39] para maiores detalhes sobre método de estimação propriamente dito.

\subsection{Método wavelet de Abry-Veitch}

O chamado método de Abry-Veitch para estimação do parâmetro de auto-similaridade $H$ trata-se mais de um algoritmo de estimação do que um estimador como os apresentados na Seção 4.2. Isso porque, embora faça parte de tal método uma fórmula explícita (conforme estimadores já apresentados) para a estimação de $H$ (cf. Equação (2.10) do artigo [62]), é necessário que antes da aplicação do estimador seja realizada, por exemplo, uma fase de detecção das escalas em que está presente o comportamento invariante de escala.

Esta Seção apresentará os fundamentos para utilização do chamado 'espectro wavelet' para detecção do comportamento invariante de escala, bem como a fórmula do estimador baseado em tal espectro. A questão da determinação da faixa do espectro em que é verificado o comportamento invariante de escala é então apresentada para o caso específico de séries LRD. Por fim, é abordada a questão da fase de inicialização do método para o caso de estimação de séries discretas. 


\subsubsection{Diagramas em escala logarítmica}

De maneira geral, as variâncias dos processos wavelet $d_{j \text {, }}$ sobre todas as escalas $\left\{2^{j}\right\}$, quando tais processos são estacionários, são uma descrição de segundaordem do processo $X$, o que constitui uma forma de "espectro wavelet" [10]. Como visto na Seção 3.4, notavelmente na Equação (3.33), uma característica recorrente em modelos que apresentam invariância de escala é a progressão em lei de potência das variâncias em função da escala (dentro da faixa de invariância de escala). Isso motiva uma visão logarítmica deste espectro, onde uma linha reta sobre algum intervalo, caso presente, indicaria invariância de escala, com a declividade indicando o expoente de escala. Desta maneira, ao gráfico de

$$
s_{j} \equiv \log _{2} \mathbb{E} d_{j}^{2}
$$

em função de $j$ é dado o nome de diagrama em escala logarítmica exato - ou Logscale Diagram (LD) exato. Este 'espectro wavelet' é obtido calculando-se a integral (na prática, de forma numérica) que define as variâncias do processo em questão, e pode ser considerado como a 'assinatura' de tal processo.

A Figura 4.1a apresenta três exemplos de gráficos LD exatos para o modelo FGN com $H=0,8$, sendo que cada exemplo foi obtido com wavelets de Daubechies com cumulantes nulos com $N=1, N=3$ e $N=6$. Os espectros obtidos não são idênticos uma vez que cada um foi obtido com uma wavelet diferente (os espectros são específicos à família de funções utilizadas na análise). Entretanto, as curvas são muito similares na forma (sendo que, para o caso das wavelets de Daubechies, os espectros convergem ponto-a-ponto com o aumento de $N$ ), são muito semelhantes a linhas retas com exceção das pequenas escalas, e compartilham a mesma declividade assintótica $\gamma=2 H-1$ para $j$ grande, o que caracteriza a natureza LRD do FGN. Esta é uma importante característica do gráfico LD de processos invariantes de escala em geral: a escolha específica da wavelet de análise é irrelevante na faixa que contém invariância de escala.

Assim como o espectro de Fourier pode ser estimado a partir de dados, o LD exato também pode [10]. A Figura 4.1b apresenta novamente o LD exato para $N=3$ e mais 5 conjuntos de estimativas não viesadas de $y_{j}=\hat{s_{j}}$ sobrepostas no gráfico, obtidas de 5 realizações FGN com 10.000 amostras cada. Os intervalos de confiança Gaussianos com 95\% de nível de confiança, correspondentes à variabilidade de $y_{j}$, são exibidos como segmentos verticais centrados nos valores conhecidos de $s_{j}$. Estes LD exatos estimados, com intervalos de confiança, são chamados simplesmente de LD. Na prática, como o verdadeiro $\mathbb{E} y_{j}=s_{j}$ não é 
conhecido, os intervalos de confiança são considerados centrados nas estimativas $y_{j}$.

Pela natureza do operador de dilação que gera a base wavelet, o número $n_{j}$ de coeficientes de detalhe disponíveis para análise na oitava $j$ é diminuído pela metade a cada aumento de $j$ (desconsiderando-se o efeito de borda, que faz com que essa diminuição seja ainda maior). Assim, os intervalos de confiança aumentam monotonicamente com o aumento das oitavas $j$, conforme ilustrado na Figura 4.1b.

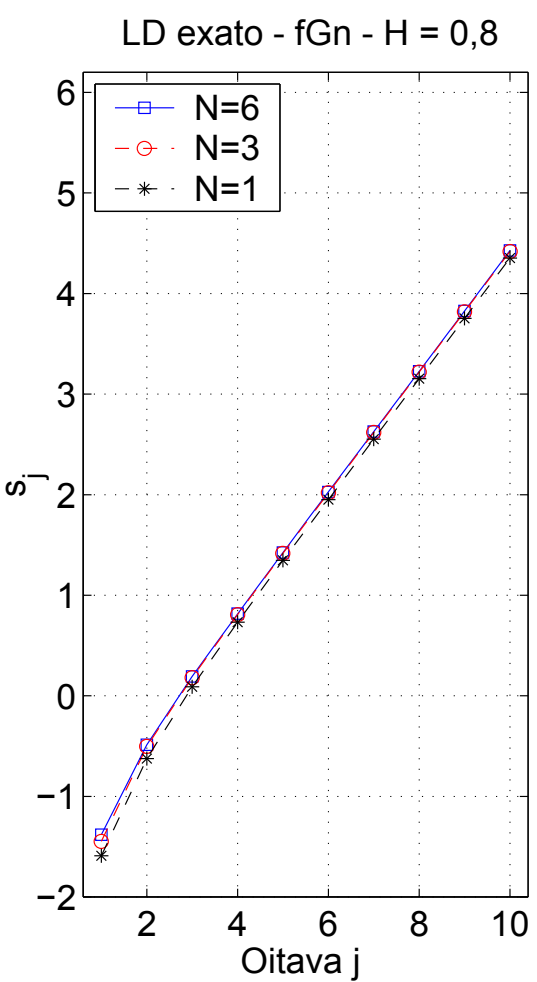

(a)

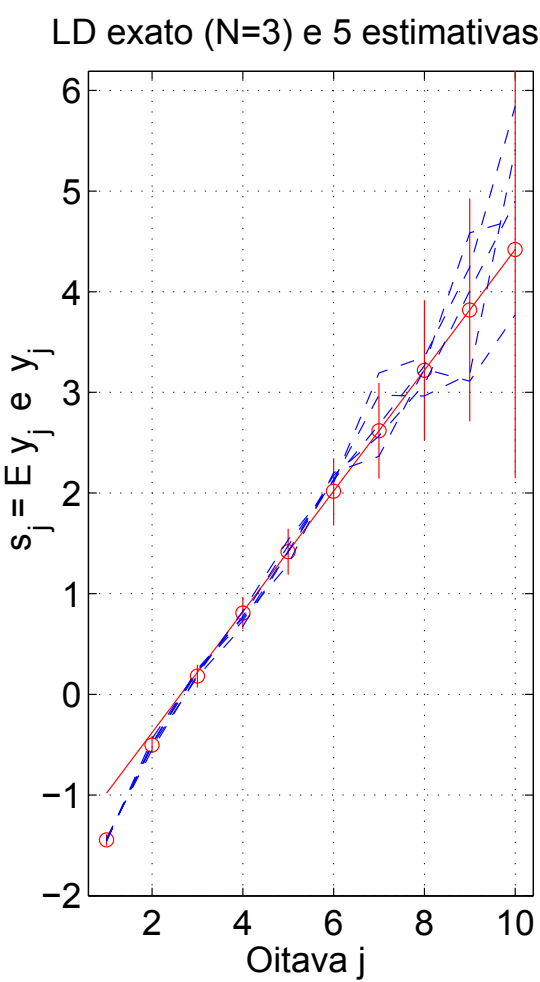

(b)

Figura 4.1: LD exato e estimativas do LD para um processo FGN com $H=0,8[10]$.

\subsubsection{Estimação com diagramas em escala logarítmica}

Considere o seguinte estimador não paramétrico, não viesado, da variância do processo de detalhes na escala $j$ (cf. [10]):

$$
\mu_{j}=\frac{1}{n_{j}} \sum_{k=1}^{n_{j}}\left|d_{j, k}\right|^{2} .
$$

O logaritmo desta variável seria um estimador de $s_{j}$, entretanto o mesmo seria viesado uma vez que a não linearidade do logaritmo significa que $\mathbb{E}[\log (\cdot)] \neq$ $\log (\mathbb{E}[\cdot])$. Para cancelar tal viés, é introduzida uma parcela de correção do viés 
$g(j)$, e define-se $y_{j}$ como

$$
y_{j}=\log _{2}\left(\mu_{j}\right)-g(j)
$$

Denota-se a variância de $y_{j}$ por $\sigma_{j}^{2}$, de onde os intervalos de confiança no LD podem ser calculados utilizando uma aproximação Gaussiana de $y_{j}$. Agora assuma que a faixa de invariância de escala $\left[j_{1}, j_{2}\right]$, onde está presente o comportamento invariante de escala e onde o LD exato é uma reta, tenha sido corretamente identificado. O índice $j_{1}$ é a chamada escala inferior de corte e o índice $j_{2}$ é a chamada escala superior de corte. A faixa $\left[j_{1}, j_{2}\right]$ poderia ser detectada na prática procurando-se por uma linha reta (consistente com os intervalos de confiança), no diagrama LD. Um estimador não viesado do expoente de invariância de escala com baixa variância pode ser obtido através de uma regressão linear ponderada sobre $y_{j}$ nesta faixa. Especificamente, definindo-se primeiramente as quantidades $S=\sum_{j=j_{1}}^{j_{2}} 1 / \sigma_{j}^{2}, S_{1}=\sum_{j=j_{1}}^{j_{2}} j / \sigma_{j}^{2}$ e $S_{2}=\sum_{j=j_{1}}^{j_{2}} j^{2} / \sigma_{j}^{2}$, o estimador $\hat{\gamma}$ de $\gamma$ é dado por

$$
\hat{\gamma}=\frac{\sum_{j=j_{1}}^{j_{2}} y_{j}\left(S_{j}-S_{1}\right) \sigma_{j}^{2}}{S S_{2}-S_{1}^{2}} \equiv \sum_{j=j_{1}}^{j_{2}} w_{j} y_{j},
$$

que é não viesado desde que o LD exato verdadeiramente alinhe-se sobre a faixa $\left[j_{1}, j_{2}\right]$.

O cálculo de $g(j)$ e de $\sigma_{j}^{2}$, em geral, não pode ser realizado de forma exata (cf. [10]). Entretanto, as fórmulas apresentadas a seguir, obtidas idealizando-se as fracas correlações dos coeficientes wavelet para a completa descorrelação, são aproximações excelentes no caso em que os coeficientes $d_{j, k}$ são Gaussianos:

$$
\begin{aligned}
g(j) & =\Gamma^{\prime}\left(n_{j} / 2\right) /\left(\Gamma\left(n_{j} / 2\right) \ln 2\right)-\log _{2}\left(n_{j} / 2\right) \sim \frac{-1}{n_{j} \ln 2}, \\
\sigma_{j}^{2} & =\zeta\left(2, n_{j} / 2\right) / \ln ^{2} 2 \sim \frac{2}{n_{j} \ln ^{2} 2}
\end{aligned}
$$

onde $\Gamma$ é a função gama, $\Gamma^{\prime}$ a sua derivada, e $\zeta(2, z)=\sum_{n=0}^{\infty} 1 /(z+n)^{2}$ uma função Zeta de Riemann generalizada. As fórmulas acima são uma boa aproximação mesmo para casos de dados não Gaussianos $[62,7]$.

A maior vantagem prática da metodologia acima descrita é que o mesmo procedimento é exatamente aplicável independentemente do tipo de invariância de escala em questão ( $H$-sssi, LRD, etc.). Até mesmo a escolha da wavelet é irrelevante, como já mencionado, desde que o número de cumulantes nulos da wavelet seja suficientemente alto para garantir a quase-descorrelação dos detalhes. Uma primeira questão, entretanto, na aplicação prática dos modelos invariantes de escala em dados é a detecção do fenômeno de invariância de escala, o que 
envolve a seleção da faixa $\left[j_{1}, j_{2}\right]$. Se para determinado conjunto de dados já foi decidido, por exemplo, que um modelo $H$-ss é aplicável, então certamente seria escolhido $j_{1}=1$ e $j_{2}$ como sendo o maior possível para o dado comprimento dos dados, e não há nada a ser decidido; mas, para outros tipos de comportamento invariante de escala, ambas as escalas inferior e superior de corte existem e devem ser identificadas. Esta questão será explorada no caso intermediário dos processos LRD, onde $j_{2}$ é o maior possível por definição, mas $j_{1}$ não está definido a priori e deve ser determinado de alguma forma durante a detecção/medição.

\subsubsection{Estimação de LRD}

Uma questão sutil, mas essencial, é o fato de que a seleção da escala de corte inferior $j_{1}$ para o caso LRD não é uma questão prática e de estimação, mas envolve questões mais profundas: a escala de corte não é definida nem mesmo para o diagrama LD exato (cf. [10]). Esta é uma consequência da definição assintótica da LRD, onde não se define uma frequência na qual o regime de invariância de escala inicia-se. Para resolver este problema, é preciso que seja adicionada uma nova definição. Não há uma escala de corte 'real' que esteja relacionada ao espectro $g_{X}(\nu)$ ou ao LD exato do processo somente. A escala de corte tem significado apenas com relação a algum outro critério, que o método de AbryVeitch determina que seja a qualidade de estimação. Especificamente, aplica-se a abordagem de estimação para cada $j_{1}$, e define-se um $j_{1}=j_{1}^{E Q M}$ 'verdadeiro' como aquele correspondente ao Erro Quadrático Médio (EQM) mínimo, onde o EQM é definido como

$$
\operatorname{EQM}(\hat{\gamma}) \equiv \mathbb{E}(\hat{\gamma}-\gamma)^{2}=(\mathbb{E} \hat{\gamma}-\gamma)^{2}+\operatorname{Var}(\hat{\gamma})
$$

De forma intuitiva está claro que, para $n$ fixo, conforme move-se para frequências menores com o aumento de $j_{1}$, o comportamento assintótico da linha reta é menos e menos 'poluído' por partidas em pequenas escalas, e portanto o viés de estimação decresce, ao custo de um aumento na variância devido ao efeito de perda de dados. Um critério EQM permite que um compromisso entre estes dois efeitos possa ser expresso economicamente, como ilustrado na Figura 4.2b para um modelo particular com forte SRD. Ao aumentar $n$, o balanço move-se rumo a valores maiores de $j_{1}$ na medida em que as variâncias dos $y_{j}$ decrescem com $n$, enquanto que o viés em cada $j$, que depende do LD exato somente, permanece constante. Assim $j_{1}^{E Q M}(n)$, longe de ser uma constante para um processo indicando onde a LRD 'realmente' inicia-se, é na verdade uma função não decrescente de $n$. 


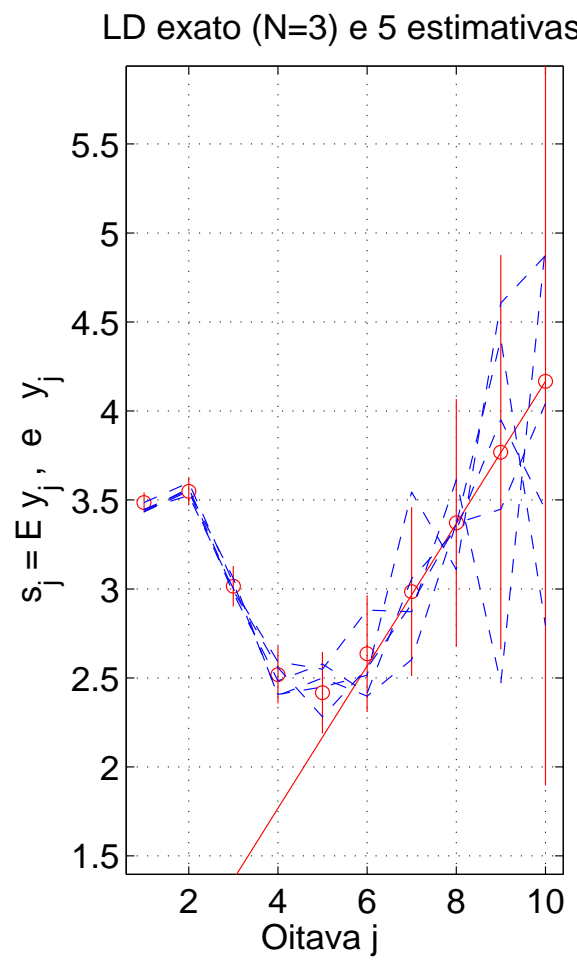

(a)

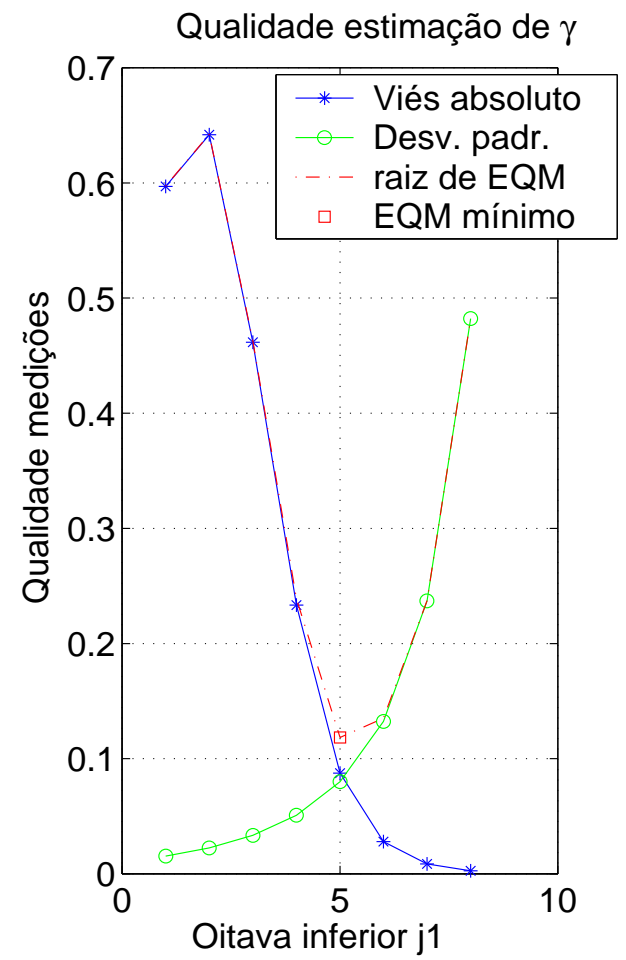

(b)

Figura 4.2: LD exato e $j_{1}^{E Q M}$ para um processo FARIMA com $H=0,7$ [10].

Poder-se-ia também descrever a escolha da escala de corte de outra maneira, como sendo o ponto de cruzamento entre a SRD e a LRD do processo. É interessante notar que mesmo para o processo LRD canônico, o FGN, a invariância de escala neste sentido não se estende a todas as escalas, e na verdade para $N=3$ e $n=10000$ a escala de corte definida pelo EQM é $j_{1}^{E Q M}=3$, o que é consistente com o desvio da inclinação assintótica em relação aos intervalos de confiança, conforme ilustrado na Figura 4.2b.

\subsubsection{Análise de dados discretos}

Uma questão que, geralmente, não é devidamente considerada é o fato de que a análise wavelet, incluindo a DWT, é definida para processos de tempo contínuo, e não para séries de tempo discreto como processos FARIMA e FGN discretos (cf. [10]). Isso é relevante uma vez que modelos de séries temporais em geral e muito dos dados reais enquadram-se nesta categoria. Conforme a Equação (3.14), para inicializar a sequência de aproximação inicial $a_{0, k}$ da DWT é necessário realizar o cálculo de uma sequência de integrais de tempo contínuo, um procedimento que não tem significado para dados discretos. Tipicamente, o que é feito quando deseja-se estudar o processo discreto $K(k)$ é atribuir $a_{0, k}=X(k)$, um procedimento que torna pouco claro o que está sendo de fato estudado, e introduz erros. 
O trabalho [79] mostrou como usar a DWT de uma forma bem definida para o estudo de propriedades de segunda ordem de processos discretos estacionários em sentido amplo, como os considerados nesta dissertação.

De forma simplificada, trata-se de realizar uma operação de convolução: $a_{0, k}^{\tilde{X}}=(X * I)(k)$, onde $I$ é um filtro discreto dado por

$$
I(m)=\int_{-\infty}^{+\infty} \sin \pi(t+m) \phi_{0}(t) / \pi\left(t_{m}\right) d t
$$

que depende unicamente da wavelet utilizada ( $I$ tem suporte infinito, mas na prática pode ser considerado como sendo relativamente pequeno). A pré-filtragem é fácil de ser realizada, e corrige erros que seriam, de outra forma, bastante significantes nas primeiras duas oitavas, mas desprezíveis em escalas menos refinadas. As séries do exemplo da Figura 4.1, que analisa FGN discreto, foram submetidas a essa filtragem, e portanto os valores $y_{j}$ são válidos para todas as oitavas. 


\section{Parte II}

Análise exploratória 


\section{Objetivos}

A Seção 1.1 apresentou a motivação para o estudo das características autossimilares e de dependência de longa duração do teletráfego de redes IP, bem como introduziu a importância de uma correta e precisa estimação do parâmetro de autossimilaridade.

Conforme também já exposto, o tráfego IP pode apresentar uma componente SRD além da usual componente LRD. Baseado em tal característica, Paxson [80] alertava para a necessidade de incorporação de componentes SRD em séries temporais de teletráfego sintetizadas, de forma que representem corretamente as estatísticas de séries de teletráfego reais.

Seguindo a linha de pesquisa sugerida por Paxson, o trabalho de Mello [81] inova ao sugerir a geração de séries de teletráfego com componentes LRD e SRD simultaneamente, sendo que a componente LRD é gerada através de um entre diversos métodos disponíveis (a ser determinado pelo usuário do gerador) e a componente SRD é incorporada através de uma filtragem Infinite Impulse Response (IIR).

Os artigos [82] e [31], dos quais o autor desta dissertação é um dos coautores, trazem um resumo do processo de tal geração, sendo que [31] introduz um importante resultado: a presença de componente SRD na série temporal pode causar um viés de estimação do parâmetro de autossimilaridade, viés este dependente do estimador utilizado. Adicionalmente, o texto [83], também de coautoria do autor desta dissertação, confirma o resultado anterior, ao evidenciar que, em alguns casos de presença simultânea de LRD e SRD, o método de estimação do parâmetro $H$ por Whittle pode mostrar-se inferior aos demais métodos de estimação usuais (tal comportamento é justificado no próprio artigo). É importante ressaltar que os trabalhos [31] e [83] utilizaram um número restrito de estimadores do parâmetro $H$, quais sejam: método de Whittle e método do periodograma (vide Seção $4.2)$.

Os resultados preliminares de tais trabalhos anteriores serão aqui estendidos 
e aprimorados com os seguintes objetivos:

1. verificar a possibilidade de melhoria na estimação do parâmetro $H$, na presença de SRD, através da aplicação do método de Abry-Veitch;

2. avaliar a qualidade da estimação do parâmetro $H$, na presença de SRD, variando-se os parâmetros ${ }^{1} N, j_{1}$ e $j_{2}$ do método de Abry-Veitch;

3. avaliar a qualidade da estimação do parâmetro $H$, na presença de não estacionariedades de $H$ e do nível de $\mathrm{SRD}$, variando-se os parâmetros $N, j_{1}$ e $j_{2}$ do método de Abry-Veitch;

4. verificar a possibilidade de melhoria na estimação do parâmetro $H$, na presença de SRD, através da estimação do espectro wavelet utilizando a DWPT.

O Capítulo 6 melhor elaborará os experimentos conduzidos para atingir os objetivos 1-3, referentes à exploração das características próprias do método de estimação de Abry-Veitch (conforme apresentado na Seção 4.3), enquanto o Capítulo 7 irá detalhar os experimentos realizados para atingir o objetivo 4, referente à exploração das características próprias da análise DWPT (conforme apresentado na Seção 3.3). Por fim, uma proposta de mecanismo de estimação de $H$ na presença de SRD é introduzida no Capítulo 8, com base nos resultados da análise exploratória dos Capítulos 6 e 7.

\footnotetext{
${ }^{1} \mathrm{O}$ número de amostras utilizadas para estimação através algoritmo de Abry-Veitch será referenciado no restante do texto como $N$ - o que não deve ser confundido com o número de cumulantes nulos, exposto ao longo da Fundamentação Teórica.
} 


\section{Método de Abry-Veitch}

\subsection{Análise preliminar}

A análise preliminar descrita a seguir tem como objetivo aprofundar os resultados observados no artigo [31] quanto à questão da estimação do parâmetro $H$ na presença de SRD.

\subsubsection{Condições experimentais}

A introdução de SRD no artigo [31] foi realizada através de um único filtro, aplicado em cada uma das 27 realizações obtidas (3 realizações para cada um dos 9 valores do parâmetro $H)$.

Para melhor generalização dos resultados a serem obtidos, foi expandido tanto o número de gerações das séries bem como a gama de filtros para introdução de SRD. Para tanto, foi utilizado o gerador de tráfego de Mello [81] conforme parâmetros arbitrados e abaixo descritos, totalizando 72 realizações sem adição de SRD:

- $H_{\text {gen }}$ : de 0,55 a 0,95 , com incrementos de 0,05

- número de realizações para cada valor de $H_{g e n}: 8$

- número de iterações (reconstrução com o algoritmo da Pirâmide): 12

- ordem do filtro de Daubechies: 1 (Haar)

- modelo de geração: FGN

- amostras por realização: 2048

A introdução de SRD, também conforme a metodologia definida em [81], foi feita tomando-se por base o mesmo filtro utilizado no artigo [31], definido como:

$$
S_{\text {orig }}(f)=\frac{1}{1-0,3 e^{-j 2 \pi f}+0,4 e^{-j 4 \pi f}}
$$


O filtro descrito pela função de transferência (6.1), com frequência central $f_{0} \approx 0,4 \pi \mathrm{rad} /$ amostra, foi transladado em frequência para frequências centrais de aproximadamente $0,1 \pi \mathrm{rad} /$ amostra a $0,9 \pi \mathrm{rad} /$ amostra, com incrementos de $0,1 \pi \mathrm{rad} /$ amostra. Adicionalmente, para cada uma das frequências centrais citadas, foi feita também a filtragem com filtros com ganho de $-3 \mathrm{~dB}$ e $+3 \mathrm{~dB}$ em relação ao filtro original, totalizando 1728 realizações com componentes SRD.

A totalidade das 2016 realizações acima descritas foi submetida ao algoritmo de estimação de Abry-Veitch. Após tal estimação ter sido realizada, o conjunto de variáveis abaixo descritas foi armazenado para análise ${ }^{1}$ :

- escala mínima do espectro wavelet utilizada para estimação $\left(j_{\min }\right)$;

- escala máxima do espectro wavelet utilizada para estimação $\left(j_{\max }\right)$;

- estimativa do valor de $H$;

- intervalo de confiança da estimativa do valor de $H$;

- escala mínima ótima para estimação de $\mathrm{H}\left(j_{\min }^{E Q M}\right)$, conforme definido em 4.3.

Considerando-se que, para as séries analisadas, o valor de $j_{\min }$ e $j_{\max }$ poderia variar entre 1 e 8, com diferença mínima de 2 escalas, conclui-se que para cada realização foram realizadas 36 estimativas para cada uma destas combinações de $j$, totalizando 72576 estimativas por tal método.

A estimação acima descrita foi realizada através do software MATLAB ${ }^{\circledR}$. Tal procedimento foi complementado através do software S-PLUS ${ }^{\circledR}$, que possibilitou obter as seguintes estatísticas:

- função de autocorrelação $\rho_{X}(m)$ (ACF - Autocorrelation Function);

- DEP $P_{X}(f)$;

- assimetria $A\left(X_{t}\right)$, que representa a concentração dos valores em um dos extremos da distribuição;

- curtose $K\left(X_{t}\right)$, que indica o grau de achatamento de uma densidade de probabilidade com relação à normal;

\footnotetext{
${ }^{1}$ Para um melhor entendimento da relação dos parâmetros $j_{1}$ e $j_{2}$ do algoritmo de AbryVeitch como sendo delimitadores do espectro wavelet, os mesmos serão referenciados no restante do texto, respectivamente, como $j_{\min }$ e $j_{\max }$.
} 
- $H$, pelos métodos de Whittle, do periodograma e do periodograma suavizado;

- $J B$, de Jarque e Bera, para teste de normalidade.

A Seção 6.1.2 detalha a análise realizada sobre os resultados obtidos conforme acima descrito ${ }^{2}$.

\subsubsection{Resultados obtidos}

Os resultados apresentados a seguir são uma breve compilação dos resultados mais relevantes que foram obtidos. Apenas os gráficos mais representativos são apresentados, sem prejuízo de generalização dos resultados.

A Figura 6.1 apresenta os gráficos da análise espectral para uma das realizações originais (sem SRD) para três valores distintos de $H$. Enquanto no periodograma suavizado é possível observar o comportamento aproximadamente $1 / f$ próximo à origem, no espectro wavelet é possível observar o ajuste da reta cuja inclinação corresponde à estimativa do parâmetro $H$.

Cada uma das realizações puramente LRD obtidas foram submetidas à filtragem descrita na Seção 6.1.1. Para uma melhor visualização dos resultados, a Figura 6.2 apresenta os gráficos da resposta em frequência de três dos filtros utilizados para adição de SRD às séries originais.

O filtros representados na Figura 6.2 foram utilizados para adição de SRD nas séries originais analisadas na Figura 6.1. A correspondente análise (periodograma suavizado e espectro wavelet) é apresentada, para cada filtragem, nas Figuras 6.3, 6.4 e 6.5. Os efeitos da SRD (evidenciados pelas setas vermelhas nos gráficos das Figuras 6.3-6.5) são notoriamente visíveis: no periodograma suavizado, corresponde à aparente 'corcova' em torno da respectiva frequência central do filtro; no espectro wavelet, observa-se uma sensível alteração do espectro correspondente às escalas mais rápidas $(j \leq 4)$.

A Figura 6.6 ilustra diversos casos de estimação de $H$ de forma comparativa entre os estimadores de Whittle e de Abry-Veitch. A Figura 6.6a, em particular, ilustra as estimativas obtidas para as séries originais sem adição de SRD: enquanto as estimativas por Whittle visualmente se mantêm alinhadas com a linha tracejada, que representa o ponto ótimo de $H_{\text {est }}$ (valor estimado do parâmetro

\footnotetext{
${ }^{2}$ Ressalta-se que, embora todos os parâmetros/estimativas gerados tenham sido armazenados em uma matriz única para posterior análise complementar, nem todos foram considerados na presente análise exploratória (os demais serão analisados em profundidade oportunamente).
} 


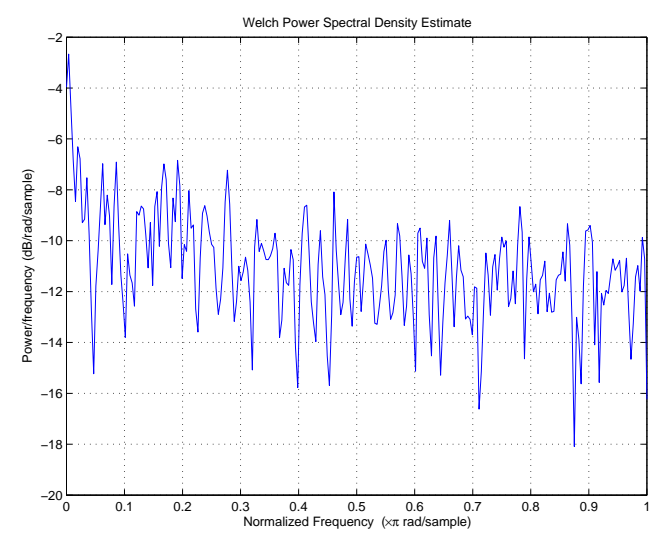

(a) Periodograma suavizado $(H=0,6)$

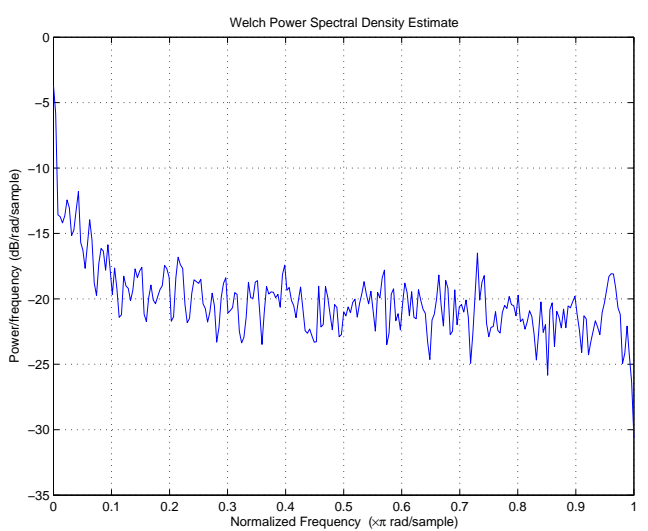

(c) Periodograma suavizado $(H=0,75)$

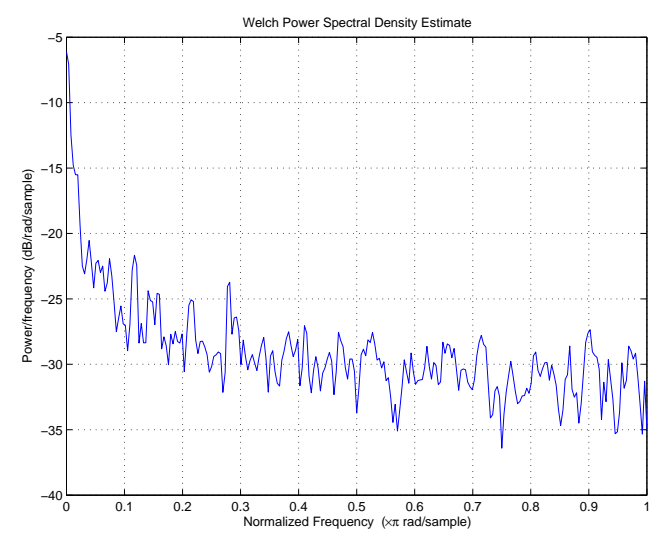

(e) Periodograma suavizado $(H=0,9)$

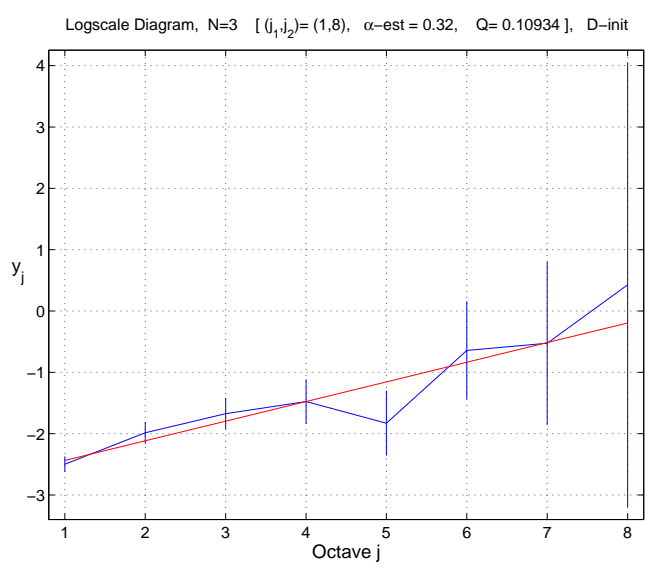

(b) Espectro wavelet $(H=0,6)$

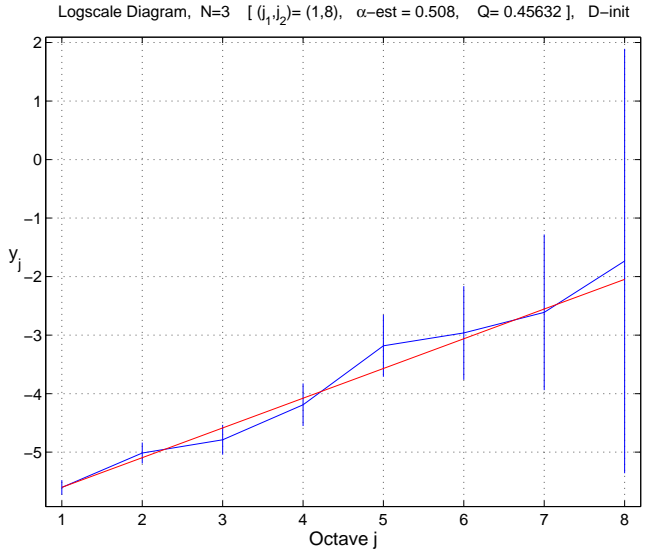

(d) Espectro wavelet $(H=0,75)$

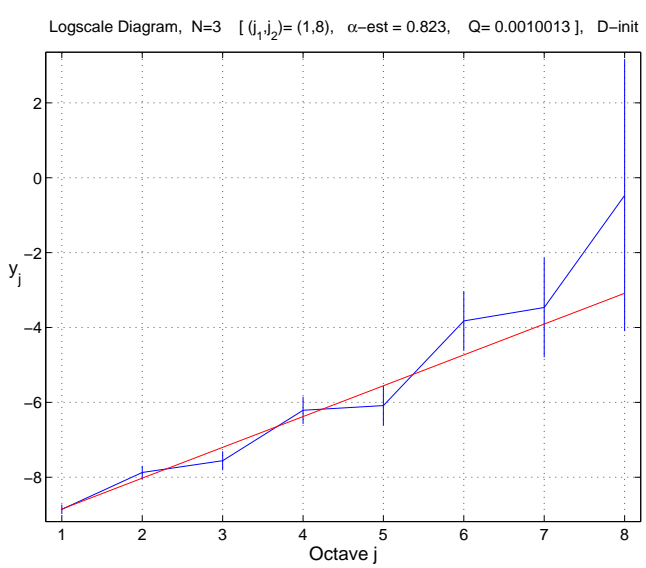

(f) Espectro wavelet $(H=0,9)$

Figura 6.1: Análise espectral das séries originais sem SRD. 

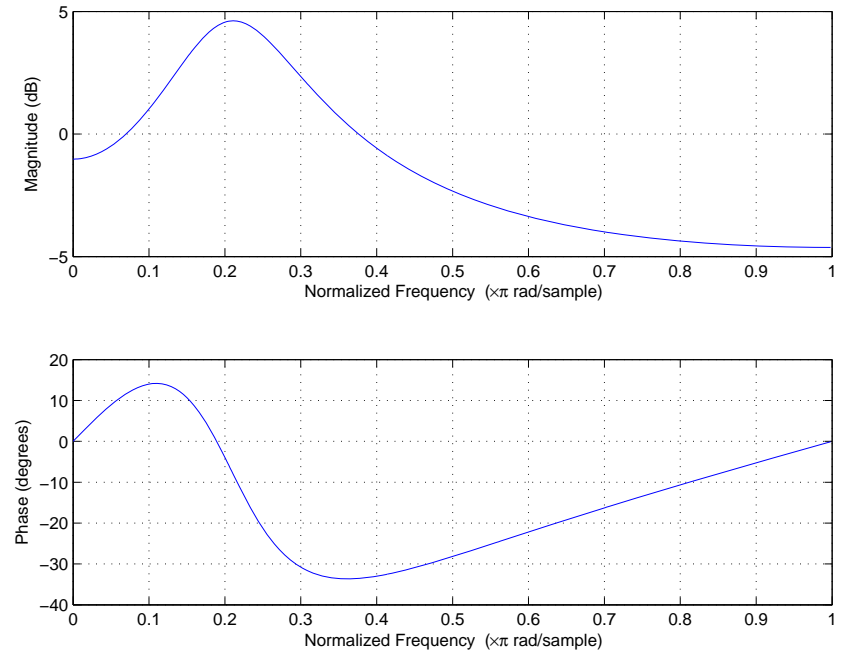

(a) $f_{0} \approx 0,2 \pi \mathrm{rad} /$ amostra
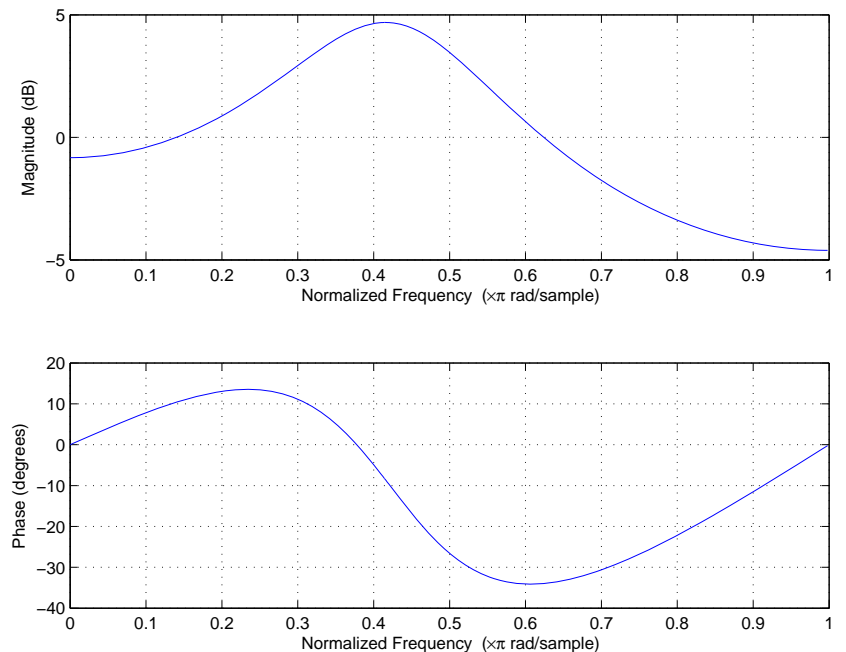

(b) $f_{0} \approx 0,4 \pi \mathrm{rad} /$ amostra
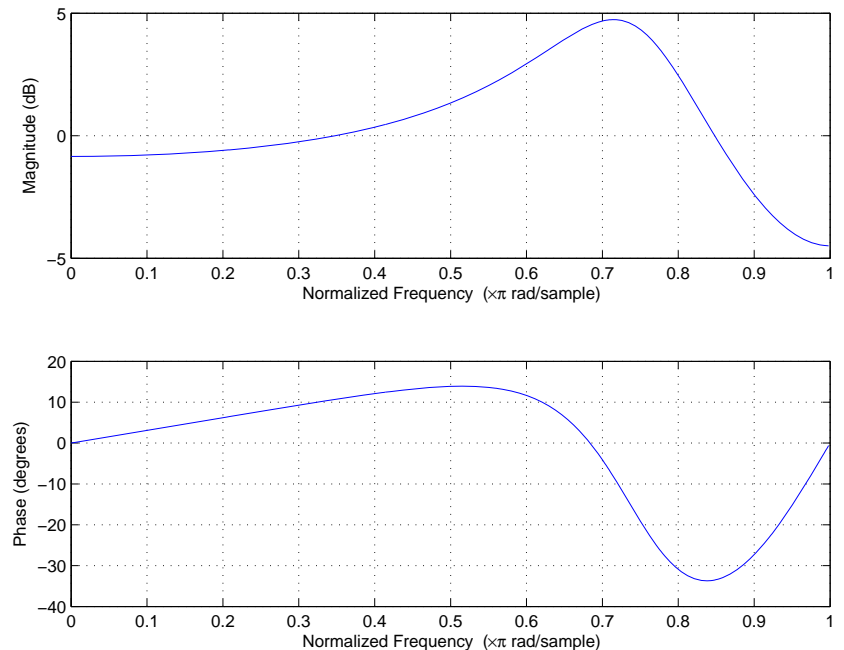

(c) $f_{0} \approx 0,7 \pi \mathrm{rad} /$ amostra

Figura 6.2: Resposta em frequência dos filtros IIR utilizados para adição de SRD. 


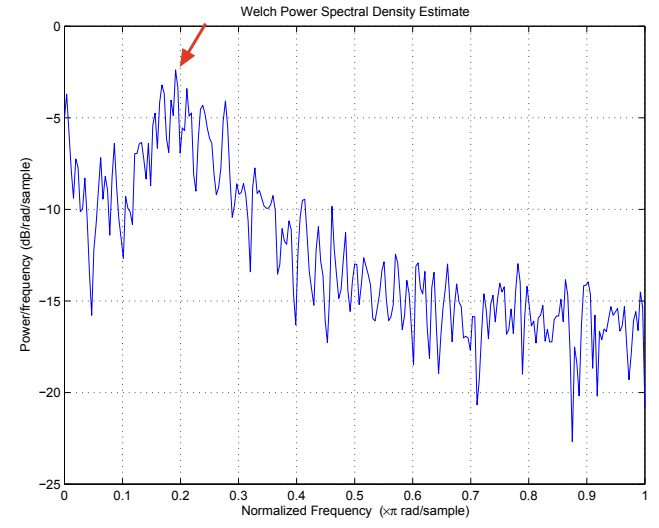

(a) Periodograma suavizado $(H=0,6)$

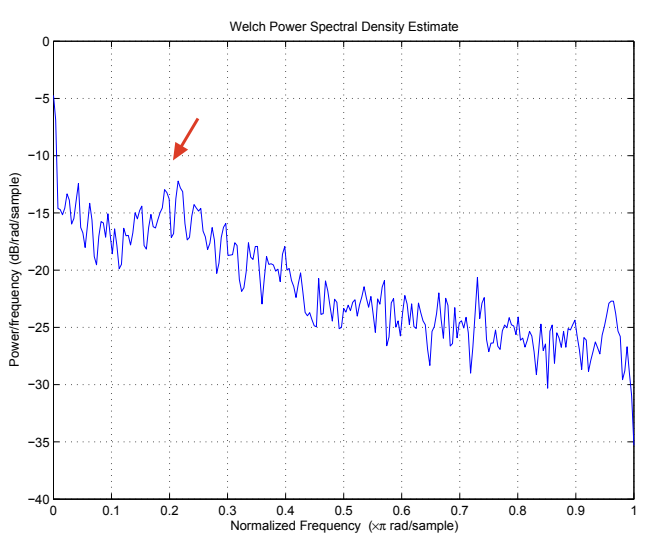

(c) Periodograma suavizado $(H=0,75)$

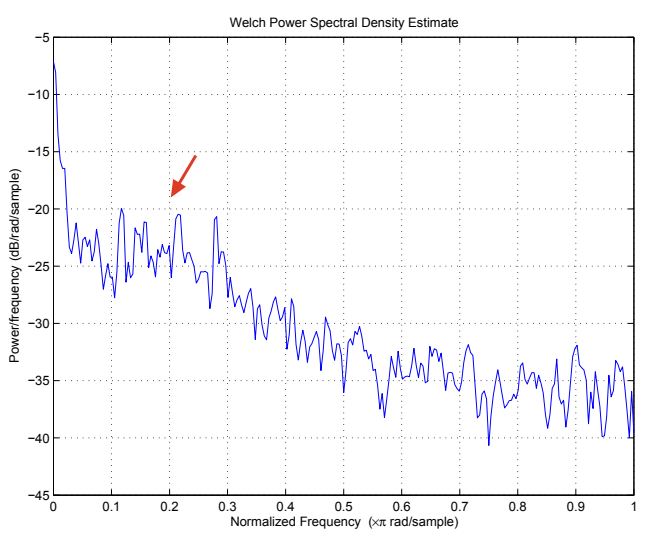

(e) Periodograma suavizado $(H=0,9)$

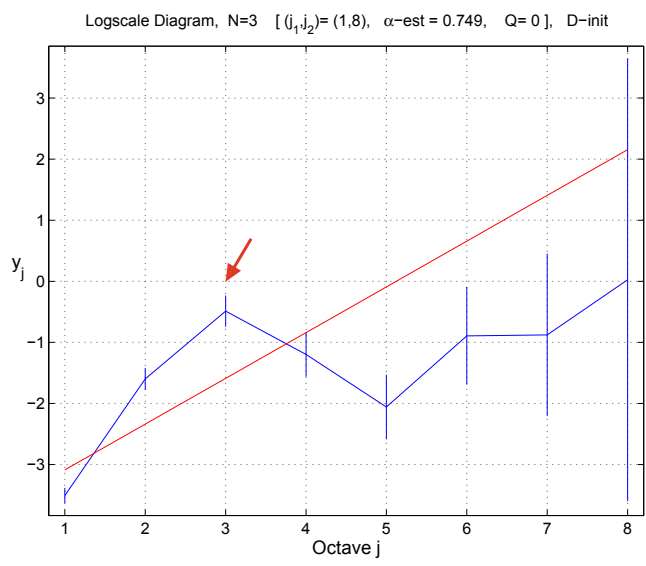

(b) Espectro wavelet $(H=0,6)$

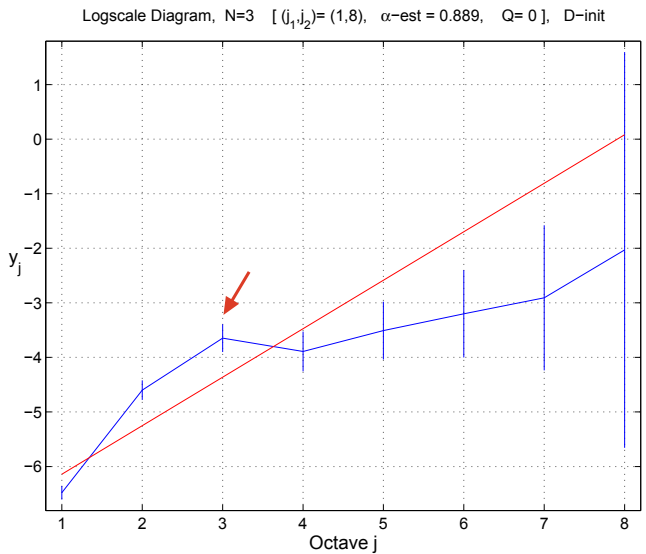

(d) Espectro wavelet $(H=0,75)$

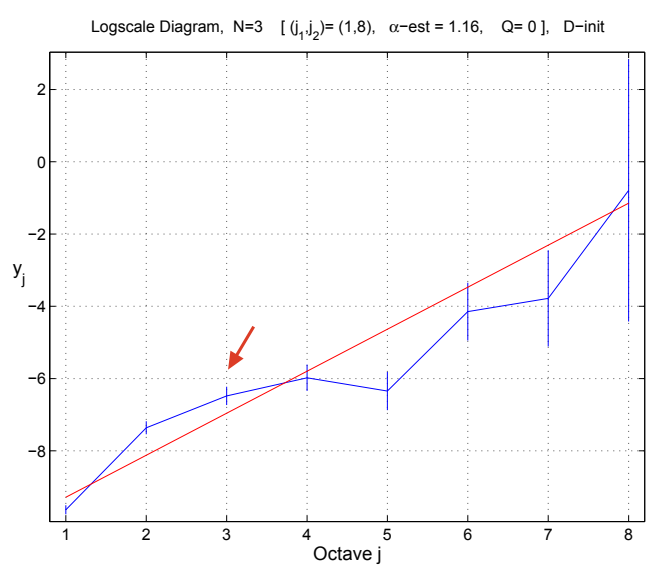

(f) Espectro wavelet $(H=0,9)$

Figura 6.3: Análise espectral das séries com $\operatorname{SRD}$ (filtragem com $f_{0} \approx 0,2$ ). 


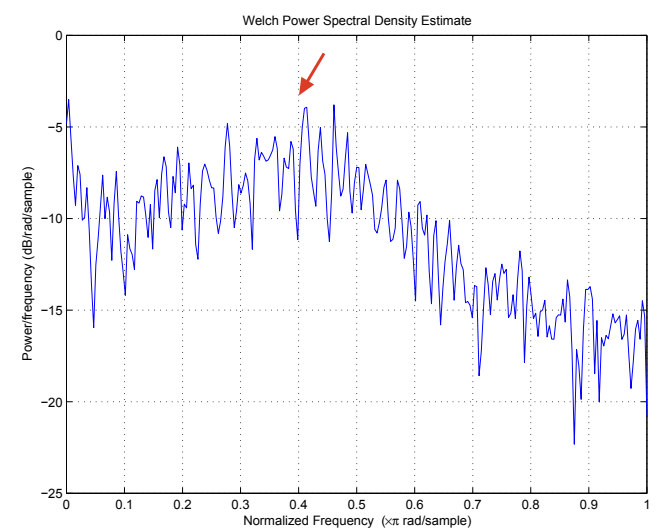

(a) Periodograma suavizado $(H=0,6)$

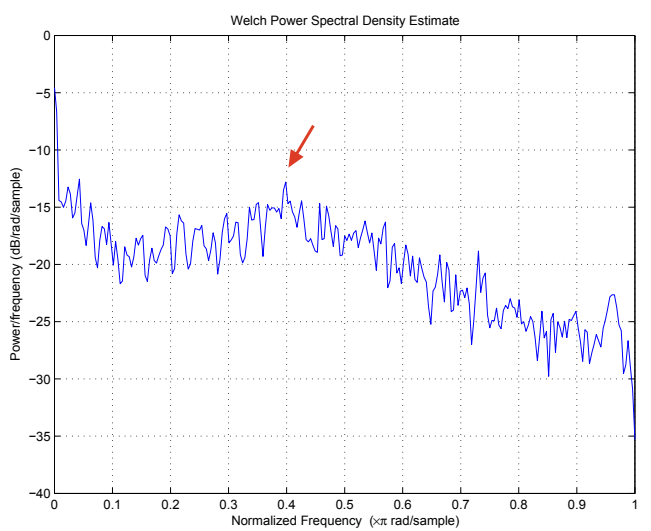

(c) Periodograma suavizado $(H=0,75)$

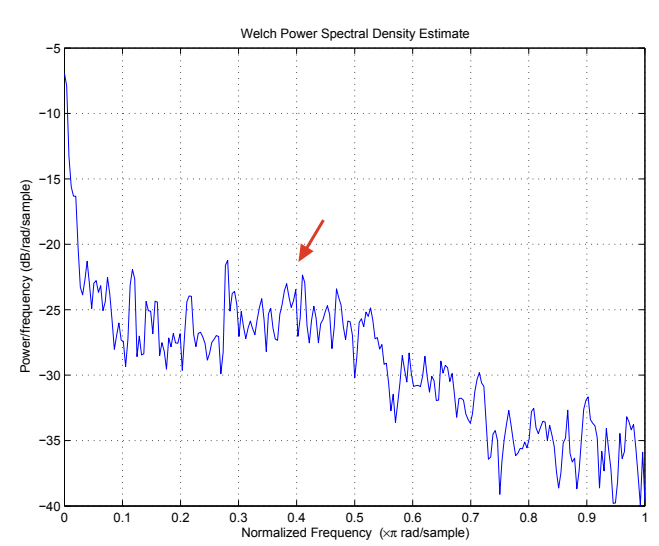

(e) Periodograma suavizado $(H=0,9)$

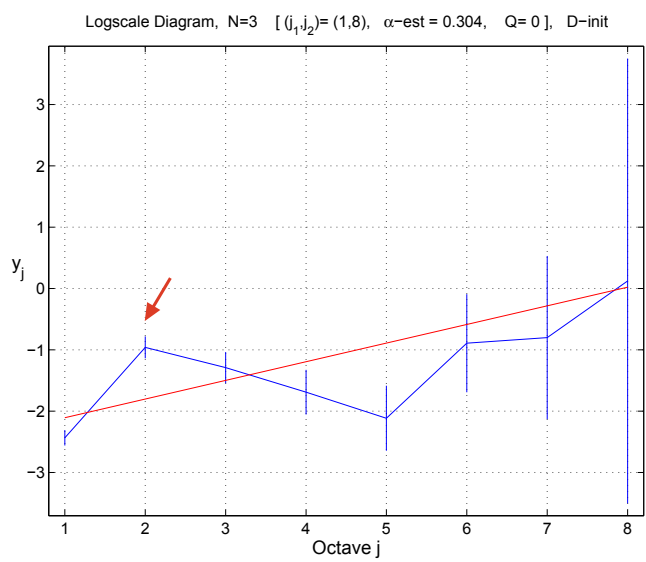

(b) Espectro wavelet $(H=0,6)$

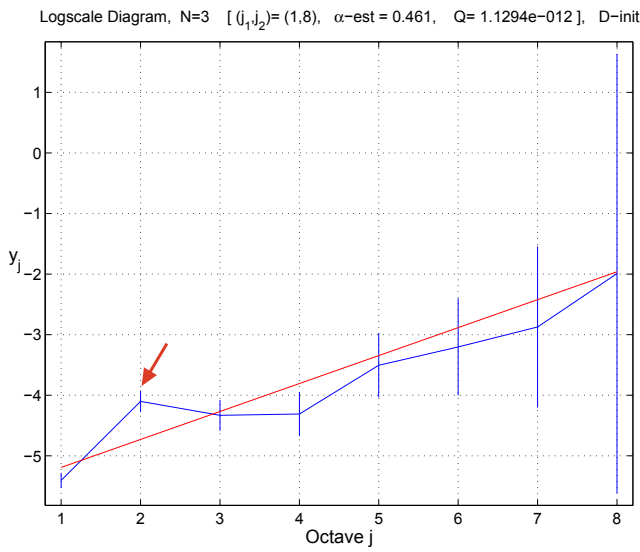

(d) Espectro wavelet $(H=0,75)$

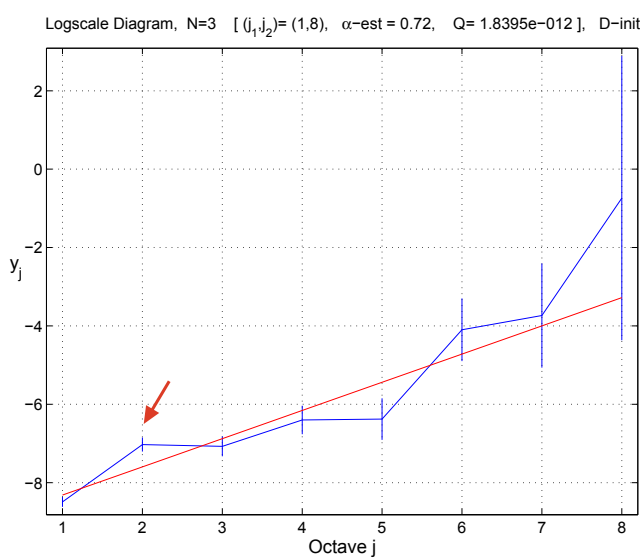

(f) Espectro wavelet $(H=0,9)$

Figura 6.4: Análise espectral das séries com SRD (filtragem com $f_{0} \approx 0,4$ ). 


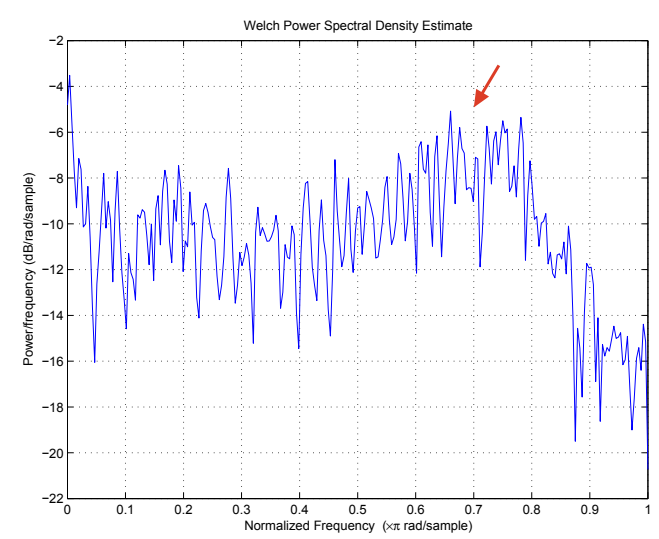

(a) Periodograma suavizado $(H=0,6)$

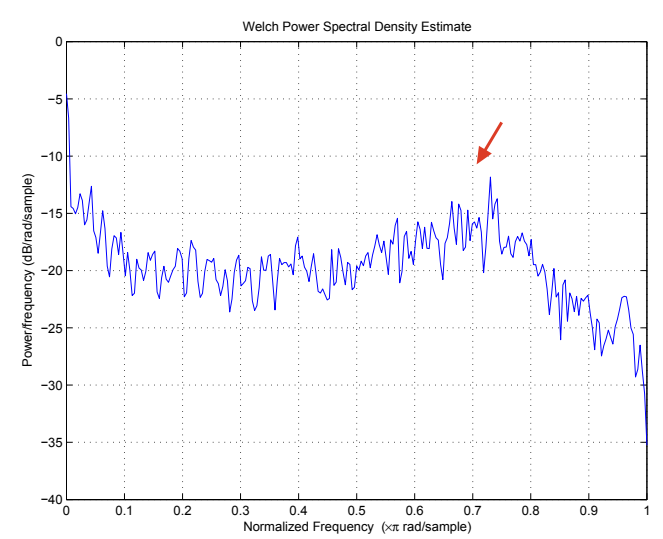

(c) Periodograma suavizado $(H=0,75)$

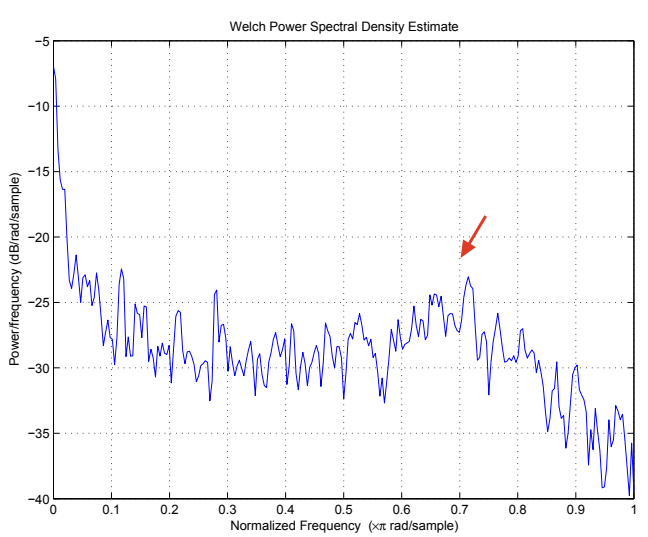

(e) Periodograma suavizado $(H=0,9)$

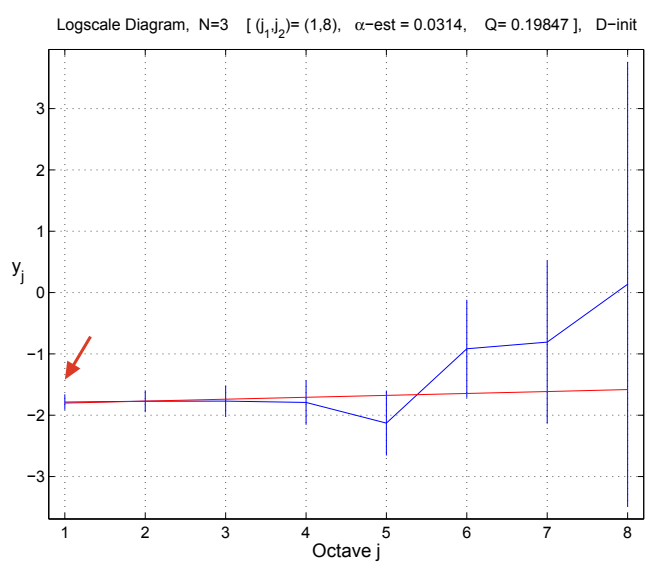

(b) Espectro wavelet $(H=0,6)$

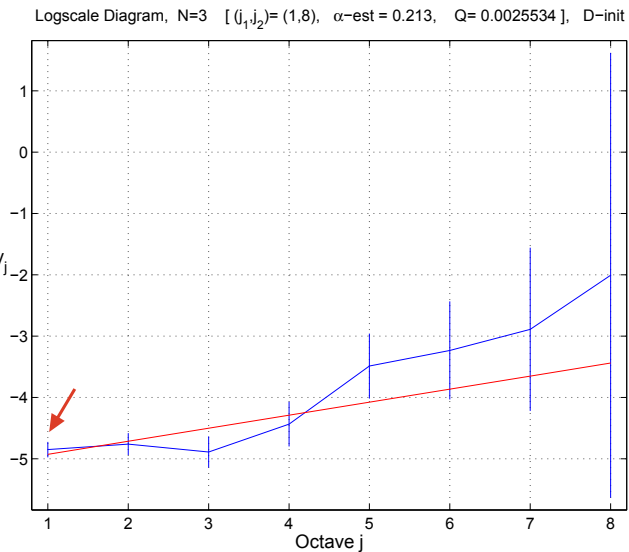

(d) Espectro wavelet $(H=0,75)$

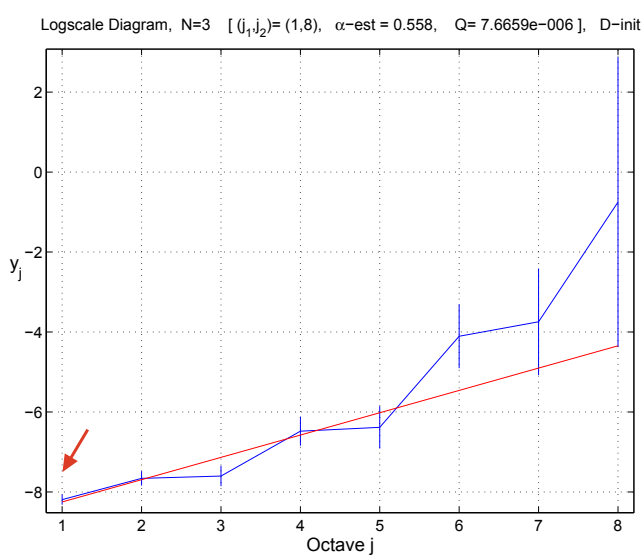

(f) Espectro wavelet $(H=0,9)$

Figura 6.5: Análise espectral das séries com $\operatorname{SRD}$ (filtragem com $f_{0} \approx 0,7$ ). 
$H)$ versus $H_{\text {gen }}$ (parâmetro $H$ utilizado na geração), as estimativas pelo método de Abry-Veitch apresentam-se com ligeiro viés positivo para valores de $H$ próximos de 0,5 , enquanto que, para valores próximos de 1,0, as estimativas de $H$ apresentam ligeiro viés negativo.

As Figuras 6.6b-d, por outro lado, demonstram que a adição de SRD pode 'perturbar' de forma não desprezível esse viés. Enquanto a Figura 6.6c demonstra que, considerando-se o filtro em questão, as estimativas, tanto de Whittle como de Abry-Veitch, não se alteraram significativamente com relação às séries sem SRD (a não ser pelas estimativas de Abry-Veitch para $H$ próximo de 1 , que apresentaram maior viés negativo do que originalmente), o mesmo não pode ser dito das Figuras 6.6b e 6.6d. A Figura 6.6b (referente à adição de SRD com o filtro com $f_{0} \approx 0,2 \pi \mathrm{rad} /$ amostra) indica que a estimativa de $H$ com Whittle mostra-se com maior viés do que a estimativa por Abry-Veitch. Além disso, as estimativas por Whittle apresentam saturação ao atingirem o valor de $H \approx 1$. Enquanto tal aspecto é fisicamente aceitável (uma vez não ser possível a geração de séries com $H>1$ ), tal não linearidade inviabiliza uma potencial correção do viés apresentado, o que não ocorre com as estimativas pelo método de AbryVeitch. Já a Figura 6.6d (referente à adição de SRD com o filtro com $f_{0} \approx$ $0,7 \pi \mathrm{rad} /$ amostra) demonstra que o viés, para ambos estimadores, é negativo e aproximadamente da mesma ordem de grandeza. 


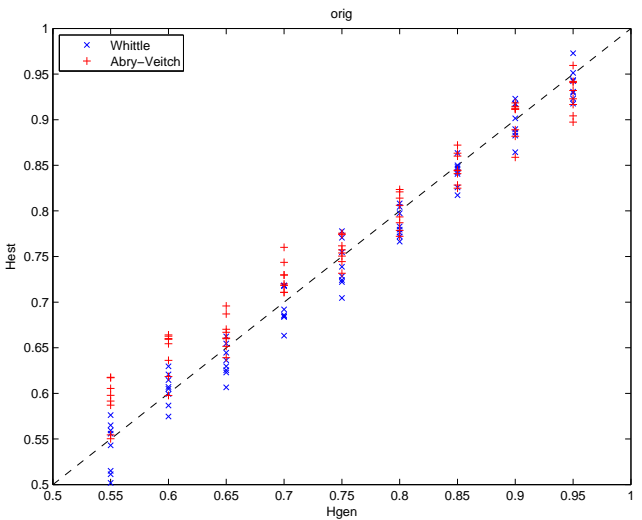

(a) Séries originais (sem SRD)

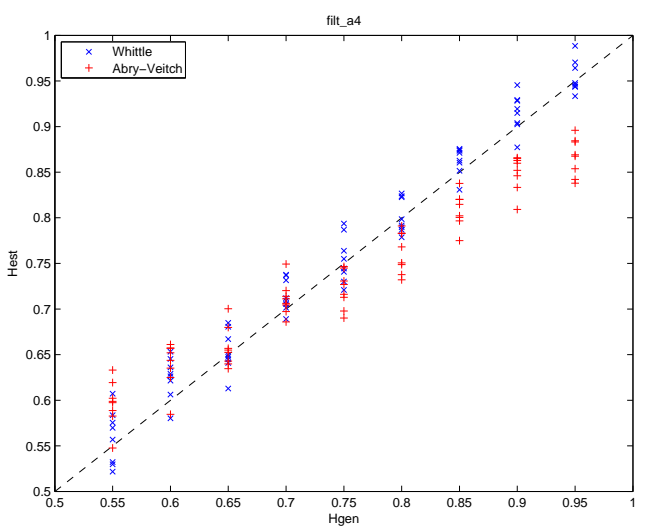

(c) Séries com $\operatorname{SRD}\left(f_{0} \approx 0,4\right)$

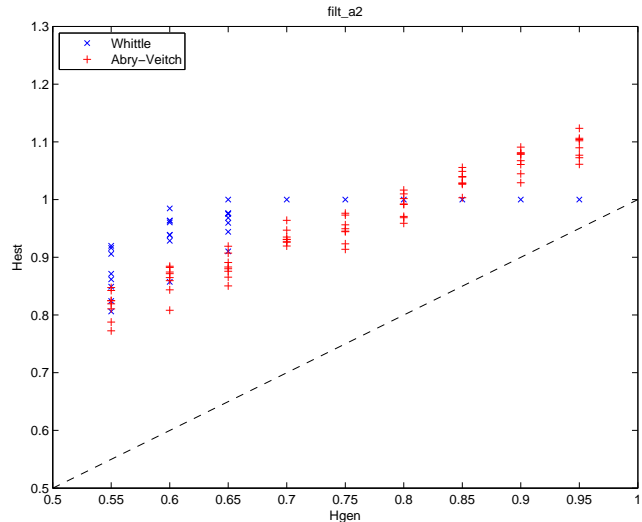

(b) Séries com $\operatorname{SRD}\left(f_{0} \approx 0,2\right)$

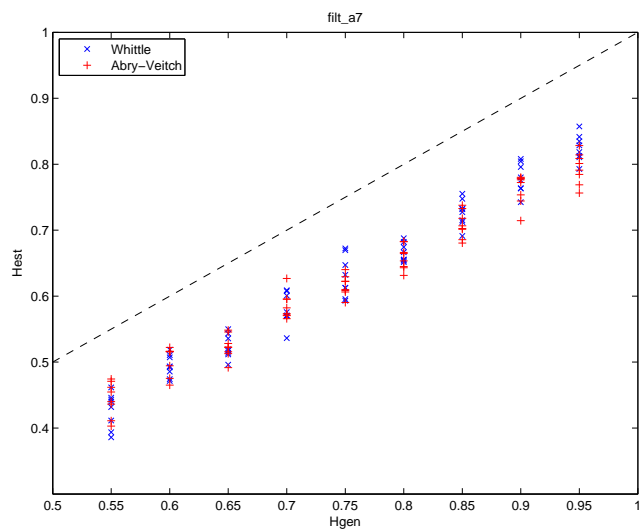

(d) Séries com $\operatorname{SRD}\left(f_{0} \approx 0,7\right)$

Figura 6.6: Efeitos da estimativa de $H$ em séries sem e com SRD. 


\subsubsection{Exploração dos resultados}

De posse dos resultados da Seção 6.1.2, iniciou-se uma séries de explorações na tentativa de reduzir o viés apresentado pelo método de estimação de Abry-Veitch na presença de SRD.

Em uma primeira tentativa, verificou-se a possibilidade de melhoria dos resultados de estimação para as séries originais (sem SRD) através da análise das estimativas para diversos valores de $j_{\min }$. Isso porque, considerando-se que as séries geradas apresentam LRD mas não são exatamente $H$-ss, o comportamento LRD não será apresentado em todas as escalas wavelets, mas apenas nas escalas mais lentas (ou seja, para maiores valores de $j$ ).

A Figura 6.7 apresenta ao resultados obtidos, indicando, para cada valor de $H$ utilizado na geração das séries, o histograma e o Quantile-Quantile plot (QQplot) do conjunto de estimativas de $H$ para $j_{\max }=8$ e $1 \leq j_{\min } \leq 6$. Tais gráficos demonstram que as estimativas seguem uma distribuição aproximadamente normal para valores próximos da média, comportamento este não observado nas extremidades da distribuição, que apresenta um número considerável de pontos fora da distribuição, os chamados outliers. Já a Figura 6.8 apresenta o gráfico de $\hat{H}$ em função de $j_{\text {min }}$, para cada uma das 8 realizações geradas para cada valor de $H$. Tal gráfico (que apresenta a média, moda e mediana de tais estimativas) indica que as medidas de tendência central analisadas apresentam alguns pontos de cruzamento com a reta que indica o valor esperado de $\hat{H}$, o que pode ser uma indicação de que uma escolha criteriosa do valor de $j_{\min }$ utilizado na estimação pode eventualmente corrigir o viés observado.

De forma análoga às Figuras 6.7-6.8, as Figuras 6.9-6.10 apresentam os resultados para o caso de inclusão de SRD com o filtro de frequência central $f_{0} \approx 0,2 \pi \mathrm{rad} /$ amostra, as Figuras 6.11-6.12 apresentam os resultados para o caso de inclusão de SRD com o filtro de frequência central $f_{0} \approx 0,4 \pi \mathrm{rad} /$ amostra e as Figuras 6.13-6.14 apresentam os resultados para o caso de inclusão de SRD com o filtro de frequência central $f_{0} \approx 0,7 \pi \mathrm{rad} /$ amostra.

Ainda considerando-se a variação de $j_{\min }$ entre todos seus possíveis valores, a Figura 6.15 apresenta as estimativas para cada realização e valor de $H_{\text {gen }}$ das séries geradas. A Figura 6.15 apresenta, ainda, as curvas correspondentes às medidas de tendência central (média, moda e mediana) observadas para cada valor de $H_{g e n}$. Embora, pela inspeção visual, não se possa afirmar quais das medidas apresentam menor viés, pode-se afirmar que a moda e a mediana apresentaram dispersão 
menor do que a média, indicando que talvez possam ser melhores estimadores de $H$ do que a média.

Além disso, considerando-se a existência de número considerável de outliers quando é estimado o valor de $H$ baseando-se em todas as possíveis estimativas para os diversos valores de $j_{\text {min }}$, foram removidos os outliers de tais estimativas e gerado novo gráfico análogo à Figura 6.15. O resultado desse estudo é apresentado na Figura 6.16 que, para melhor clareza, apresenta apenas o resultado da média das estimativas. A Figura 6.16 evidencia que a remoção de tais outliers, embora possa contribuir para a redução da variabilidade das estimativas, introduz um notório viés nas mesmas, não se mostrando uma boa alternativa a ser adotada.

Para concluir a análise preliminar, realizou-se a sobreposição dos espectros wavelet obtidos pelas estimativas por Abry-Veitch para os casos de séries sem SRD e para as séries com a adição de SRD. Os resultados estão na Figura 6.17, onde a linha contínua representa o espectro wavelet, e a linha tracejada representa a respectiva regressão linear realizada. Conforme evidenciado pelas Figuras 6.17ab, para valores de $H \leq 0,75$, os espectros wavelet da série original e das séries com SRD são, conforme inspeção visual, bastante semelhantes para $j \geq 5$, sugerindo que, caso a estimação de $H$ seja realizada considerando-se $j_{\min }=5$, as estimativas obtidas independeriam da presença ou não de SRD. Para a Figura 6.17c, embora os espectros das séries com SRD sejam muito semelhantes para $j \geq 5$ (como nos casos anteriores), não se pode concluir com a inspeção visual que tais espectros são paralelos ao espectro da série original. Isso, entretanto, não necessariamente invalida a suposição de que as retas ajustadas a tais espectros tenham a mesma declividade (e, portanto, resultem na mesma estimativa de $H$ ). 


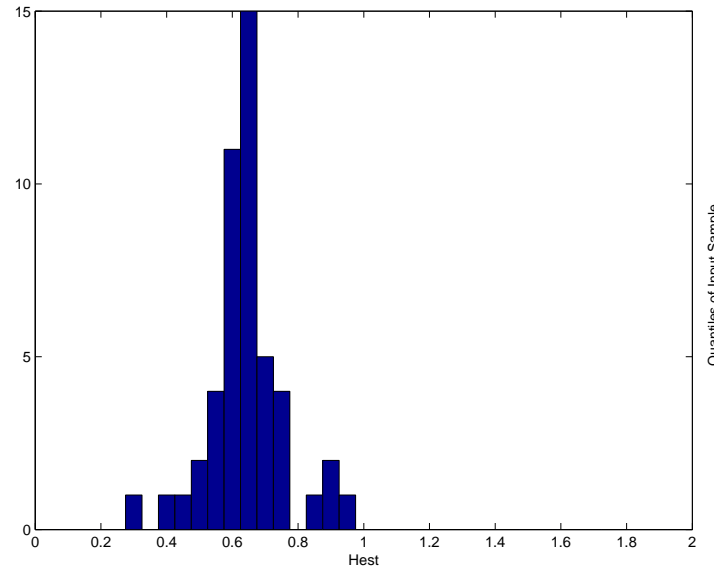

(a) Histograma $(H=0,6)$

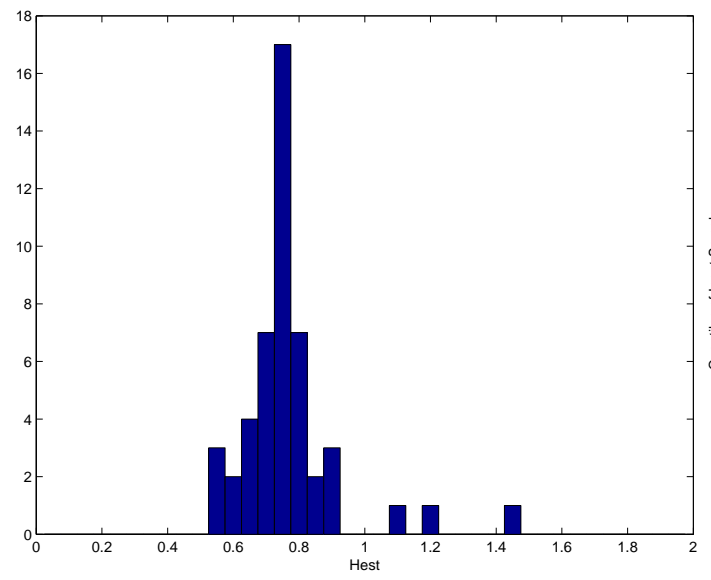

(c) Histograma $(H=0,75)$

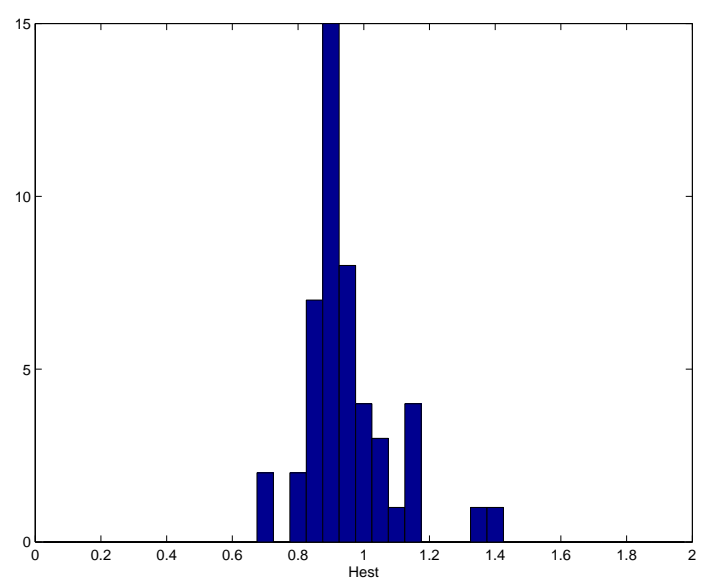

(e) Histograma $(H=0,9)$

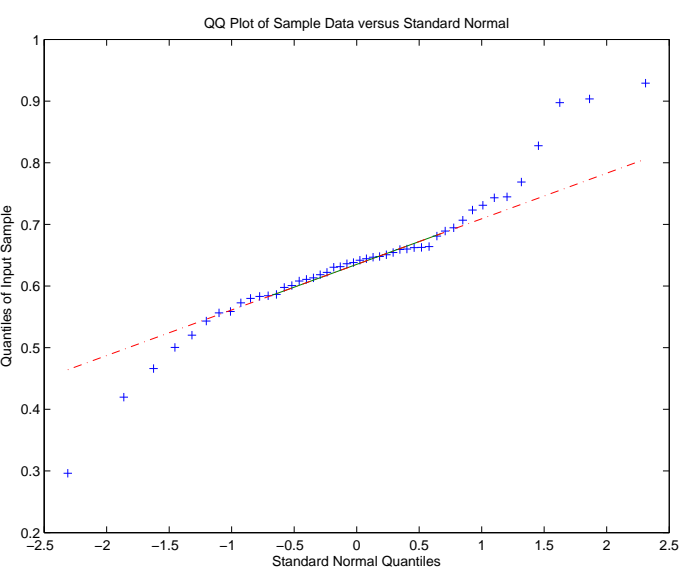

(b) QQ-Plot $(H=0,6)$

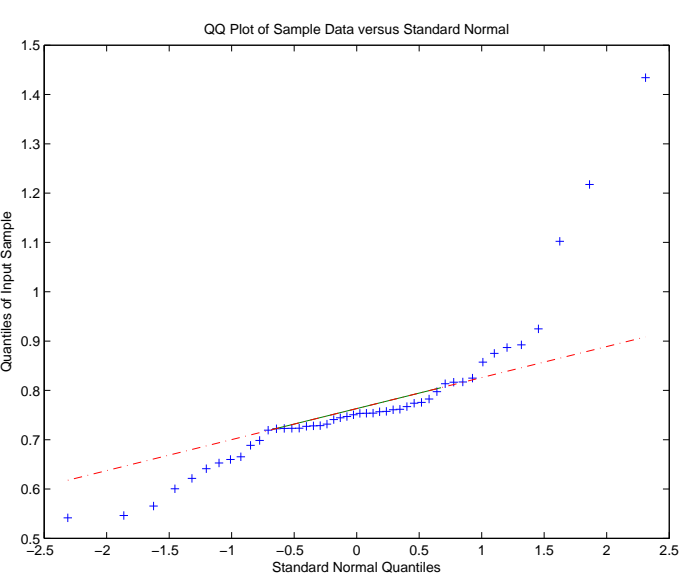

(d) QQ-Plot $(H=0,75)$

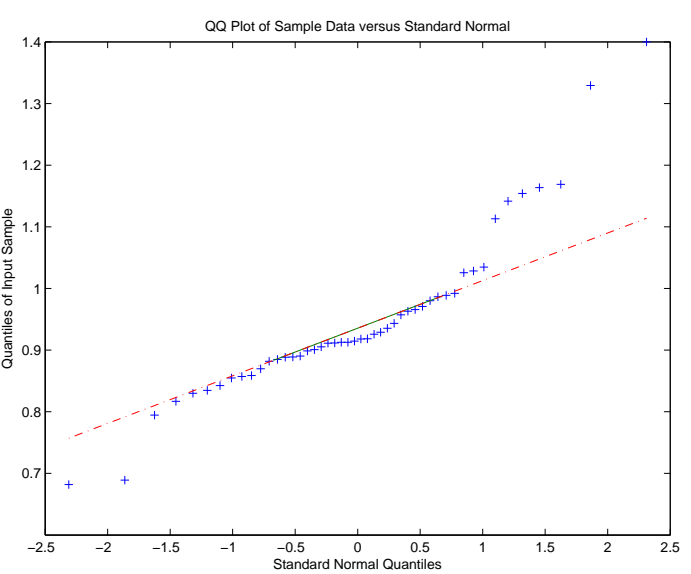

(f) QQ-Plot $(H=0,9)$

Figura 6.7: Histograma e QQ-plot $\hat{H}$ para diversos valores de $j_{\min }$ (séries originais sem $\mathrm{SRD}$ ). 


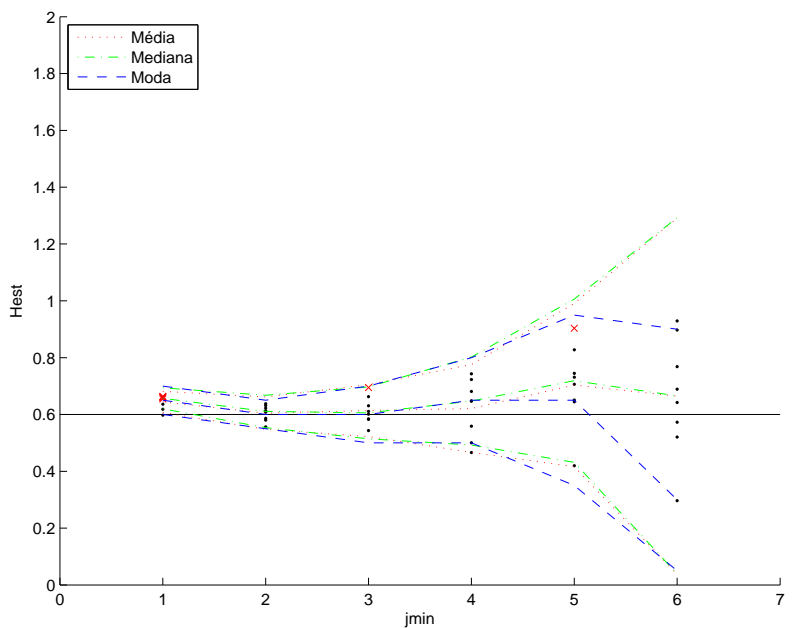

(a) $H_{\text {est }}$ vs. $j_{\min }(H=0,6)$

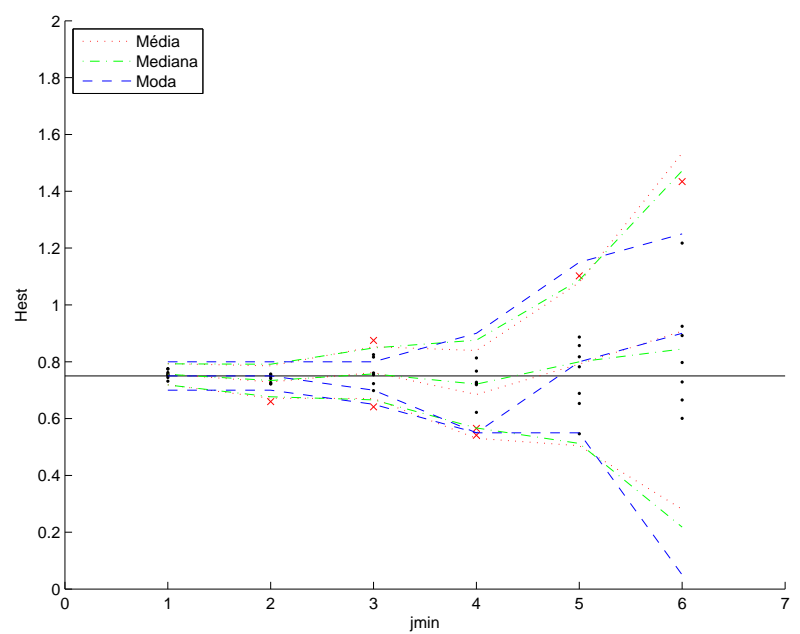

(b) $H_{\text {est }}$ vs. $j_{\min }(H=0,75)$

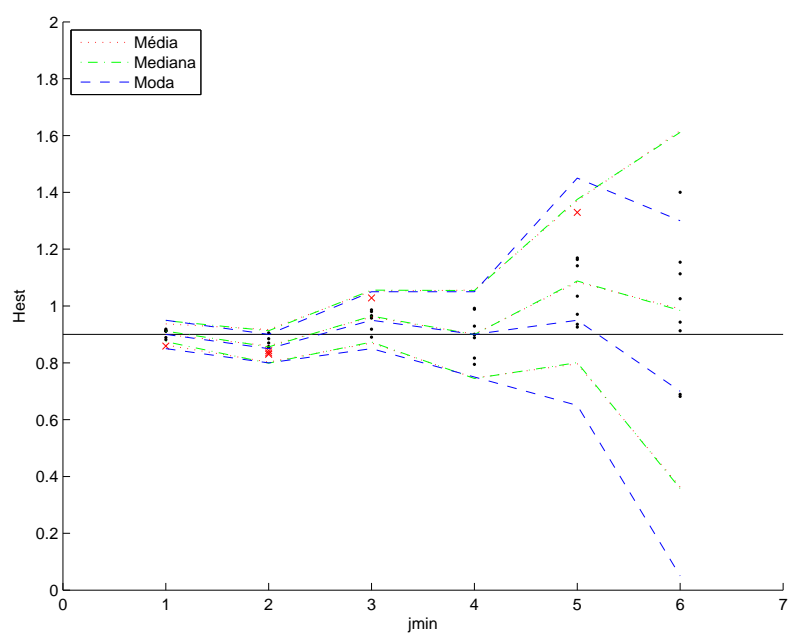

(c) $H_{\text {est }}$ vs. $j_{\min }(H=0,9)$

Figura 6.8: Estatísticas de $\hat{H}$ para diversos valores de $j_{\text {min }}$ (séries originais sem SRD). 


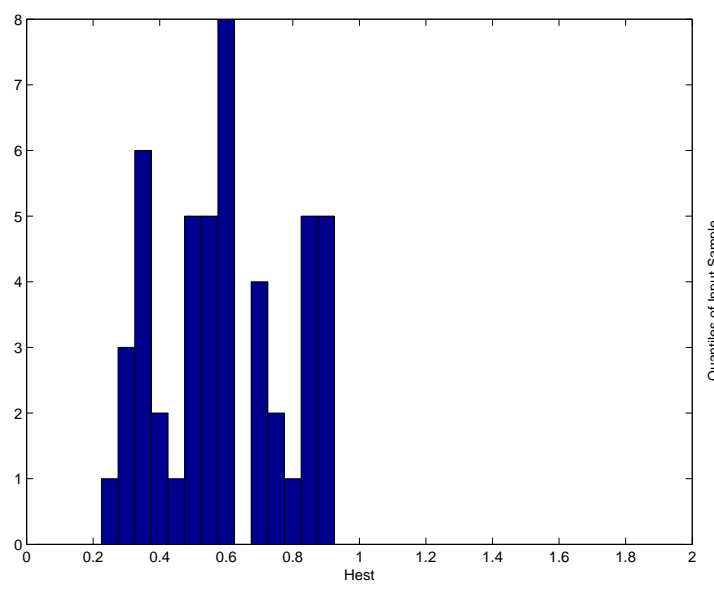

(a) Histograma $(H=0,6)$

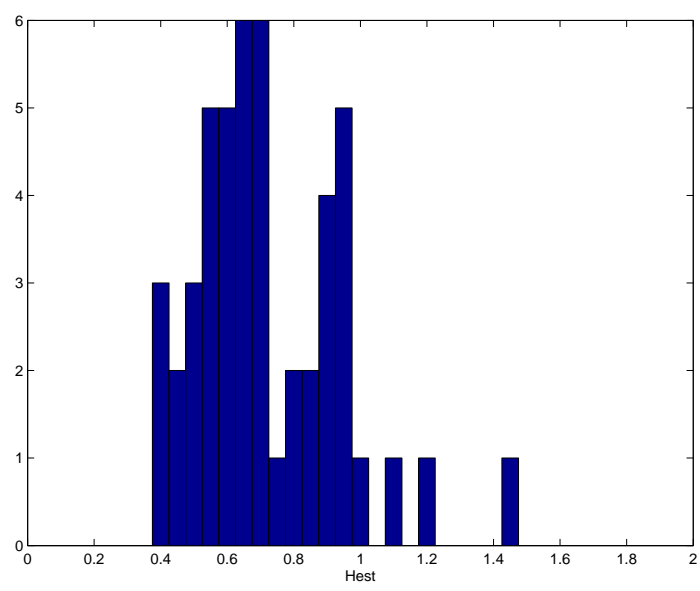

(c) Histograma $(H=0,75)$

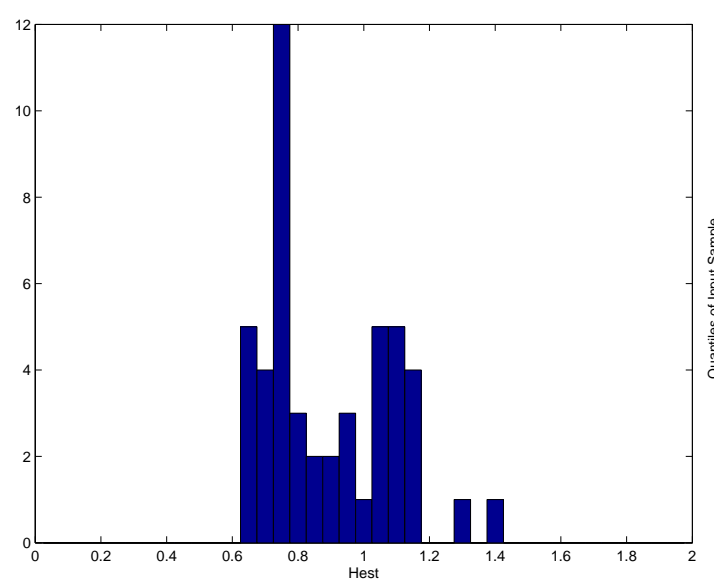

(e) Histograma $(H=0,9)$

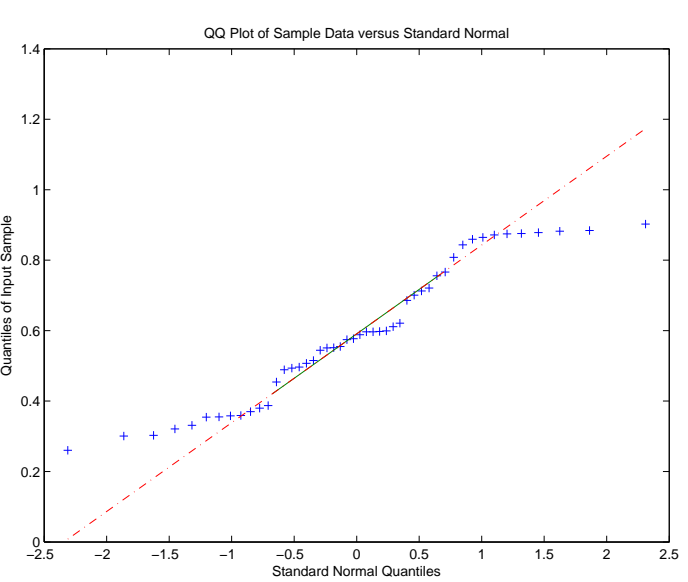

(b) QQ-Plot $(H=0,6)$

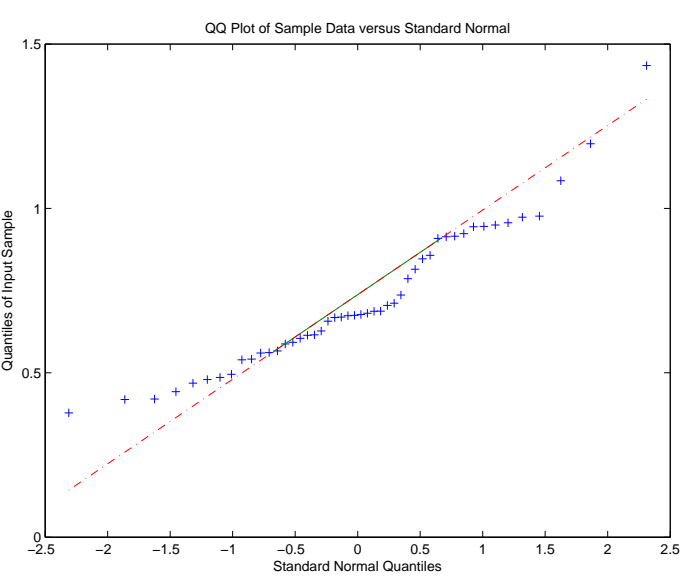

(d) QQ-Plot $(H=0,75)$

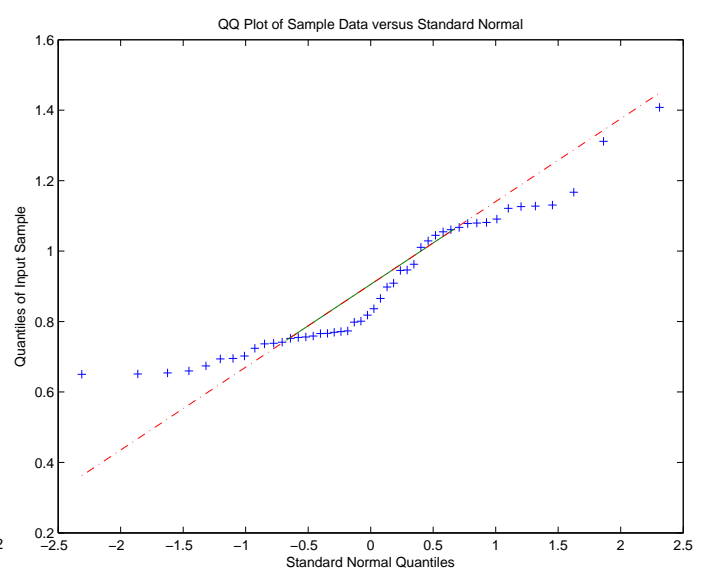

(f) QQ-Plot $(H=0,9)$

Figura 6.9: Histograma e QQ-plot para diversos valores de $j_{\min }$ (séries com SRD - $f_{0} \approx 0,2 \pi \mathrm{rad} /$ amostra). 


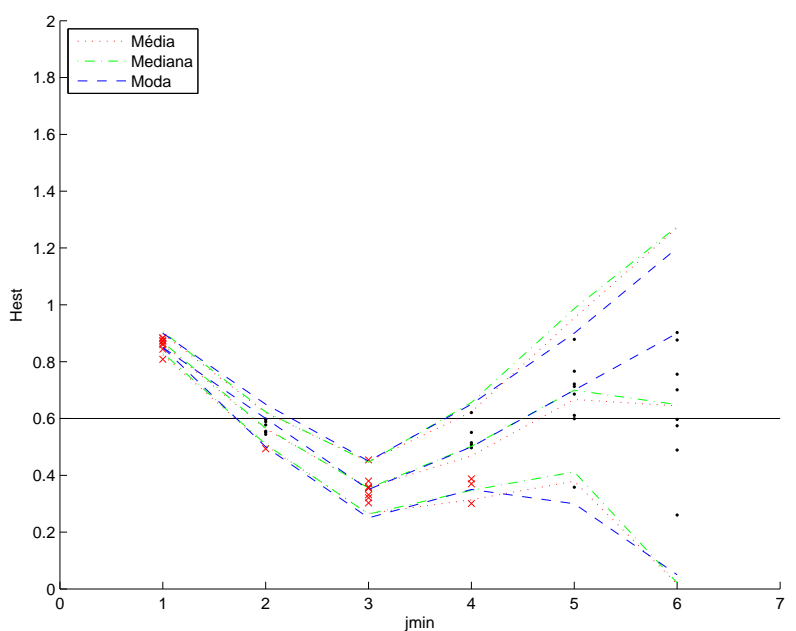

(a) $H_{e s t}$ vs. $j_{\min }(H=0,6)$

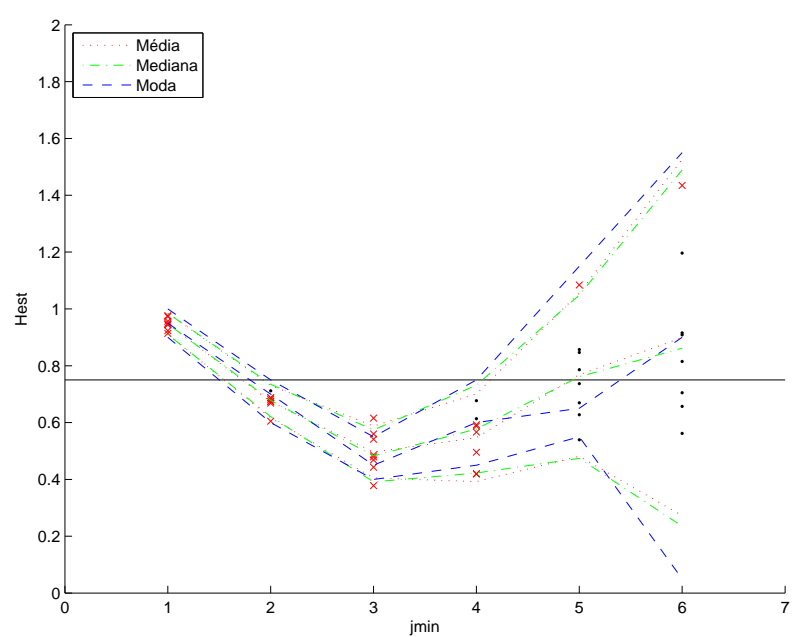

(b) $H_{e s t}$ vs. $j_{\min }(H=0,75)$

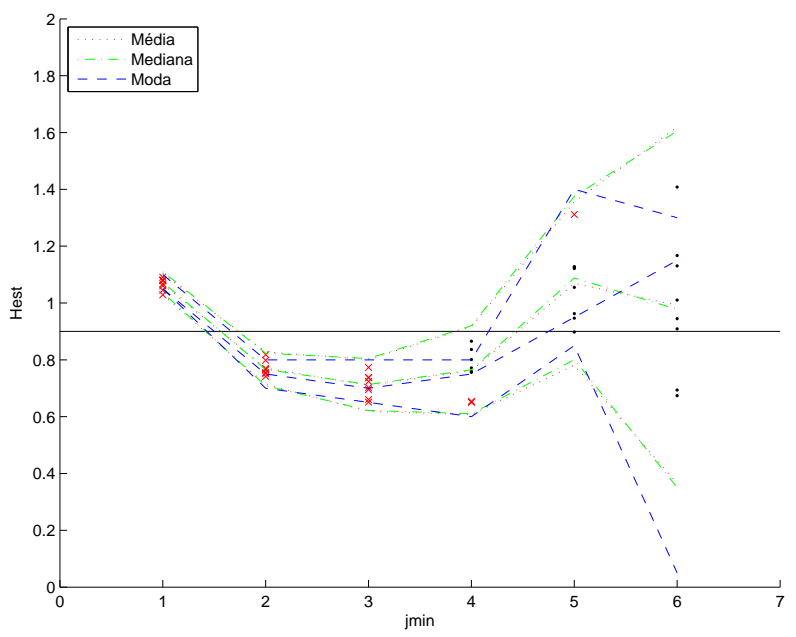

(c) $H_{\text {est }}$ vs. $j_{\min }(H=0,9)$

Figura 6.10: Estatísticas de $\hat{H}$ para diversos valores de $j_{\text {min }}$ (séries com SRD - $f_{0} \approx 0,2 \pi \mathrm{rad} /$ amostra). 


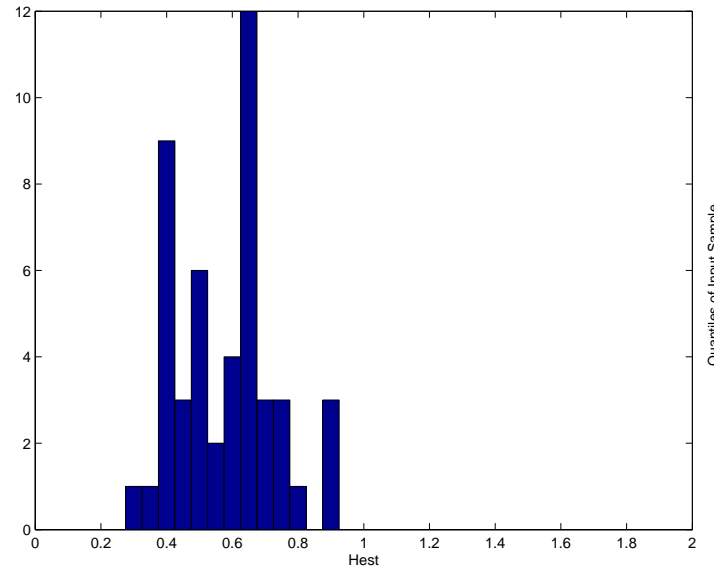

(a) Histograma $(H=0,6)$

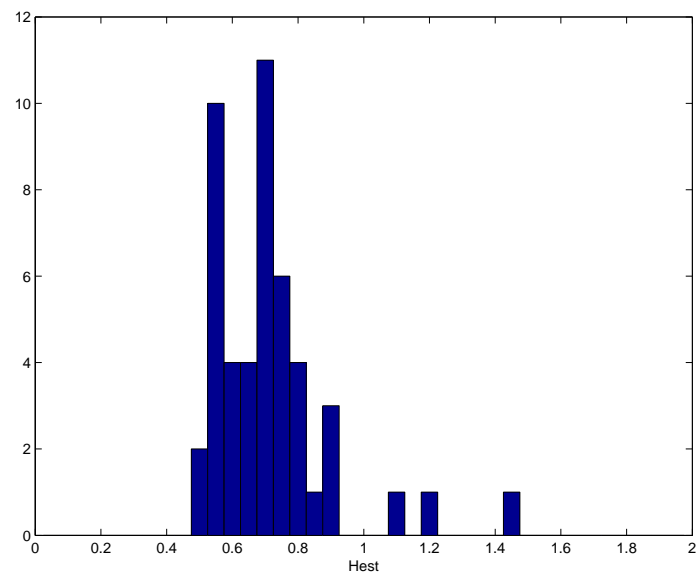

(c) Histograma $(H=0,75)$

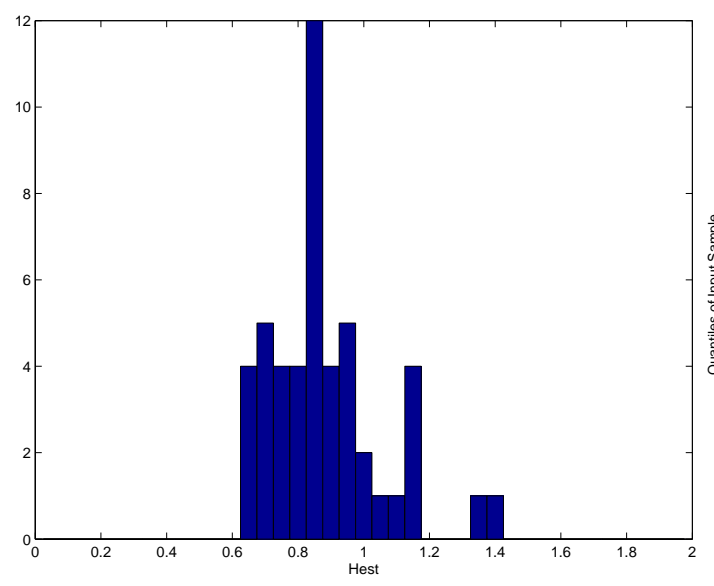

(e) Histograma $(H=0,9)$

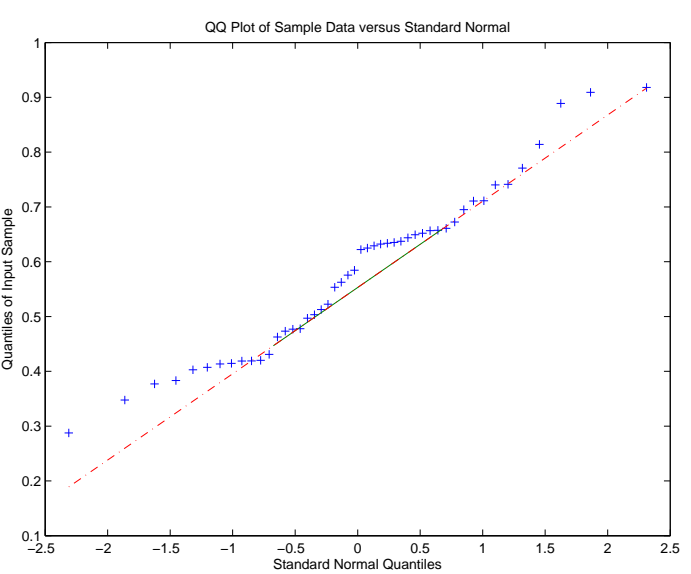

(b) QQ-Plot $(H=0,6)$

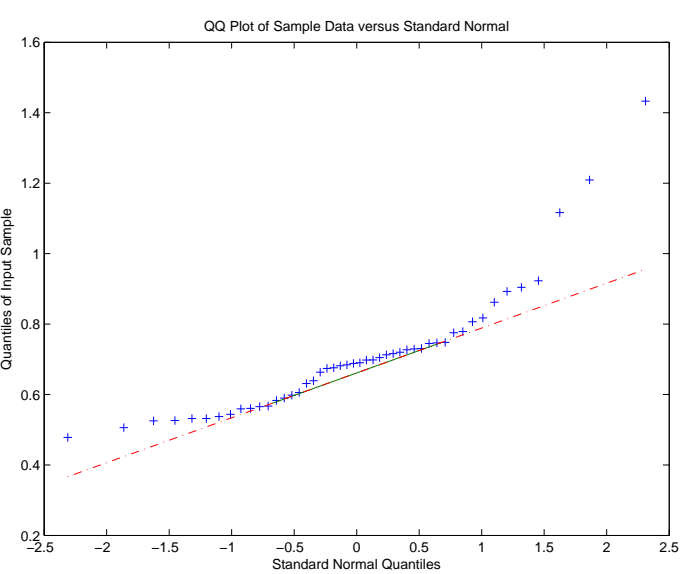

(d) QQ-Plot $(H=0,75)$

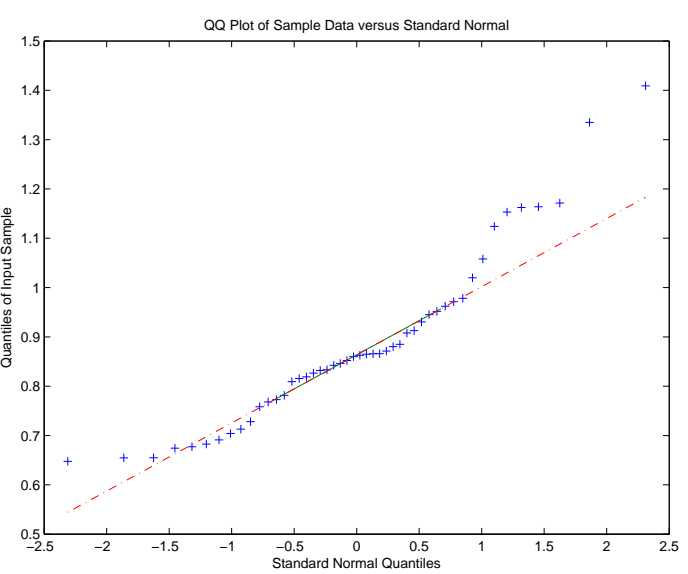

(f) QQ-Plot $(H=0,9)$

Figura 6.11: Histograma e QQ-plot para diversos valores de $j_{\text {min }}$ (séries com SRD - $f_{0} \approx 0,4 \pi \mathrm{rad} /$ amostra). 


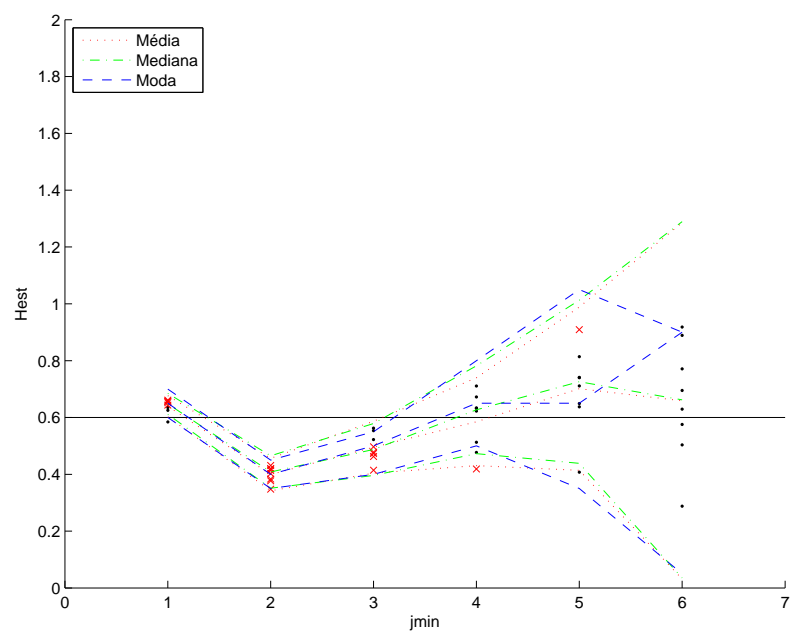

(a) $H_{e s t}$ vs. $j_{\min }(H=0,6)$

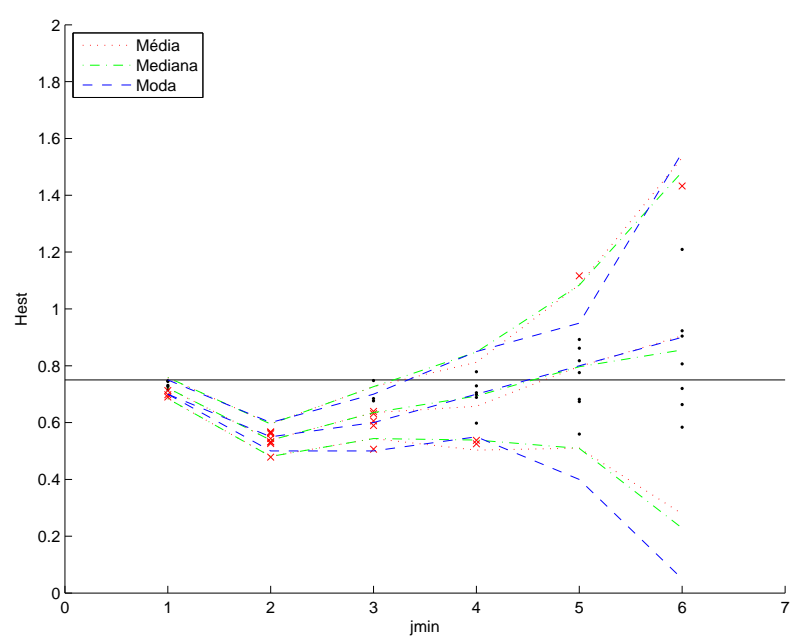

(b) $H_{e s t}$ vs. $j_{\min }(H=0,75)$

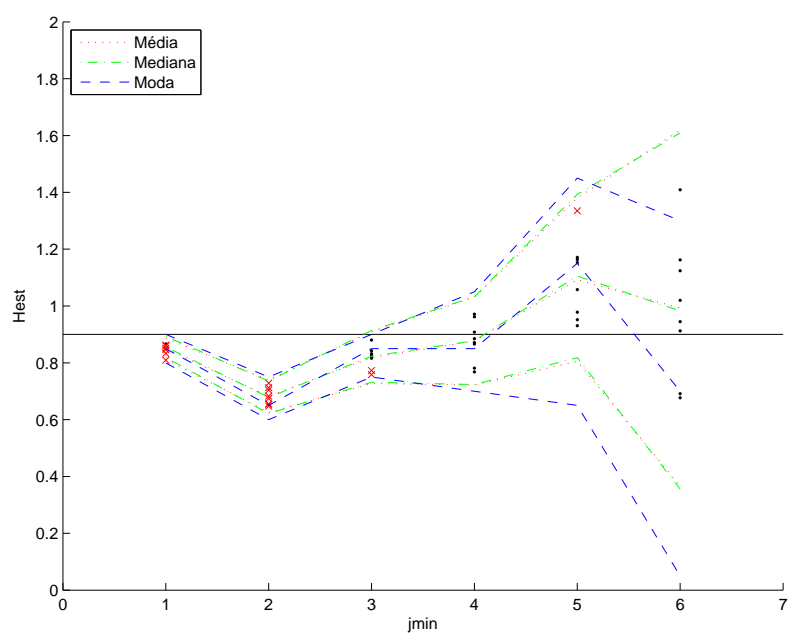

(c) $H_{\text {est }}$ vs. $j_{\min }(H=0,9)$

Figura 6.12: Estatísticas de $\hat{H}$ para diversos valores de $j_{\text {min }}$ (séries com SRD - $f_{0} \approx 0,4 \pi \mathrm{rad} /$ amostra). 


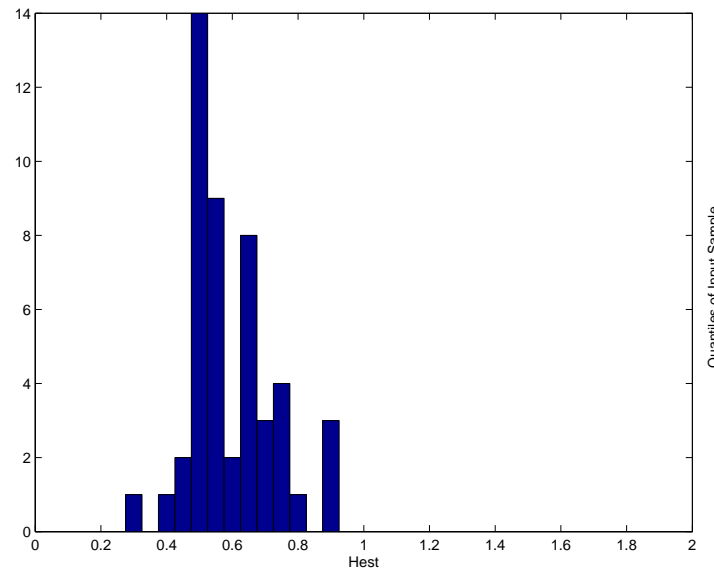

(a) Histograma $(H=0,6)$

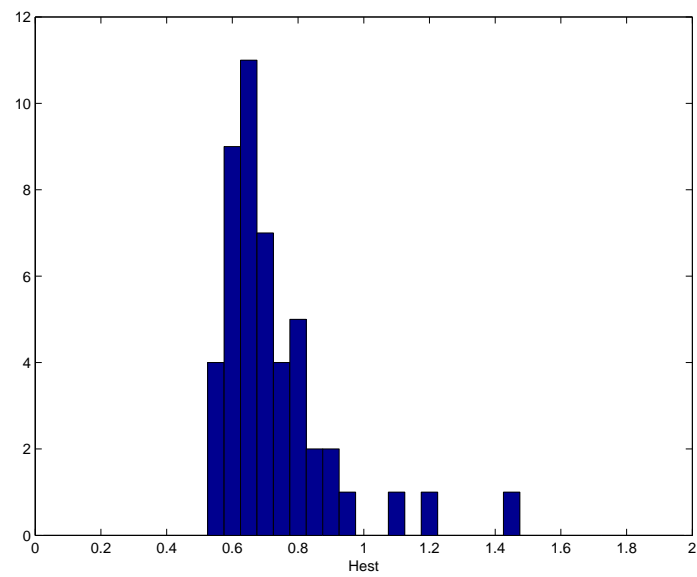

(c) Histograma $(H=0,75)$

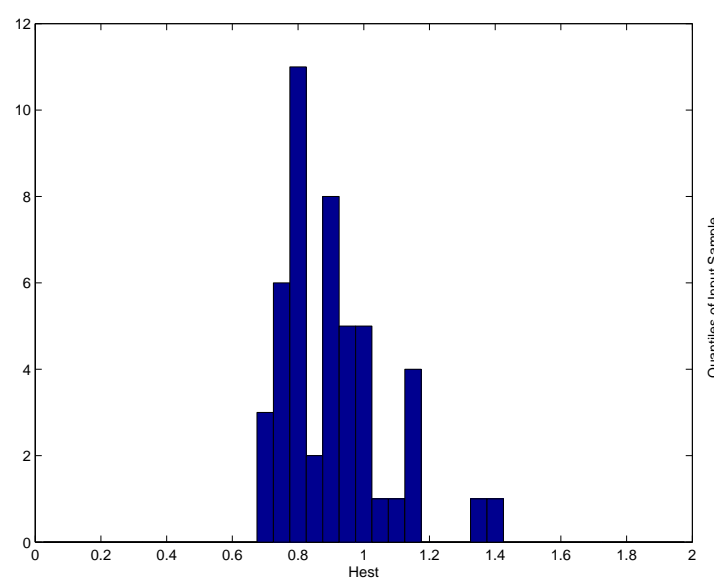

(e) Histograma $(H=0,9)$

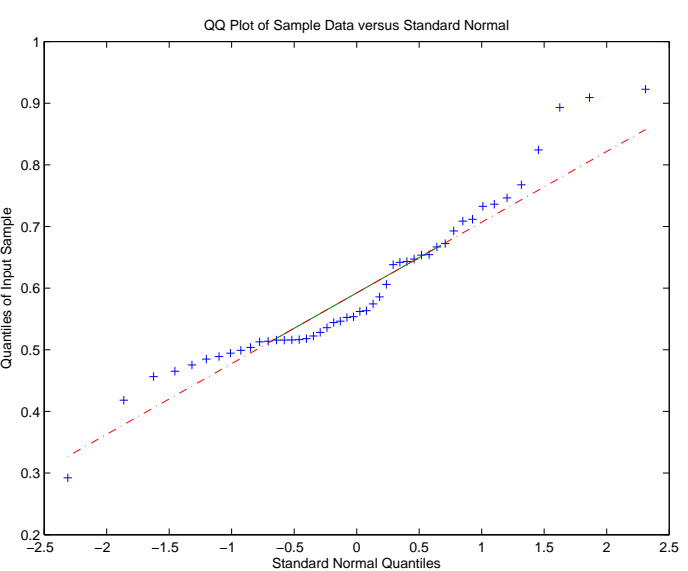

(b) QQ-Plot $(H=0,6)$

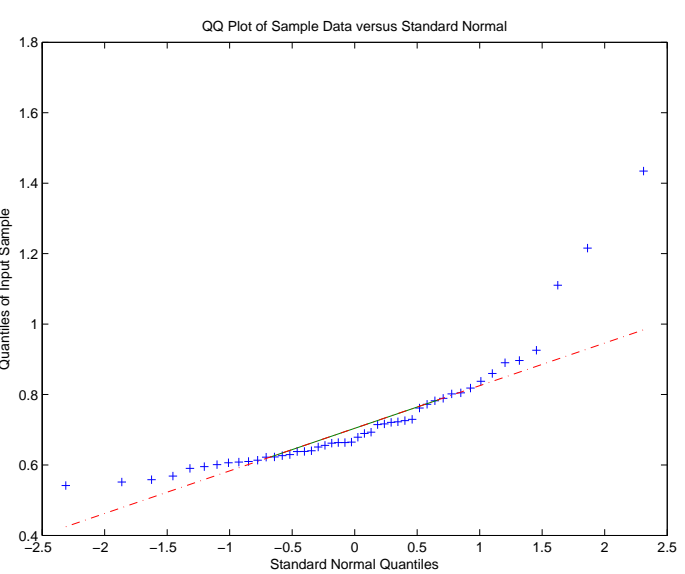

(d) QQ-Plot $(H=0,75)$

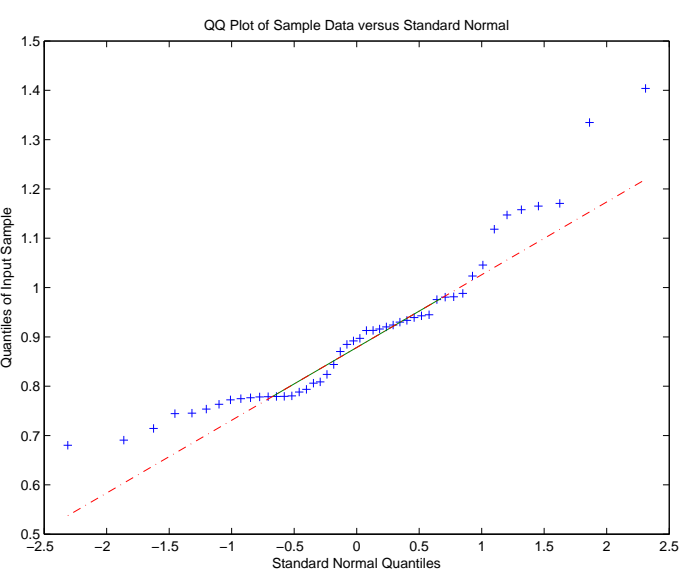

(f) QQ-Plot $(H=0,9)$

Figura 6.13: Histograma e QQ-plot para diversos valores de $j_{\text {min }}$ (séries com SRD - $f_{0} \approx 0,7 \pi \mathrm{rad} /$ amostra). 


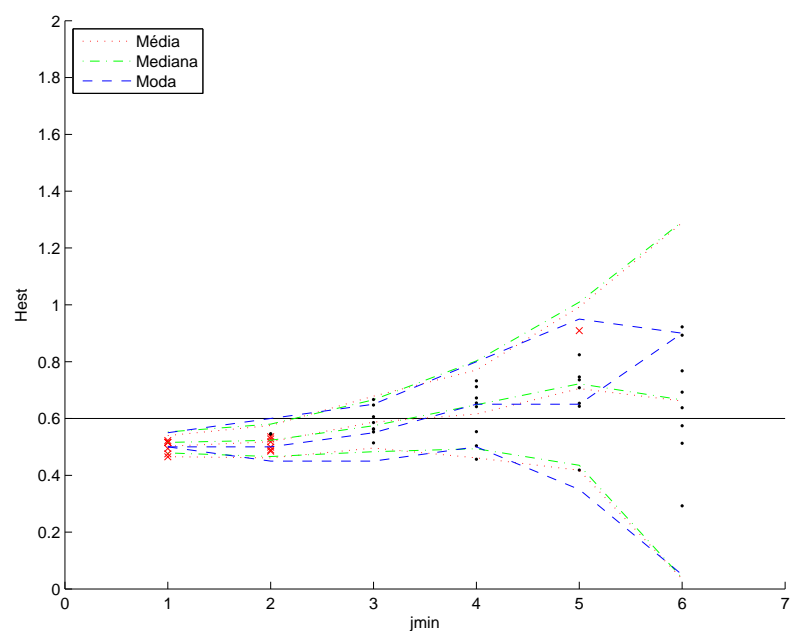

(a) $H_{e s t}$ vs. $j_{\min }(H=0,6)$

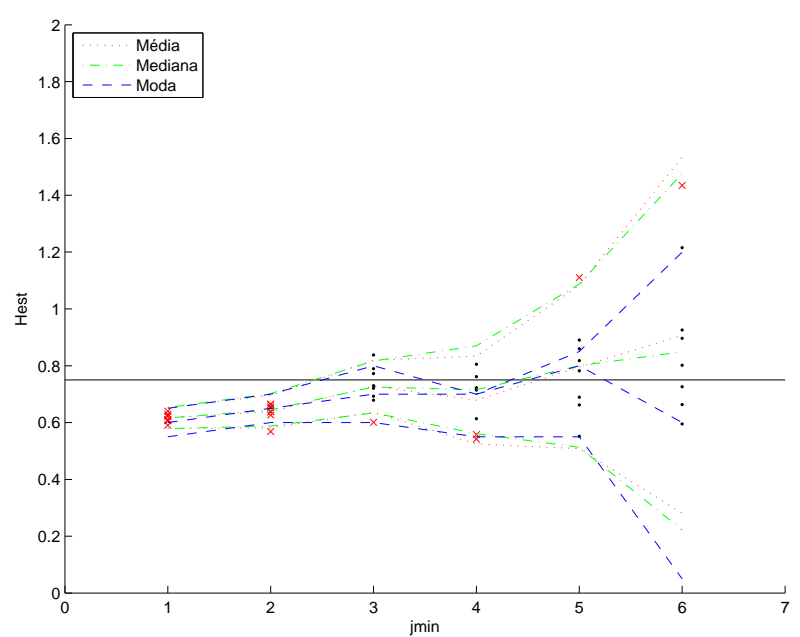

(b) $H_{e s t}$ vs. $j_{\min }(H=0,75)$

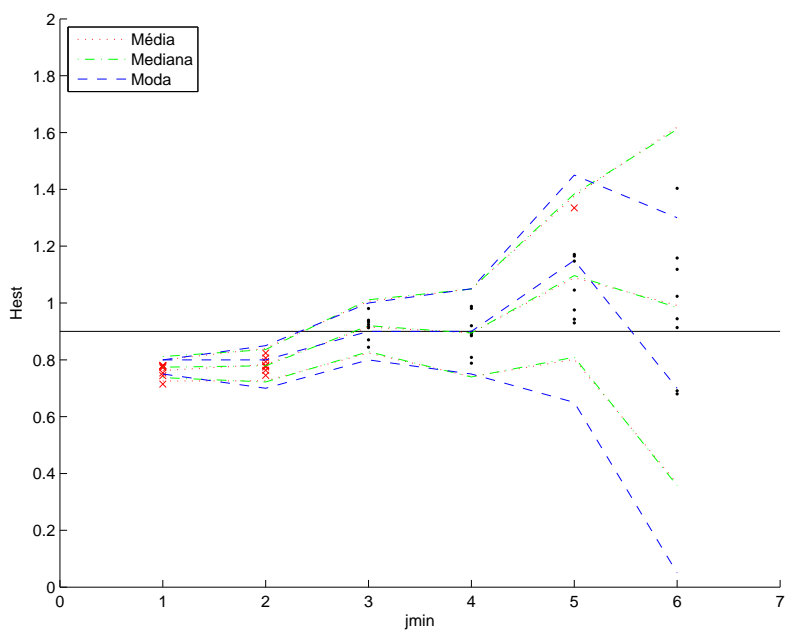

(c) $H_{\text {est }}$ vs. $j_{\min }(H=0,9)$

Figura 6.14: Estatísticas de $\hat{H}$ para diversos valores de $j_{\text {min }}$ (séries com $\mathrm{SRD}-f_{0} \approx 0,7 \pi \mathrm{rad} /$ amostra). 


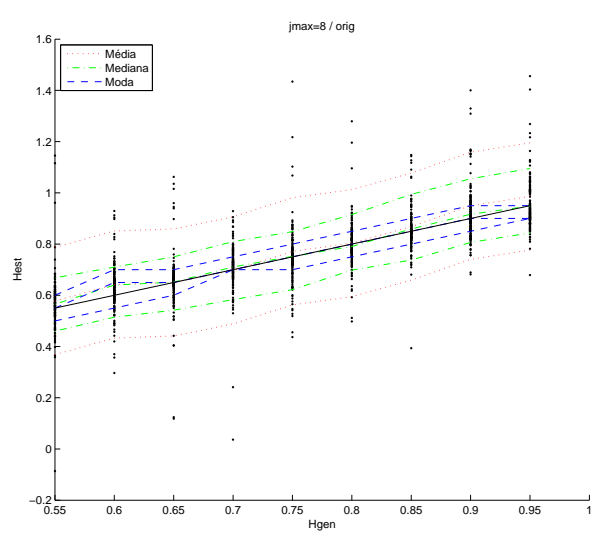

(a) Séries originais sem SRD

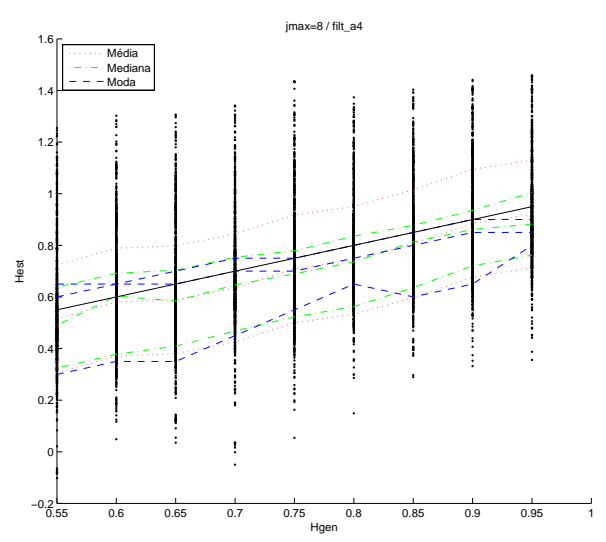

(c) Séries com SRD - $f_{0} \approx 0,4$

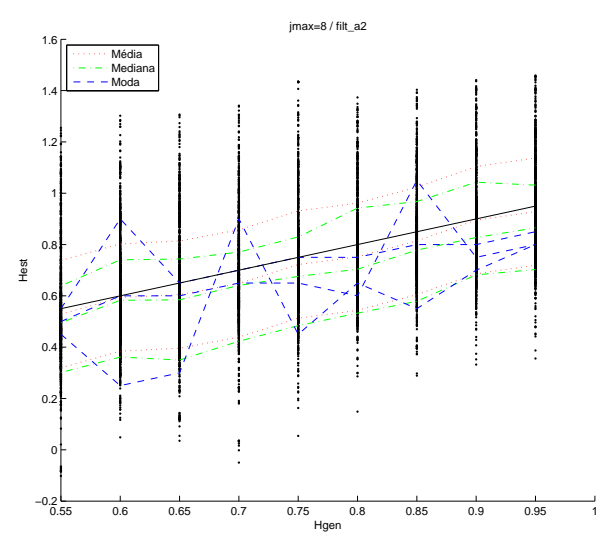

(b) Séries com SRD - $f_{0} \approx 0,2$

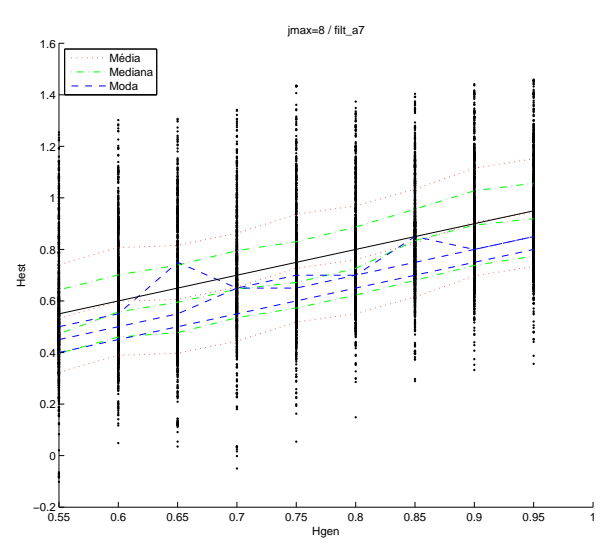

(d) Séries com SRD - $f_{0} \approx 0,7$

Figura 6.15: Estimativas considerando-se todos os possíveis valores de $j_{\text {min }}$. 


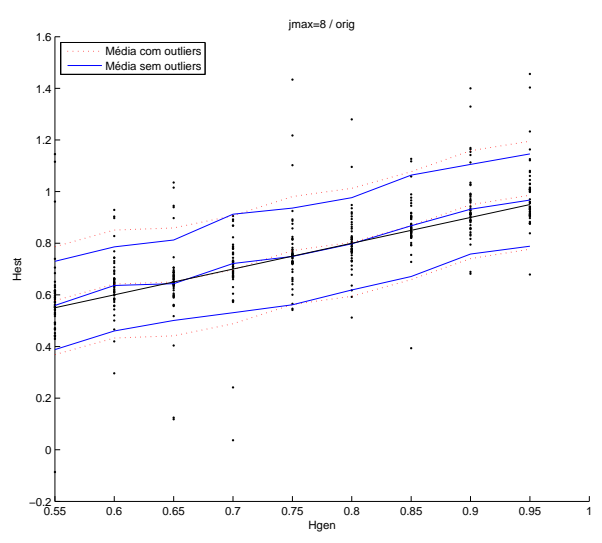

(a) Séries originais sem SRD

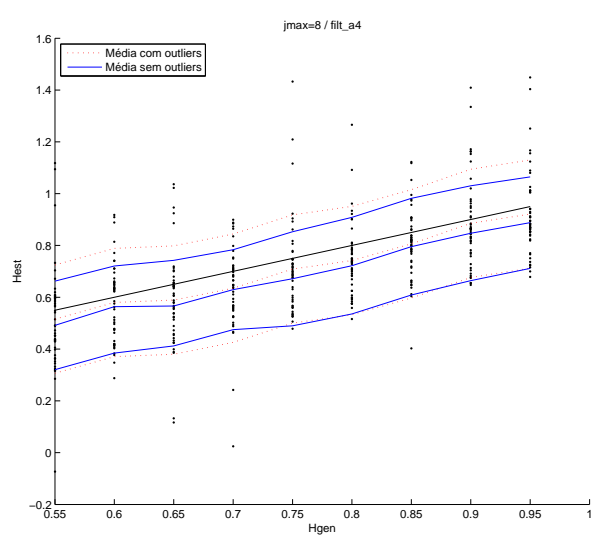

(c) Séries com SRD - $f_{0} \approx 0,4$

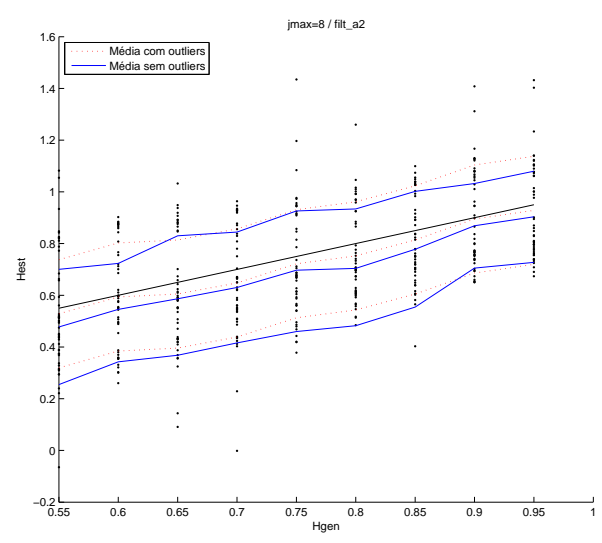

(b) Séries com SRD - $f_{0} \approx 0,2$

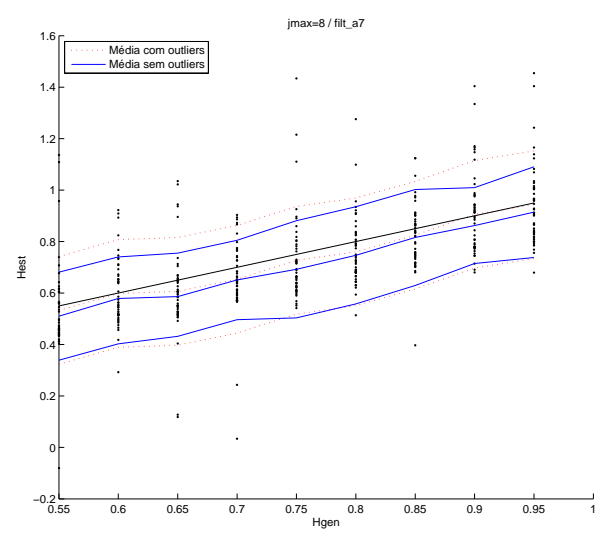

(d) Séries com SRD - $f_{0} \approx 0,7$

Figura 6.16: Estimativas considerando-se todos os possíveis valores de $j_{\text {min }}$ excluindo-se os outliers. 


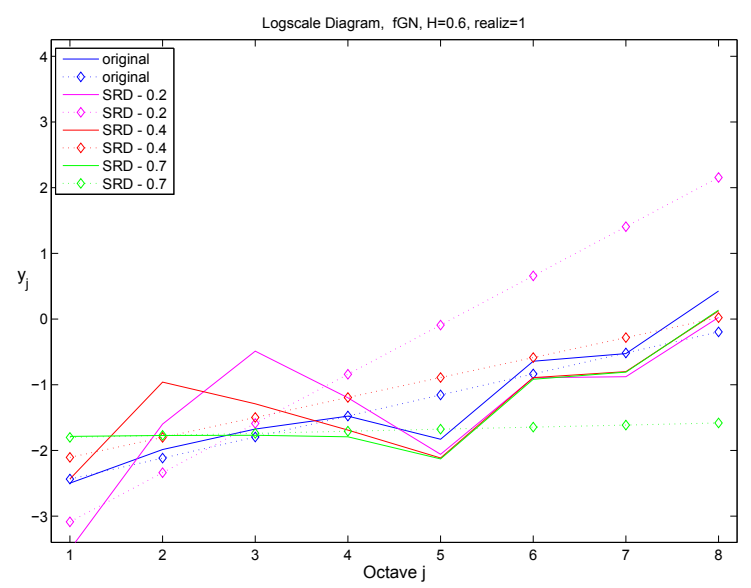

(a) $H=0,6$

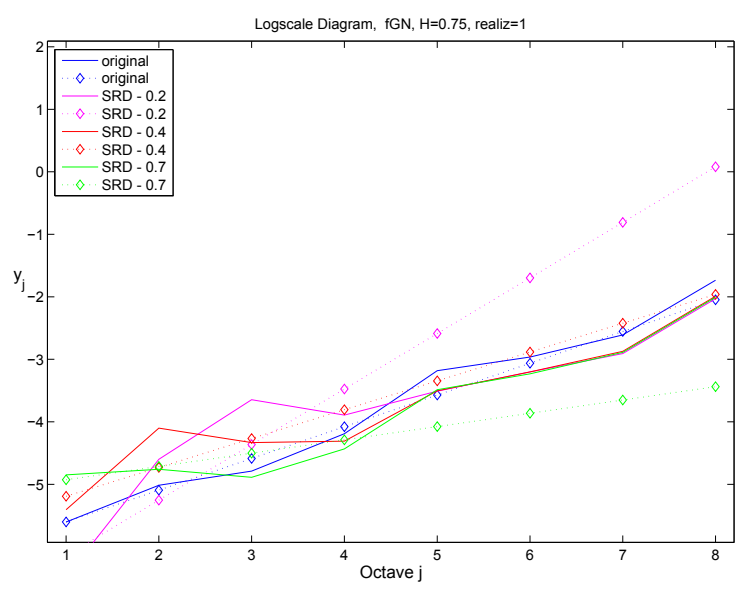

(b) $H=0,75$

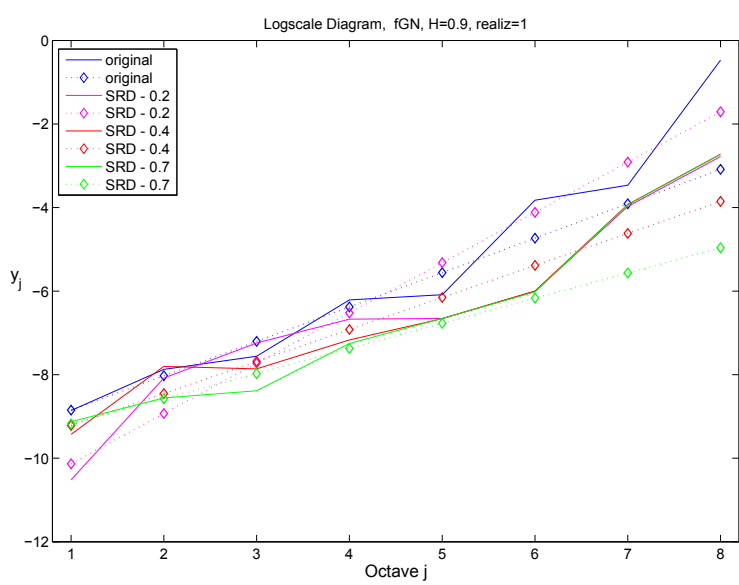

(c) $H=0,9$

Figura 6.17: Comparação do espectro wavelet entre séries sem e com SRD. 


\subsubsection{Síntese dos resultados}

A Tabela 6.1 apresenta uma síntese dos principais resultados expostos na Seção 6.1 .

Tabela 6.1: Resultados da Seção 6.1.

\begin{tabular}{|c|l|}
\hline \hline Resultado & Descrição \\
\hline \hline 1 & $\begin{array}{l}\text { Demonstração da presença de viés na estimação de } H \text { quando da presença de } \\
\text { SRD }\end{array}$ \\
\hline 2 & $\begin{array}{l}\text { Comparação das estimativas de } H \text {, na presença de SRD, para os estimadores } \\
\text { de Whittle e de Abry-Veitch: viés bastante notório em ambos estimadores }\end{array}$ \\
\hline 3 & $\begin{array}{l}\text { Observação do efeito da variação do parâmetro } j_{m i n} \text { na estimação de } H: \text { au- } \\
\text { mento do valor de } j_{m i n} \text { pode corrigir viés identificado na presença de SRD às } \\
\text { custas de um aumento na dispersão das estimativas }\end{array}$ \\
\hline 4 & $\begin{array}{l}\text { Identificação de comportamento não normal na cauda da distribuição das esti- } \\
\text { mativas considerando-se todos os possíveis } j_{m i n} \text { : tentativa de redução do viés } \\
\text { desconsiderando-se outliers mostrou-se pouco frutífera }\end{array}$ \\
\hline 5 & $\begin{array}{l}\text { Identificação nos gráficos do espectro wavelet de presença de possível } j_{\min } \text { ca- } \\
\text { racterístico tal que estimativa de } H \text { independeria da presença de SRD }\end{array}$ \\
\hline \hline
\end{tabular}




\subsection{Análise da influência dos parâmetros de es- timação do Método de Abry-Veitch}

Os resultados da Seção 6.1 demonstraram o efeito potencial da variação do parâmetro $j_{\min }$ na estimação de $H$ na presença de SRD. De posse desse resultado, a presente Seção explora em profundidade os efeitos da variação tanto da escala mínima do espectro wavelet (parâmetro $j_{\text {min }}$ ) como do número de amostras utilizadas para estimação (parâmetro $N$ ) na qualidade da estimação do parâmetro $H$ na presença de SRD. Enquanto a escolha apropriada do parâmetro $j_{\text {min }}$, conforme demonstrado, pode eventualmente anular o efeito potencialmente negativo da componente SRD na estimação de $H$, a escolha apropriada do número de amostras utilizadas na estimação é um importante fator na estimação em tempo real de $H$, conforme será demonstrado em seguida.

\subsubsection{Condições experimentais}

As mesmas condições experimentais apresentadas na Seção 6.1.1 foram consideradas para os experimentos apresentados a seguir, tanto com relação à geração das séries utilizadas, como com relação às estimações realizadas. A única exceção diz respeito ao número de amostras por realização: ao invés de 2048 amostras, cada realização foi gerada com $65536\left(2^{16}\right)$ amostras. Esse aumento no número de amostras mostrou-se necessário para que, para uma mesma realização, fosse possível realizar estimações de $H$ com valores de $N$ de diferentes ordens de grandeza.

Os resultados apresentados na Seção 6.2.2 levam em consideração as seguintes condições:

- $N=2^{n}$, onde $n=8,10, \ldots, 14,16$

- $j_{\min } \in\left\{1, j_{\min }^{E Q M}\right\}$

\subsubsection{Resultados obtidos}

Com o objetivo de verificar a influência da escolha de $j_{\min }$ na qualidade das estimativas de $H$ pelo método de Abry-Veitch, foram analisadas as estimativas obtidas das séries obtidas conforme detalhado na Seção 6.2.1. Tais estimativas foram separadas em grupos conforme o número de amostras da série analisada $(N)$, presença ou não de SRD e valor esperado da estimativa de $H\left(H_{g e n}\right)$. 
As Figuras 6.18, 6.19 e 6.20 apresentam as estimativas pelo método de AbryVeitch, em função de $H_{\text {gen }}$, para séries com $N=256, N=4096$ e $N=65535$, respectivamente. Para melhor visualização dos resultados, foram plotadas no mesmo gráfico as estimativas de $H$ tanto para $j_{\text {min }}=1$ quanto para $j_{\min }=$ $j_{\min }^{E Q M}$. Pelo mesmo motivo, os gráficos das estimativas para as séries sem e com SRD são apresentados conjuntamente na mesma figura. Cada ponto nos gráficos corresponde a uma das 8 realizações obtidas para cada valor de $H_{g e n}$.

Embora as Figuras 6.18 e 6.19 sejam de interesse para estudo dos casos com estimativas com número reduzido de amostras, é a Figura 6.20 que possibilita melhor contemplar o comportamento assintótico do estimador para estimações com número de amostras suficientemente grande. A Figura 6.20a demonstra claramente que as estimativas com $j_{\min }=1$ apresentam menor dispersão do que as estimativas com $j_{\text {min }}=j_{\text {min }}^{E Q M}$, o que, por sua vez, é traduzido por um maior viés, especialmente para o valor de $H_{\text {gen }}<0,7$. Para os casos de estimativas realizadas sobre séries com SRD, conforme Figuras 6.20b-d, é ainda mais nítido a menor dispersão das estimativas utilizando-se $j_{\text {min }}=1$, mas também é ainda mais notória a correção do viés destas estimativas utilizando-se de $j_{\min }=j_{\min }^{E Q M}$. 


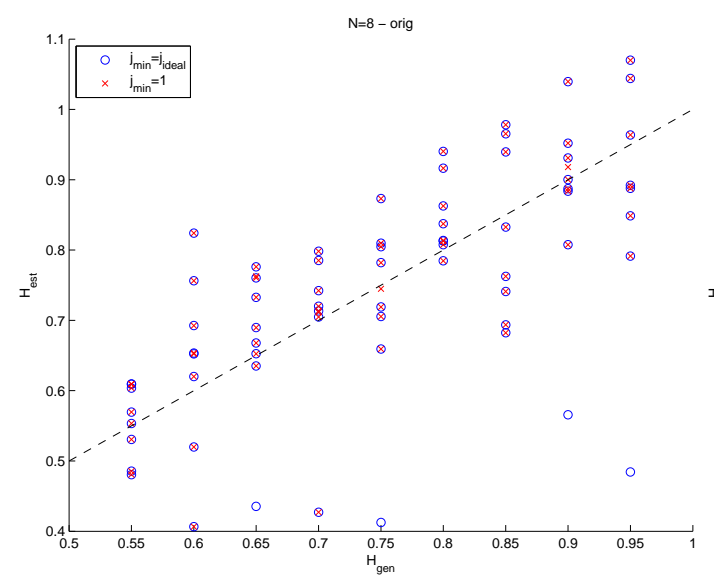

(a) Séries originais sem SRD

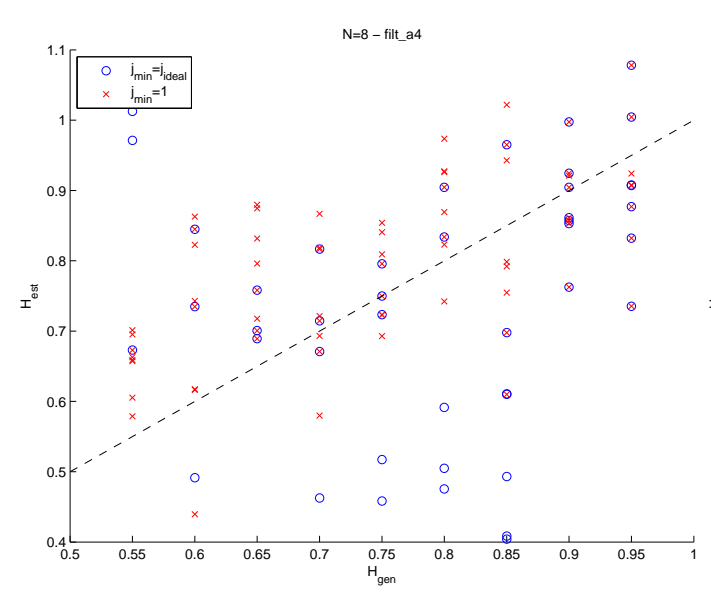

(c) Séries com SRD - $f_{0} \approx 0,4$

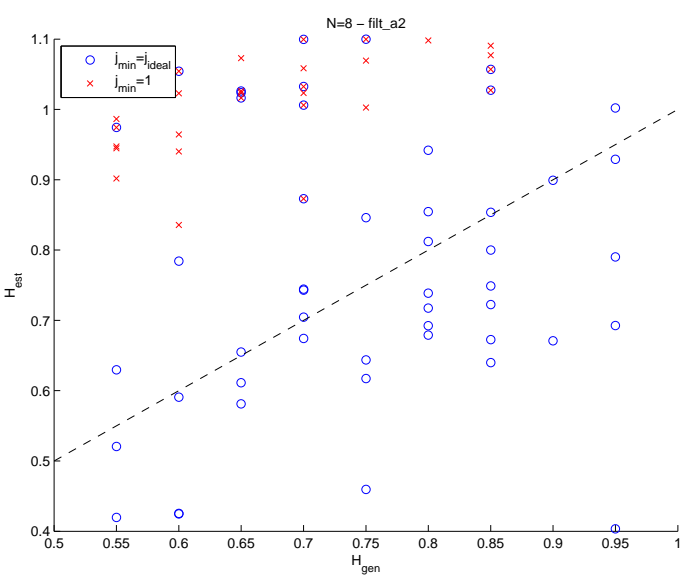

(b) Séries com SRD - $f_{0} \approx 0,2$

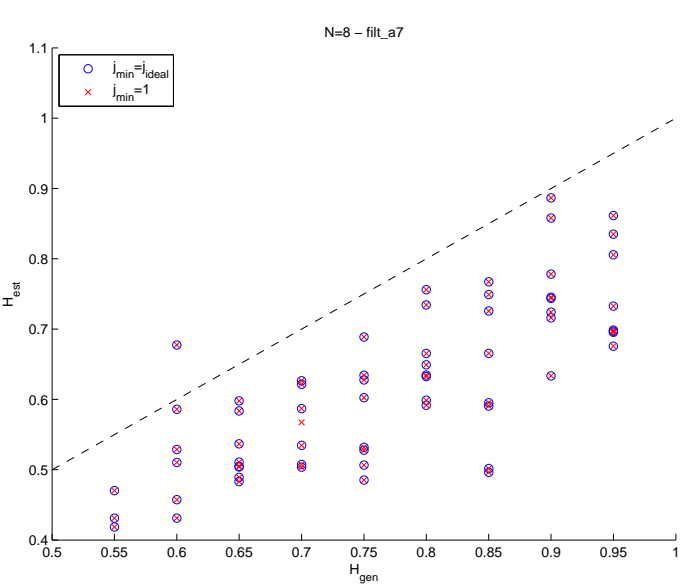

(d) Séries com SRD - $f_{0} \approx 0,7$

Figura 6.18: Estimativas pelo método de Abry-Veitch em função de $H_{\text {gen }}$ para $j_{\min } \in\left\{1, j_{\min }^{E Q M}\right\}$ e $N=256$. 


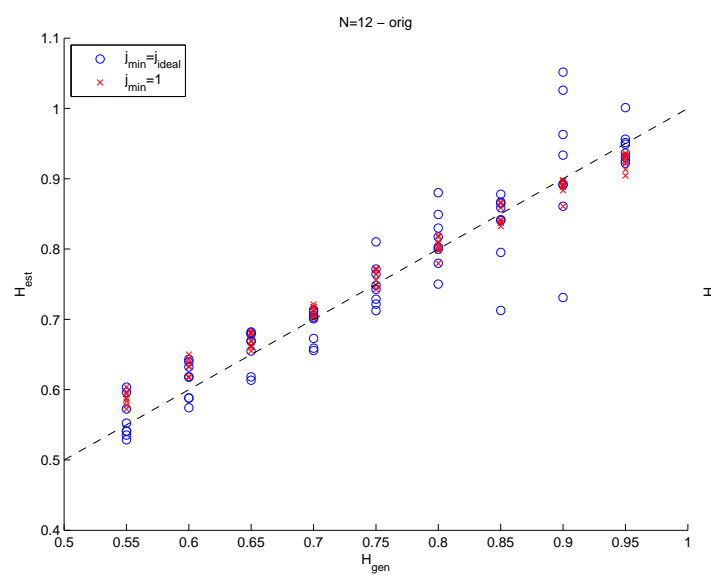

(a) Séries originais sem SRD

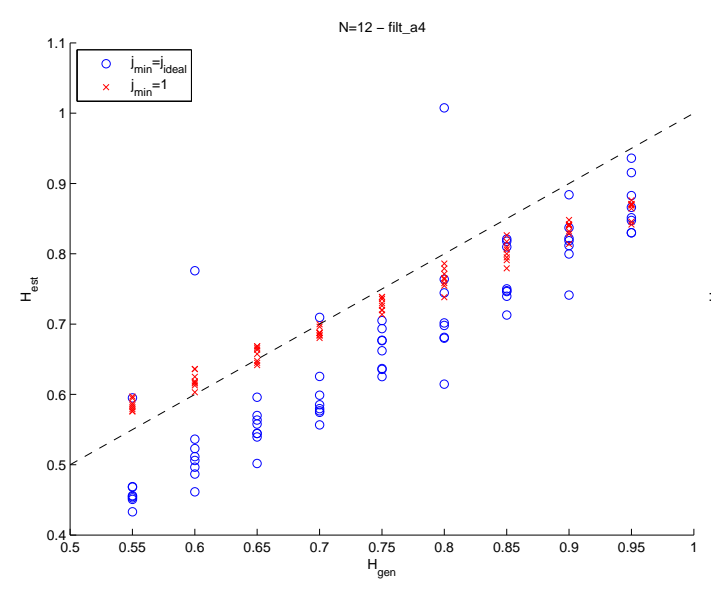

(c) Séries com SRD - $f_{0} \approx 0,4$

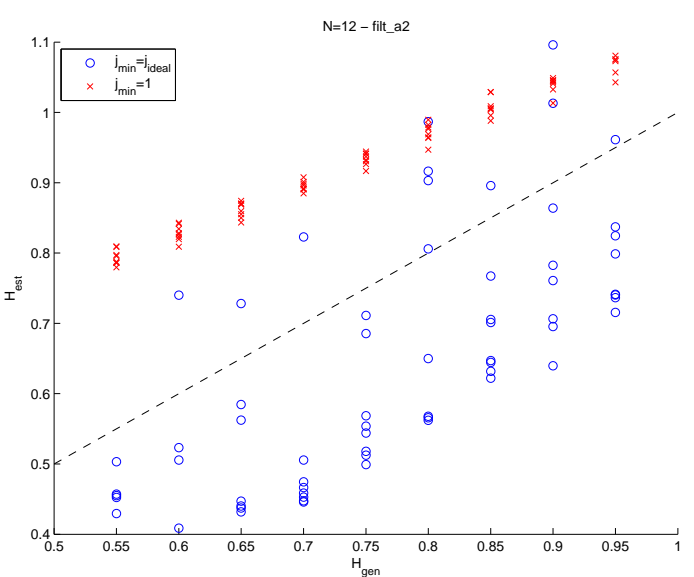

(b) Séries com SRD - $f_{0} \approx 0,2$

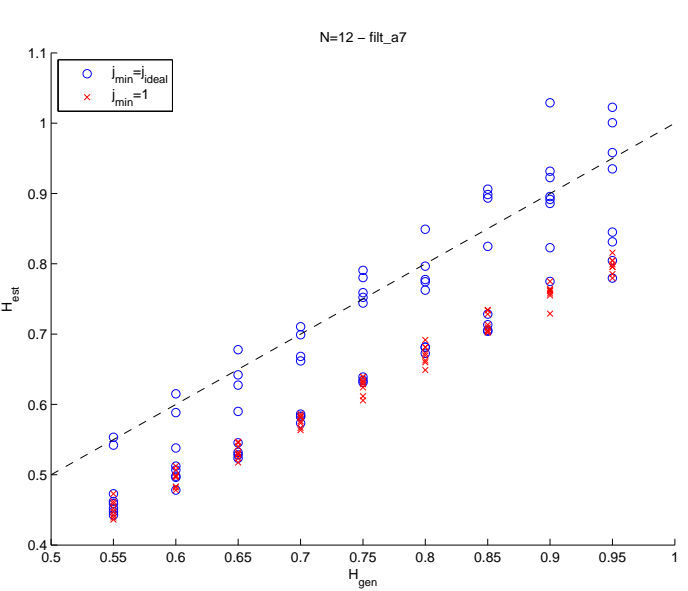

(d) Séries com SRD - $f_{0} \approx 0,7$

Figura 6.19: Estimativas pelo método de Abry-Veitch em função de $H_{\text {gen }}$ para $j_{\min } \in\left\{1, j_{\min }^{E Q M}\right\}$ e $N=4096$ 


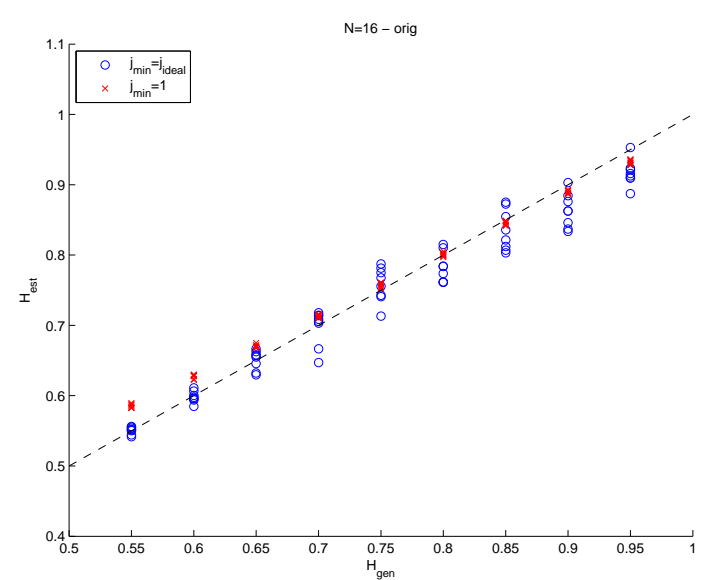

(a) Séries originais sem SRD

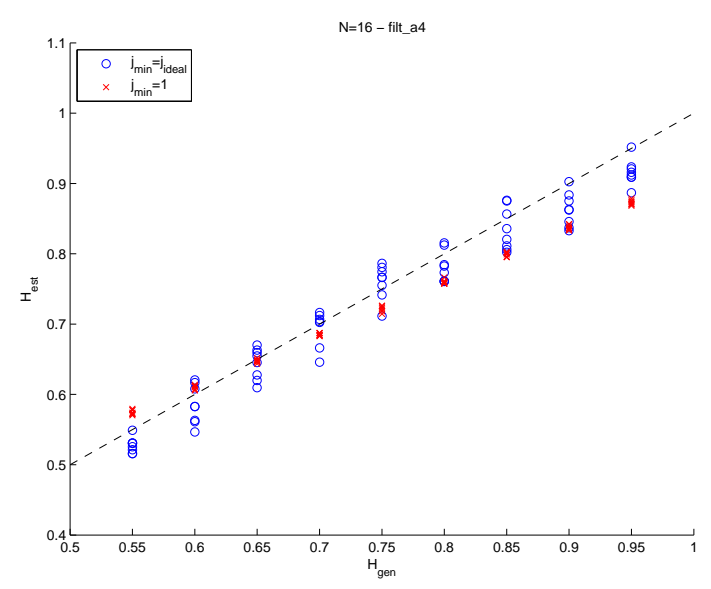

(c) Séries com SRD - $f_{0} \approx 0,4$

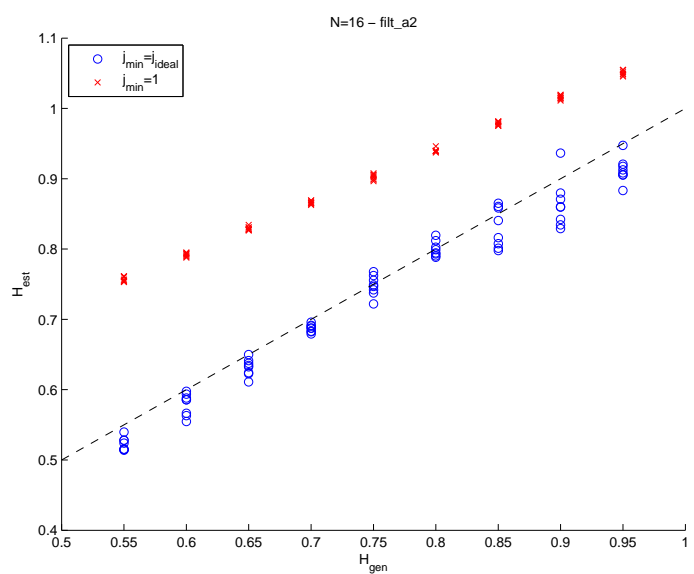

(b) Séries com SRD - $f_{0} \approx 0,2$

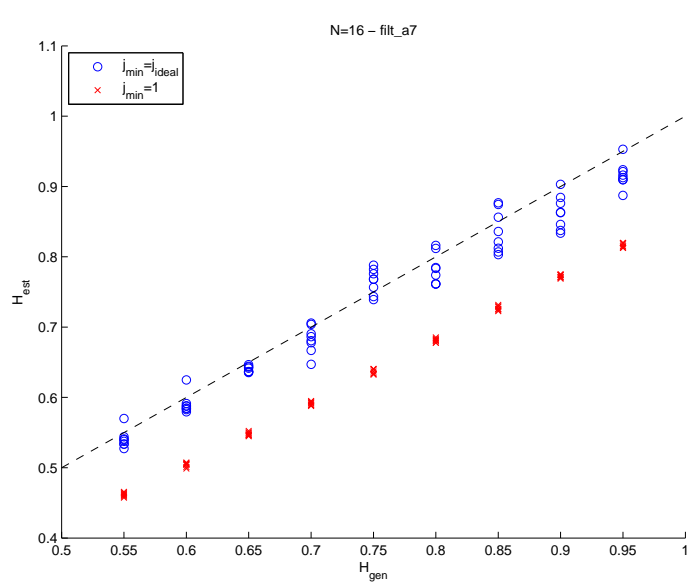

(d) Séries com SRD - $f_{0} \approx 0,7$

Figura 6.20: Estimativas pelo método de Abry-Veitch em função de $H_{\text {gen }}$ para $j_{\min } \in\left\{1, j_{\min }^{E Q M}\right\}$ e $N=65536$. 
Tendo sido explorado o comportamento do estimador em função do parâmetro $j_{\text {min }}$, e embora seja possível observar de certa maneira o comportamento do estimador em função de $N$ através dos gráficos das Figuras 6.18-6.20, uma melhor observação desse comportamento pode ser obtida redesenhando o gráfico com os mesmos resultados acima não em função de $H_{g e n}$, mas diretamente em função de $N$. Esse resultado ${ }^{3}$ pode ser observado nas Figuras 6.21 e 6.22 .

A Figura 6.21 explora, inicialmente, o comportamento das estimativas em função de $N$ crescente para o caso de $j_{\min }=1$. As linhas contínuas coloridas correspondem às estimativas de $H$, enquanto as linhas tracejadas coloridas correspondem às estimativas de $H$ mais ou menos o desvio padrão do conjunto de estimativas (representando a dispersão das mesmas). Pode-se observar para todos os valores de $H_{\text {gen }}$ e para todos os casos de séries com ou sem SRD que a convergência das estimativas é rápida, assim como a diminuição das dispersão. Entretanto, é evidente o viés do estimador, principalmente nos casos em que há componente SRD (Figuras 6.21b-d).

Já o comportamento das estimativas para o caso em que $j_{\min }=j_{\min }^{E Q M}$, conforme Figura 6.22, é tal que, embora a dispersão decaia em menor velocidade do que no caso anterior, bem como a convergência para o valor esperado seja mais lenta, as estimativas efetivamente convergem para o valor esperado, não apresentando viés na mesma magnitude que aquele apresentado para o caso em que $j_{\min }=1$, especialmente nos casos em que há componente SRD (Figuras 6.22bd). É digno de nota, contudo, que o comportamento do viés para o caso em que $H=0,9$ é consideravelmente pior do que para os outros valores de $H$, sendo necessária investigação futura para compreender tal comportamento.

\footnotetext{
${ }^{3}$ Esta análise é análoga à realizada no artigo [34].
} 


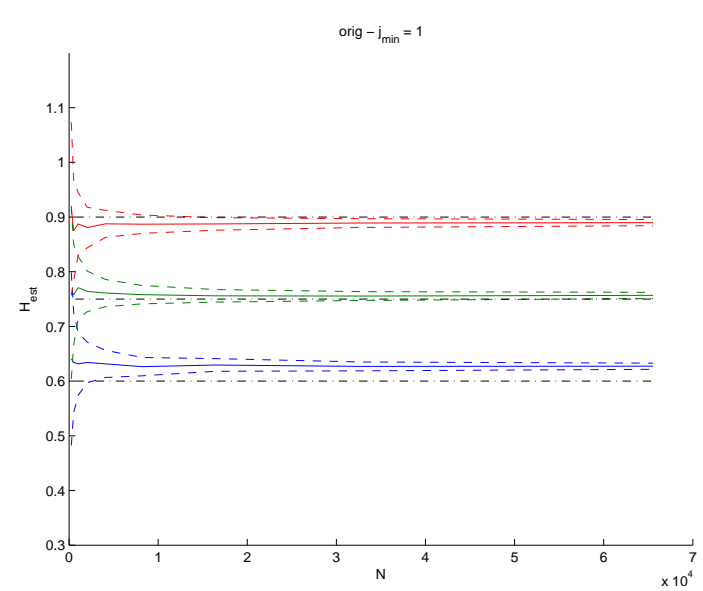

(a) Séries originais sem SRD

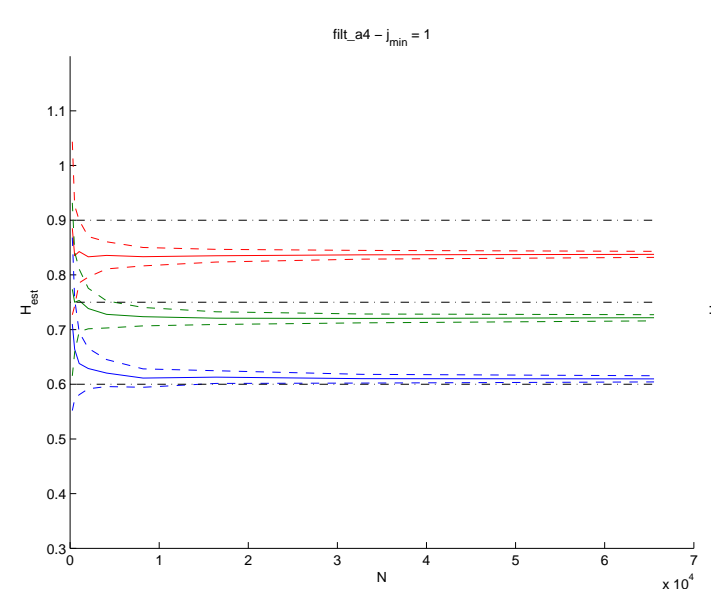

(c) Séries com SRD - $f_{0} \approx 0,4$

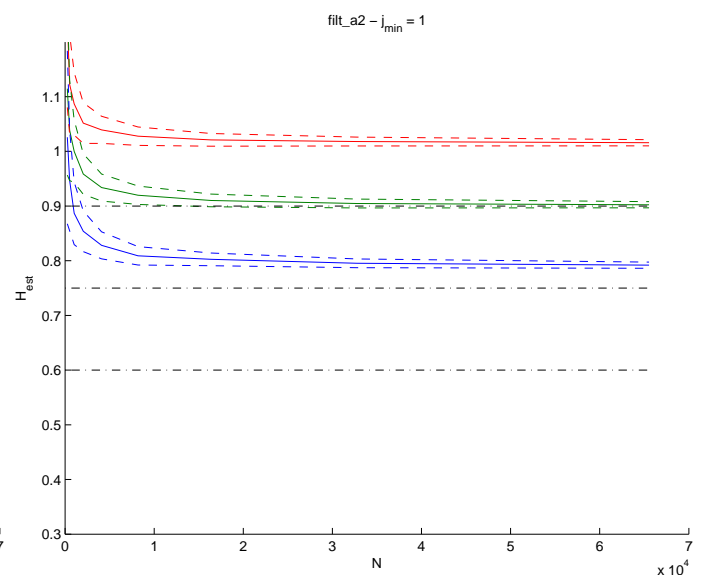

(b) Séries com SRD - $f_{0} \approx 0,2$

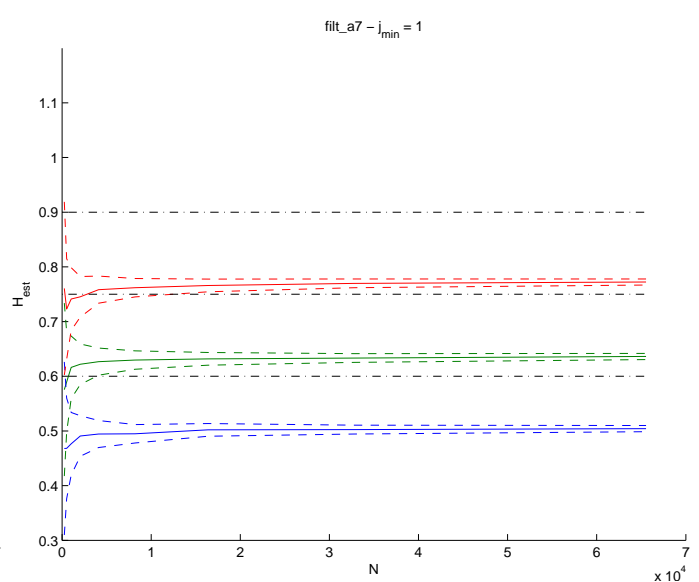

(d) Séries com SRD - $f_{0} \approx 0,7$

Figura 6.21: Estimativas pelo método de Abry-Veitch em função de $N$ para $H_{\text {gen }} \in\{0,6 ; 0,75 ; 0,9\}$ e $j_{\text {min }}=1$. 


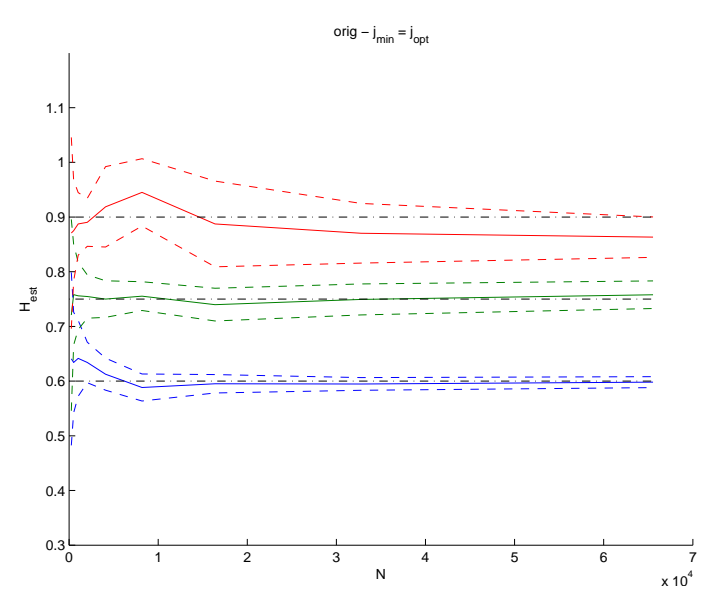

(a) Séries originais sem SRD

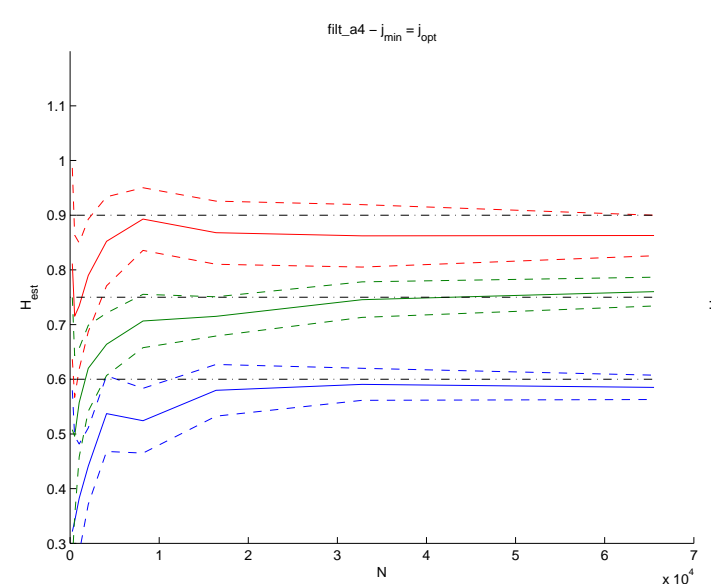

(c) Séries com SRD - $f_{0} \approx 0,4$

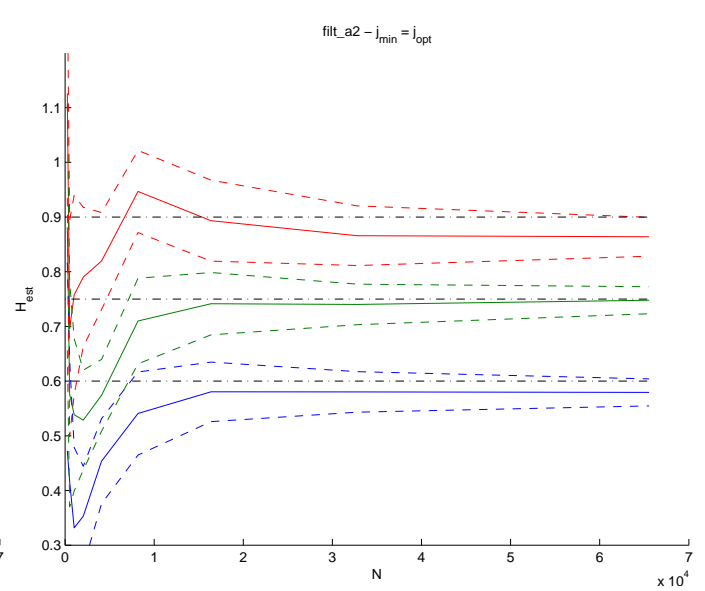

(b) Séries com SRD - $f_{0} \approx 0,2$

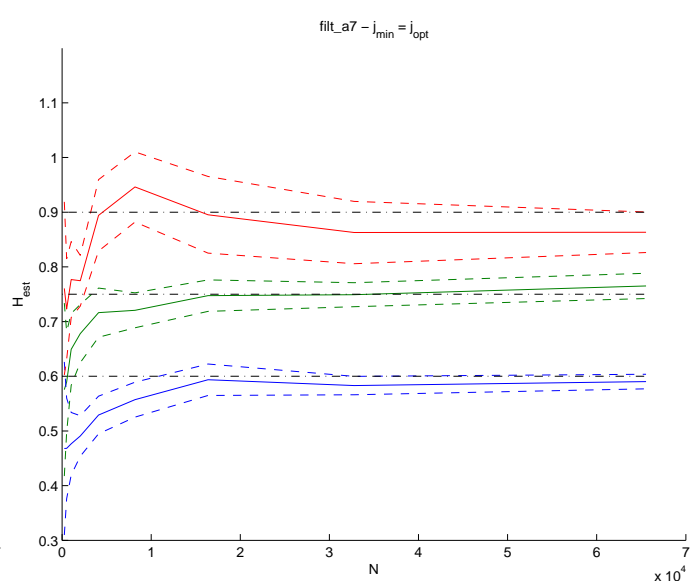

(d) Séries com SRD - $f_{0} \approx 0,7$

Figura 6.22: Estimativas pelo método de Abry-Veitch em função de $N$ para

$$
H_{\text {gen }} \in\{0,6 ; 0,75 ; 0,9\} \text { e } j_{\text {min }}=j_{\text {min }}^{E Q M} \text {. }
$$




\subsubsection{Exploração dos resultados}

A fim de aprofundar os resultados obtidos na Seção 6.2.2, buscou-se compreender os comportamentos observados das estimativas analisando-se os espectros wavelet de algumas das realizações em estudo. Desta maneira, para cada valor de $H_{\text {gen }} \mathrm{em}$ estudo na Seção anterior, foram representados em uma mesma figura os espectros wavelet de quatro realizações (do total de oito realizações geradas para cada valor de $\left.H_{g e n}\right)$. Cada gráfico em uma Figura corresponde a uma realização, sendo que os diversos espectros wavelet plotados em um mesmo gráfico correspondem à realização original (sem SRD) e às realizações com adição da componente SRD. Em tais gráficos, deve-se realizar a seguinte leitura da legenda:

- 0 (azul): série original sem SRD

- 2 (verde): série com $\mathrm{SRD}-f_{0} \approx 0,2$

- 4 (vermelho): série com $\mathrm{SRD}-f_{0} \approx 0,4$

- 7 (ciano): série com SRD - $f_{0} \approx 0,7$

Adicionalmente, as linhas tracejadas correspondem a retas ajustadas aos espectros wavelet dos gráficos $\left(\operatorname{com} j_{\min }=j_{\min }^{E Q M}\right)$. Uma linha tracejada que não tenha sua origem na escala mais à esquerda de um gráfico corresponde a uma reta ajustada com $j_{\text {min }} \neq 1$

As Figuras 6.23-6.25 apresentam os espectros wavelet para as séries com 256 pontos $\left(H_{\text {gen }} \in\{0,6 ; 0,75 ; 0,9\}\right.$, respectivamente), as Figuras 6.26-6.28 apresentam as séries com 4096 pontos $\left(H_{\text {gen }} \in\{0,6 ; 0,75 ; 0,9\}\right.$, respectivamente), e as Figuras 6.29-6.31 apresentam as séries com 65536 pontos $\left(H_{\text {gen }} \in\{0,6 ; 0,75 ; 0,9\}\right.$, respectivamente).

Os espectros das Figuras 6.23-6.25, como pode ser observado, são de tal forma que as retas ajustadas são de declividade bastante variável, tanto entre diferentes realizações, como para o caso de uma mesma realização com ou sem componentes SRD. Isso justifica a grande dispersão das estimativas na Figura 6.18. Nas Figuras 6.26-6.28, já é possível verificar com mais clareza que parte do espectro é invariante mesmo com a adição de SRD. No outro extremo, os espectros das Figuras 6.29-6.31 exibem um comportamento bastante definido, onde claramente as estimativas tanto entre realizações distintas como para uma mesma realização com ou sem componente SRD são bastante similares, tendo em vista as retas ajustadas de mesma declividade. 


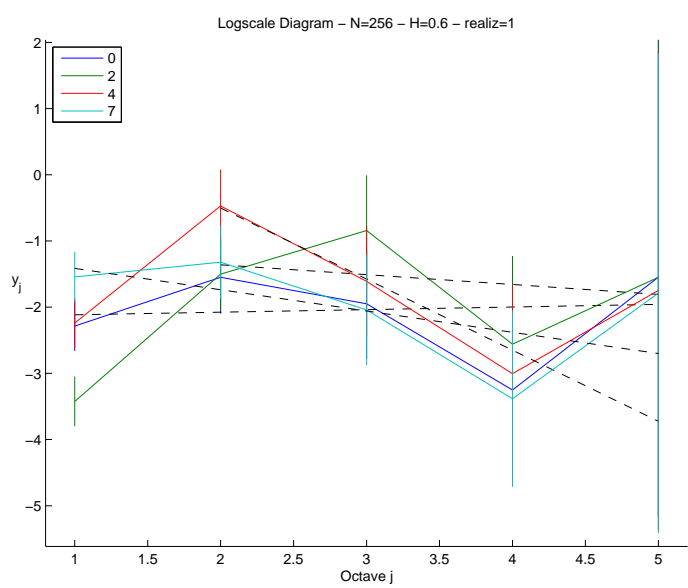

(a)

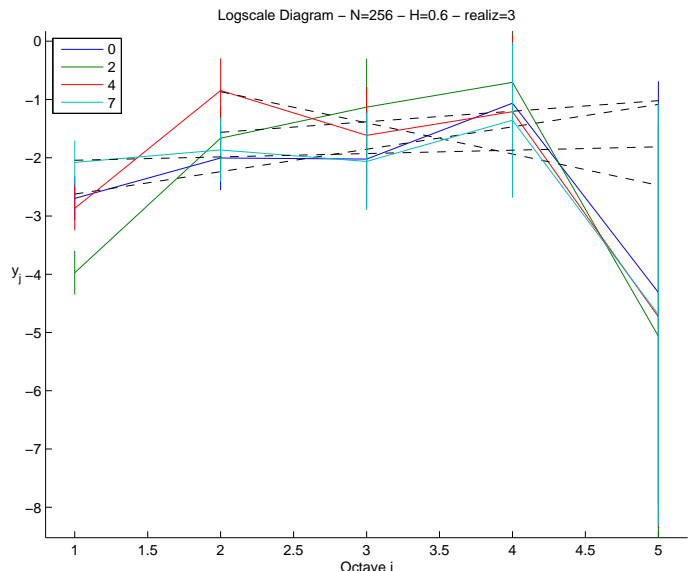

(c)

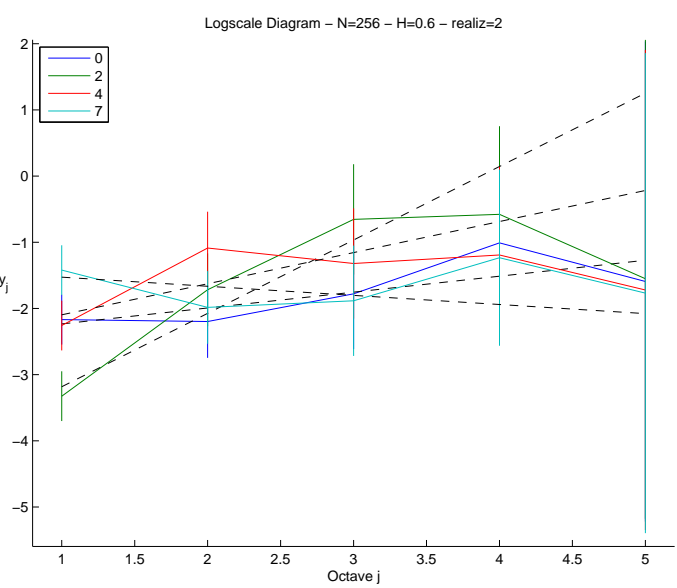

(b)

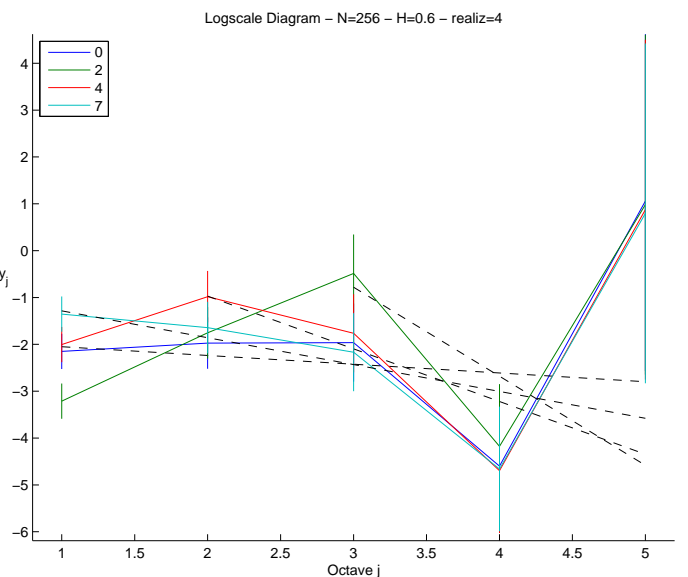

(d)

Figura 6.23: Espectro wavelet de quatro realizações distintas com $N=256$ e $H=0,6$. 


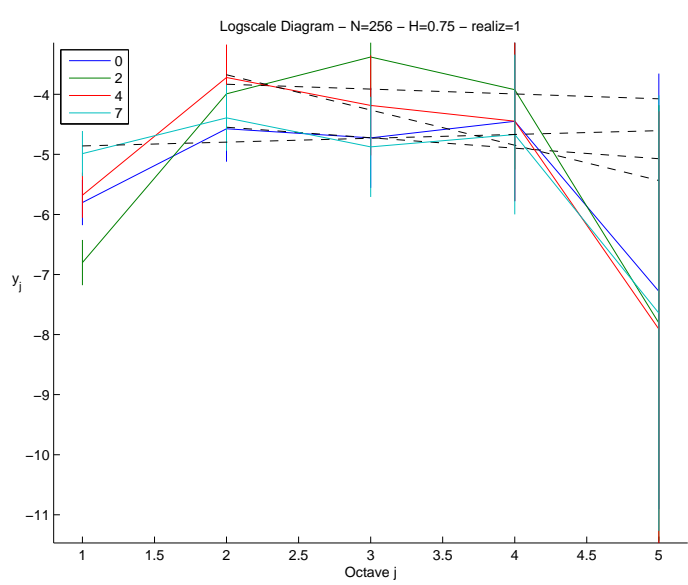

(a)

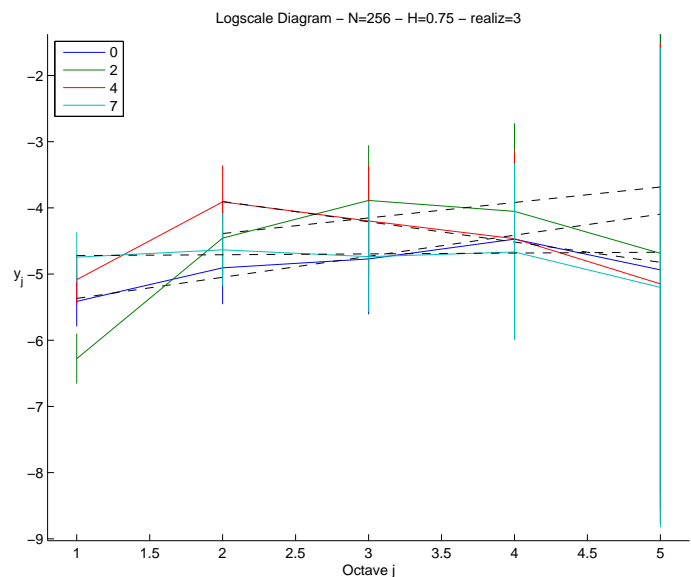

(c)

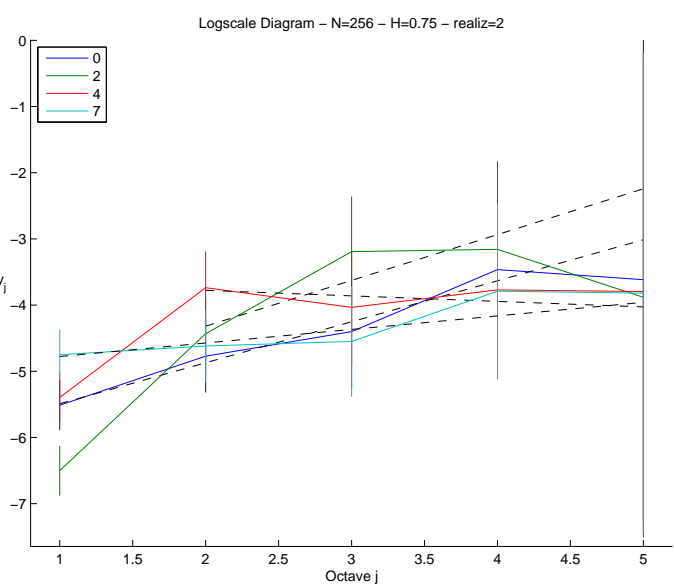

(b)

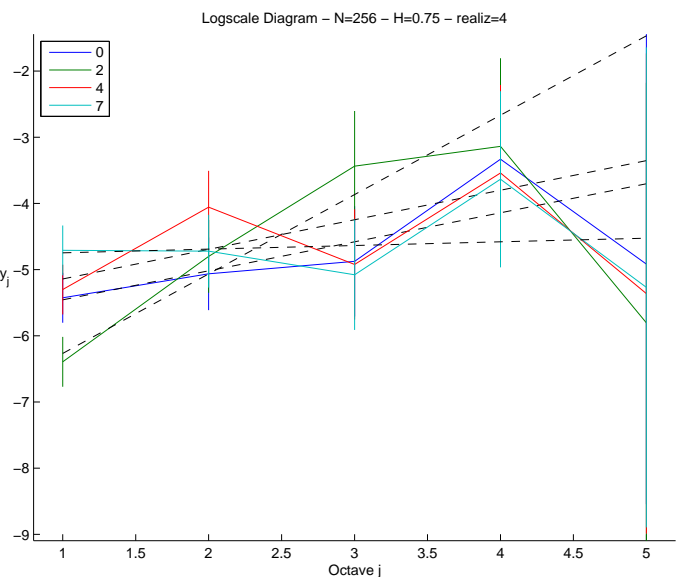

(d)

Figura 6.24: Espectro wavelet de quatro realizações distintas com $N=256$ e $H=0,75$. 


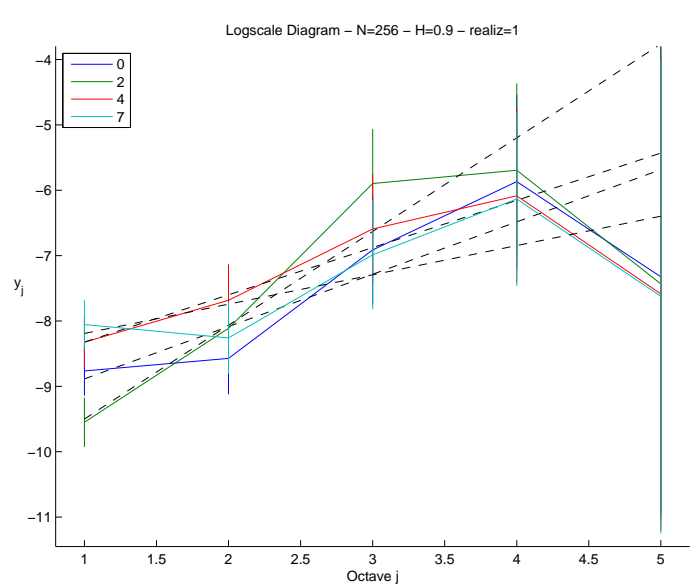

(a)

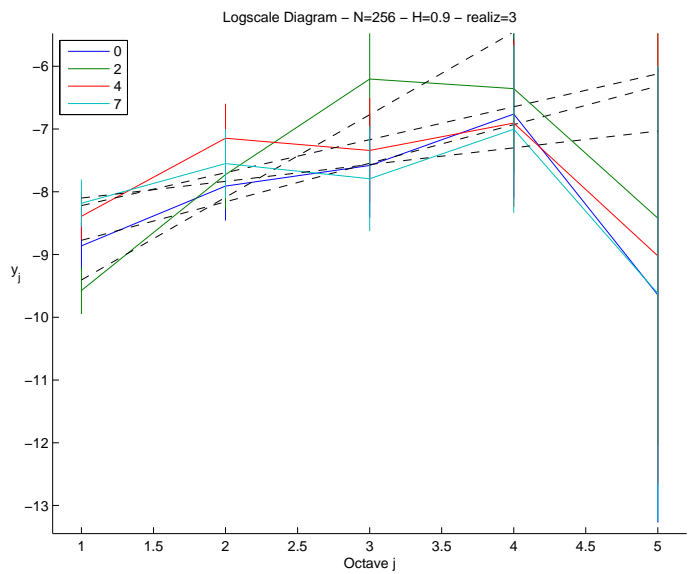

(c)

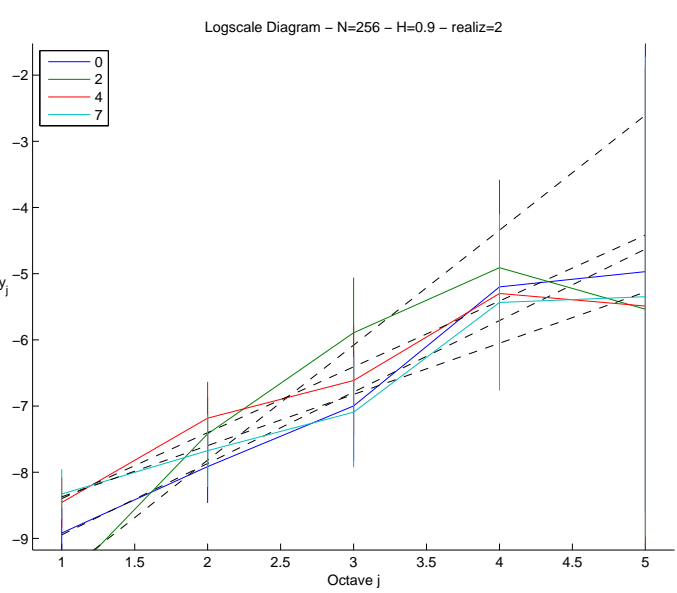

(b)

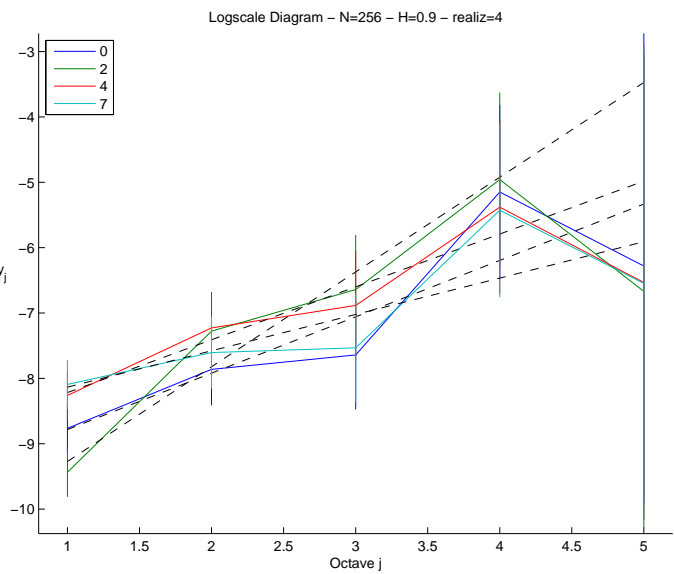

(d)

Figura 6.25: Espectro wavelet de quatro realizações distintas com $N=256$ e $H=0,9$. 


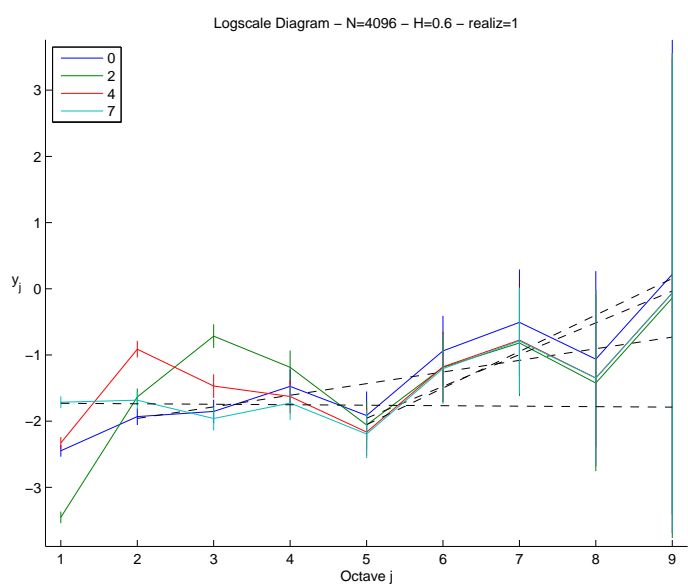

(a)

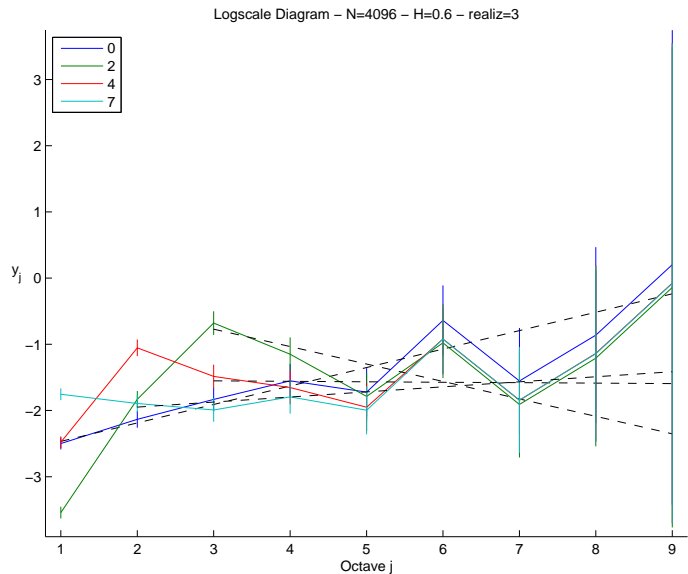

(c)

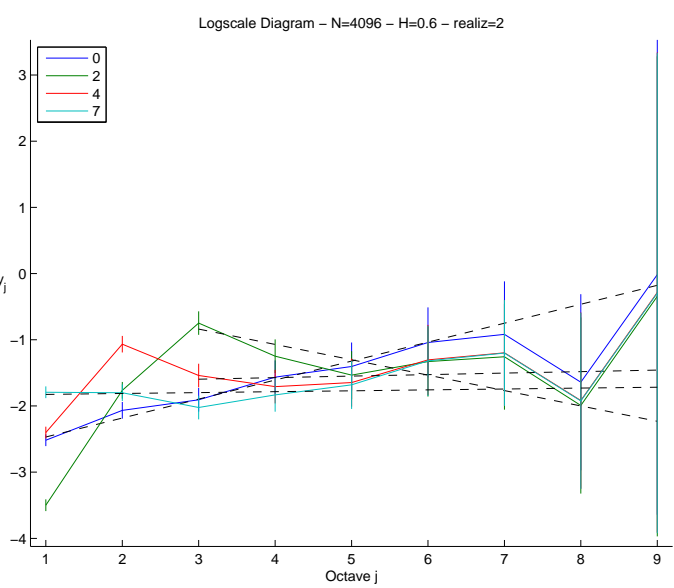

(b)

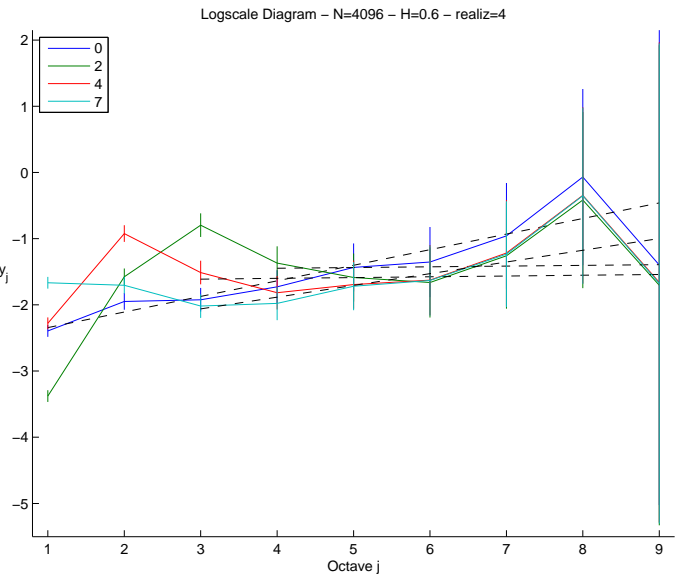

(d)

Figura 6.26: Espectro wavelet de quatro realizações distintas com $N=4096$ e $H=0,6$. 


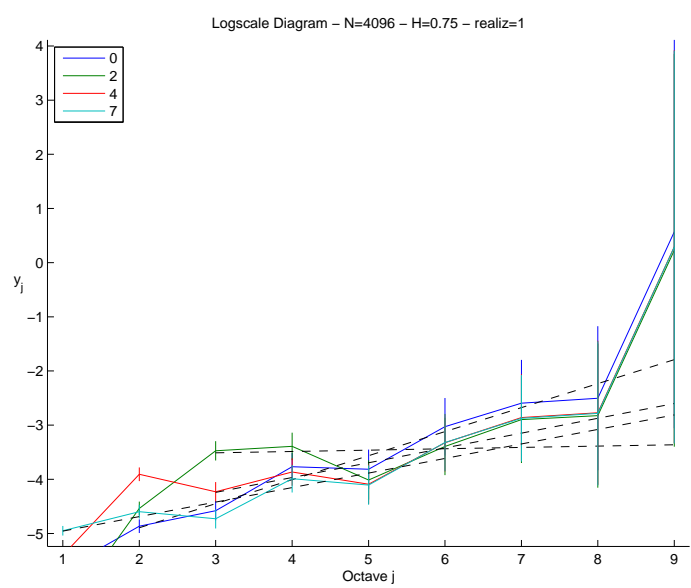

(a)

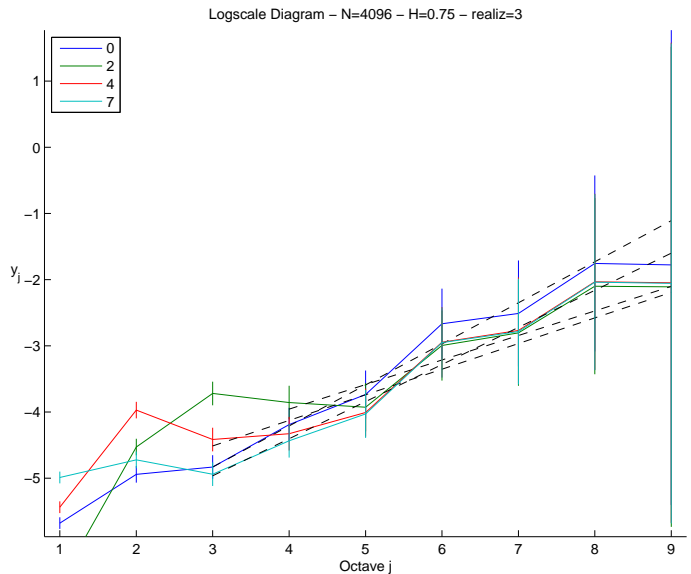

(c)

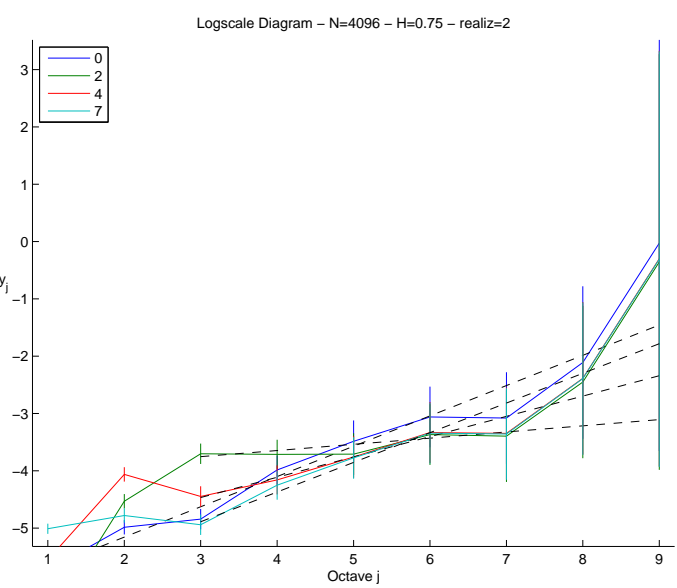

(b)

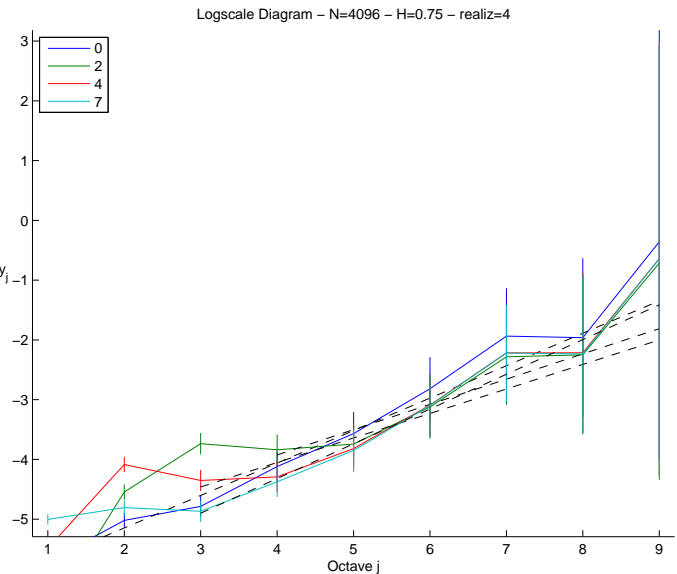

(d)

Figura 6.27: Espectro wavelet de quatro realizações distintas com $N=4096$ e $H=0,75$. 


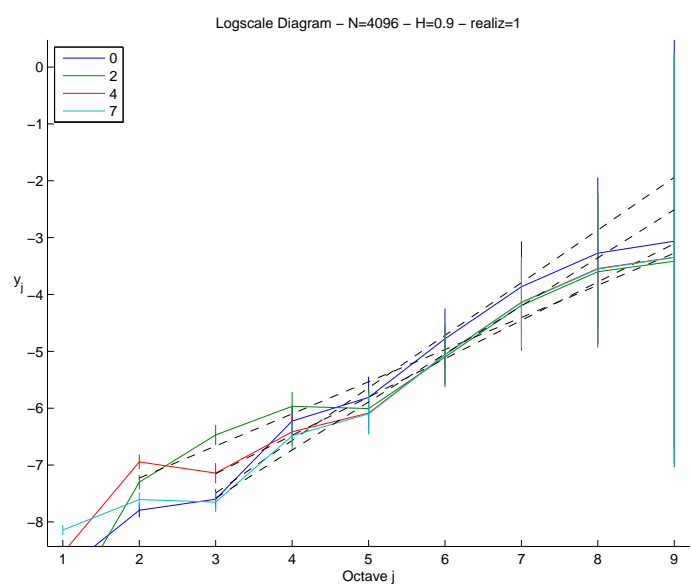

(a)

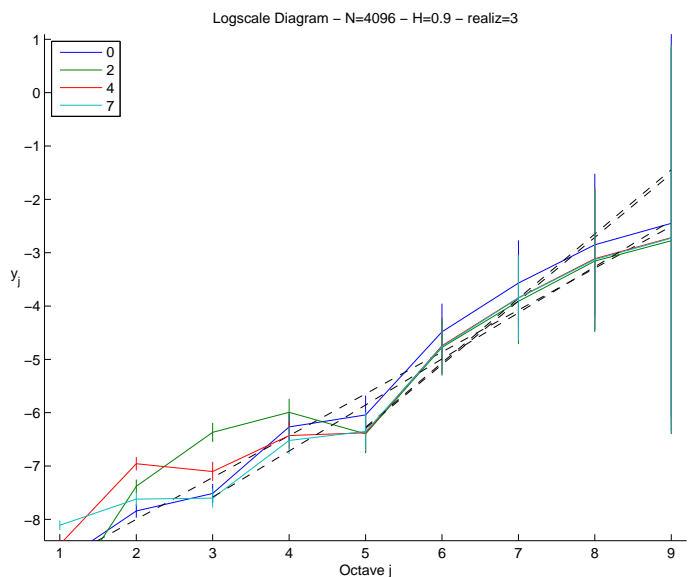

(c)

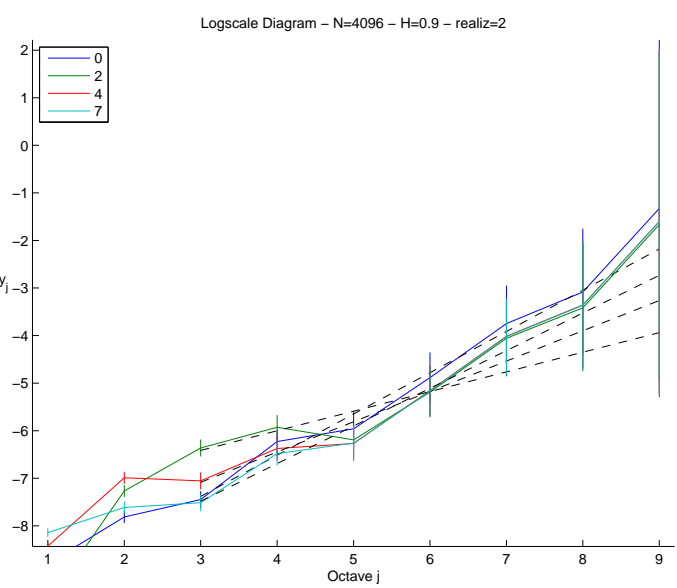

(b)

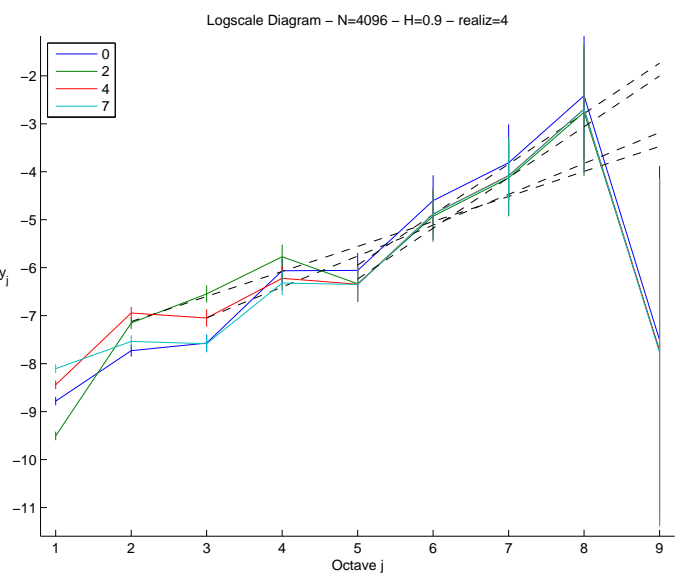

(d)

Figura 6.28: Espectro wavelet de quatro realizações distintas com $N=4096$ e $H=0,9$. 


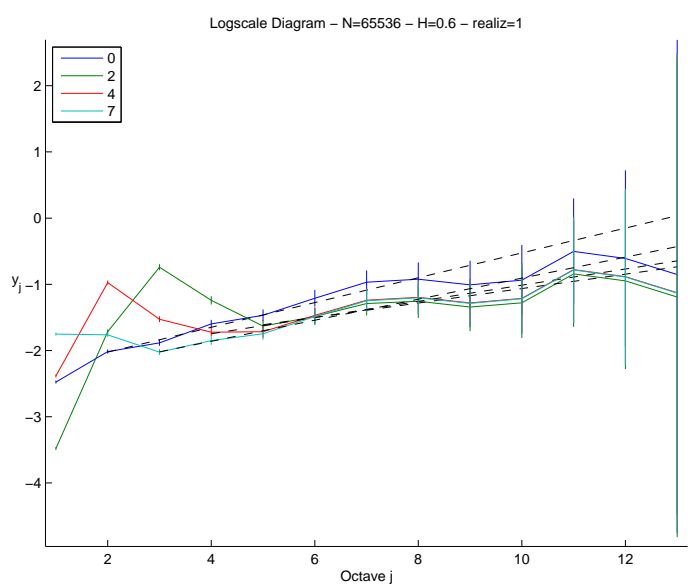

(a)

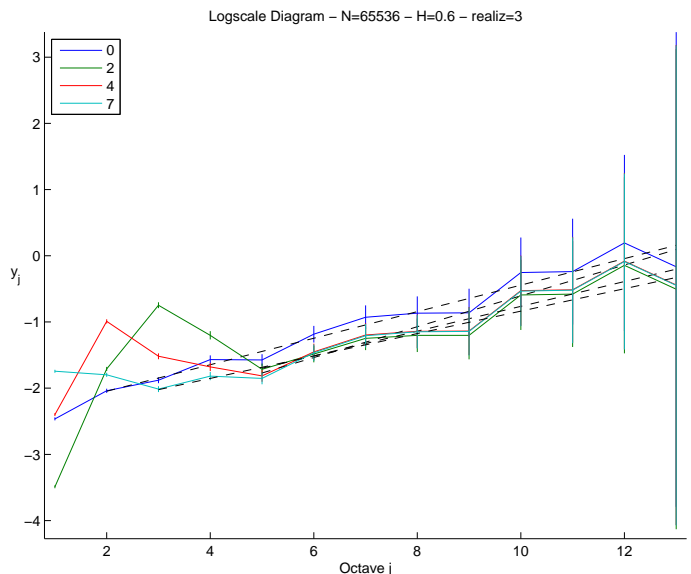

(c)

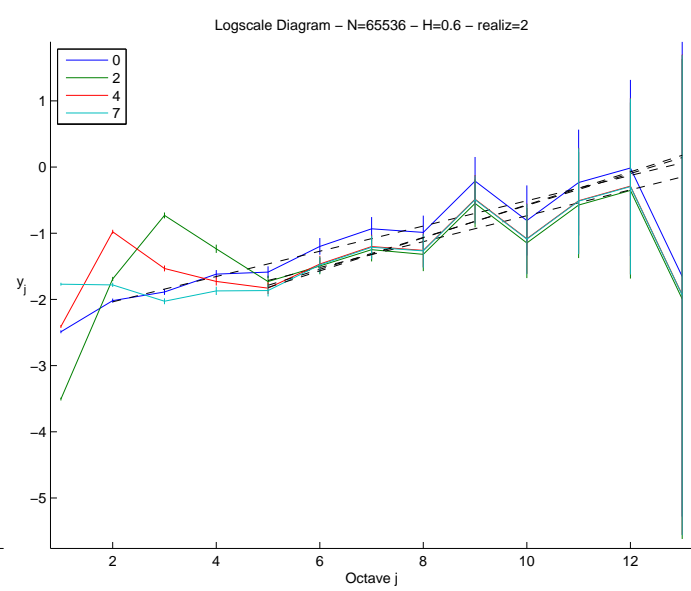

(b)

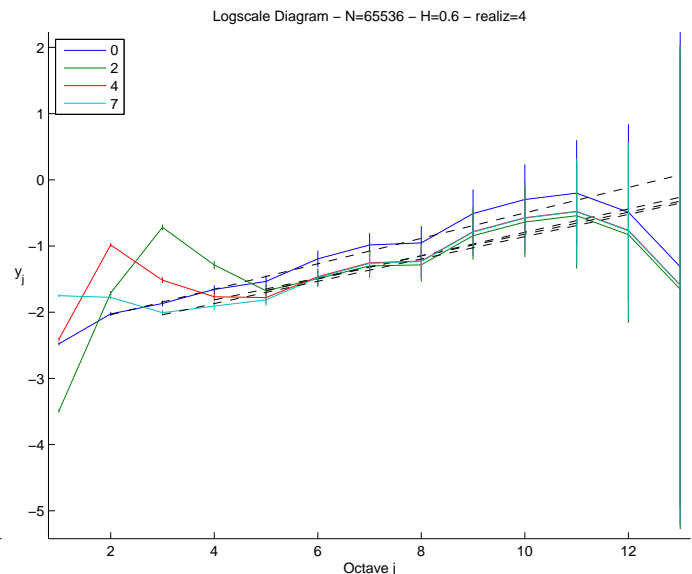

(d)

Figura 6.29: Espectro wavelet de quatro realizações distintas com $N=65536$ e $H=0,6$. 


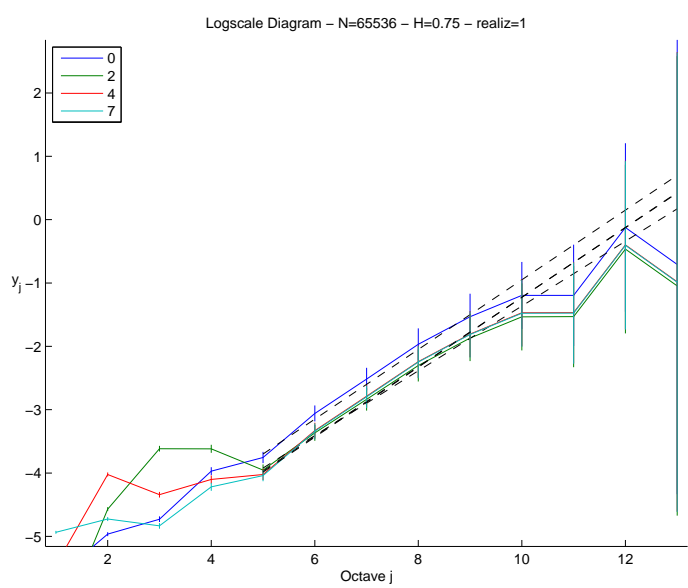

(a)

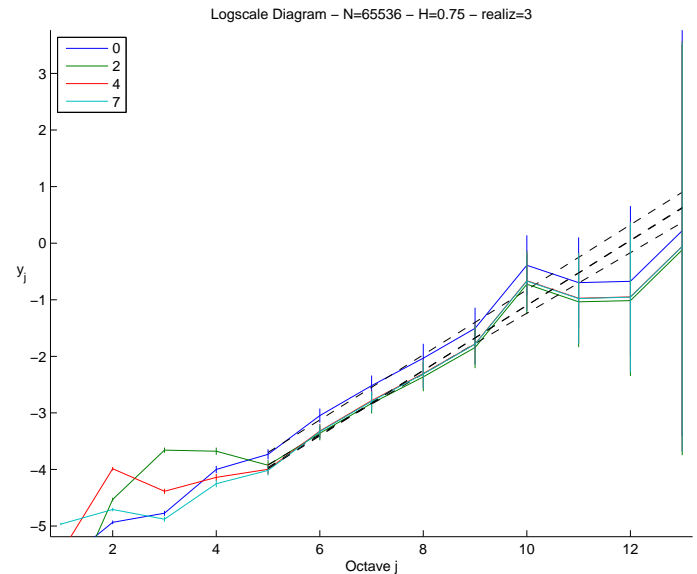

(c)

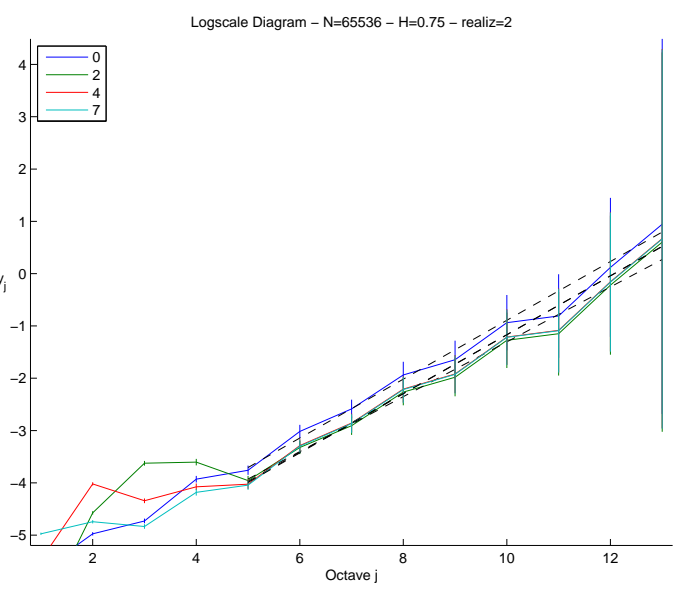

(b)

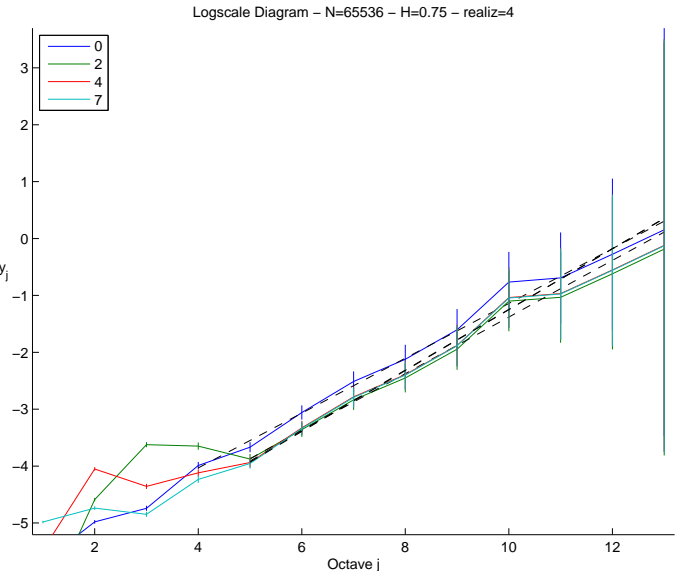

(d)

Figura 6.30: Espectro wavelet de quatro realizações distintas com $N=65536$ e $H=0,75$. 


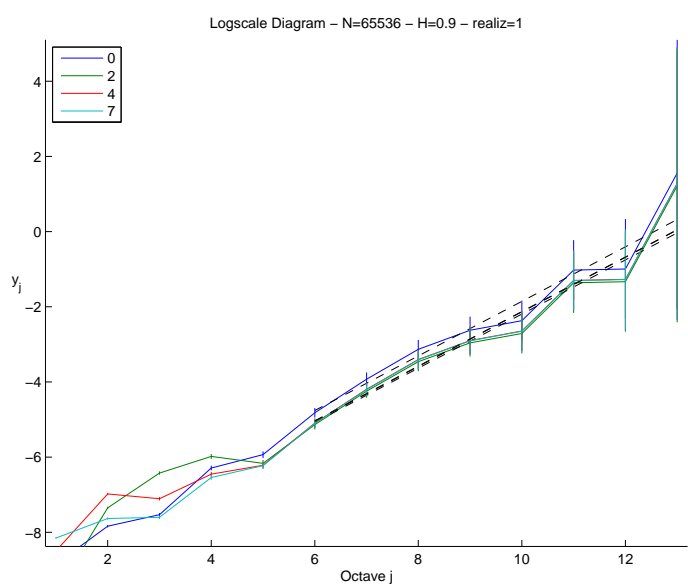

(a)

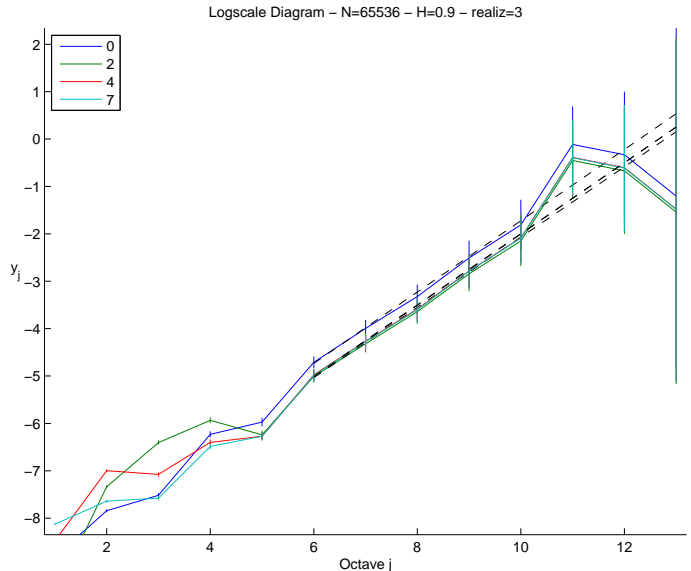

(c)

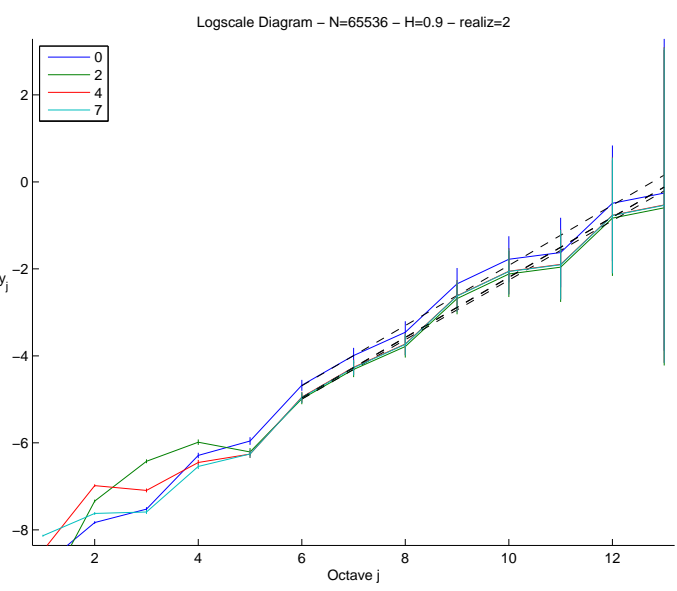

(b)

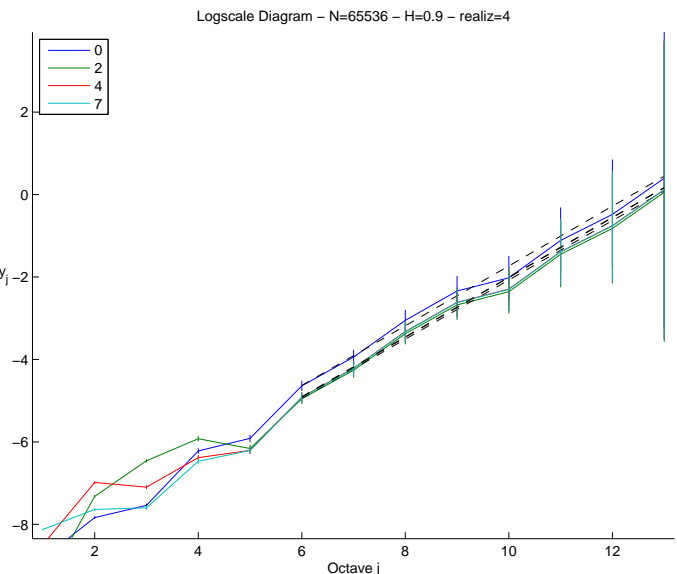

(d)

Figura 6.31: Espectro wavelet de quatro realizações distintas com $N=65536$ e $H=0,9$. 
Além disso, observa-se em tais gráficos que, em geral, $j_{\min } \neq 1$ e que as retas ajustadas têm início apenas na região em que os espectros para as realizações com diferentes componentes SRD são razoavelmente paralelos entre si. Isso sugere que pode haver, a partir de um determinado valor de $N$, uma determinada escala característica tal que, impondo-se $j_{\min }$ como sendo igual a essa escala, obter-se-á as estimativas de $H$ com menor viés possível. Por outro lado, como são utilizados menos pontos do espectro para a estimativa, e os pontos utilizados pertencem a uma região do espectro em que as estimativas do espectro são mais incertas, isso traduz-se no aumento da dispersão das estimativas de $H$ para o caso em que $j_{\text {min }}=j_{\min }^{E Q M}$, conforme explorado na Figura 6.22. É interessante notar, entretanto, que $j_{\min }^{E Q M}$ cresce com o valor de $H$, de forma que para $H_{g e n}=0,9$, $j_{\min }^{E Q M}$ é tal que as retas ajustadas iniciam-se em escalas um tanto quanto acima da escala a partir do qual os espectros passam a poder ser considerados paralelos, resultando no viés observado na estimação.

\subsubsection{Síntese dos resultados}

A Tabela 6.2 apresenta uma síntese dos principais resultados expostos na Seção 6.2 .

Tabela 6.2: Resultados da Seção 6.2.

\begin{tabular}{|c|c|}
\hline Resultado & Descrição \\
\hline 1 & $\begin{array}{l}\text { Utilização de } j_{\min }=j_{\min }^{E Q M} \text { implica em correção do viés em troca de maior } \\
\text { dispersão das estimativas }\end{array}$ \\
\hline 2 & $\begin{array}{l}\text { Para estimativas com } N \text { reduzido, em geral } j_{\min }^{E Q M} \approx 1 \text {, surtindo pouco efeito } \\
\text { correção do viés a utilização de tal } j_{\min }^{E Q M}\end{array}$ \\
\hline 3 & $\begin{array}{l}\text { Gráficos da estimativa de } H \text { em função de } N \text { confirmam que o comportamento } \\
\left.\text { assintótico das estimativas (exceto para } H_{g e n}=0,9\right) \text { utilizando-se } j_{\min }=j_{\min }^{E Q M} \\
\text { tende a anular o viés observado para o caso em que } j_{\min }=1\end{array}$ \\
\hline 4 & $\begin{array}{l}\text { Gráficos da estimativa de } H \text { em função de } N \text { podem ser utilizados para deter- } \\
\text { minar o valor de } N \text { necessário para que a dispersão do estimador, para o caso } \\
j_{\min }=j_{\min }^{E Q M} \text {, tenha seu valor limitado. }\end{array}$ \\
\hline 5 & $\begin{array}{l}\text { Para } N \text { suficientemente grande, é possível identificar a região do espectro wa- } \\
\text { velet em que não há influência de SRD }\end{array}$ \\
\hline 6 & $\begin{array}{l}\text { Para } N \text { suficientemente grande, } j_{m i n}^{E Q M} \text { em geral coincide com o início da região } \\
\text { do espectro em que não há influência de SRD }\end{array}$ \\
\hline
\end{tabular}




\subsection{Análise da influência da variação do nível de SRD}

Uma das questões fundamentais da estimação em tempo de real de séries de teletráfego é a do número de amostras da série que serão utilizadas para a estimação. Idealmente, considerando-se uma série estacionária em sentido amplo, é desejável que sejam utilizadas todas as amostras disponíveis de uma série. Em caso de estimação em tempo real, esse número de amostras é incrementado com o tempo. As Figuras 6.21 e 6.22 demonstram tal fato: quanto mais amostras, menor a dispersão das estimativas e mais próximo tais estimativas estarão do comportamento assintótico.

Na prática, entretanto, as séries podem não apresentar comportamento estacionário, e a estimação utilizando um número grande de amostras pode ocasionar problemas de estimação causados pelo fato de estar utilizando-se amostras correspondentes de regimes distintos. Uma forma adotada para contornar tal problema é a utilização de janelamento, que consiste em realizar as estimações com uma janela de tamanho fixo de amostras, que é deslocada à medida que mais amostras são incorporadas à série. Desta maneira as estimações são realizadas sempre com as amostras mais recentes, ao mesmo tempo que amostras mais antigas são desprezadas. O artigo [35] apresenta em detalhes tal técnica, assim como apresenta outras que merecem uma investigação mais aprofundada (como, por exemplo, a utilização de filtro de Kalman).

O objetivo da Seção 6.3 é estender os resultados obtidos em [35, 36], verificando o comportamento do estimador de Abry-Veitch para o caso em que há mudança de regime da componente SRD da série.

\subsubsection{Condições experimentais}

Para verificar o comportamento transitório da estimação nos casos em que há mudança de regime, foi utilizado um esquema em que as séries de 65536 pontos descritas na Seção 6.2.1 foram concatenadas, duas a duas, de forma a simular alterações de regime. Essas novas séries foram agregadas segundo três grupos:

1. séries com valores idênticos de $H$ e de características de SRD;

2. séries com valores distintos de $H$, mas características idênticas de SRD

3. séries com valores idênticos de $H$, mas características distintas de SRD 
Embora o foco seja no estudo das séries acima descritas no grupo 3, os grupos 1 e 2 foram também analisados para fins de controle e comparação dos resultados com o grupo 3.

Os gráficos a serem apresentados na Seção 6.3.2 representam as estimativas de $H$ para as séries concatenadas duas a duas conforme os grupos acima apresentados. Tais séries, com 131072 amostras, tiveram o valor de $H$ estimado através de janelas deslizantes de tamanho 1024 e 65536, deslocadas em passos de 1024 amostras. O primeiro ponto de tais gráficos representa a estimativa de $H$ logo antes mudança de regime, sendo que as demais estimativas já representam as estimativas no novo regime. Tais gráficos foram gerados para as estimativas utilizando-se tanto $j_{\text {min }}=1$ como $j_{\text {min }}=j_{\text {min }}^{E Q M}$.

\subsubsection{Resultados obtidos}

As estimações referentes ao grupo com valores idênticos de $H$ e de características de SRD, realizadas para controle, são apresentadas nas Figuras 6.32-6.34. A linha negra tracejada indica o valor esperado da estimação $\left(H_{g e n}\right)$, a linha tracejada vermelha indica o valor médio das estimativas realizadas conforme indicado na Seção 6.3.1, e as linhas contínuas em verde e azul representam a dispersão das estimativas, sendo que a linha verde representa o valor médio das estimativas mais ou menos o desvio padrão das mesmas, e a linha azul representa a média dos valores máximo e mínimo conforme definidos no algoritmo de Abry-Veitch (vide Seção 4.3). Observa-se em tais gráficos que tanto o viés como a dispersão em todos os casos estão em conformidade com os resultados apresentado nas Seções anteriores. 


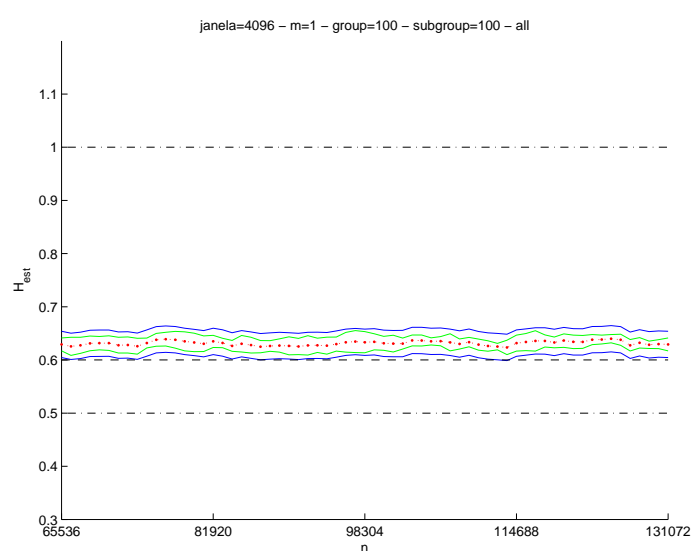

(a) $j_{\min }=1 / N=4096$

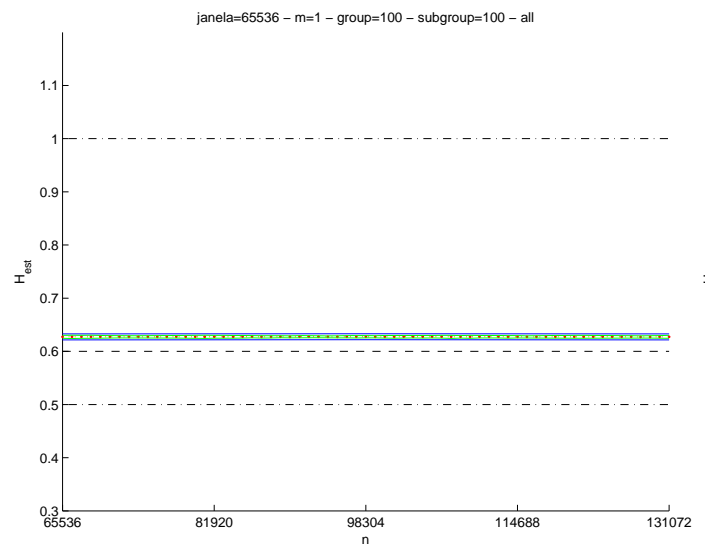

(c) $j_{\min }=1 / N=65536$

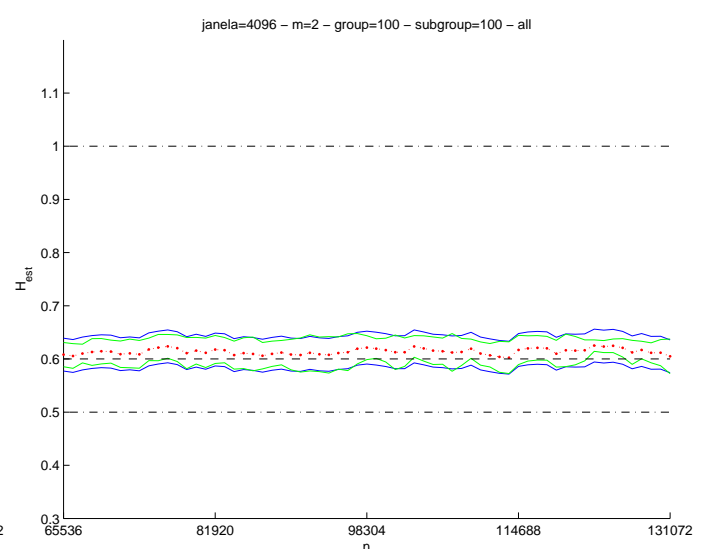

(b) $j_{\min }=j_{\min }^{E Q M} / N=4096$

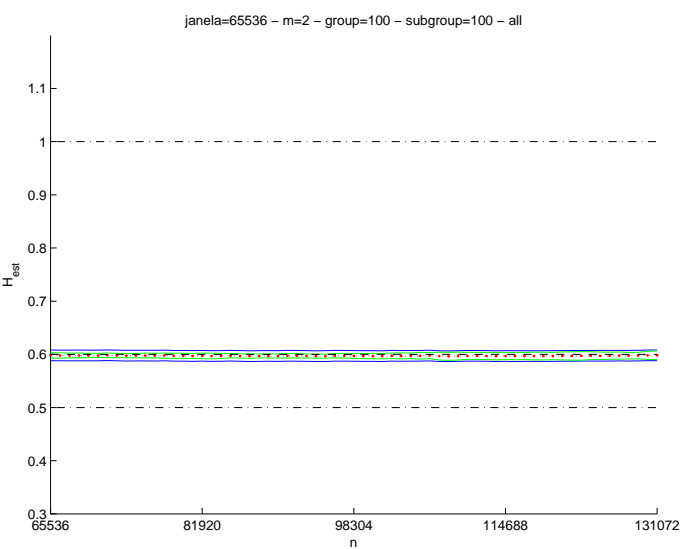

(d) $j_{\min }=j_{\min }^{E Q M} / N=65536$

Figura 6.32: Estimação de séries concatenadas (controle) $H=0,6 ; f_{0}=0 \Longrightarrow H=0,6 ; f_{0}=0$. 


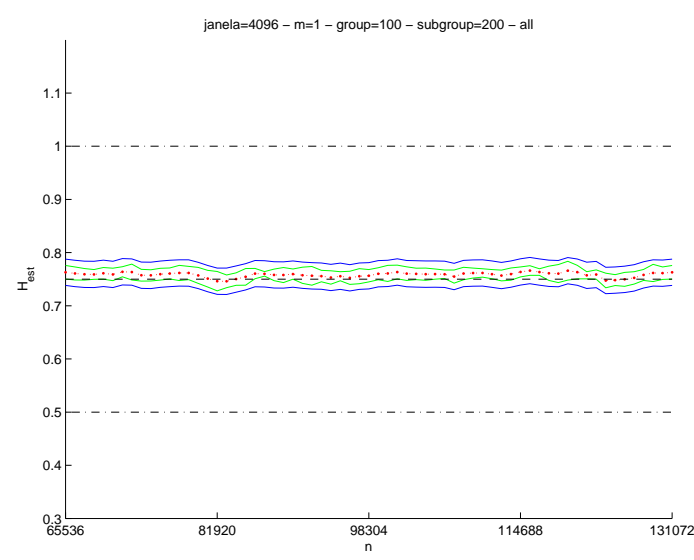

(a) $j_{\min }=1 / N=4096$

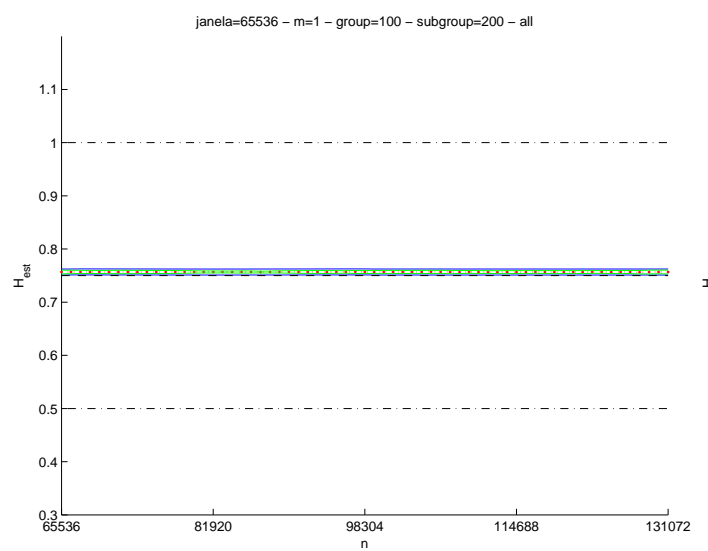

(c) $j_{\min }=1 / N=65536$

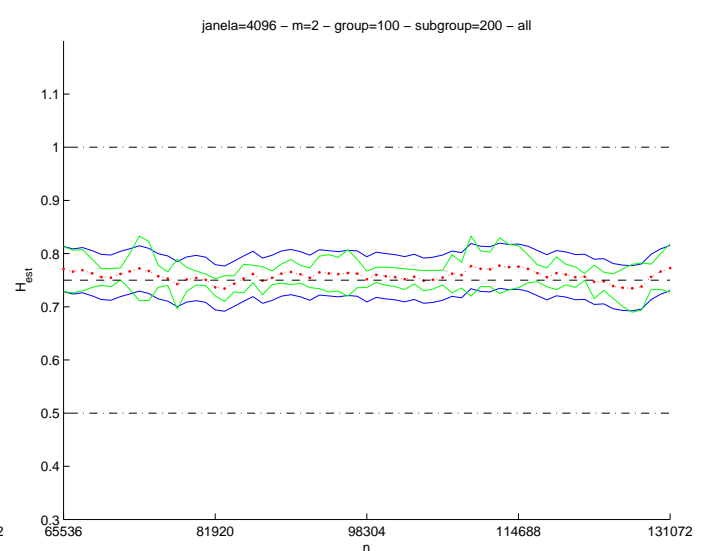

(b) $j_{\min }=j_{\min }^{E Q M} / N=4096$

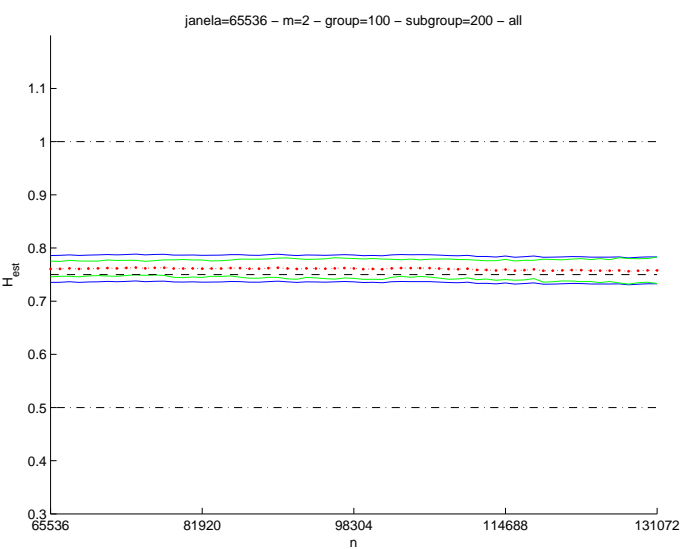

(d) $j_{\min }=j_{\min }^{E Q M} / N=65536$

Figura 6.33: Estimação de séries concatenadas (controle) -

$$
H=0,75 ; f_{0}=0 \Longrightarrow H=0,75 ; f_{0}=0 .
$$




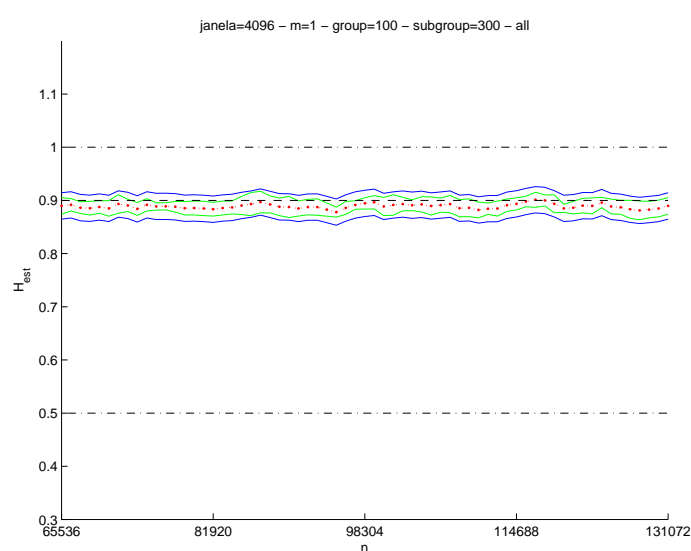

(a) $j_{\min }=1 / N=4096$

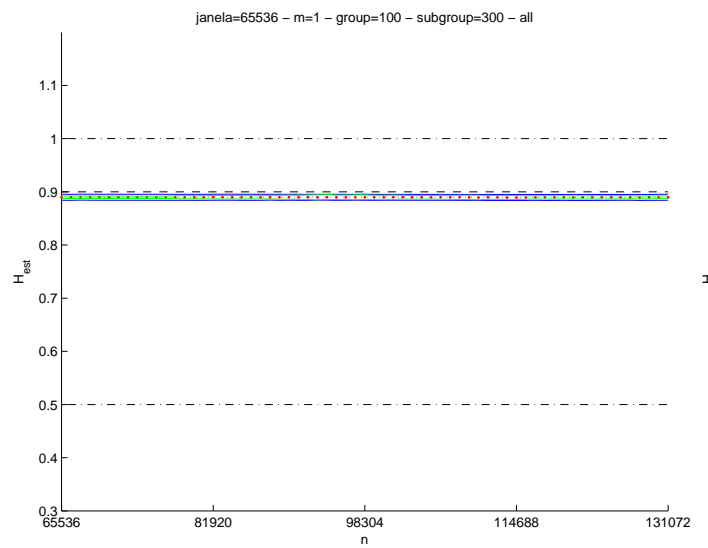

(c) $j_{\min }=1 / N=65536$

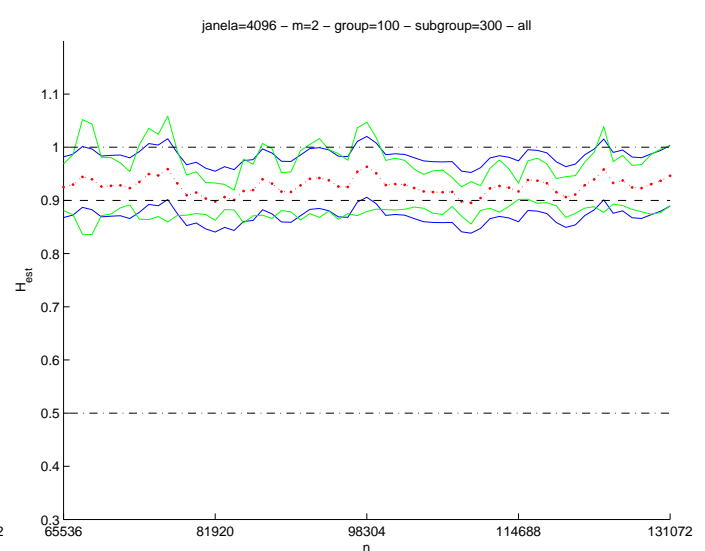

(b) $j_{\min }=j_{\min }^{E Q M} / N=4096$

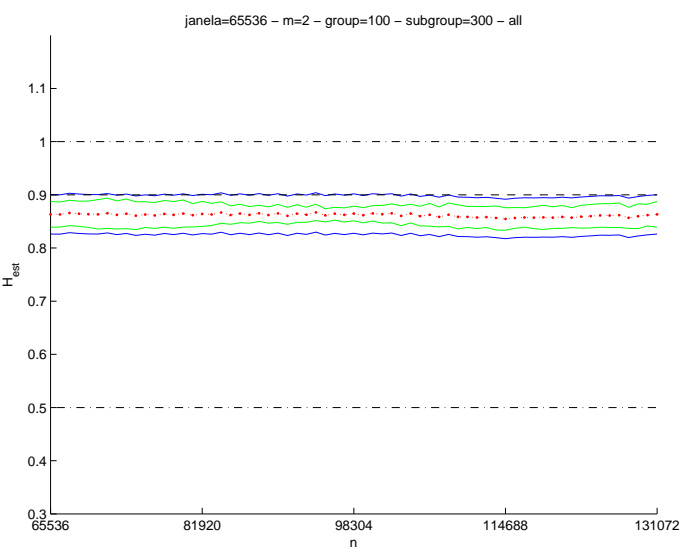

(d) $j_{\min }=j_{\min }^{E Q M} / N=65536$

Figura 6.34: Estimação de séries concatenadas (controle) $H=0,9 ; f_{0}=0 \Longrightarrow H=0,9 ; f_{0}=0$. 
Também para efeitos de controle, foram obtidas as estimativas para o caso de séries com valores de $H$ distintos, mas sem componentes SRD, representadas nas Figuras 6.35 e 6.36. É possível observar em tais Figuras que as estimativas têm uma região de transição entre o regime anterior e o novo regime, sendo que tal região tem tamanho igual ao número de amostras da janela utilizada. Desta maneira, para as estimativas com janela de 4096 amostras, a transição é relativamente rápida, embora a dispersão seja relativamente alta. Para o caso de janela de 65536, a transição é relativamente lenta, mas é compensada por uma dispersão das estimativas consideravelmente reduzida. É digno de nota que, principalmente para os casos em que a janela é igual a 65536 amostras, a média das estimativas na região de transição tem um comportamento visualmente semelhante a uma exponencial crescente ou decrescente, dependendo do caso. 


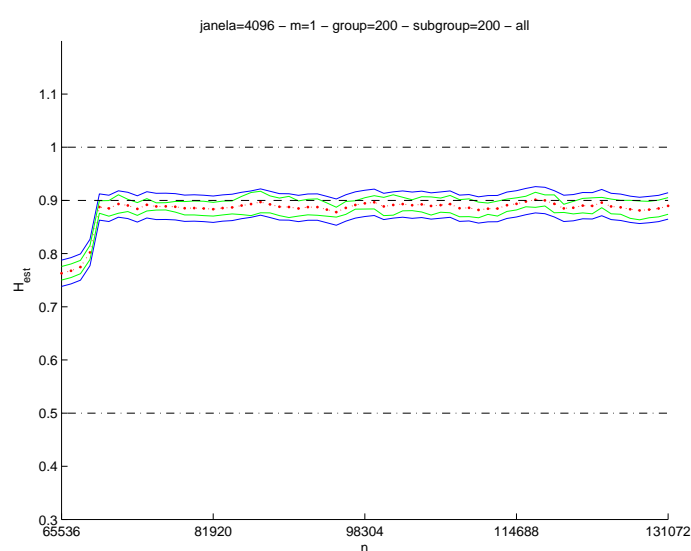

(a) $j_{\min }=1 / N=4096$

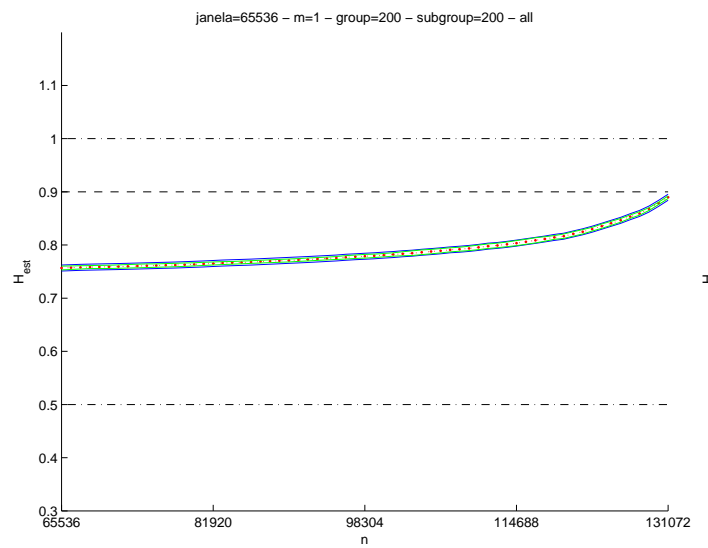

(c) $j_{\min }=1 / N=65536$

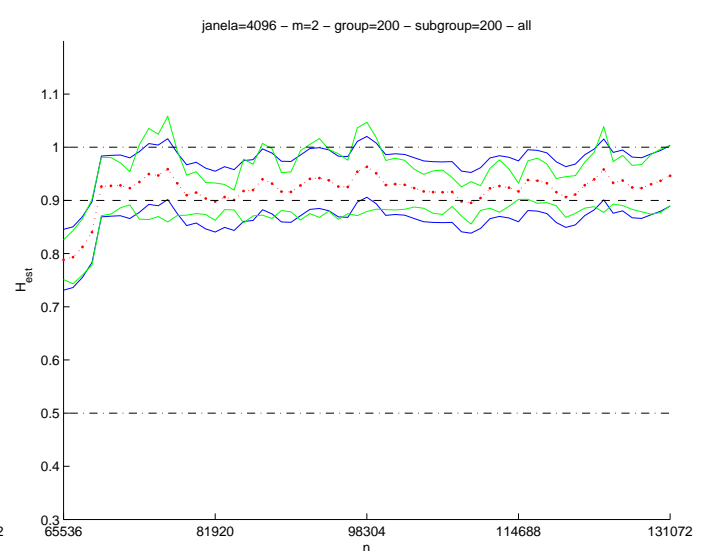

(b) $j_{\min }=j_{\min }^{E Q M} / N=4096$

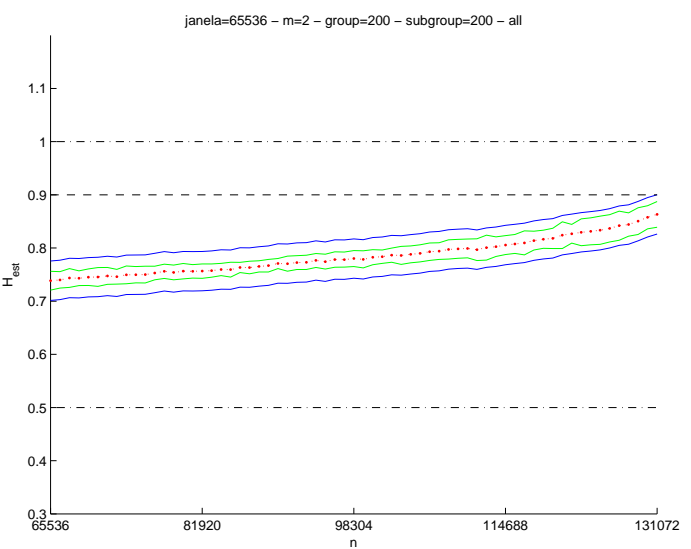

(d) $j_{\min }=j_{\min }^{E Q M} / N=65536$

Figura 6.35: Estimação de séries concatenadas (controle) -

$$
H=0,6 ; f_{0}=0 \Longrightarrow H=0,9 ; f_{0}=0 \text {. }
$$




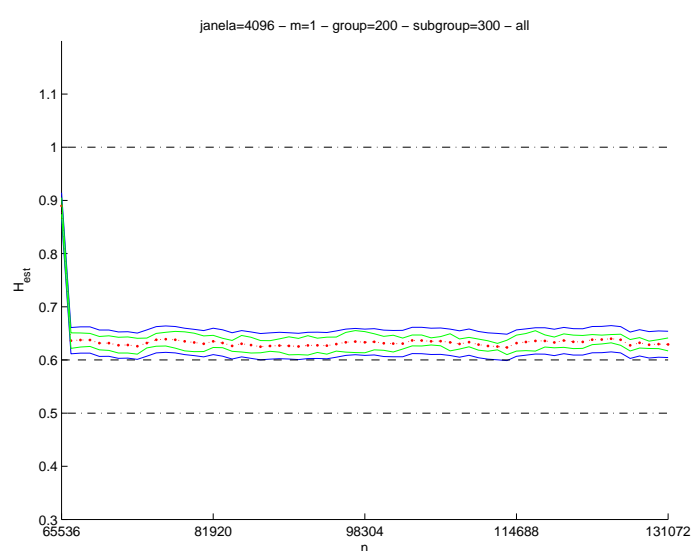

(a) $j_{\min }=1 / N=4096$

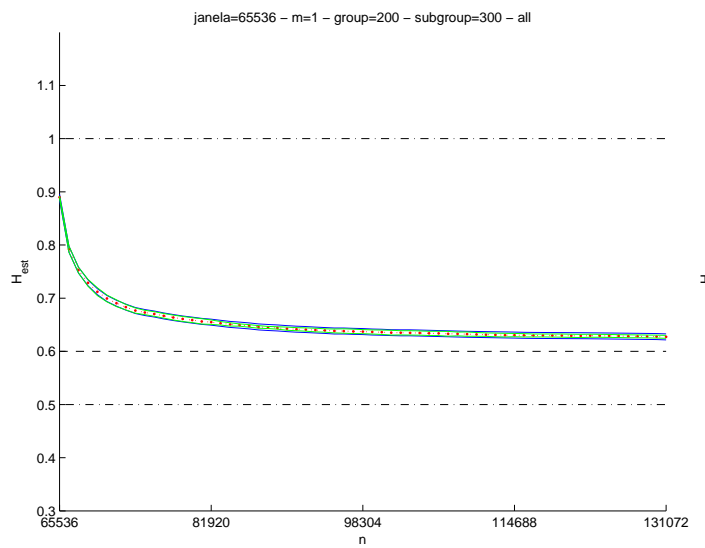

(c) $j_{\min }=1 / N=65536$

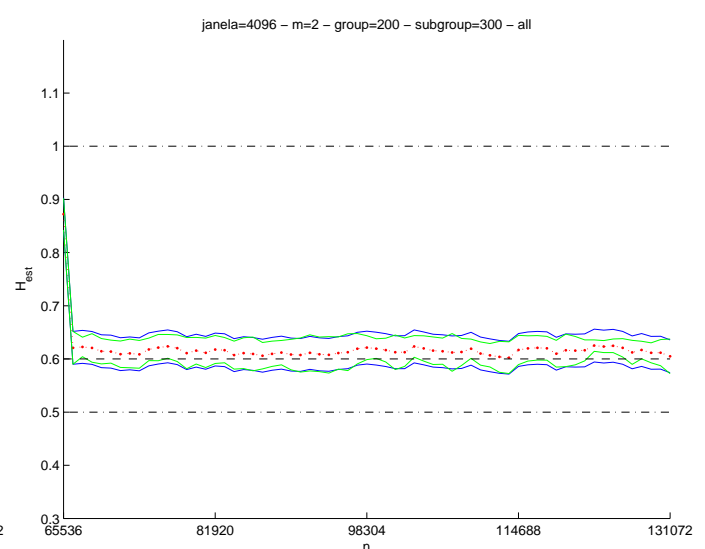

(b) $j_{\min }=j_{\min }^{E Q M} / N=4096$

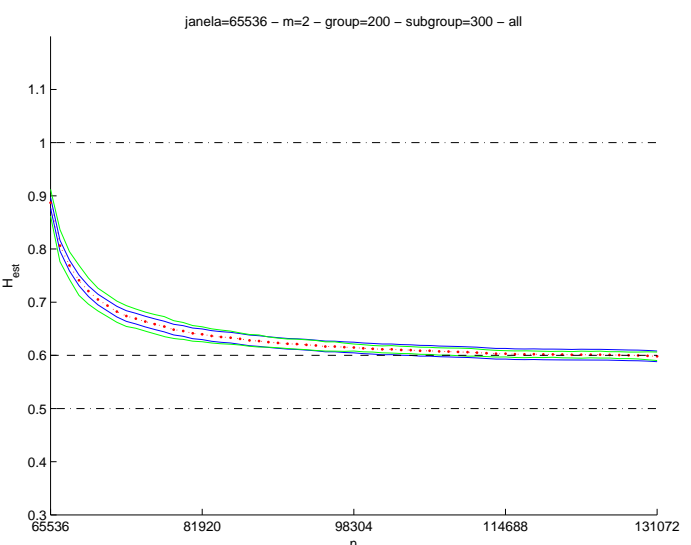

(d) $j_{\min }=j_{\min }^{E Q M} / N=65536$

Figura 6.36: Estimação de séries concatenadas (controle) $H=0,9 ; f_{0}=0 \Longrightarrow H=0,6 ; f_{0}=0$. 
Finalmente, as Figuras 6.37-6.41 representam as estimativas para o caso de séries concatenadas de mesmo valor de $H$, mas variando-se a componente SRD. Também nessas Figuras pode-se observar o mesmo comportamento de transição observado para o caso de séries concatenadas com valores de $H$ distintos. Uma importante diferença, entretanto, diz respeito a tal comportamento, que para o caso em estudo assemelha-se visualmente a uma transição aproximadamente linear, ao contrário do caso em que havia variação de $H$. Tal observação pode ser utilizada para um eventual estudo mais aprofundado de identificação de mudanças de regime da componente SRD.

Também para efeitos de averiguação de mudança de regime, o comportamento observados em tais gráficos durante o regime transitório sugere um possível esquema de estimação que leve em consideração mudanças de regime da componente SRD. Segundo tal esquema, seria possível realizar estimações de forma paralela, por exemplo, com janelas de 4096 e 65536 amostras. As estimações com a janela de 65536 amostras, por exemplo, poderiam ser utilizadas como estimativas com menor viés e dispersão, enquanto as estimações com a janela de 4096 amostras, por exemplo, poderiam ser utilizadas para identificar de forma rápida uma alteração de regime (por exemplo, quando a estimativa ultrapassar determinado valor pré-estabelecido de variação). As estimativas a partir do ponto em que foi identificada a mudança de regime seriam feitas através de uma janela crescente até fosse atingido novamente o valor de 65536 amostras. 


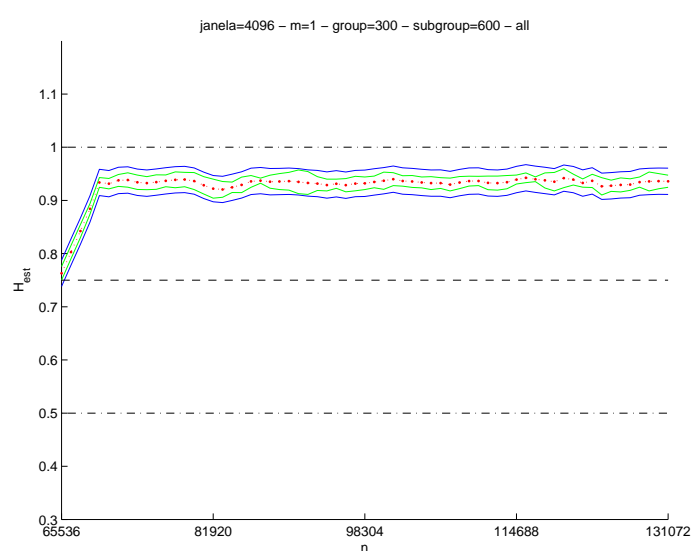

(a) $j_{\min }=1 / N=4096$

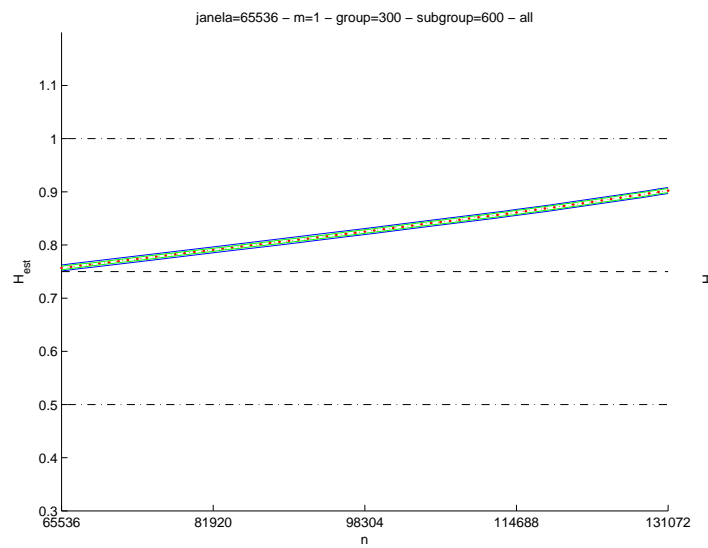

(c) $j_{\min }=1 / N=65536$

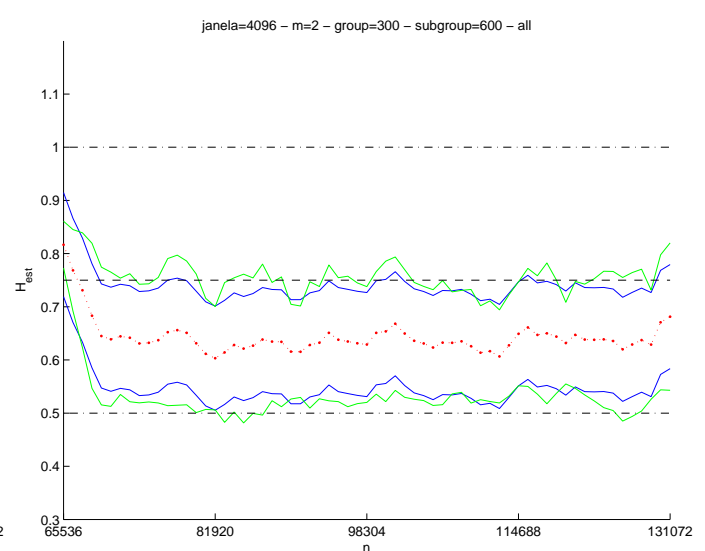

(b) $j_{\min }=j_{\min }^{E Q M} / N=4096$

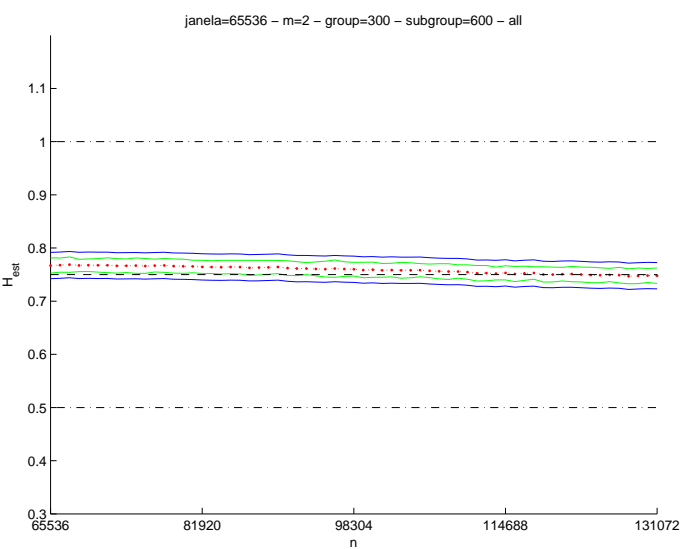

(d) $j_{\text {min }}=j_{\min }^{E Q M} / N=65536$

Figura 6.37: Estimação de séries concatenadas $H=0,75 ; f_{0}=0 \Longrightarrow H=0,75 ; f_{0}=0,2$. 


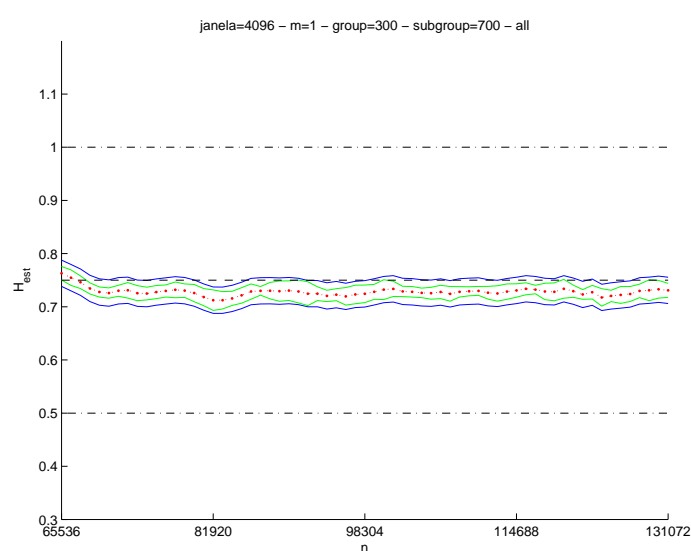

(a) $j_{\min }=1 / N=4096$

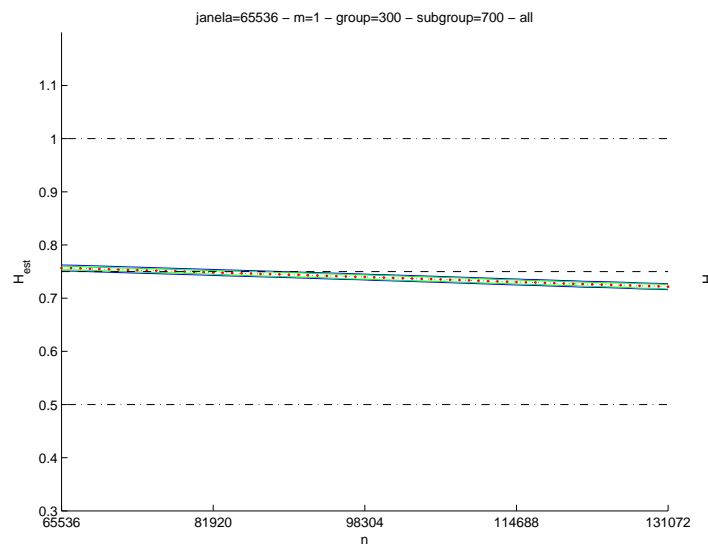

(c) $j_{\min }=1 / N=65536$

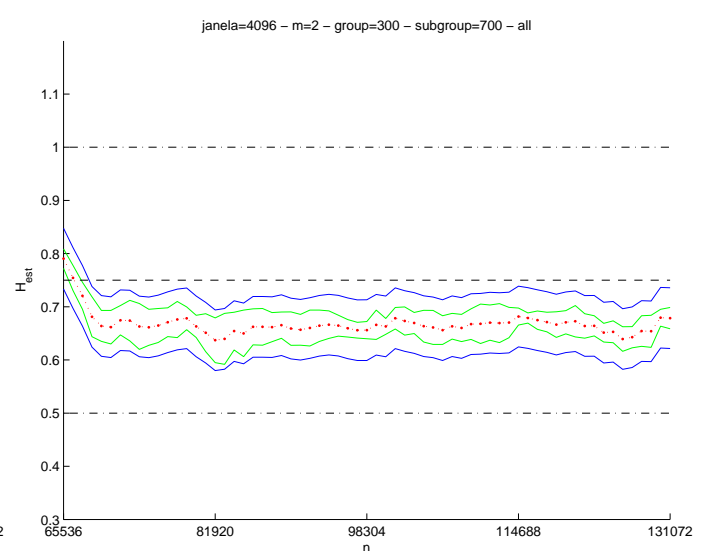

(b) $j_{\min }=j_{\min }^{E Q M} / N=4096$

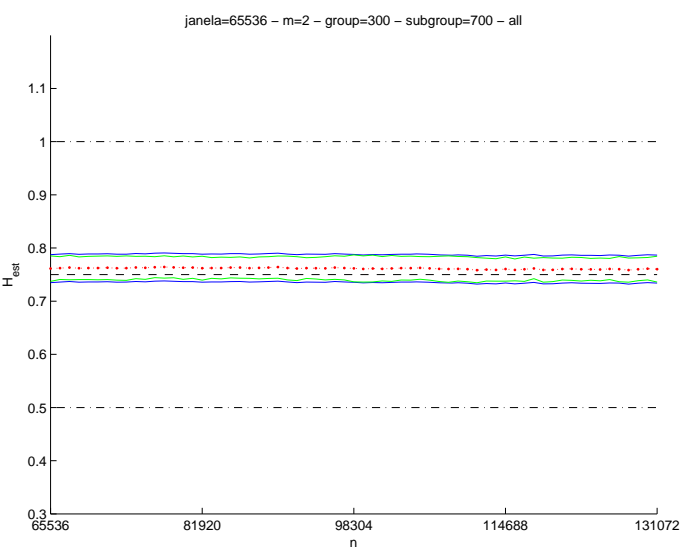

(d) $j_{\text {min }}=j_{\min }^{E Q M} / N=65536$

Figura 6.38: Estimação de séries concatenadas $H=0,75 ; f_{0}=0 \Longrightarrow H=0,75 ; f_{0}=0,4$. 


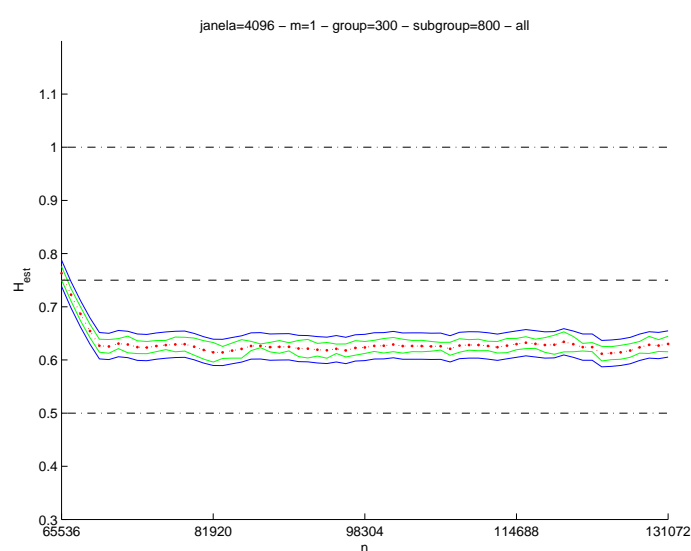

(a) $j_{\min }=1 / N=4096$

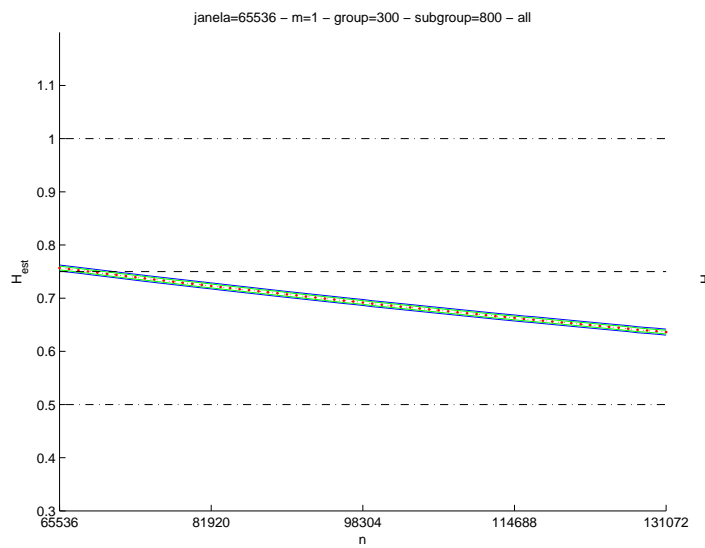

(c) $j_{\min }=1 / N=65536$

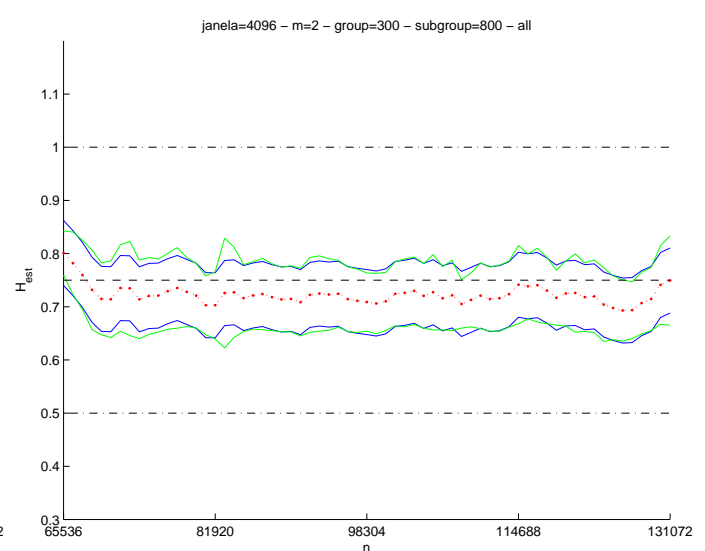

(b) $j_{\min }=j_{\min }^{E Q M} / N=4096$

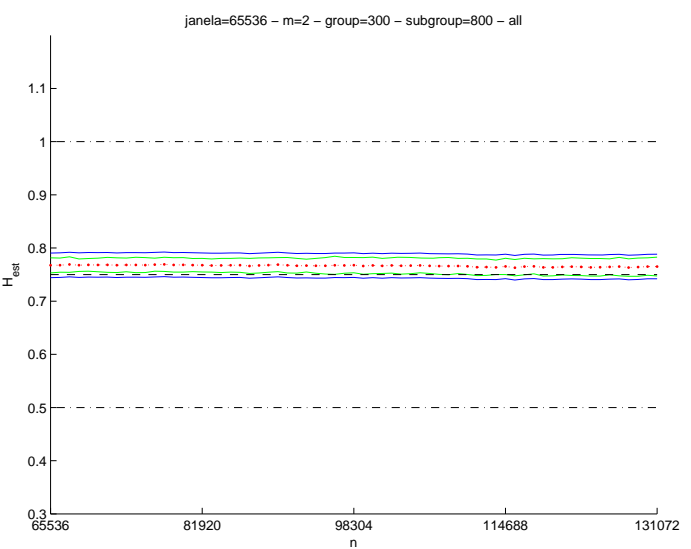

(d) $j_{\text {min }}=j_{\min }^{E Q M} / N=65536$

Figura 6.39: Estimação de séries concatenadas $H=0,75 ; f_{0}=0 \Longrightarrow H=0,75 ; f_{0}=0,7$. 


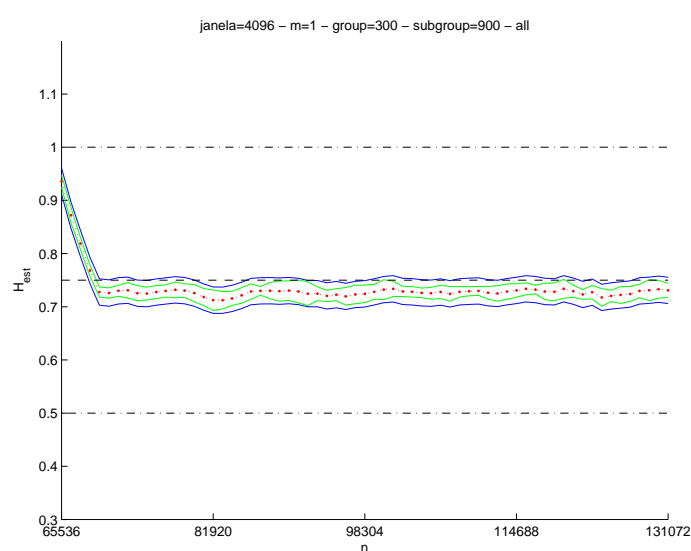

(a) $j_{\min }=1 / N=4096$

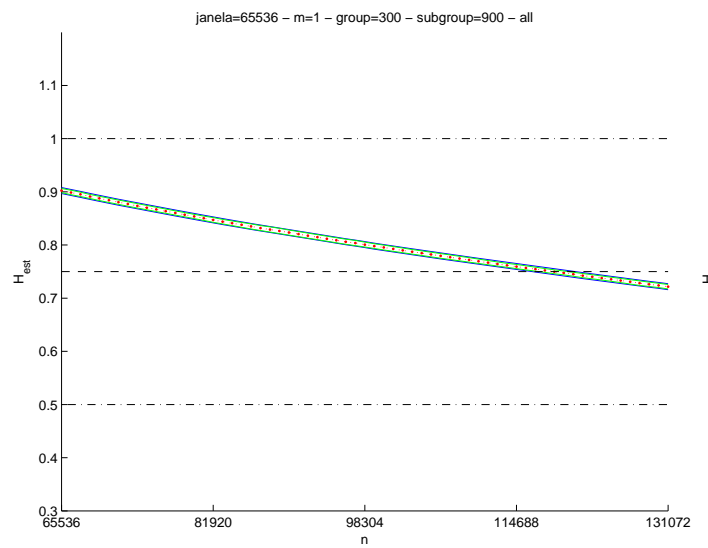

(c) $j_{\text {min }}=1 / N=65536$

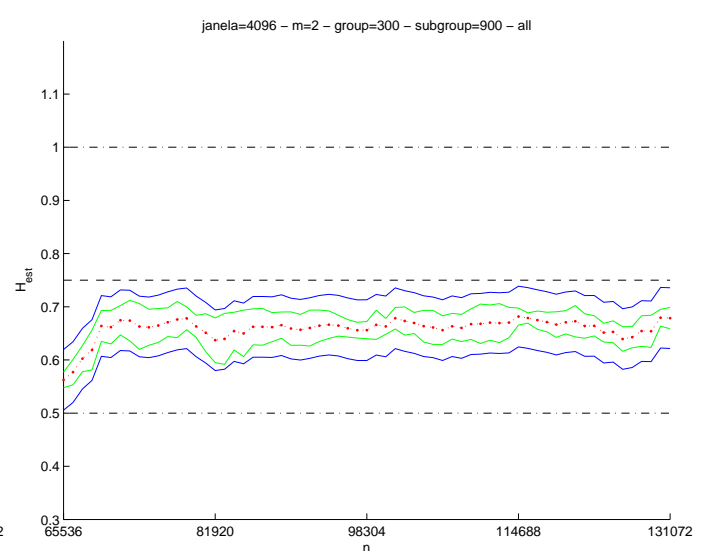

(b) $j_{\min }=j_{\min }^{E Q M} / N=4096$

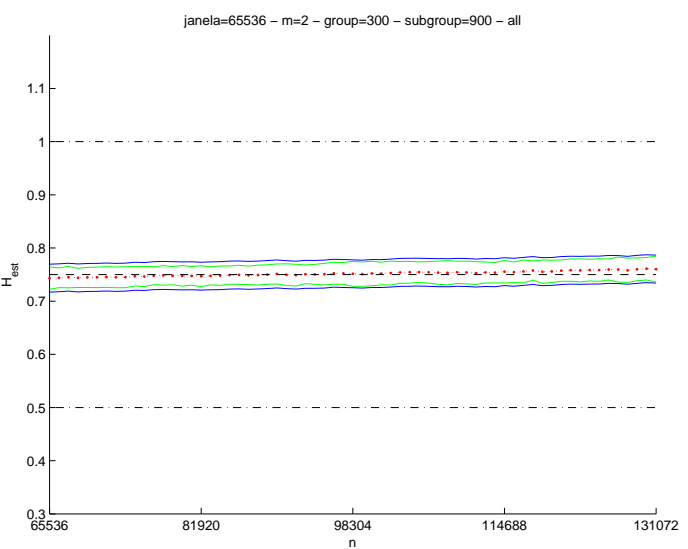

(d) $j_{\text {min }}=j_{\min }^{E Q M} / N=65536$

Figura 6.40: Estimação de séries concatenadas $H=0,75 ; f_{0}=0,2 \Longrightarrow H=0,75 ; f_{0}=0,4$. 


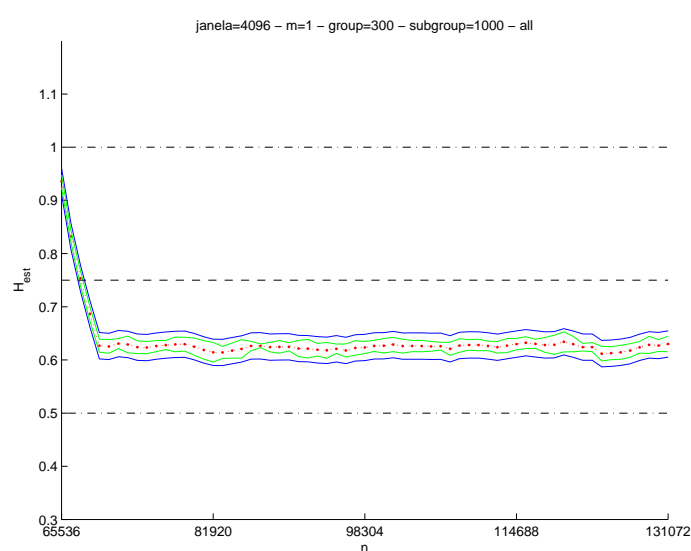

(a) $j_{\min }=1 / N=4096$

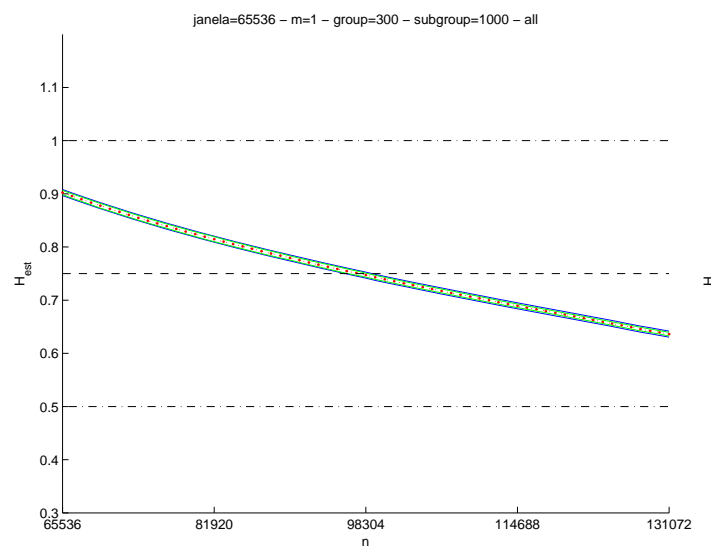

(c) $j_{\text {min }}=1 / N=65536$

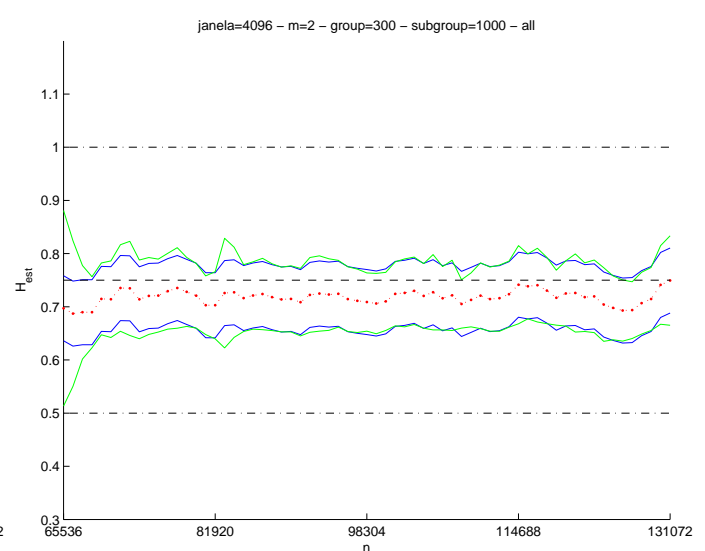

(b) $j_{\min }=j_{\min }^{E Q M} / N=4096$

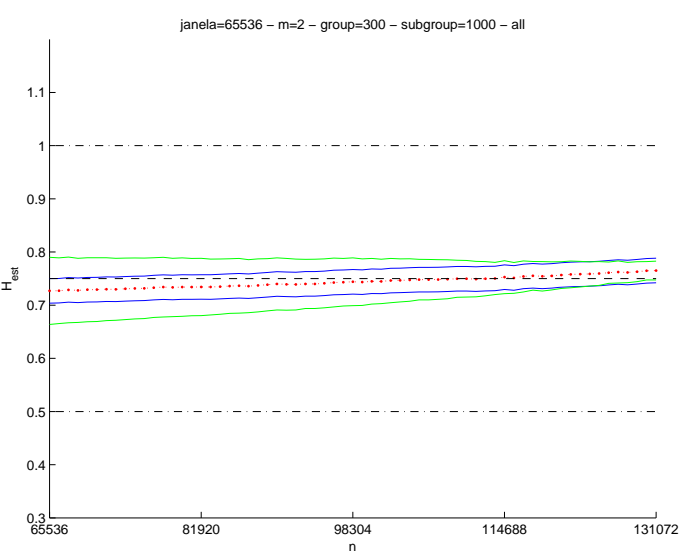

(d) $j_{\text {min }}=j_{\min }^{E Q M} / N=65536$

Figura 6.41: Estimação de séries concatenadas $H=0,75 ; f_{0}=0,2 \Longrightarrow H=0,75 ; f_{0}=0,7$. 


\subsubsection{Exploração dos resultados}

Para melhor compreender os resultados apresentados na Seção 6.3.2, em particular os diferentes comportamentos de transição, tanto para o caso de transição de valor de $H$ como para o caso de transição de componente SRD, são apresentados nessa Seção os espectros wavelet médios, entre todas as realizações disponíveis com a característica indicada.

A Figura 6.42 apresenta inicialmente os espectros para o caso de transição no nível de $H$, tanto para o caso de janela de estimação com $N=4096$ (Figura 6.42a) como para o caso de janela de estimação com $N=65536$ (Figura 6.42b). Mais uma vez, o gráfico correspondente a $N=65536$ melhor apresenta visualmente o comportamento de transição. Conforme ilustrado na Figura 6.42b, é possível observar que: (a) a transição do espectro não ocorre em apenas algumas escalas, mas pelo menos nas dez escalas inferiores; (b) em todas as escalas afetadas, mas de forma mais notória nas escalas inferiores, ocorre um efeito análogo, mas mais pronunciado do que observado na transição das estimativas: a região de transição para uma dada escala fixa é tal que a transição em tal escala observa um comportamento exponencial.

De forma análoga, são apresentados também os espectros wavelet para os casos de transição da componente SRD, conforme figuras 6.43 e 6.44. De forma distinta do observado na Figura 6.42, a transição da componente SRD não é observada em um grande número de escalas, mas apenas nas 5 escalas inferiores do espectro, o que está de acordo com os resultados anteriormente observados de que a região influenciada pelas componentes SRD é limitada. Adicionalmente, nas escalas em que existe transição, tal transição não apresenta o comportamento exponencial da Figura 6.42, mas aproximadamente linear, também de acordo com o comportamento observado nas estimativas de $H$ obtidas.

Tais resultados permitem que seja idealizado um esquema de estimação tal que seja possível identificar comportamentos de transição e adequadamente associar se tal transição deve-se a uma efetiva mudança no valor de $H$ da série em questão, ou se trata-se de uma mudança nas componentes SRD que acabam por resultar em uma pseudoalteração na estimativa de $H$. 


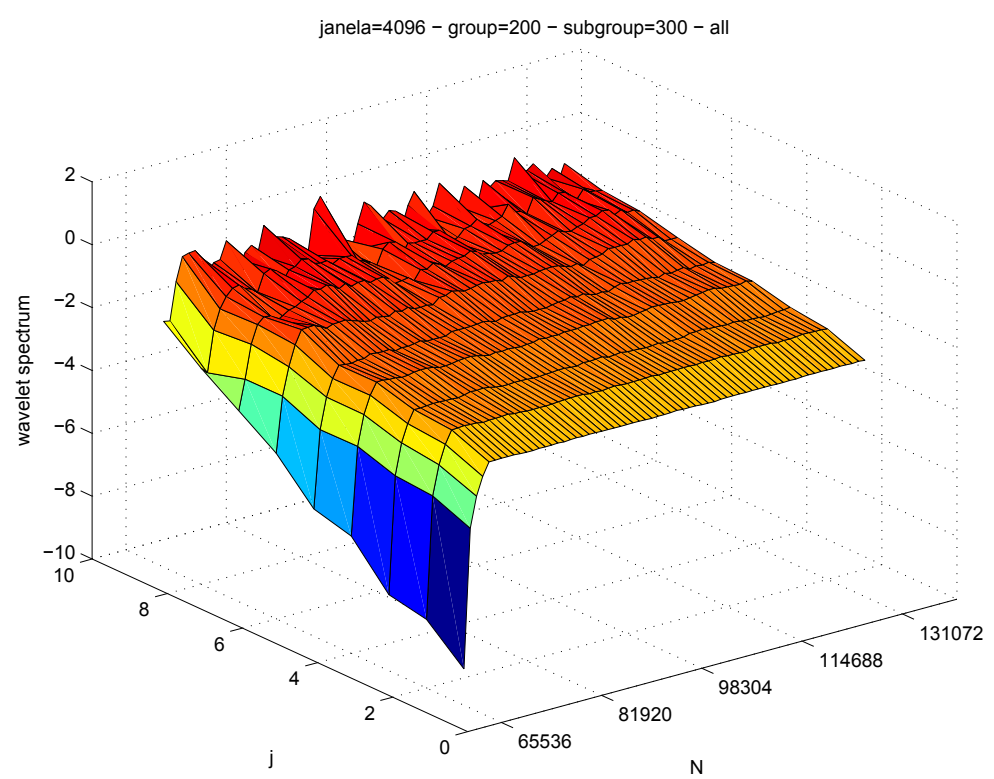

(a) Janela de estimação com $N=4096$

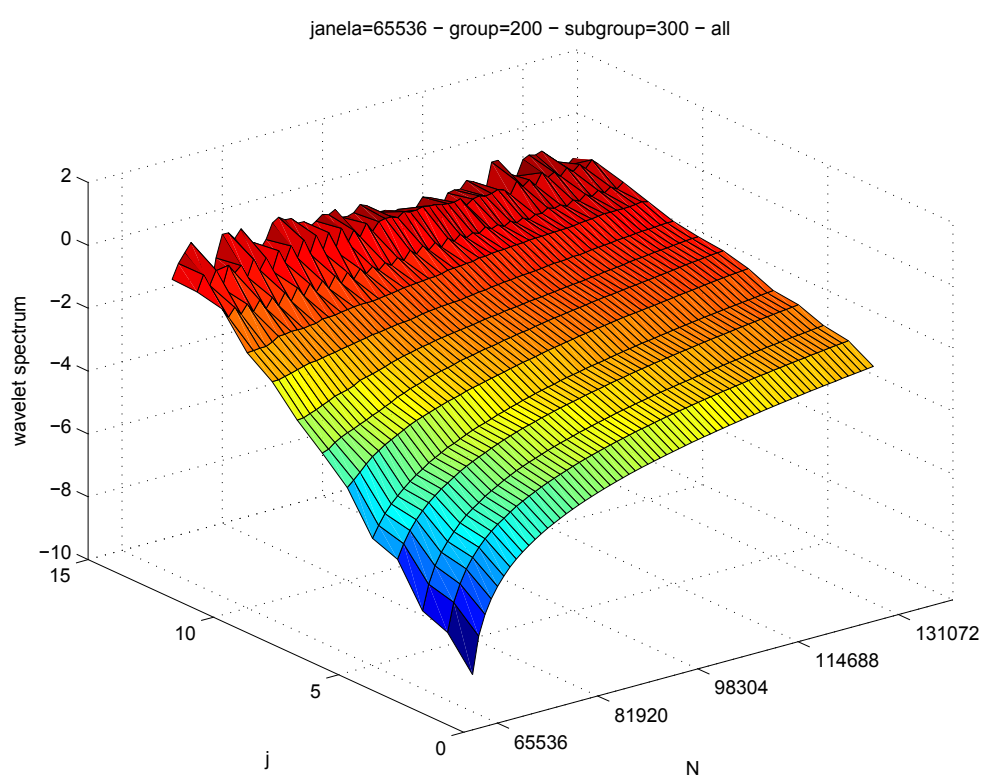

(b) Janela de estimação com $N=65536$

Figura 6.42: Espectro wavelet de séries concatenadas $H=0,9 ; f_{0}=0 \Longrightarrow H=0,6 ; f_{0}=0$. 


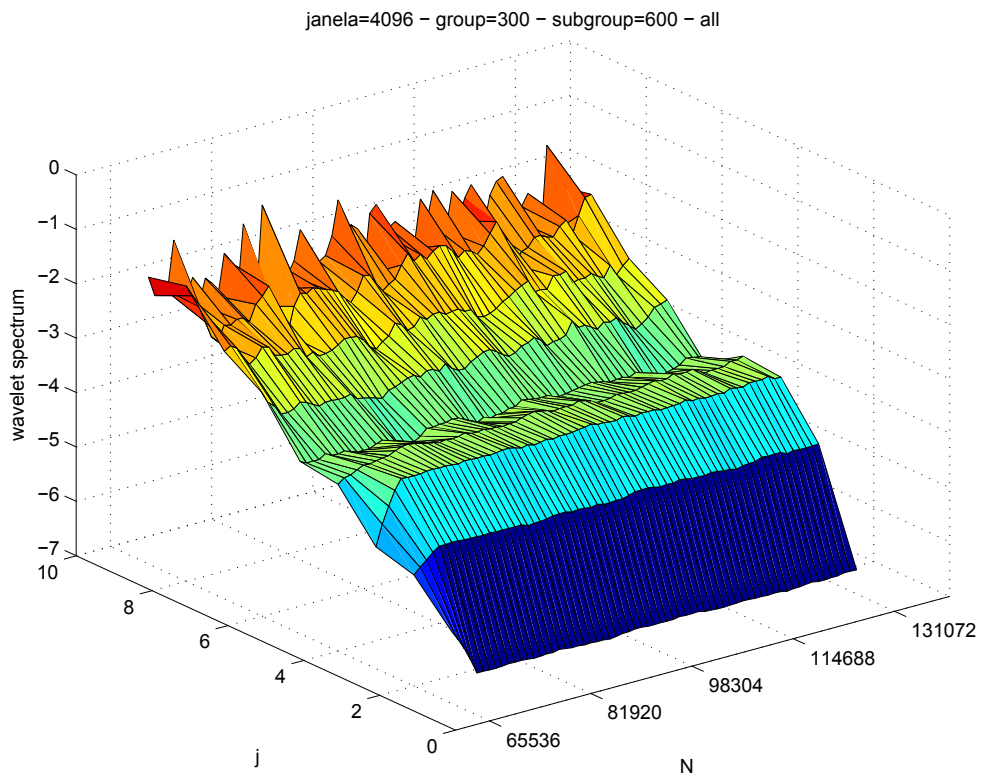

(a) Janela de estimação com $N=4096$

janela $=65536-$ group $=300-$ subgroup $=600-$ all

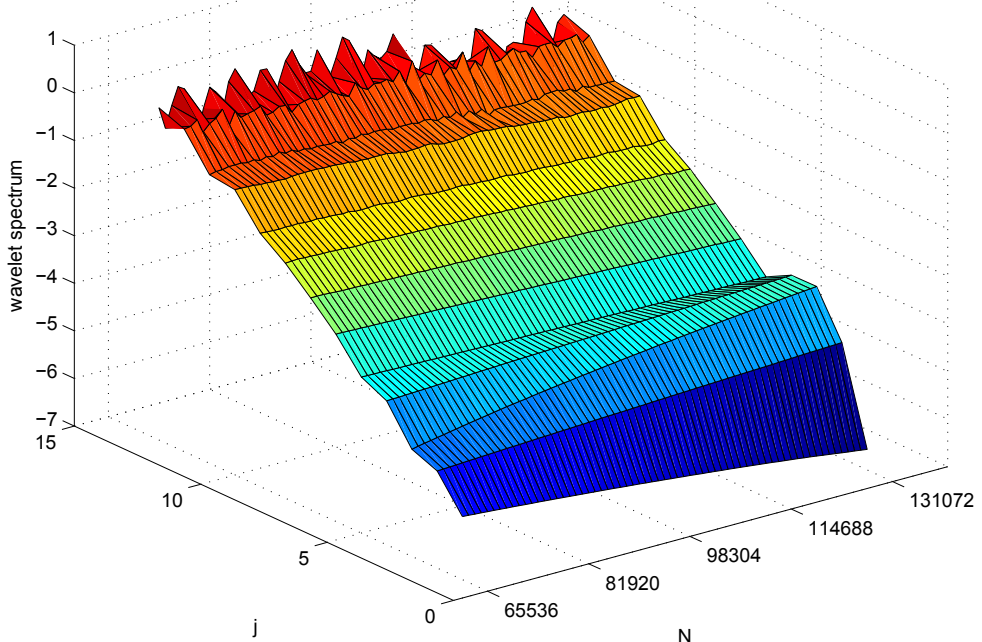

(b) Janela de estimação com $N=65536$

Figura 6.43: Espectro wavelet de séries concatenadas $H=0,75 ; f_{0}=0 \Longrightarrow H=0,75 ; f_{0}=0,2$. 


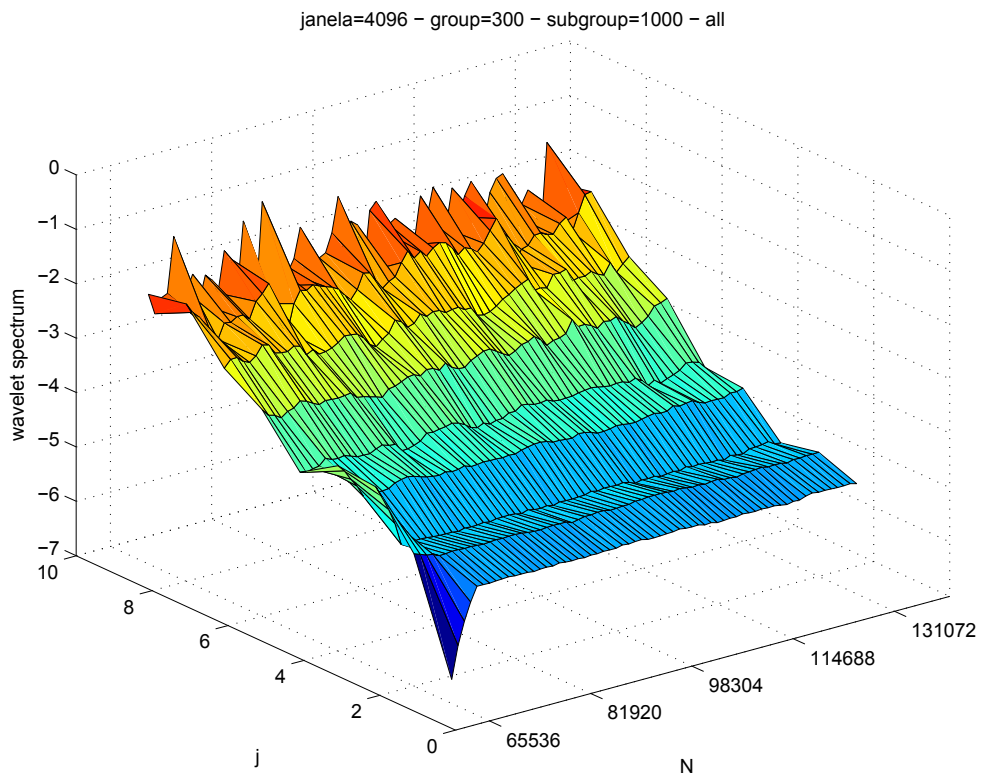

(a) Janela de estimação com $N=4096$

janela $=65536-$ group $=300-$ subgroup $=1000-$ all

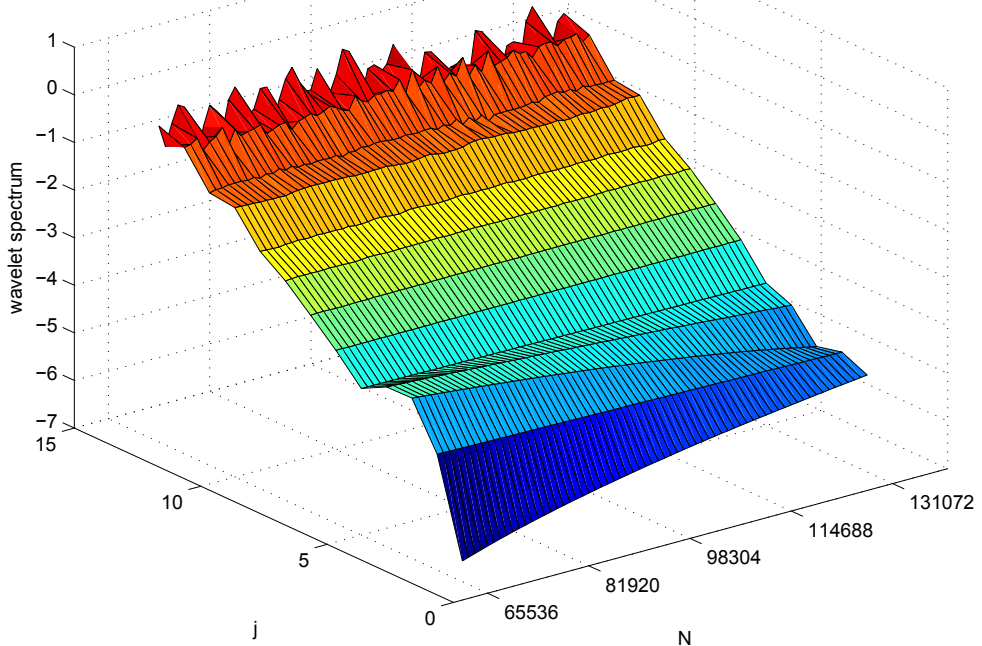

(b) Janela de estimação com $N=65536$

Figura 6.44: Espectro wavelet de séries concatenadas $H=0,75 ; f_{0}=0,2 \Longrightarrow H=0,75 ; f_{0}=0,7$. 


\subsubsection{Síntese dos resultados}

A Tabela 6.3 apresenta uma síntese dos principais resultados expostos na Seção 6.3 .

Tabela 6.3: Resultados da Seção 6.3.

\begin{tabular}{|c|l|}
\hline \hline Resultado & Descrição \\
\hline \hline 1 & $\begin{array}{l}\text { Comportamento transitório, tanto no caso de mudança de nível de } H \text { como de } \\
\text { mudança na componente SRD tem duração proporcional ao tamanho da janela } \\
(N) \text { utilizada na estimação }\end{array}$ \\
\hline 2 & $\begin{array}{l}\text { Nível da dispersão das estimativas tem comportamento inversamente propor- } \\
\text { cional ao tamanho da janela de estimação }(N) \text { utilizada }\end{array}$ \\
\hline 3 & $\begin{array}{l}\text { Proposto mecanismo de estimação que combine a utilização da maior janela de } \\
\text { estimação possível (para redução da dispersão das estimativas) com a utilização } \\
\text { da menor janela de estimação possível (para rápida detecção de transições no } \\
\text { nível de } H \text { ou da componente SRD) }\end{array}$ \\
\hline 4 & $\begin{array}{l}\text { No caso de transição do valor de H, tanto as estimativas de } H \text { como as escalas } \\
\text { do espectro wavelet alteradas na transição têm comportamento exponencial. } \\
\text { Um grande número de escalas é afetado por essa transição }\end{array}$ \\
\hline 5 & $\begin{array}{l}\text { No caso de transição da componente SRD, tanto as estimativas de } H \text { como as } \\
\text { escalas do espectro wavelet alteradas na transição têm comportamento linear. } \\
\text { Um número reduzido de escalas é afetado por essa transição }\end{array}$ \\
\hline \hline
\end{tabular}




\subsection{Análise comparativa detalhada com resulta- dos obtidos por Abry-Veitch}

Os resultados apresentados até o momento nas Seções anteriores caracterizam-se por serem mais qualitativos do que quantitativos. O artigo [10] de Abry traz, nas páginas 20-22, resultados quantitativos teóricos e empíricos bastante detalhados de estimativas utilizando tanto um modelo exclusivamente LRD (no caso, o modelo FGN) como um modelo que inclui componentes SRD (no caso, o modelo FARIMA). A Tabela 6.4 resume os resultados de interesse de tal artigo.

Como no presente trabalho o estudo de séries com SRD não foi feito através do modelo FARIMA, mostra-se necessária a comparação dos resultados de tal modelo com os do modelo SRD utilizado nesta dissertação.

\subsubsection{Condições experimentais}

Os resultados empíricos obtidos no artigo [10] (vide Tabela 6.4, com a compilação da Tabela 1 - resultado teórico - e da Tabela 2 - resultado empírico - desse artigo) foram obtidos através de simulações com 50 realizações para cada condição do modelo em estudo, sendo que foram utilizados tanto o modelo FGN como diversas combinações do modelo FARIMA. Em todos os casos foi observado o comportamento para valores de $H$ iniciando-se em 0,5 , com incrementos de 0,1 ; adicionalmente, $j_{\min }^{E Q M}$ foi fixado para cada combinação de parâmetros do modelo, não sendo variável para cada realização.

No presente trabalho, as condições acima foram expandidas de forma que as séries estudadas foram geradas de acordo com as seguintes condições:

- $H_{\text {gen }}$ : de 0,50 a 0,95 , com incrementos de 0,05

- Número de realizações para cada combinação de parâmetros dos modelos: 128

- Modelo de geração LRD: FGN

- Modelo de geração SRD: filtragem IIR tal que $f_{0}$ varie de 0,1 a 0,9, com incrementos de 0,1

- Amostras por realização: $N=2^{n}$, onde $n=8,10, \ldots, 14,16$

- $j_{\min } \in\left\{1, j_{\min }^{E Q M}\right\}$ (sendo que $j_{\min }^{E Q M}$ é variável para cada realização). 


\subsubsection{Resultados obtidos}

Após a geração das estimações de $H$ para todas as condições descritas na Seção 6.4.1, os resultados foram compilados em uma tabela com as 900 combinações dos três parâmetros $H_{g e n}, N$ e 'modelo de geração'. Para cada uma destas combinações, foram obtidas as seguintes variáveis:

- valor médio, desvio padrão e moda de $j_{\min }^{E Q M}$

- desvio padrão e viés da estimativa de $H$ utilizando-se de $j_{\min }=1$

- desvio padrão e viés da estimativa de $H$ utilizando-se de $j_{\min }=j_{\min }^{E Q M}$

A Tabela 6.5 apresenta um resumo dos resultados obtidos. Deve-se observar que, para efeitos de comparação com os resultados da Tabela 6.4, são consideradas apenas as realizações tal que:

- $H_{\text {gen }}$ : de 0,5 a 0,9 , com incrementos de 0,1

- Amostras por realização: $N=16384$

- Modelo de geração LRD: FGN

- Modelo de geração SRD: filtragem IIR tal que $f_{0}$ varie de 0,2 a 0,8 , com incrementos de 0,2

Conforme pode ser observado através da comparação das Tabelas 6.4 e 6.5, não é possível uma comparação direta, linha a linha, entre tais Tabelas, uma vez que apenas o modelo FGN é comum a ambas e, mesmo assim, enquanto na Tabela 6.4 as estimações são feitas com $j_{\min }$ fixo para todas as realizações, na Tabela 6.5 as estimações foram realizadas com $j_{\min }$ variável para cada realização. De qualquer maneira, a observação da coluna com os valores estimados de $H$ da Tabela 6.4 com a coluna com os valores estimados de $H$ tal que $j_{\text {min }}=j_{\text {min }}^{E Q M}$ demonstra que tanto a média quanto a variância do estimador em ambos os casos têm, em módulo, a mesma ordem de grandeza, indicando coerência nos resultados apresentados. 
Tabela 6.4: Compilação dos resultados teórico e empírico para estimação de $H$, segundo Abry, para modelos FGN e FARIMA.

\begin{tabular}{|c|c|c|c|c|c|c|c|}
\hline \multirow[b]{2}{*}{ Modelo } & \multirow[b]{2}{*}{$H$} & \multicolumn{3}{|c|}{ Teórico } & \multicolumn{3}{|c|}{ Estimado } \\
\hline & & $j_{\min }^{E Q M}$ & Viés & 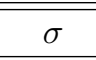 & $j_{\min }^{E Q M}$ & Viés & $\bar{\sigma} \sigma$ \\
\hline \multirow[t]{5}{*}{ fGn } & $\overline{0,5}$ & 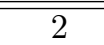 & ב-0,004 & 0,011 & 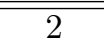 & $2-0,005$ & $\overline{c 0,013}$ \\
\hline & 0,6 & 2 & $-0,010$ & 0,011 & 2 & $-0,013$ & 0,013 \\
\hline & 0,7 & 3 & $-0,005$ & 0,017 & 3 & $-0,006$ & 0,017 \\
\hline & 0,8 & 3 & $-0,005$ & 0,017 & 3 & $-0,002$ & 0,020 \\
\hline & 0,9 & 3 & $-0,006$ & 0,017 & 3 & $-0,004$ & 0,016 \\
\hline \multirow[t]{4}{*}{ farima $(0, d, 0)$} & 0,6 & 2 & 0,000 & 0,011 & 2 & 0,000 & 0,013 \\
\hline & 0,7 & 2 & 0,003 & 0,011 & 2 & 0,002 & 0,010 \\
\hline & 0,8 & 1 & $-0,008$ & 0,008 & 1 & $-0,009$ & 0,008 \\
\hline & 0,9 & 1 & 0,002 & 0,008 & 1 & 0,006 & 0,008 \\
\hline farima $(1, \mathrm{~d}, 0)$ & 0,5 & 5 & $\begin{array}{c}-0,014 \\
\end{array}$ & 0,040 & 5 & 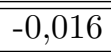 & 0,038 \\
\hline \multirow[t]{4}{*}{$\phi_{1}=0,5$} & 0,6 & 5 & $-0,013$ & 0,040 & 5 & $-0,016$ & 0,033 \\
\hline & 0,7 & 5 & $-0,013$ & 0,040 & 5 & $-0,010$ & 0,038 \\
\hline & 0,8 & 5 & $-0,012$ & 0,040 & 5 & $-0,015$ & 0,042 \\
\hline & 0,9 & 5 & $-0,011$ & 0,040 & 5 & $-0,022$ & 0,043 \\
\hline farima $(0, \mathrm{~d}, 1)$ & 0,5 & 5 & 0,016 & 0,040 & 5 & 0,014 & "0,039 \\
\hline \multirow[t]{4}{*}{$\theta_{1}=0,5$} & 0,6 & 5 & 0,015 & 0,040 & 5 & 0,010 & 0,038 \\
\hline & 0,7 & 5 & 0,014 & 0,040 & 5 & 0,020 & 0,040 \\
\hline & 0,8 & 5 & 0,013 & 0,040 & 5 & 0,011 & 0,040 \\
\hline & 0,9 & 5 & 0,012 & 0,040 & 5 & 0,019 & 0,036 \\
\hline farima $(1, \mathrm{~d}, 1)$ & $\overline{0,5}$ & $\overline{5}$ & 0,049 & $\overline{00,040}$ & 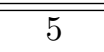 & $\overline{00,050}$ & $\overline{0,054}$ \\
\hline$\phi_{1}=0,3$ & 0,6 & 5 & 0,046 & 0,040 & 5 & 0,038 & 0,043 \\
\hline \multirow[t]{3}{*}{$\theta_{1}=0,7$} & 0,7 & 5 & 0,044 & 0,040 & 6 & 0,006 & 0,063 \\
\hline & 0,8 & 5 & 0,041 & 0,040 & 5 & 0,035 & 0,045 \\
\hline & 0,9 & 5 & 0,039 & 0,040 & 5 & 0,037 & 0,046 \\
\hline farima $(1, \mathrm{~d}, 1)$ & $\overline{0,5}$ & $\overline{3}$ & "-0,008 & 0,017 & $\overline{3}$ & $\begin{array}{l}-0,007 \\
\end{array}$ & 0,017 \\
\hline$\phi_{1}=-0,3$ & 0,6 & 3 & $-0,007$ & 0,017 & 3 & $-0,004$ & 0,018 \\
\hline \multirow[t]{3}{*}{$\theta_{1}=-0,7$} & 0,7 & 3 & $-0,005$ & 0,017 & 3 & $-0,008$ & 0,016 \\
\hline & 0,8 & 3 & $-0,004$ & 0,017 & 3 & $-0,005$ & 0,017 \\
\hline & 0,9 & 3 & $-0,003$ & 0,017 & 3 & $-0,005$ & 0,017 \\
\hline farima $(1, \mathrm{~d}, 1)$ & $\overline{0,5}$ & $\overline{5}$ & \begin{tabular}{c|}
$-0,043$ \\
\end{tabular} & 0,040 & $\overline{5}$ & $2-0,040$ & 0,038 \\
\hline$\phi_{1}=0,7$ & 0,6 & 5 & $-0,041$ & 0,040 & 5 & $-0,050$ & 0,044 \\
\hline \multirow[t]{3}{*}{$\theta_{1}=0,3$} & 0,7 & 5 & $-0,039$ & 0,040 & 6 & $-0,027$ & 0,047 \\
\hline & 0,8 & 5 & $-0,037$ & 0,040 & 5 & $-0,032$ & 0,041 \\
\hline & 0,9 & 5 & $-0,036$ & 0,040 & 5 & $-0,040$ & 0,044 \\
\hline
\end{tabular}


Tabela 6.5: Resultados empíricos quantitativos da estimação de $H$ utilizando-se modelos sem e com componentes SRD.

\begin{tabular}{|c|c||c|c|c||c|c||c|c|}
\hline \hline & \multicolumn{1}{|c||}{} & \multicolumn{3}{c||}{$j_{\min }^{E Q M}$} & \multicolumn{2}{c||}{$H\left(j_{\min }=1\right)$} & \multicolumn{2}{c|}{$H\left(j_{\min }=j_{\min }^{E Q M}\right)$} \\
\hline \hline Modelo & $H$ & Média & $\sigma$ & Moda & Viés & $\sigma$ & Viés & $\sigma$ \\
\hline \hline fGn & 0,5 & 2,00 & 0,00 & 2 & 0,045 & 0,006 & 0,003 & 0,007 \\
\hline & 0,6 & 2,06 & 0,39 & 2 & 0,028 & 0,006 & $-0,006$ & 0,012 \\
\hline & 0,7 & 2,94 & 0,96 & 2 & 0,014 & 0,006 & $-0,003$ & 0,029 \\
\hline & 0,8 & 4,13 & 1,16 & 4 & 0,002 & 0,005 & $-0,004$ & 0,054 \\
\hline & 0,9 & 4,98 & 0,66 & 5 & $-0,010$ & 0,007 & 0,014 & 0,066 \\
\hline \hline $\mathrm{fGn}^{*}$ & 0,5 & 4,62 & 0,52 & 5 & 0,233 & 0,006 & $-0,058$ & 0,046 \\
\hline$f=0,2$ & 0,6 & 4,90 & 0,35 & 5 & 0,203 & 0,006 & $-0,030$ & 0,042 \\
\hline & 0,7 & 4,98 & 0,18 & 5 & 0,176 & 0,006 & $-0,011$ & 0,036 \\
\hline & 0,8 & 5,09 & 0,29 & 5 & 0,150 & 0,006 & 0,001 & 0,047 \\
\hline & 0,9 & 4,98 & 0,80 & 5 & 0,124 & 0,007 & 0,011 & 0,071 \\
\hline \hline $\mathrm{fGn}^{*}$ & 0,5 & 3,86 & 0,37 & 4 & 0,042 & 0,006 & $-0,032$ & 0,029 \\
\hline$f=0,4$ & 0,6 & 3,88 & 0,61 & 4 & 0,013 & 0,006 & $-0,037$ & 0,035 \\
\hline & 0,7 & 4,04 & 0,86 & 4 & $-0,014$ & 0,007 & $-0,029$ & 0,052 \\
\hline & 0,8 & 4,37 & 1,07 & 5 & $-0,039$ & 0,006 & $-0,017$ & 0,062 \\
\hline & 0,9 & 4,21 & 1,17 & 3 & $-0,063$ & 0,007 & $-0,011$ & 0,071 \\
\hline \hline $\mathrm{fGn}^{*}$ & 0,5 & 3,06 & 0,24 & 3 & $-0,072$ & 0,006 & $-0,024$ & 0,017 \\
\hline$f=0,6$ & 0,6 & 3,02 & 0,18 & 3 & $-0,090$ & 0,006 & $-0,025$ & 0,014 \\
\hline & 0,7 & 3,05 & 0,30 & 3 & $-0,104$ & 0,007 & $-0,021$ & 0,014 \\
\hline & 0,8 & 3,56 & 0,98 & 3 & $-0,117$ & 0,006 & $-0,014$ & 0,038 \\
\hline & 0,9 & 4,34 & 1,11 & 5 & $-0,130$ & 0,007 & 0,006 & 0,052 \\
\hline \hline $\mathrm{fGn}^{*}$ & 0,5 & 2,16 & 0,38 & 2 & $-0,072$ & 0,006 & $-0,023$ & 0,014 \\
\hline$f=0,8$ & 0,6 & 2,77 & 0,52 & 3 & $-0,087$ & 0,006 & $-0,013$ & 0,020 \\
\hline & 0,7 & 3,13 & 0,41 & 3 & $-0,098$ & 0,006 & $-0,003$ & 0,020 \\
\hline & 0,8 & 3,84 & 1,10 & 3 & $-0,107$ & 0,005 & $-0,003$ & 0,047 \\
\hline & 0,9 & 4,84 & 0,86 & 5 & $-0,115$ & 0,007 & 0,016 & 0,063 \\
\hline \hline
\end{tabular}




\subsubsection{Exploração dos resultados}

As Figuras da presente Seção são o resultado de uma análise aprofundada dos dados de estimação obtidos conforme condições especificadas na Seção 6.4.1.

As Figuras 6.45-6.48 apresentam um estudo, para alguns modelos de geração e valores de $H_{g e n}$ selecionados, do comportamento da escala mínima EQM $\left(j_{\min }^{E Q M}\right)$ e do viés e variância de $H$ estimado em função de $\mathrm{N}$.

Com relação ao comportamento de $j_{\min }^{E Q M}$ em tais Figuras, observa-se uma aparente correlação entre a derivada segunda da média e pontos de máximo do desvio padrão de $j_{\min }^{E Q M}$. Com relação à média de $j_{\min }^{E Q M}$, também observa-se que o crescimento é monotônico com $N$, sendo que este também atinge valores maiores (para um dado modelo de geração) com o aumento de $H_{\text {gen }}$. Com relação ao desvio padrão de $j_{\min }^{E Q M}$, este em geral está limitado a 1 , sendo observado um ligeiro aumento em seu valor (para um dado modelo de geração) com o aumento de $H_{g e n}$.

Para as estimativas de $H$, o comportamento para estimativas com $j_{\min }=1 \mathrm{e}$ $j_{\min }=j_{\min }^{E Q M}$ são distintos.

No caso das estimativas realizadas com $j_{\min }=1$, foi observado que o viés, além de ser monotonicamente decrescente com o aumento de $N$, é decrescente com o aumento de $H_{\text {gen }}$ (podendo, assim, apresentar viés positivo para $H_{\text {gen }} \approx 0,5 \mathrm{e}$ viés negativo para $H_{\text {gen }} \approx 1$, por exemplo). Ainda com relação ao caso em que $j_{\min }=1$, o desvio padrão das estimativas é monotonicamente decrescente com o aumento de $N$, sendo que visualmente não se observa variação do mesmo, considerando-se o mesmo valor de $N$, para valores de $H_{g e n}$ ou para modelos de geração distintos.

Já para o caso em que $j_{\min }=j_{\min }^{E Q M}$, observa-se que a utilização de tal valor de $j_{\text {min }}$ fornece estimativas com menor viés, especialmente para valores mais altos de $N\left(N \approx 2^{16}\right)$. Por outro lado, a utilização de $j_{\text {min }}^{E Q M}$ fornece estimativas, em geral, com o maior desvio padrão, apresentando, inclusive, aumentos súbitos de desvio padrão em função de $N$.

Os dados apresentados sugerem que, na elaboração de um mecanismo de estimação de $H$ na presença de SRD, é possível realizar uma estimativa inicial $\operatorname{com} j_{\text {min }}=1$ (pois apresenta menor dispersão de estimativas) e então realizar uma correção de viés através dos resultados apresentados nos gráficos disponibilizados. Tal correção poderia ainda ser aprimorada encontrando-se uma forma analítica aproximada para a curva de viés para $j_{\min }=1$ em função de $\mathrm{N}$. 
As Figuras 6.49-6.58 apresentam um estudo, para alguns valores de $N$ e modelos de geração selecionados, do comportamento da escala mínima $\operatorname{EQM}\left(j_{\min }^{E Q M}\right)$ e do viés e variância de $\hat{H}$ em função de $H_{g e n}$.

Com relação ao comportamento de $j_{\min }^{E Q M}$ em tais Figuras, não é possível depreender outras conclusões além das já apresentadas para os gráficos em função de $N$.

Por outro lado, a análise das Figuras acima citadas permite confirmar o resultado já apresentado de que, para o caso em que $j_{\text {min }}=1$, o viés é monotonicamente decrescente com $H$, apesar da declividade de tal curva diminuir com o aumento de $H$. Adicionalmente, com o aumento de $N$, a dispersão das estimativas de $H$ tende a ser uma constante para os diversos valores de $H$. Para o caso em que $j_{\text {min }}=j_{\min }^{E Q M}$, o viés apresentou-se variável. Além disso, confirmou-se que a dispersão em tal caso é sempre maior do que para $j_{\text {min }}=1$, inclusive com tendência de aumentar em função de $H$ para os casos de modelos puramente LRD e para o caso de modelo com SRD tal que $f_{0} \approx 0$. 


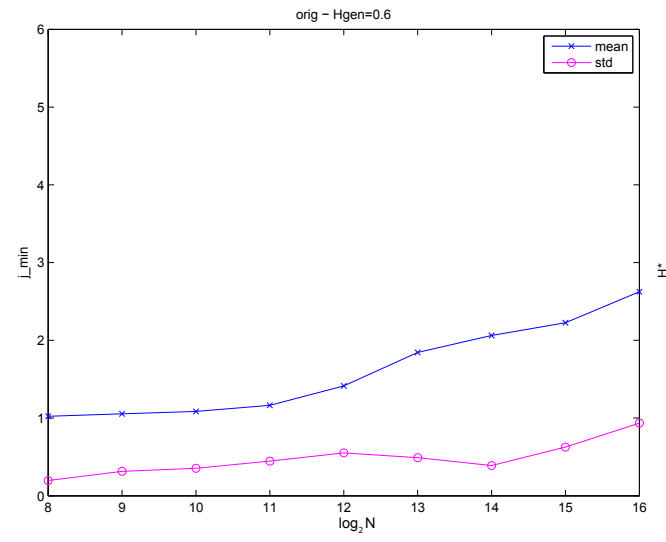

(a) Escala mínima EQM - $H=0,6$

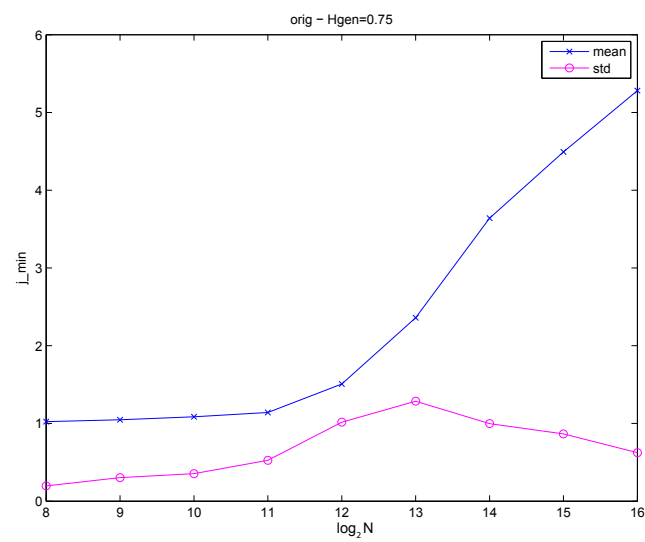

(c) Escala mínima $\mathrm{EQM}-H=0,75$

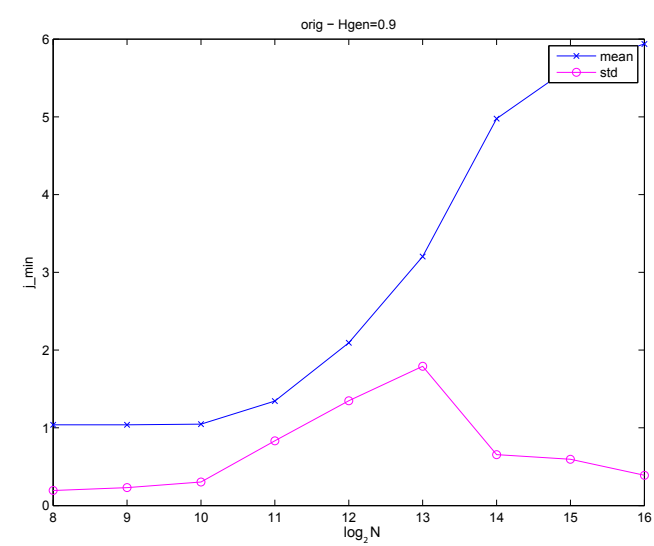

(e) Escala mínima EQM - $H=0,9$

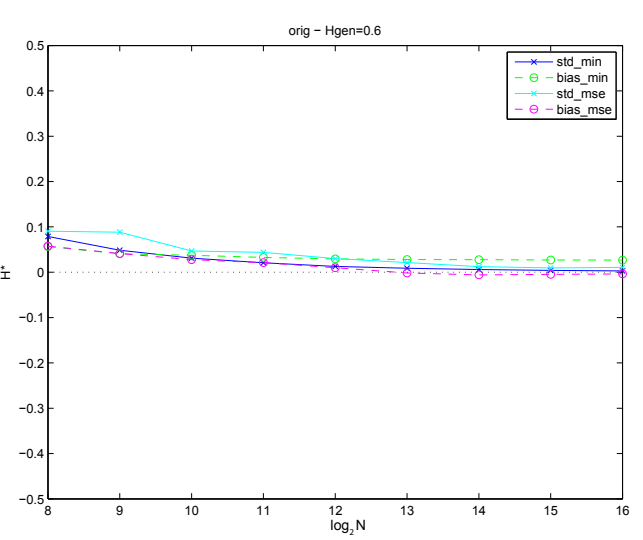

(b) Viés e variância de $\hat{H}$ para $j_{\text {min }}=1 \mathrm{e}$ $j_{\min }=j_{\min }^{E Q M}-H=0,6$

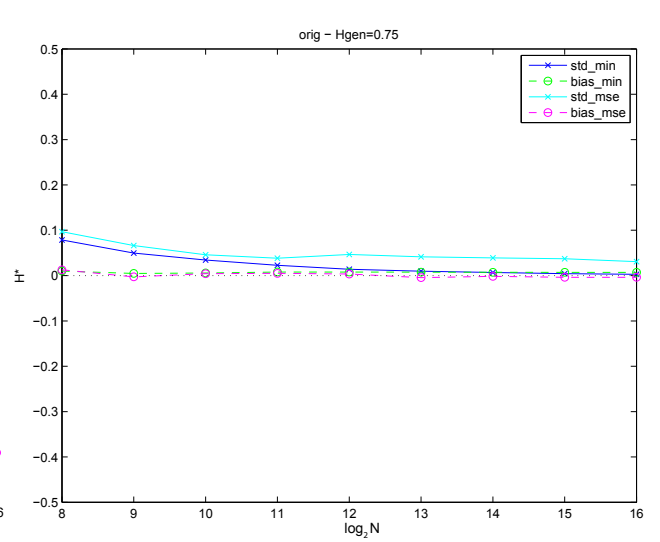

(d) Viés e variância de $\hat{H}$ para $j_{\text {min }}=1 \mathrm{e}$ $j_{\text {min }}=j_{\min }^{E Q M}-H=0,75$

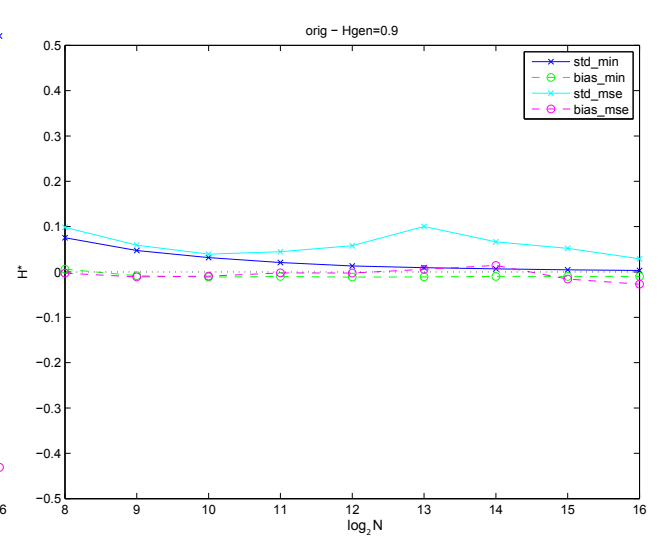

(f) Viés e variância de $\hat{H}$ para $j_{\text {min }}=1 \mathrm{e}$ $j_{\text {min }}=j_{\min }^{E Q M}-H=0,9$

Figura 6.45: Estudo da influência de $\log _{2} N$ na determinação de $j_{\min }^{E Q M}$ e de $\hat{H}$ - Séries originais sem SRD. 


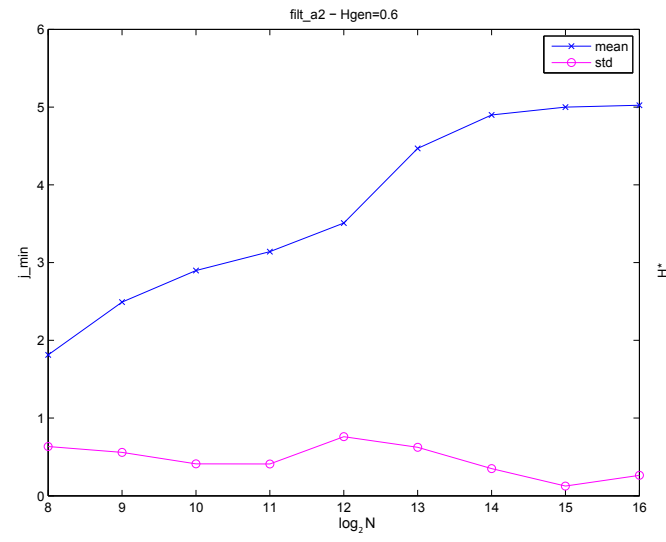

(a) Escala mínima EQM - $H=0,6$

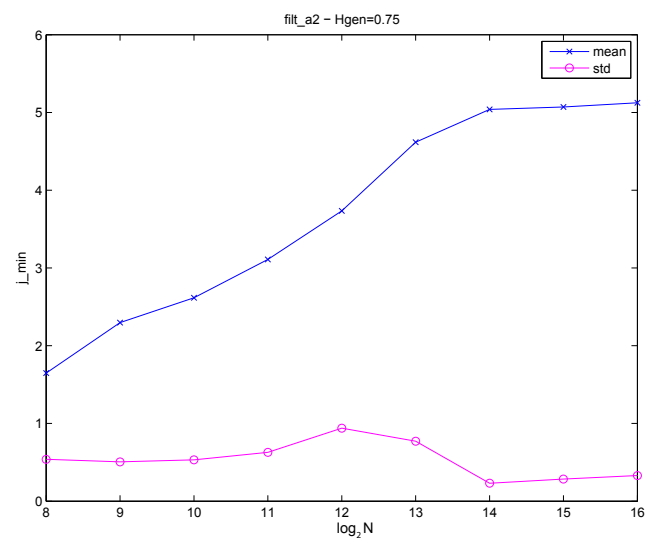

(c) Escala mínima EQM $-H=0,75$

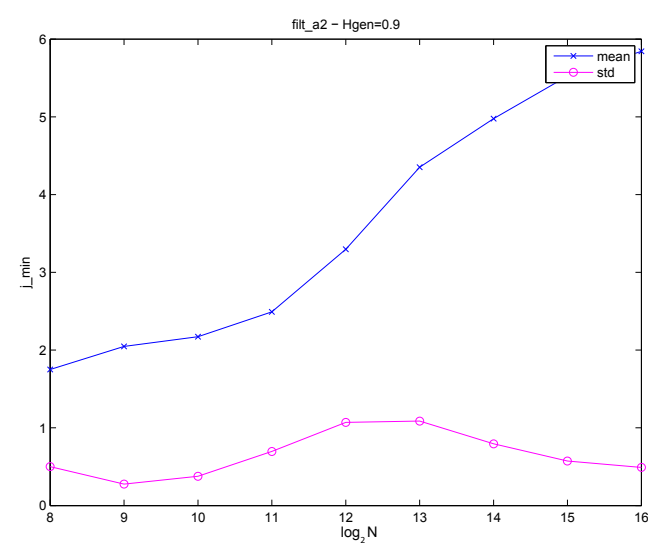

(e) Escala mínima EQM $-H=0,9$

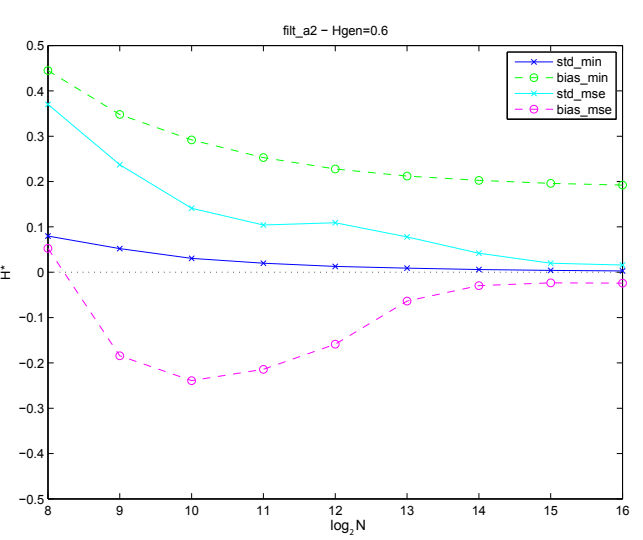

(b) Viés e variância de $\hat{H}$ para $j_{\text {min }}=1 \mathrm{e}$ $j_{\min }=j_{\min }^{E Q M}-H=0,6$

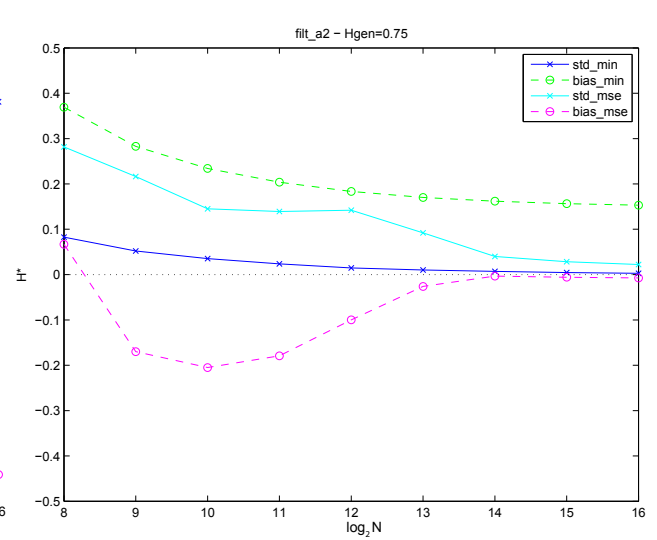

(d) Viés e variância de $\hat{H}$ para $j_{\text {min }}=1 \mathrm{e}$ $j_{\text {min }}=j_{\min }^{E Q M}-H=0,75$

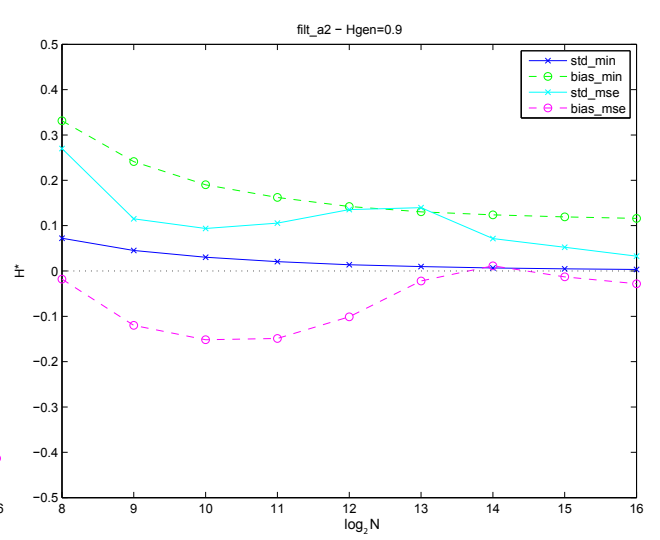

(f) Viés e variância de $\hat{H}$ para $j_{\text {min }}=1 \mathrm{e}$ $j_{\min }=j_{\min }^{E Q M}-H=0,9$

Figura 6.46: Estudo da influência de $\log _{2} N$ na determinação de $j_{\min }^{E Q M}$ e de $\hat{H}$ - Séries com SRD $-f_{0} \approx 0,2$. 


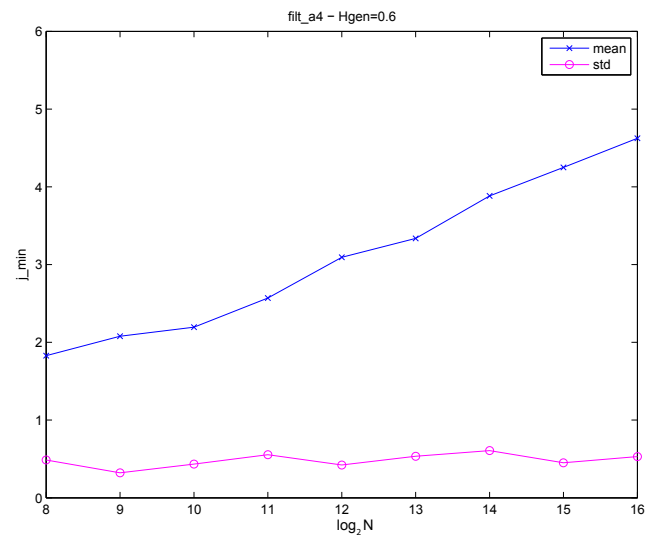

(a) Escala mínima EQM - $H=0,6$

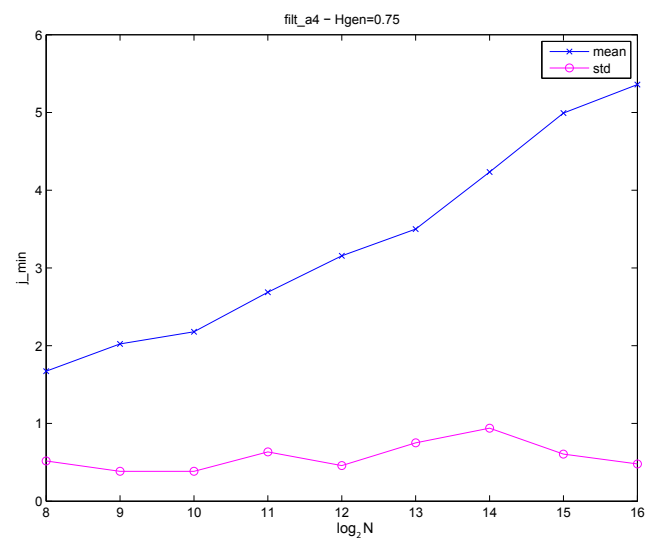

(c) Escala mínima $\mathrm{EQM}-H=0,75$

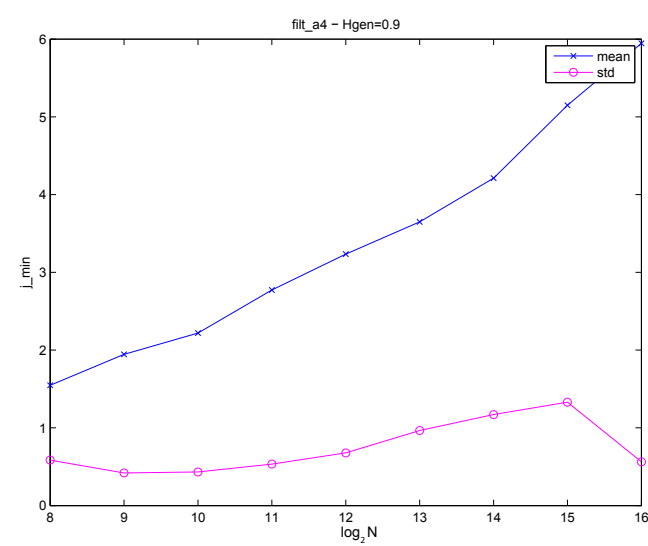

(e) Escala mínima EQM $-H=0,9$

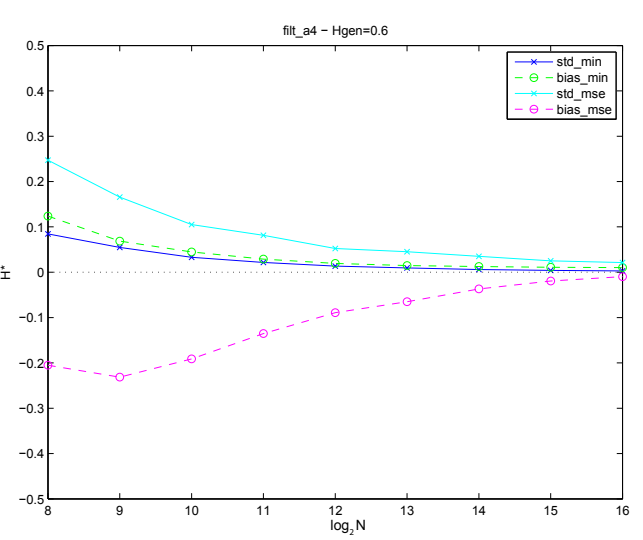

(b) Viés e variância de $\hat{H}$ para $j_{\text {min }}=1 \mathrm{e}$ $j_{\min }=j_{\min }^{E Q M}-H=0,6$

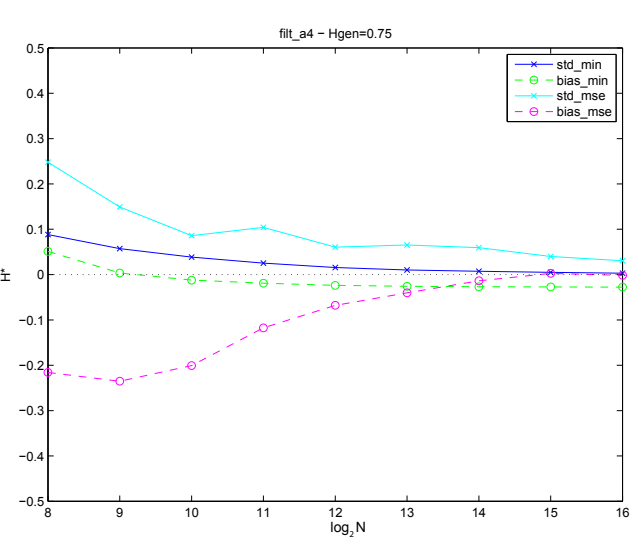

(d) Viés e variância de $\hat{H}$ para $j_{\min }=1 \mathrm{e}$ $j_{\text {min }}=j_{\min }^{E Q M}-H=0,75$

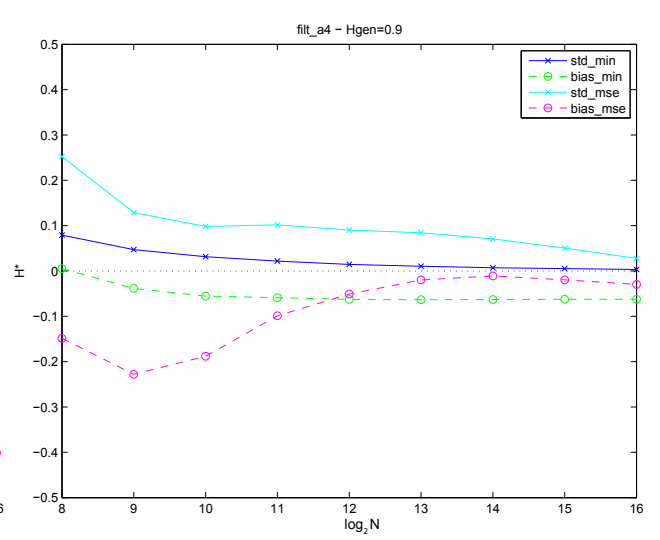

(f) Viés e variância de $\hat{H}$ para $j_{\text {min }}=1 \mathrm{e}$ $j_{\min }=j_{\min }^{E Q M}-H=0,9$

Figura 6.47: Estudo da influência de $\log _{2} N$ na determinação de $j_{\min }^{E Q M}$ e de $\hat{H}$ - Séries com SRD $-f_{0} \approx 0,4$. 


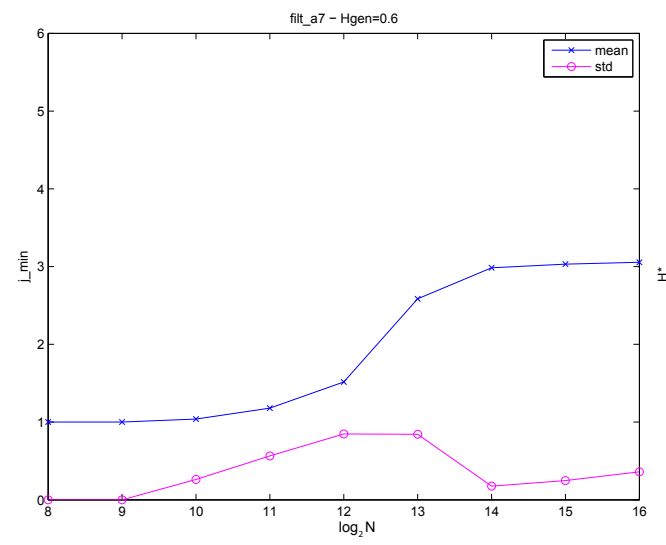

(a) Escala mínima EQM - $H=0,6$

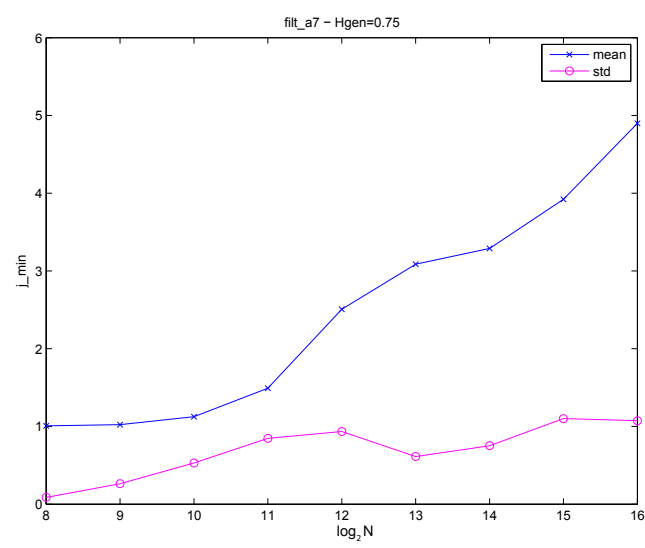

(c) Escala mínima $\mathrm{EQM}-H=0,75$

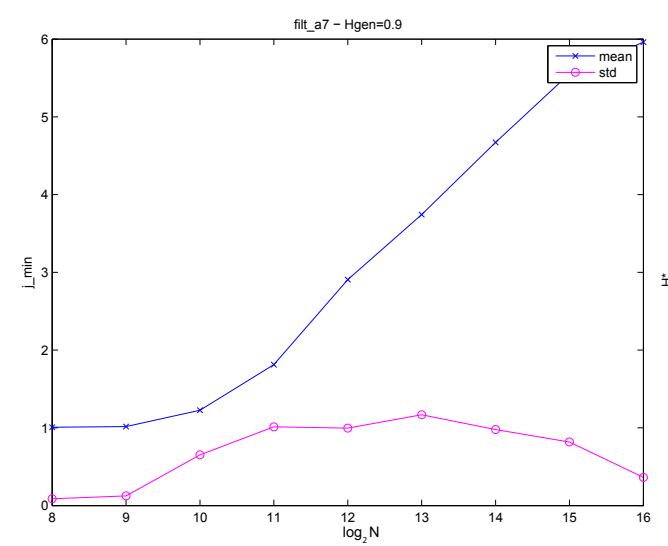

(e) Escala mínima EQM $-H=0,9$

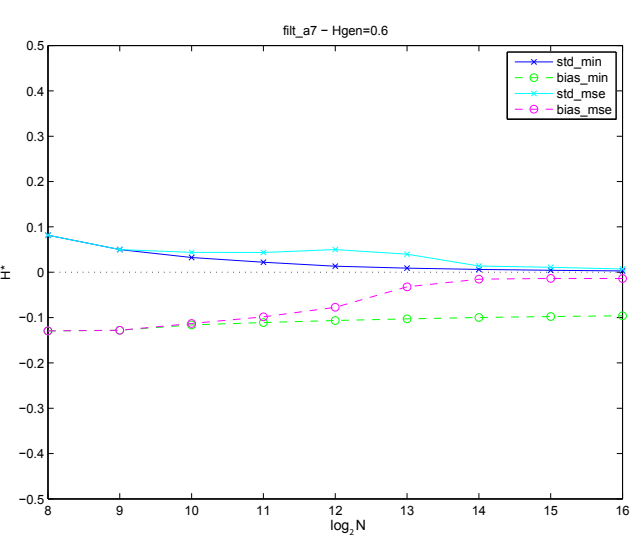

(b) Viés e variância de $\hat{H}$ para $j_{\min }=1 \mathrm{e}$ $j_{\min }=j_{\min }^{E Q M}-H=0,6$

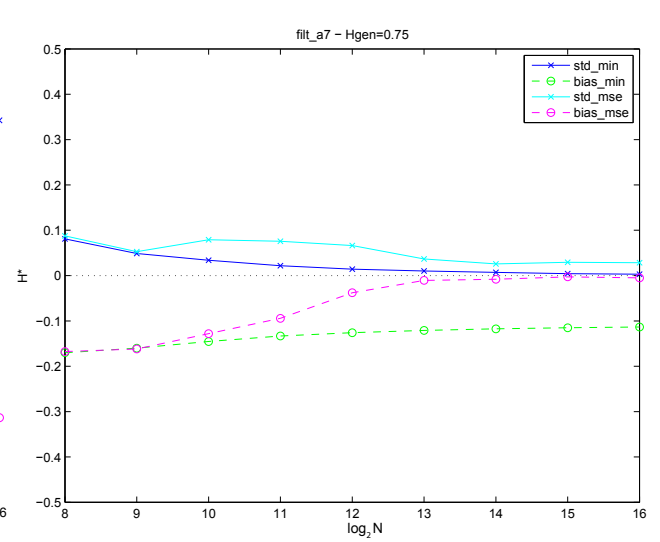

(d) Viés e variância de $\hat{H}$ para $j_{\text {min }}=1 \mathrm{e}$ $j_{\text {min }}=j_{\min }^{E Q M}-H=0,75$

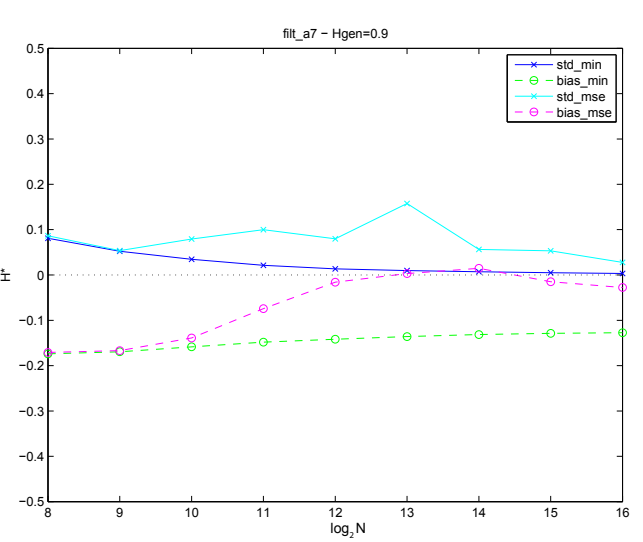

(f) Viés e variância de $\hat{H}$ para $j_{\text {min }}=1 \mathrm{e}$ $j_{\min }=j_{\min }^{E Q M}-H=0,9$

Figura 6.48: Estudo da influência de $\log _{2} N$ na determinação de $j_{\min }^{E Q M}$ e de $\hat{H}$ - Séries com SRD - $f_{0} \approx 0,7$. 


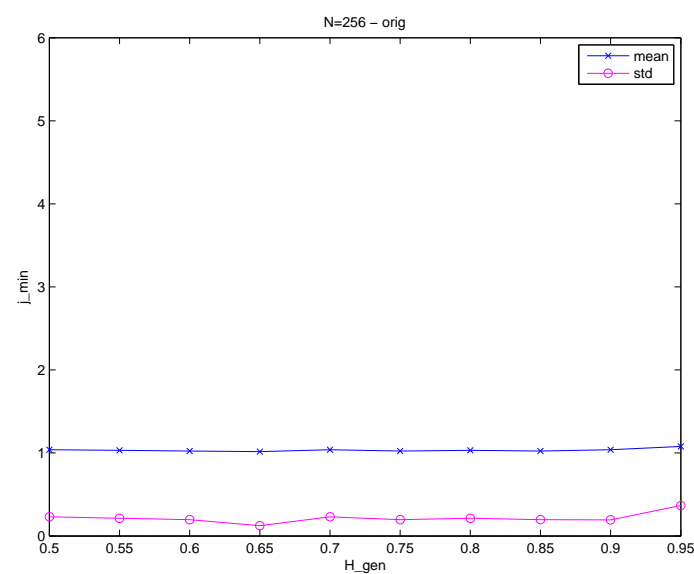

(a) Escala mínima EQM - Séries originais sem SRD

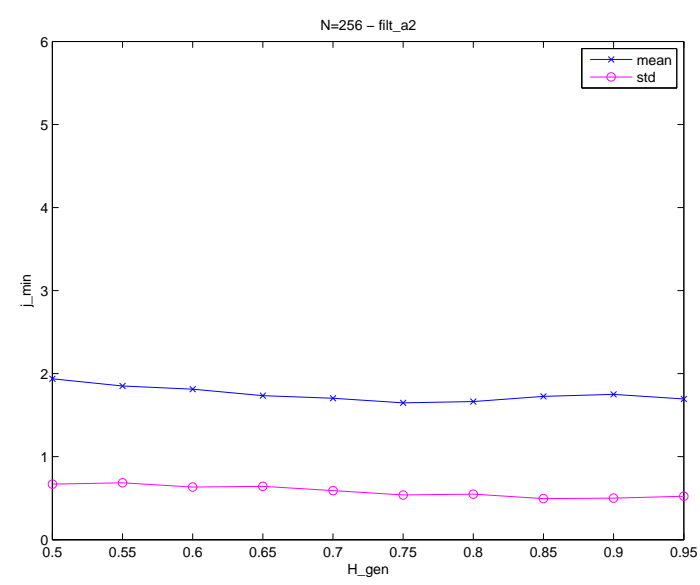

(c) Escala mínima EQM - Séries com SRD $f_{0} \approx 0,2$

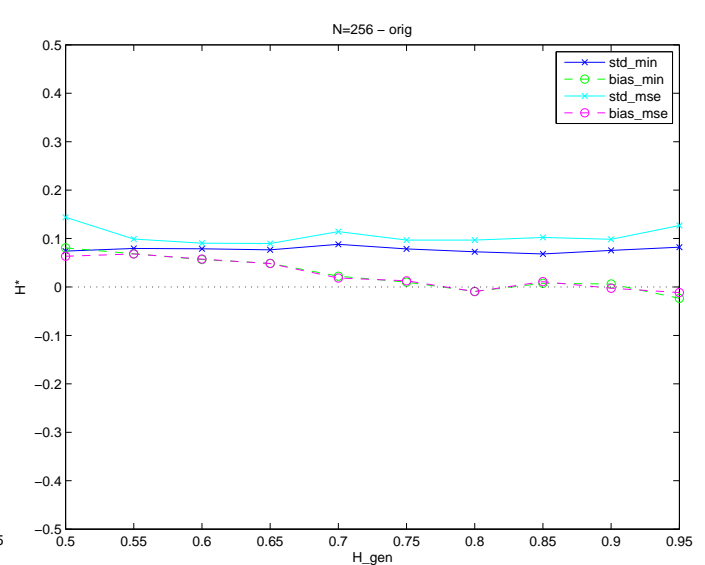

(b) Viés e variância de $\hat{H}$ para $j_{\min }=1 \mathrm{e}$ $j_{\min }=j_{\min }^{E Q M}-$ Séries originais sem SRD

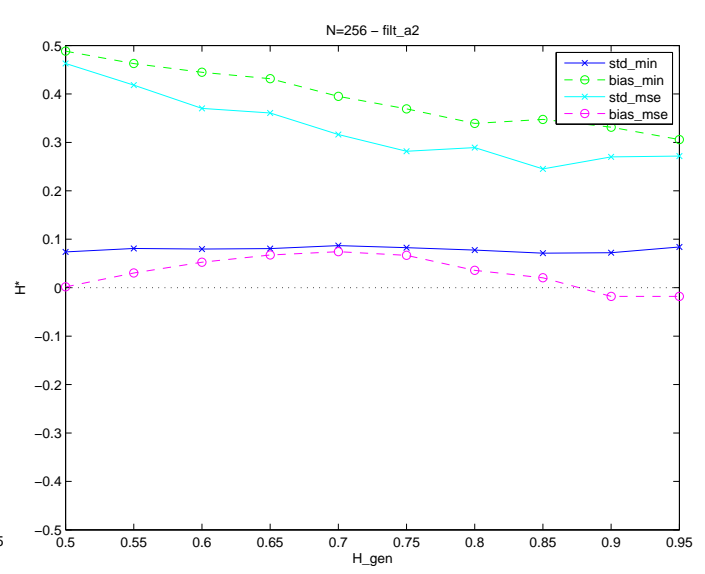

(d) Viés e variância de $\hat{H}$ para $j_{\text {min }}=1 \mathrm{e}$ $j_{\min }=j_{\min }^{E Q M}-$ Séries com SRD $-f_{0} \approx 0,2$ $j_{\min }=j_{\min }^{E Q M}-$ Séries com SRD $-f_{0} \approx 0,2$
$H_{g e n}$ na determinação de $j_{m i n}^{E Q M}$ e de $\hat{H}$

Figura 6.49: Estudo da influência de $H_{g e n}$ na determinação de $j_{\min }^{E Q M}$ e de $\hat{H}-$ Séries com $N=2^{8}$ (parte 1$)$. 


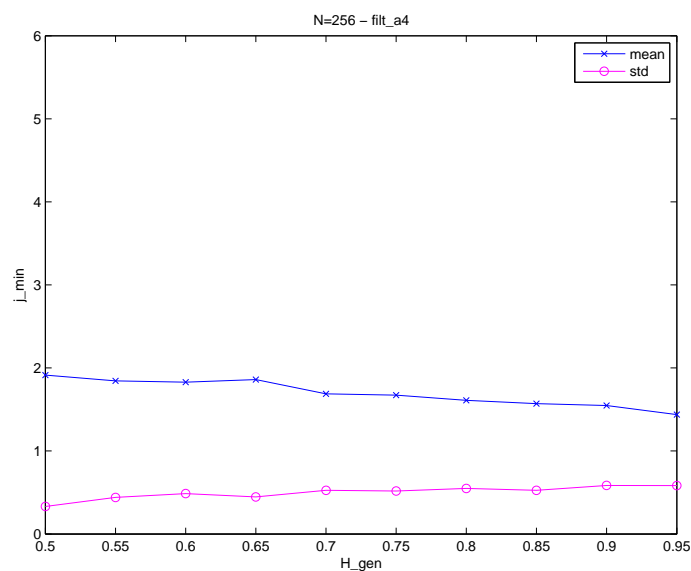

(a) Escala mínima EQM - Séries com SRD $f_{0} \approx 0,4$

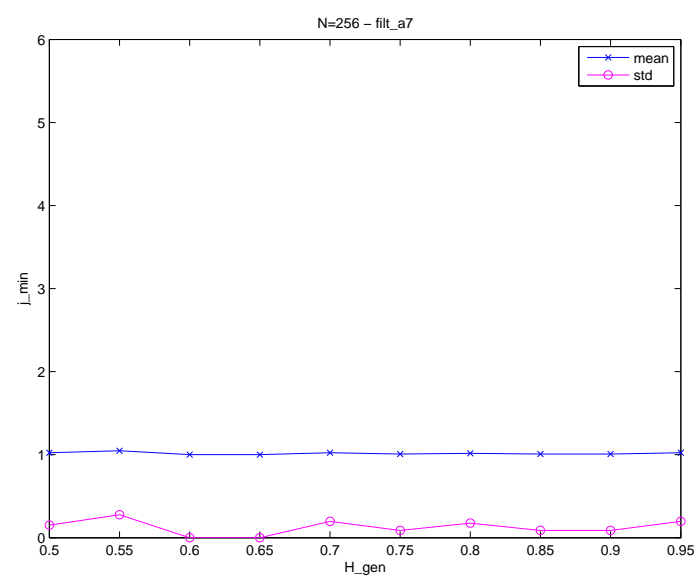

(c) Escala mínima EQM - Séries com SRD $f_{0} \approx 0,7$

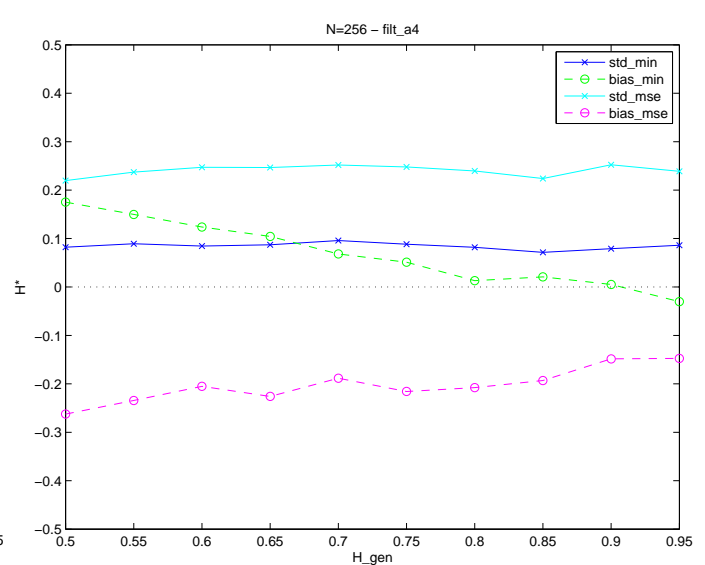

(b) Viés e variância de $\hat{H}$ para $j_{\text {min }}=1 \mathrm{e}$ $j_{\text {min }}=j_{\text {min }}^{E Q M}-$ Séries com SRD $-f_{0} \approx 0,4$

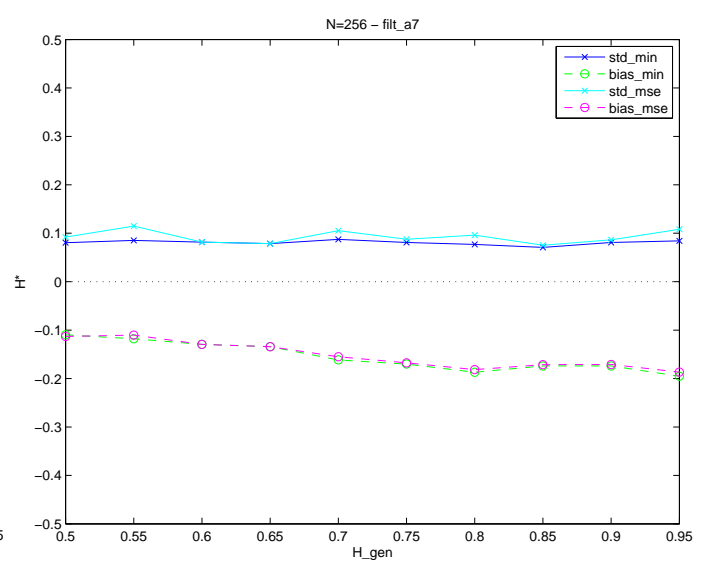

(d) Viés e variância de $\hat{H}$ para $j_{\text {min }}=1 \mathrm{e}$ $j_{\text {min }}=j_{\text {min }}^{E Q M}-$ Séries com SRD $-f_{0} \approx 0,7$

Figura 6.50: Estudo da influência de $H_{\text {gen }}$ na determinação de $j_{\min }^{E Q M}$ e de $\hat{H}-$ Séries com $N=2^{8}$ (parte 2). 


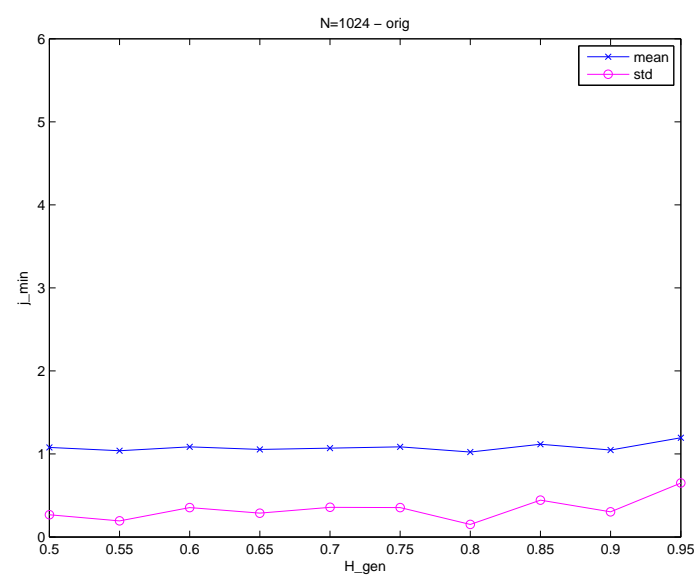

(a) Escala mínima EQM - Séries originais sem SRD

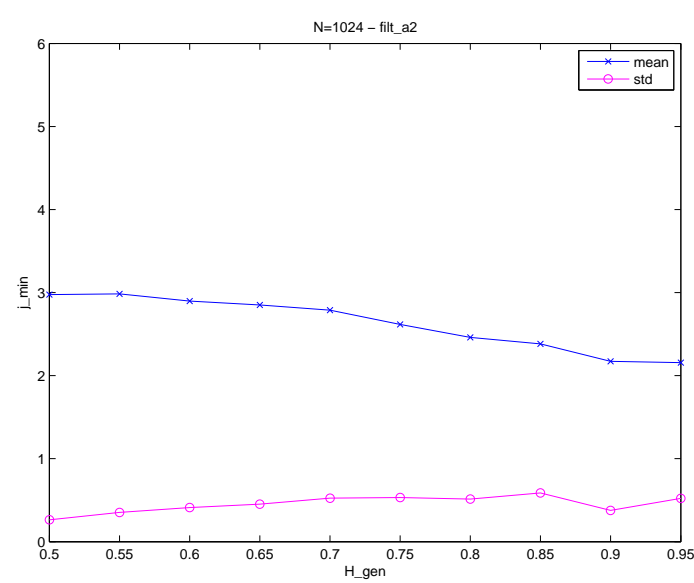

(c) Escala mínima EQM - Séries com SRD $f_{0} \approx 0,2$

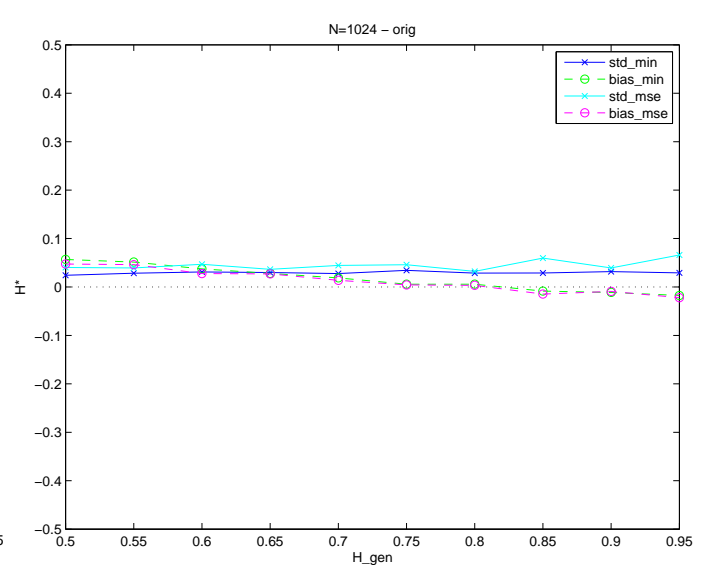

(b) Viés e variância de $\hat{H}$ para $j_{\text {min }}=1$ e $j_{\text {min }}=j_{\min }^{E Q M}-$ Séries originais sem SRD

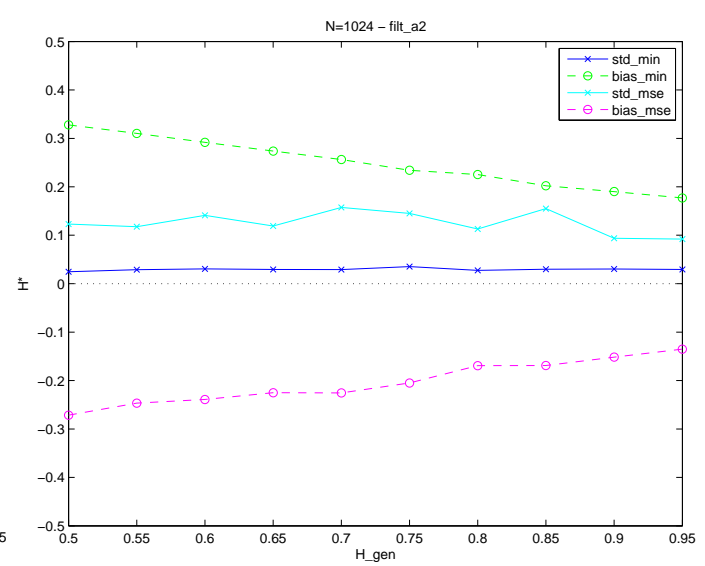

(d) Viés e variância de $\hat{H}$ para $j_{\text {min }}=1 \mathrm{e}$ $j_{\text {min }}=j_{\min }^{E Q M}-$ Séries com SRD $-f_{0} \approx 0,2$

$j_{\min }=j_{\min }^{E Q M}-$ Séries com SRD $-f_{0} \approx 0,2$
Figura 6.51: Estudo da influência de $H_{\text {gen }}$ na determinação de $j_{\min }^{E Q M}$ e de $\hat{H}-$ Séries com $N=2^{10}$ (parte 1 ). 


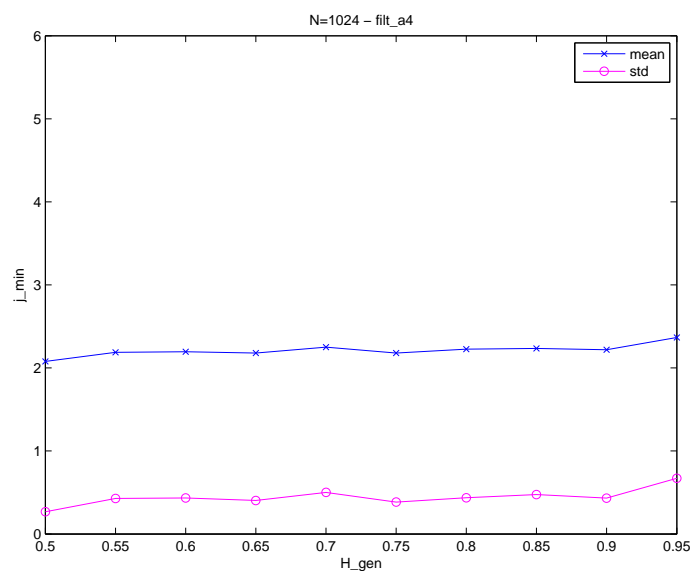
$f_{0} \approx 0,4$

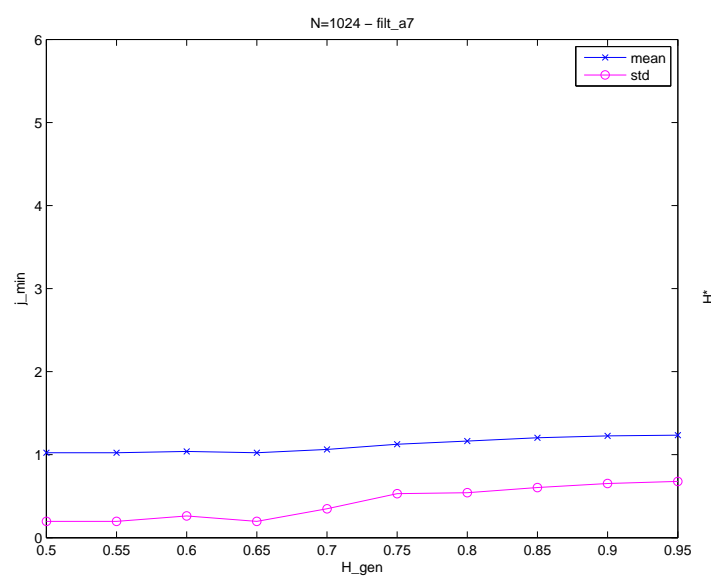

(c) Escala mínima EQM - Séries com SRD $f_{0} \approx 0,7$ (a) Escala mínima EQM - Séries com SRD -

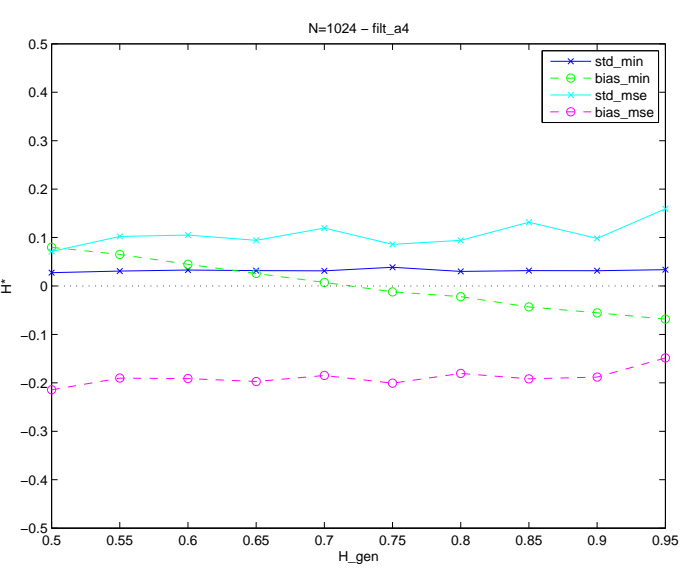

(b) Viés e variância de $\hat{H}$ para $j_{\text {min }}=1 \mathrm{e}$ $j_{\text {min }}=j_{\text {min }}^{E Q M}-$ Séries com SRD $-f_{0} \approx 0,4$

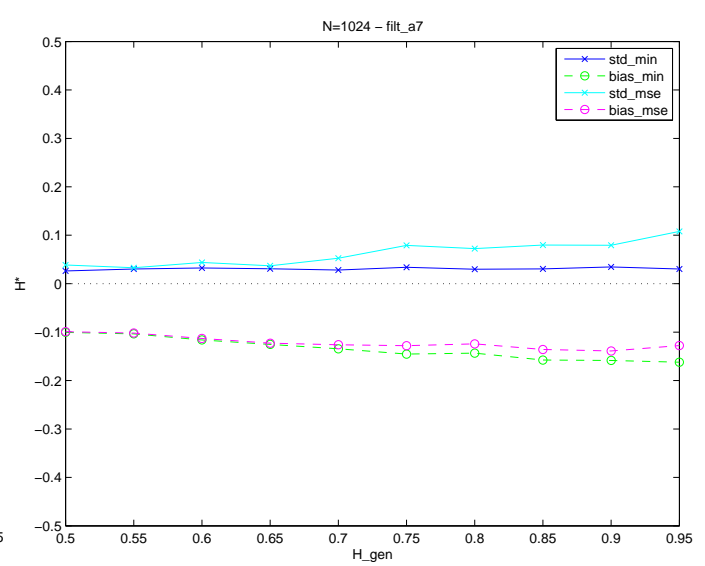

(d) Viés e variância de $\hat{H}$ para $j_{\text {min }}=1 \mathrm{e}$ $j_{\text {min }}=j_{\text {min }}^{E Q M}-$ Séries com SRD $-f_{0} \approx 0,7$ $j_{\text {min }}=j_{\text {min }}$ - Séries com SRD $-f_{0} \approx 0,7$

Figura 6.52: Estudo da influência de $H_{\text {gen }}$ na determinação de $j_{\min }^{E Q M}$ e de $\hat{H}-$ Séries com $N=2^{10}$ (parte 2). 


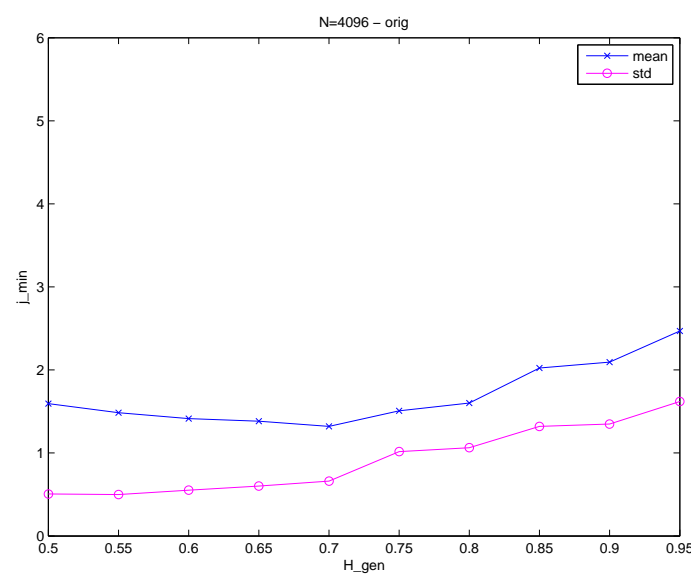

(a) Escala mínima EQM - Séries originais sem SRD

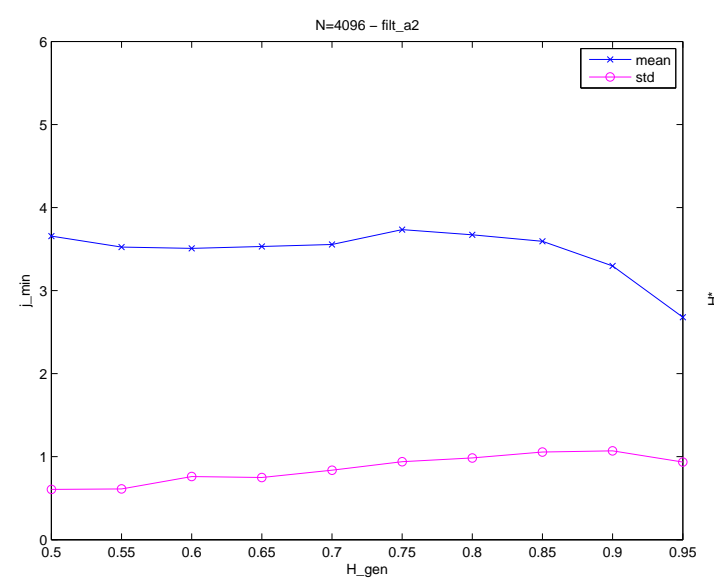

(c) Escala mínima EQM - Séries com SRD $f_{0} \approx 0,2$

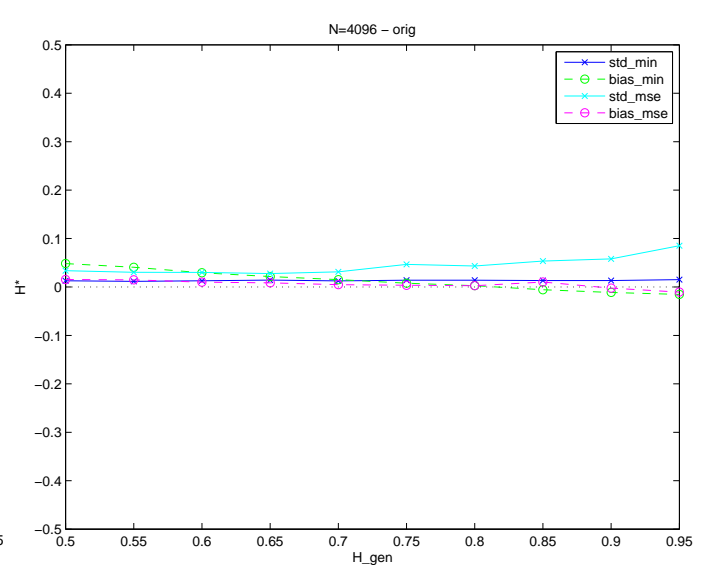

(b) Viés e variância de $\hat{H}$ para $j_{\text {min }}=1$ e $j_{\text {min }}=j_{\min }^{E Q M}-$ Séries originais sem SRD

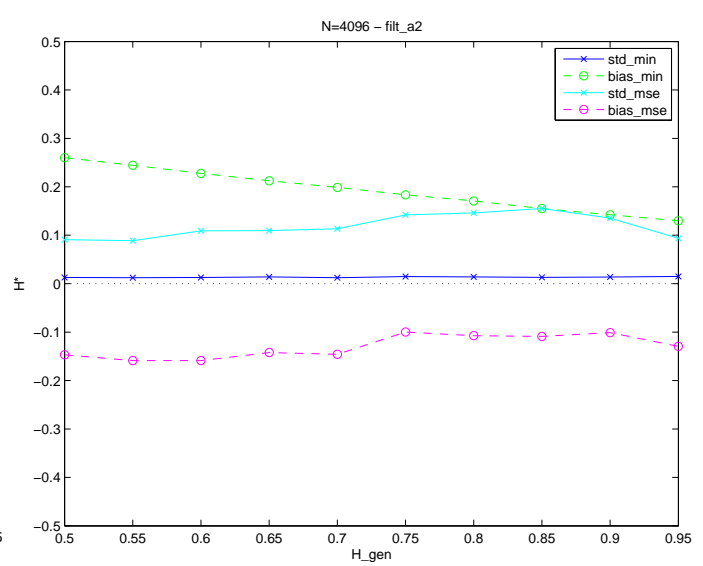

(d) Viés e variância de $\hat{H}$ para $j_{\text {min }}=1 \mathrm{e}$ $j_{\text {min }}=j_{\min }^{E Q M}-$ Séries com SRD $-f_{0} \approx 0,2$

$j_{\min }=j_{\min }^{E Q M}-$ Séries com SRD $-f_{0} \approx 0,2$
Figura 6.53: Estudo da influência de $H_{\text {gen }}$ na determinação de $j_{\min }^{E Q M}$ e de $\hat{H}-$ Séries com $N=2^{12}$ (parte 1 ). 


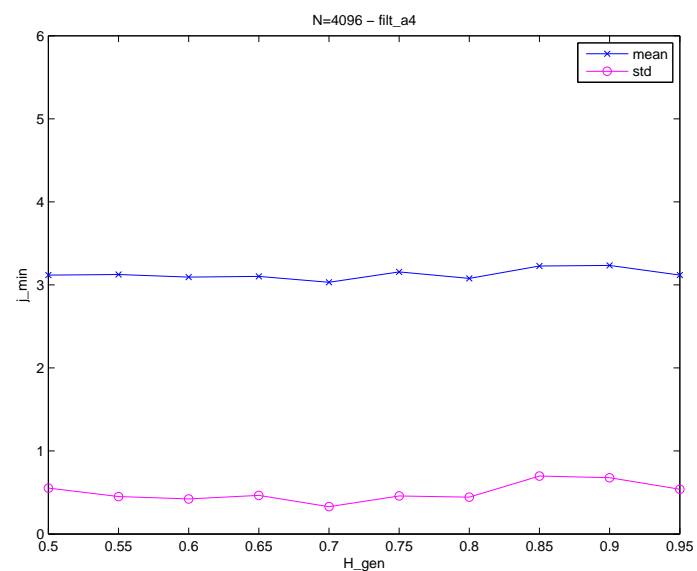

(a) Escala mínima EQM - Séries com SRD $f_{0} \approx 0,4$

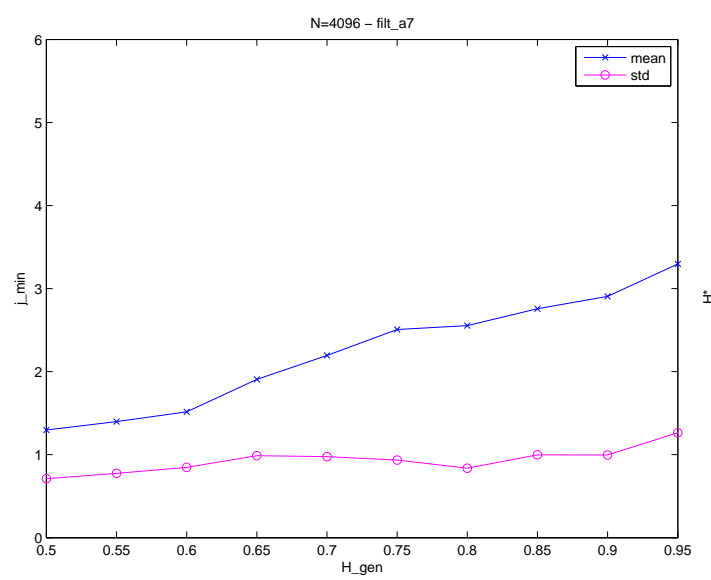

(c) Escala mínima EQM - Séries com SRD $f_{0} \approx 0,7$

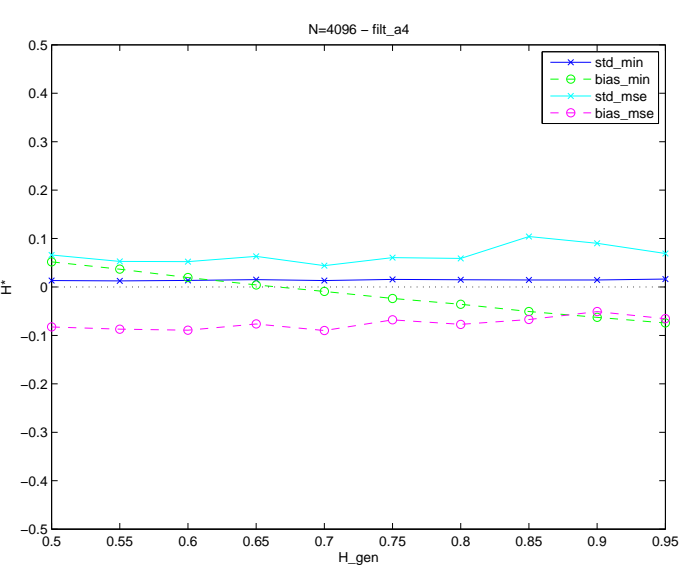

(b) Viés e variância de $\hat{H}$ para $j_{\text {min }}=1 \mathrm{e}$ $j_{\text {min }}=j_{\text {min }}^{E Q M}-$ Séries com SRD $-f_{0} \approx 0,4$

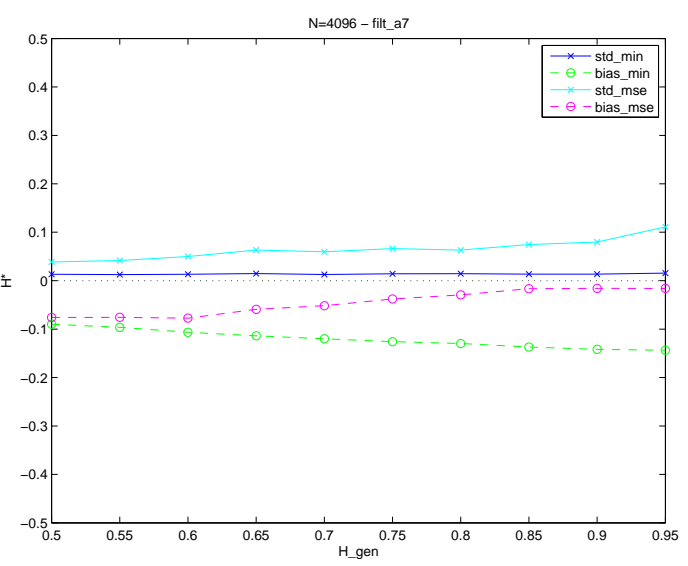

(d) Viés e variância de $\hat{H}$ para $j_{\text {min }}=1 \mathrm{e}$ $j_{\text {min }}=j_{\text {min }}^{E Q M}-$ Séries com SRD $-f_{0} \approx 0,7$ $j_{\text {min }}=j_{\text {min }}-$ Séries com SRD $-f_{0} \approx 0,7$

Figura 6.54: Estudo da influência de $H_{\text {gen }}$ na determinação de $j_{\min }^{E Q M}$ e de $\hat{H}-$ Séries com $N=2^{12}$ (parte 2). 


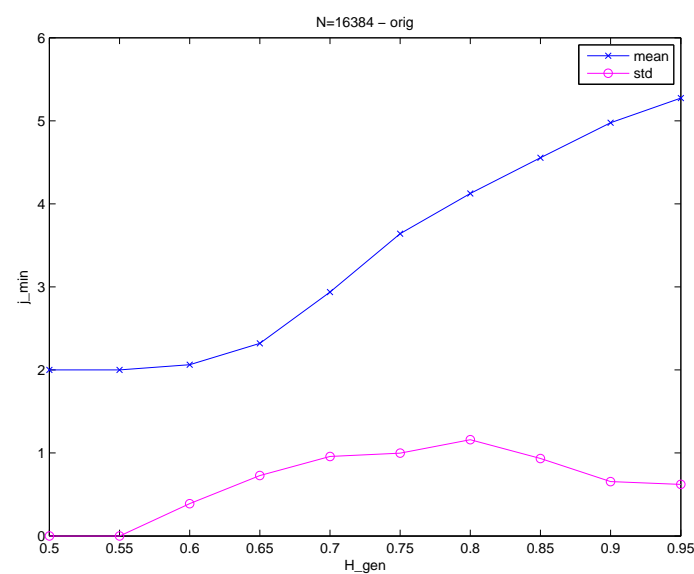

(a) Escala mínima EQM - Séries originais sem SRD

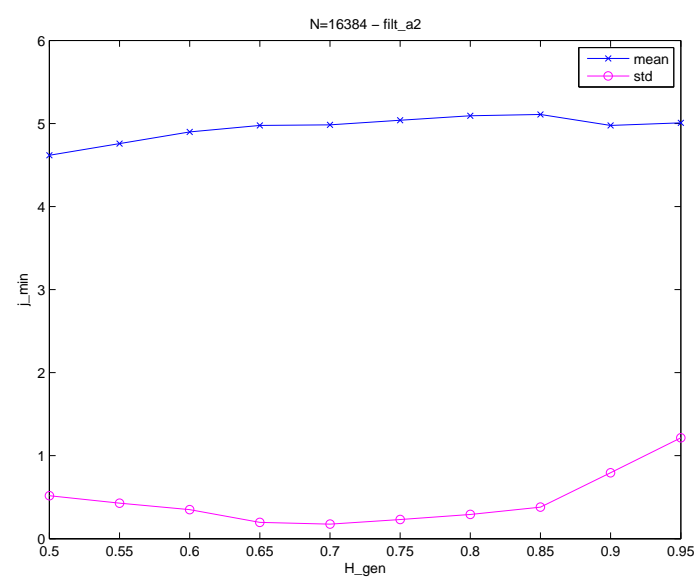

(c) Escala mínima EQM - Séries com SRD $f_{0} \approx 0,2$

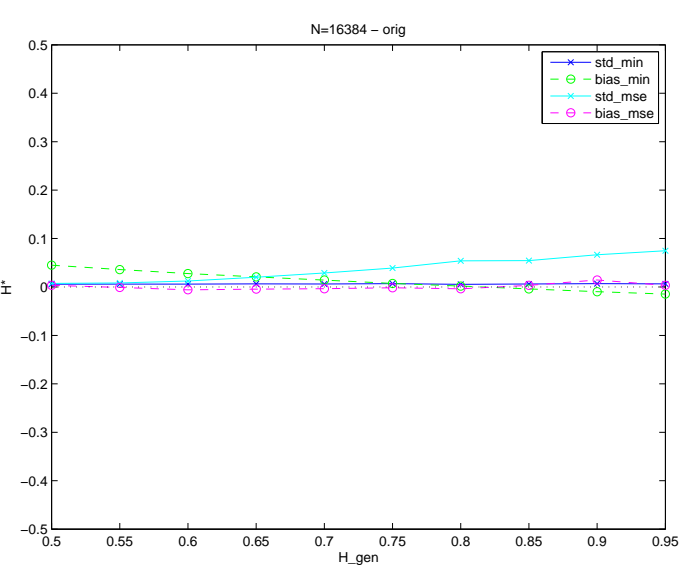

(b) Viés e variância de $\hat{H}$ para $j_{\min }=1 \mathrm{e}$ $j_{\min }=j_{\min }^{E Q M}-$ Séries originais sem SRD

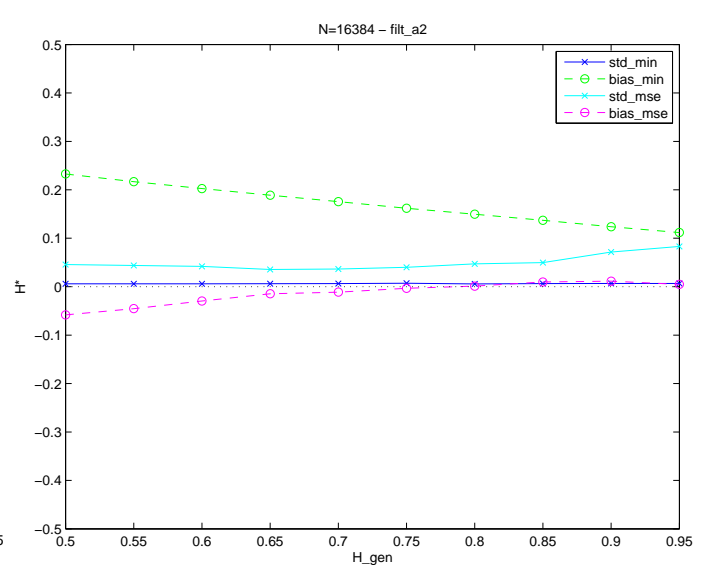

(d) Viés e variância de $\hat{H}$ para $j_{\text {min }}=1 \mathrm{e}$ $j_{\text {min }}=j_{\min }^{E Q M}-$ Séries com SRD $-f_{0} \approx 0,2$

Figura 6.55: Estudo da influência de $H_{g e n}$ na determinação de $j_{\min }^{E Q M}$ e de $\hat{H}-$ Séries com $N=2^{14}$ (parte 1 ). 


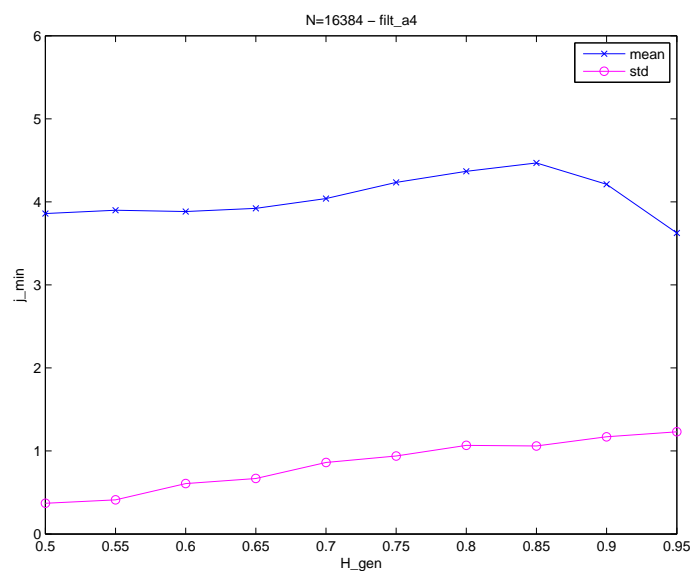

(a) Escala mínima EQM - Séries com SRD $f_{0} \approx 0,4$

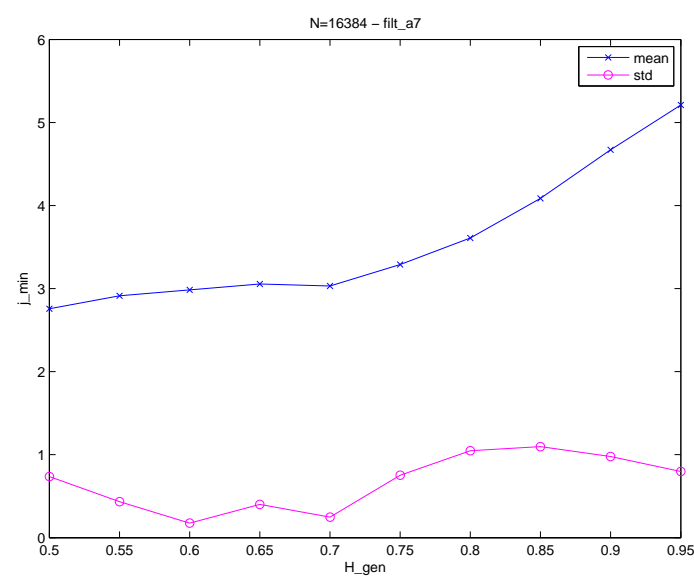

(c) Escala mínima EQM - Séries com SRD $f_{0} \approx 0,7$

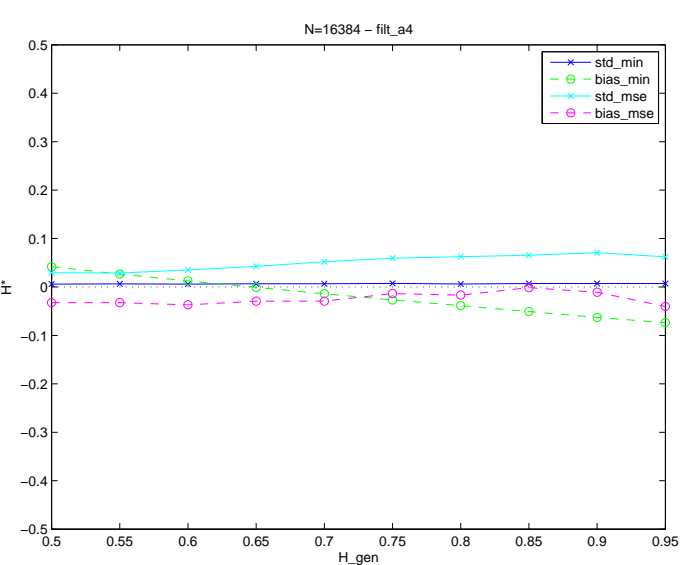

(b) Viés e variância de $\hat{H}$ para $j_{\text {min }}=1 \mathrm{e}$ $j_{\text {min }}=j_{\text {min }}^{E Q M}-$ Séries com SRD $-f_{0} \approx 0,4$

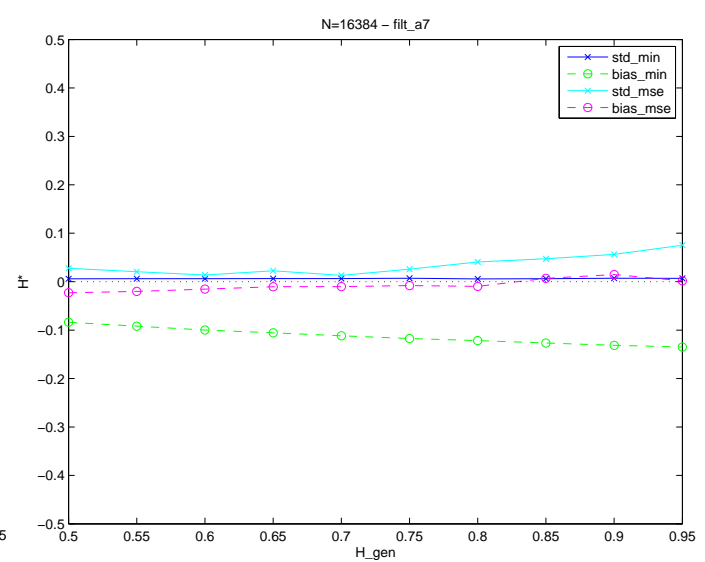

(d) Viés e variância de $\hat{H}$ para $j_{\min }=1 \mathrm{e}$ $j_{\text {min }}=j_{\text {min }}^{E Q M}-$ Séries com SRD $-f_{0} \approx 0,7$ $j_{\min }=j_{\min }-$ Séries com SRD $-f_{0} \approx 0,7$

Figura 6.56: Estudo da influência de $H_{\text {gen }}$ na determinação de $j_{\min }^{E Q M}$ e de $\hat{H}$ Séries com $N=2^{14}$ (parte 2). 


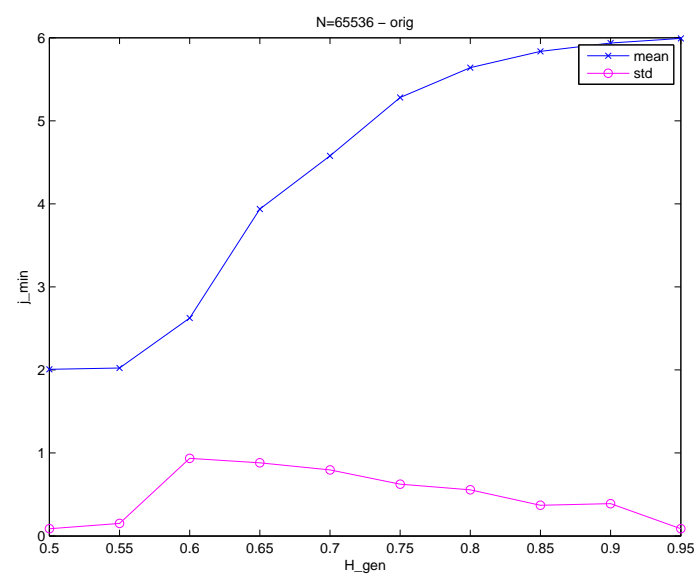

(a) Escala mínima EQM - Séries originais sem SRD

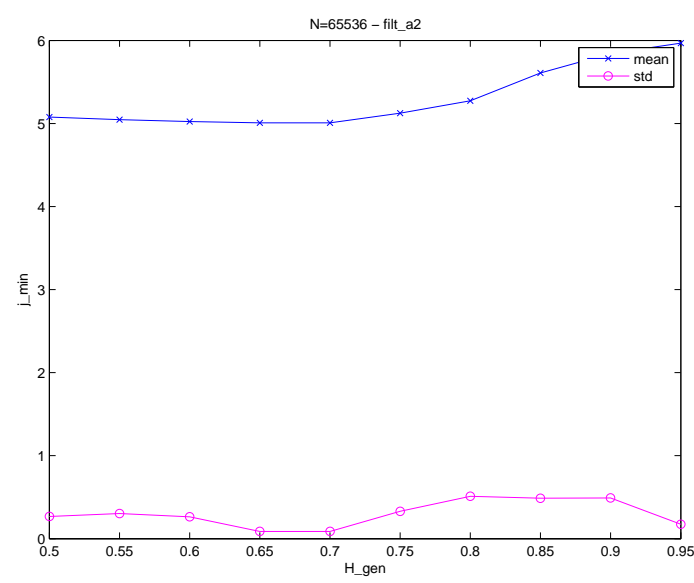

(c) Escala mínima EQM - Séries com SRD $f_{0} \approx 0,2$

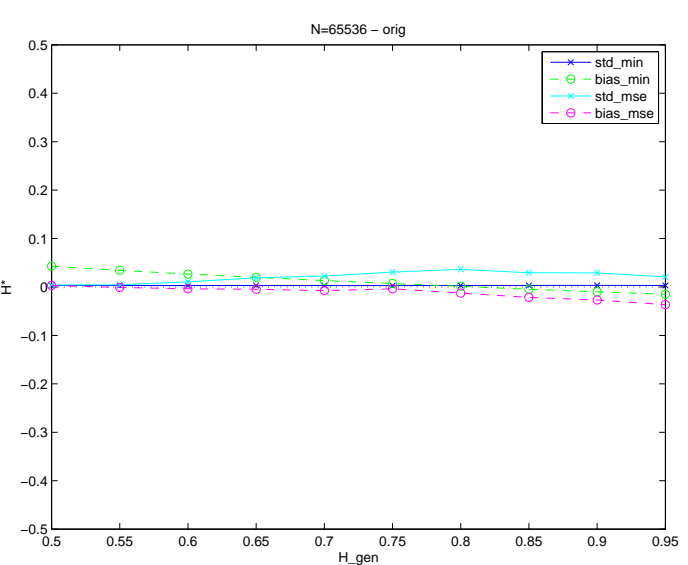

(b) Viés e variância de $\hat{H}$ para $j_{\text {min }}=1$ e $j_{\text {min }}=j_{\min }^{E Q M}-$ Séries originais sem SRD

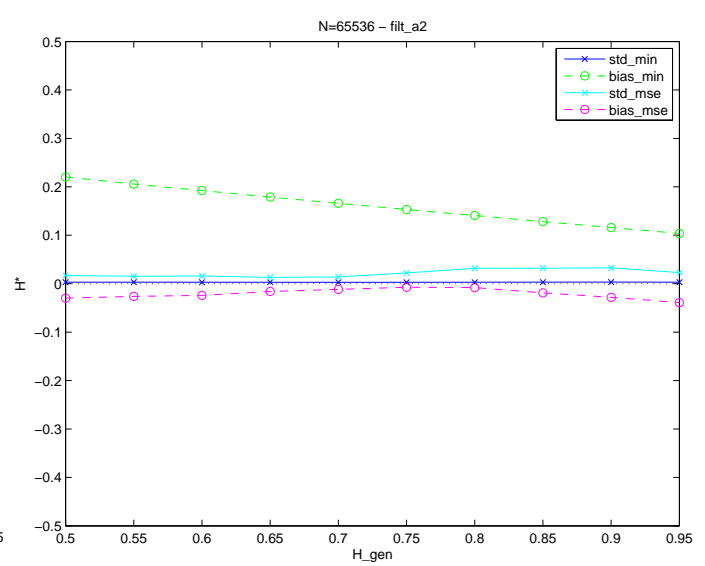

(d) Viés e variância de $\hat{H}$ para $j_{\text {min }}=1 \mathrm{e}$ $j_{\text {min }}=j_{\text {min }}^{E Q M}-$ Séries com SRD $-f_{0} \approx 0,2$

Figura 6.57: Estudo da influência de $H_{g e n}$ na determinação de $j_{\min }^{E Q M}$ e de $\hat{H}$ Séries com $N=2^{16}$ (parte 1$)$. 


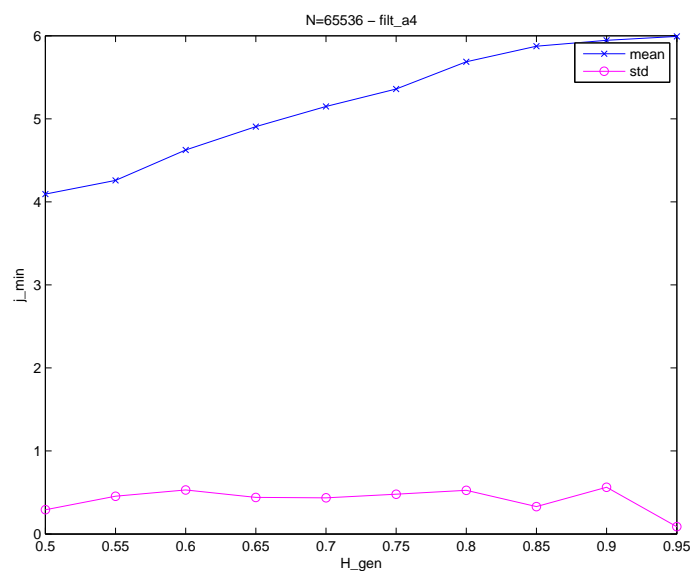

(a) Escala mínima EQM - Séries com SRD $f_{0} \approx 0,4$

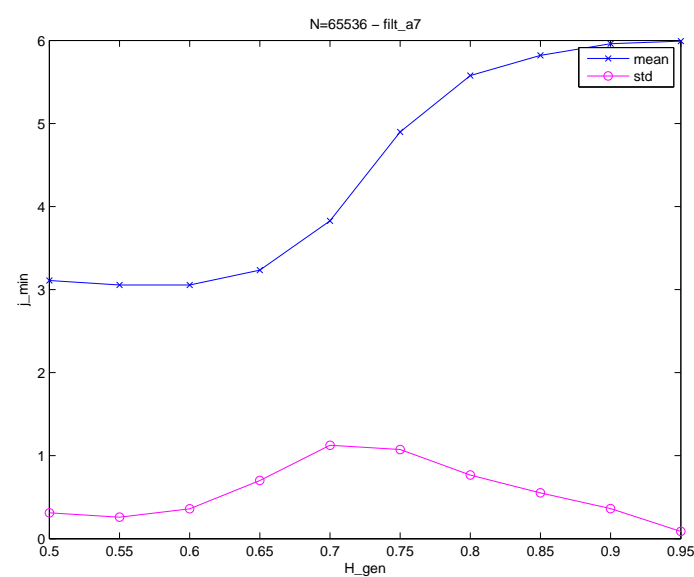

(c) Escala mínima EQM - Séries com SRD $f_{0} \approx 0,7$

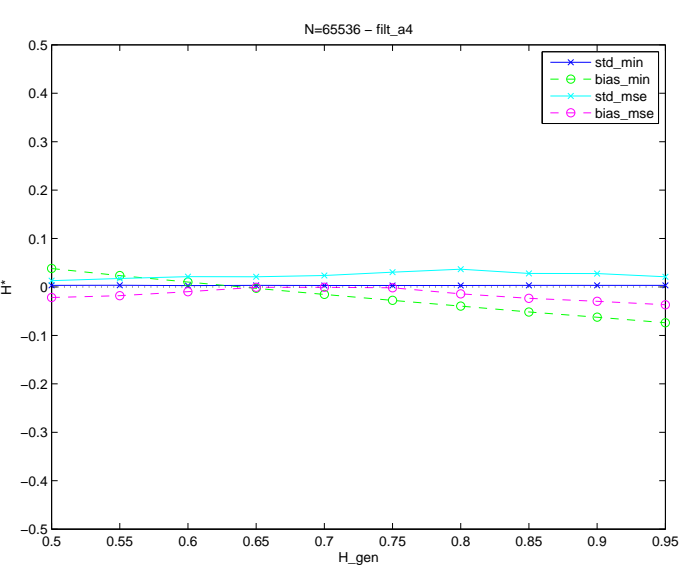

(b) Viés e variância de $\hat{H}$ para $j_{\text {min }}=1 \mathrm{e}$ $j_{\text {min }}=j_{\text {min }}^{E Q M}-$ Séries com SRD $-f_{0} \approx 0,4$

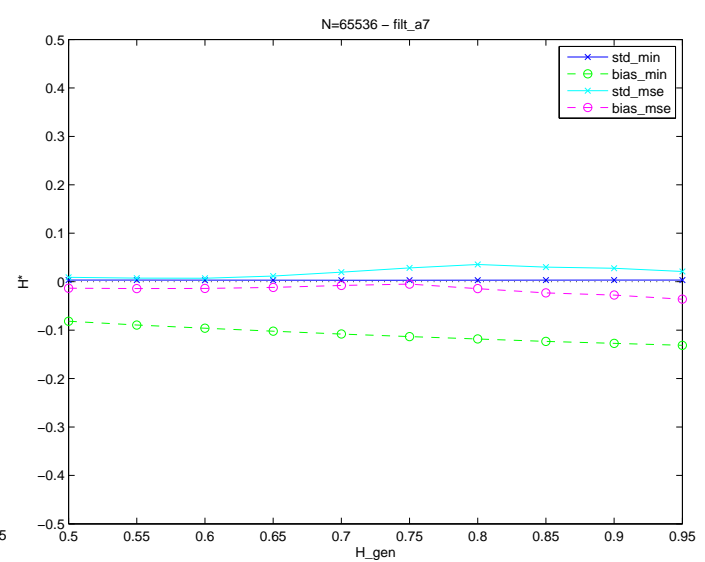

(d) Viés e variância de $\hat{H}$ para $j_{\min }=1 \mathrm{e}$ $j_{\text {min }}=j_{\text {min }}^{E Q M}-$ Séries com SRD $-f_{0} \approx 0,7$

Figura 6.58: Estudo da influência de $H_{\text {gen }}$ na determinação de $j_{\min }^{E Q M}$ e de $\hat{H}-$ Séries com $N=2^{16}$ (parte 2). 


\subsubsection{Síntese dos resultados}

A Tabela 6.6 apresenta uma síntese dos principais resultados expostos na Seção 6.4 .

Tabela 6.6: Resultados da Seção 6.4.

\begin{tabular}{|c|l|}
\hline \hline Resultado & Descrição \\
\hline \hline 1 & $\begin{array}{l}\text { Apesar da coerência entre os resultados de [10] e do presente trabalho, não foi } \\
\text { possível comparar diretamente os resultados de ambos os trabalhos uma vez } \\
\text { que utilizaram-se de modelos distintos em seus estudos }\end{array}$ \\
\hline 2 & $\begin{array}{l}\text { As tabelas e gráficos com os resultados detalhados do comportamento das esti- } \\
\text { mativas de } H \text { nos diversos casos apresentados não são de forma alguma exaus- } \\
\text { tivos, cabendo estudos futuros dos mesmos }\end{array}$ \\
\hline 3 & $\begin{array}{l}\text { As tabelas e gráficos possibilitam que propostas de melhoria do método de } \\
\text { estimação possam ser realizadas, como a proposta de estimação utilizando-se } \\
j_{m i n}=1 \text { (devido à baixa dispersão das estimativas) com a posterior utilização } \\
\text { dos dados deste trabalho para correção do viés apresentado. }\end{array}$ \\
\hline
\end{tabular}




\section{$7 \quad$ DWPT}

Conforme anteriormente descrito, o método de Abry-Veitch baseia-se, fundamentalmente, na utilização do espectro wavelet para determinação de uma região de tal espectro em que possa ser identificado o fenômeno de scaling, do qual o LRD é uma das manifestações de tal fenômeno. Entretanto, o espectro wavelet utilizado baseia-se na DWT que, conforme também anteriormente descrito, não produz um resultado tão "rico" quanto a DWPT, uma vez que esta última possibilita uma maior flexibilidade na resolução tempo-frequência. A presente Seção explorará a utilização do espectro wavelet baseado na DWPT para a estimação do parâmetro $H$, assim como comparar tal estimação com a obtida utilizando-se o espectro baseado na DWT.

\subsection{Condições experimentais}

As séries utilizadas na presente Seção seguem as mesmas condições de geração descritas na Seção 6.4.1, com a exceção de que, para cada caso descrito em tal Seção, foi utilizada apenas a primeira realização para efeitos de simplicidade da análise.

Desta maneira, tais séries foram decompostas pela DWPT utilizando os filtros de Daubechies db1 (também conhecido como wavelet de Haar) e posteriormente o filtro de Daubechies db3, que correspondem, respectivamente, aos filtros de ordem 2 e 6 . Os resultados das Seções 7.2 e 7.3 foram obtidos, respectivamente, decompondo-se as séries em 7 e 9 níveis.

\subsection{Resultados obtidos}

O resultado da decomposição DWPT é apresentado nas Figuras 7.1-7.3 para o caso da decomposição db1 e nas Figuras $7.4-7.6$ para o caso da decomposição db3. Além da decomposição DWPT (representada pela linha contínua azul), tais Figuras apresentam também, para efeitos de comparação, a decomposição DWT 
correspondente (representada pelos círculos vermelhos, interligados por uma linha contínua vermelha para melhor visualização). Os gráficos das Figuras 7.1-7.6 são apresentados em função da oitava $j$.

Como seria esperado, o espectro DWT está intrinsicamente relacionado com o espectro DWPT. Em todos os gráficos das respectivas decomposições, pode-se observar visualmente que um ponto do espectro DWT para uma determinada escala corresponde ao ponto médio dos respectivos pontos do espectro DWPT da mesma escala. Isso é ainda mais notório para os casos de decomposição db1 sem a presença de SRD (Figuras 7.1a, 7.2a e 7.3a), uma vez que em tais casos o espectro DWPT é aproximadamente plano para uma mesma escala de observação.

Entretanto, alguns resultados interessantes podem ser obtidos das informações adicionais fornecidas pelo espectro DWPT. Para o caso da decomposição db1, pode-se observar nas Figuras 7.1-7.3 que, ao contrário do que foi acima observado para o caso da decomposição das séries sem SRD, no caso das séries com SRD o espectro wavelet não permanece plano para uma mesma escala. Tal fato pode ser utilizado em um mecanismo de detecção de presença de SRD. Nesse caso, uma vez detectada a presença de SRD, a utilização do espectro DWPT pode ser também vantajosa na identificação das componentes do espectro em que há presença de SRD.

As Figuras 7.7 e 7.8 demonstram, em especial para o caso que utiliza o filtro db3, como pode ser facilmente observada a variação no espectro wavelet das componentes SRD acrescentadas em uma mesma realização. Isso porque, ao contrário do espectro DWT, onde a componente SRD influencia apenas levemente alguns poucos pontos do espectro, no caso do espectro DWPT uma grande quantidade de pontos é influenciada, permitindo uma identificação mais fácil da frequencia central da componente SRD (no caso de ser utilizado algum método para atenuar tal componente antes da estimação do valor de $H$ ).

Finalmente, conforme já descrito na Seção 4.3, o método de Abry-Veitch para estimação de $H$ realiza uma ponderação dos coeficientes da análise DWT para obter a reta ajustada ao espectro, sendo que as escalas mais baixas recebem um peso maior em tal ponderação. A utilização da DWPT em substituição à DWT em tal método poderia simplificá-lo uma vez que naturalmente as escalas mais baixas dispõem de mais pontos, não havendo a necessidade da ponderação. 


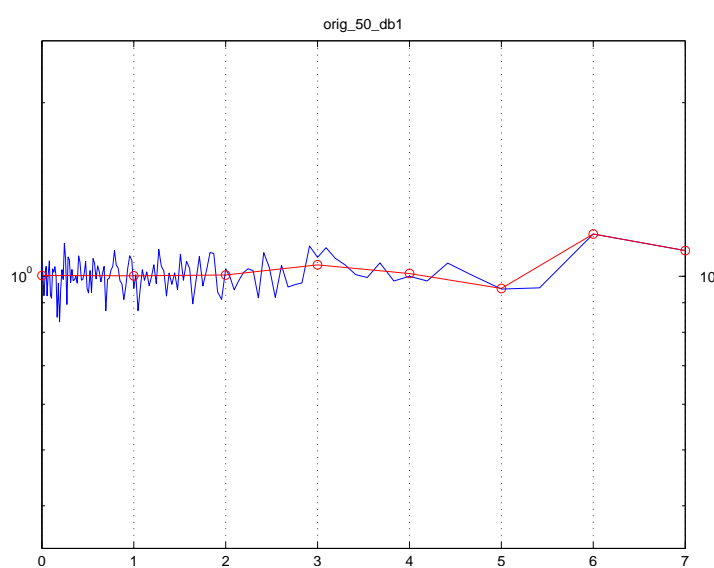

(a) Séries originais sem SRD

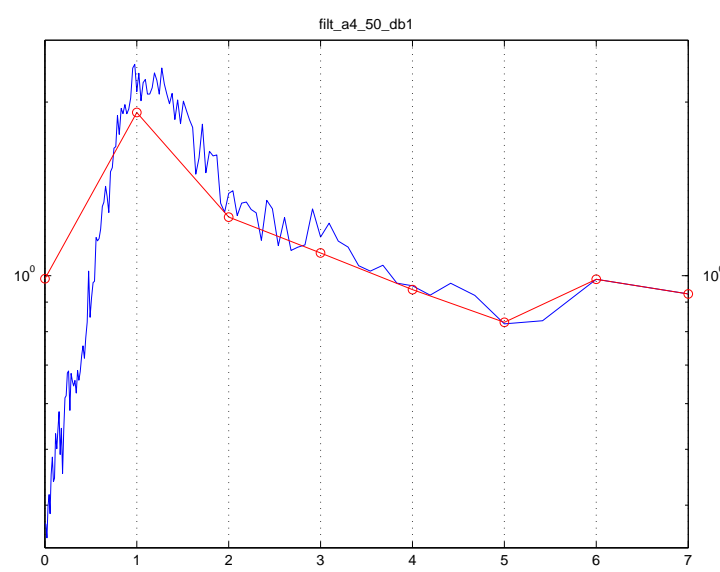

(c) Séries com SRD - $f_{0} \approx 0,4$

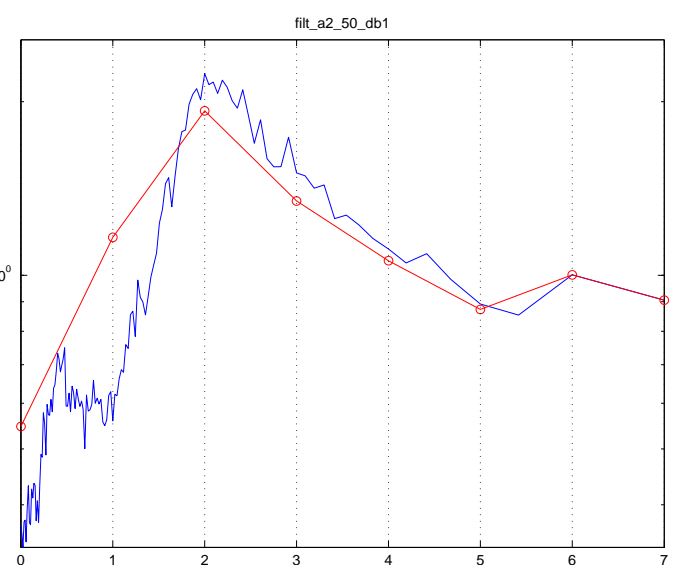

(b) Séries com SRD - $f_{0} \approx 0,2$

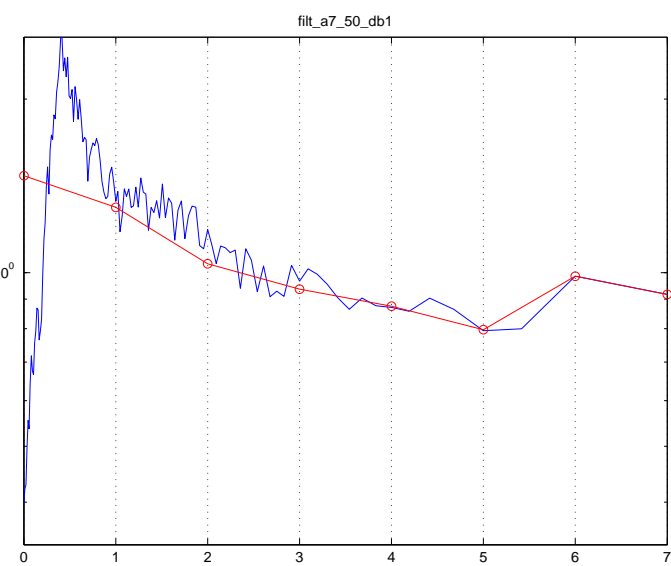

(d) Séries com SRD - $f_{0} \approx 0,7$

Figura 7.1: Comparação do espectro wavelet obtido pela decomposição DWT e pela decomposição DWPT com filtro db1 $-H=0,5$. 


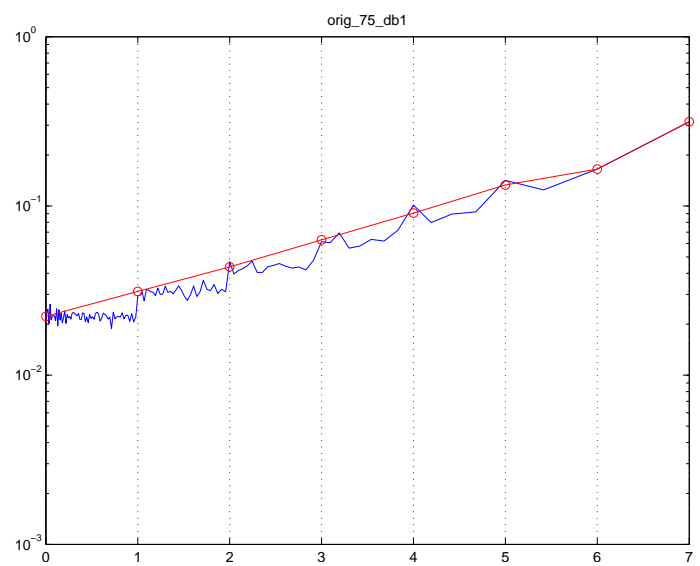

(a) Séries originais sem SRD

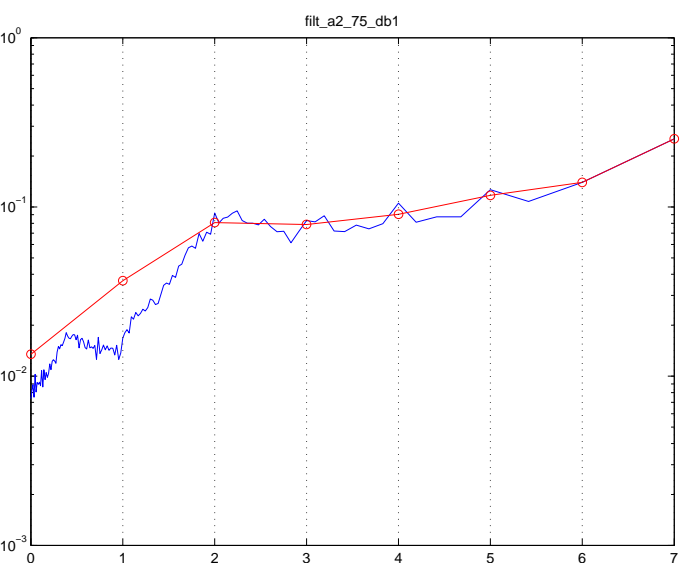

(b) Séries com SRD - $f_{0} \approx 0,2$

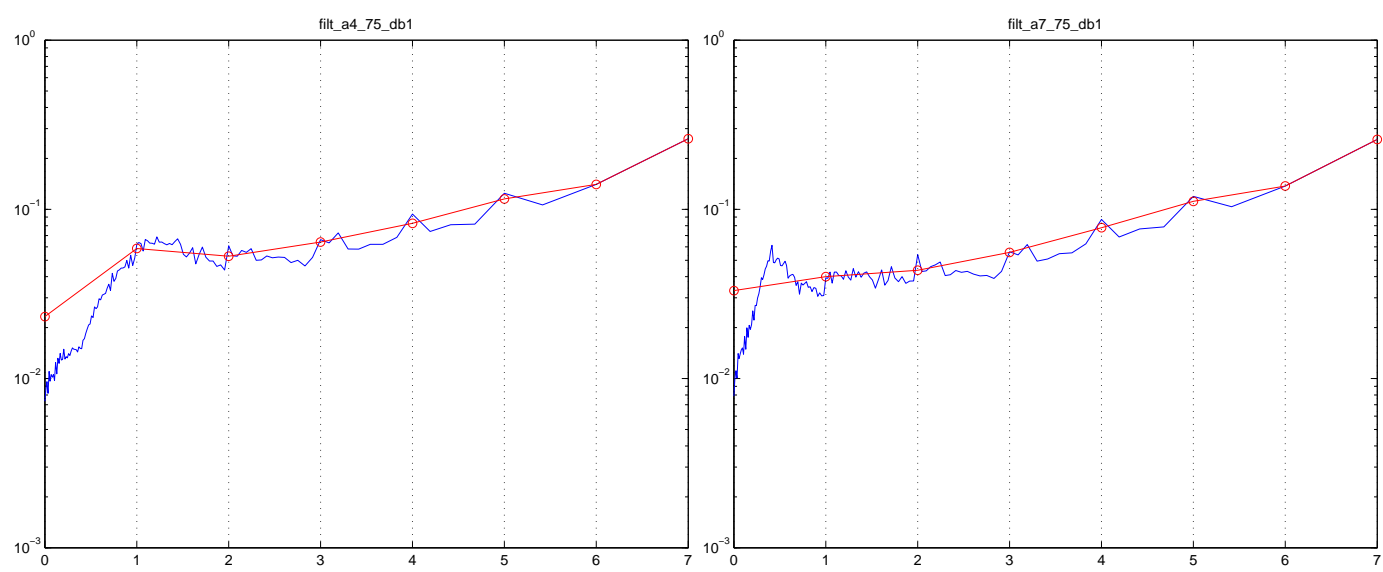

(c) Séries com SRD - $f_{0} \approx 0,4$

(d) Séries com SRD - $f_{0} \approx 0,7$

Figura 7.2: Comparação do espectro wavelet obtido pela decomposição DWT e pela decomposição DWPT com filtro db1 $-H=0,75$. 


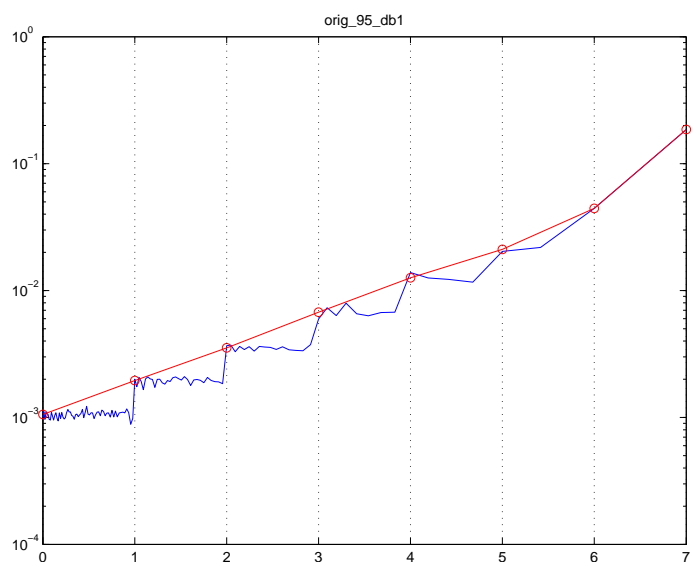

(a) Séries originais sem SRD

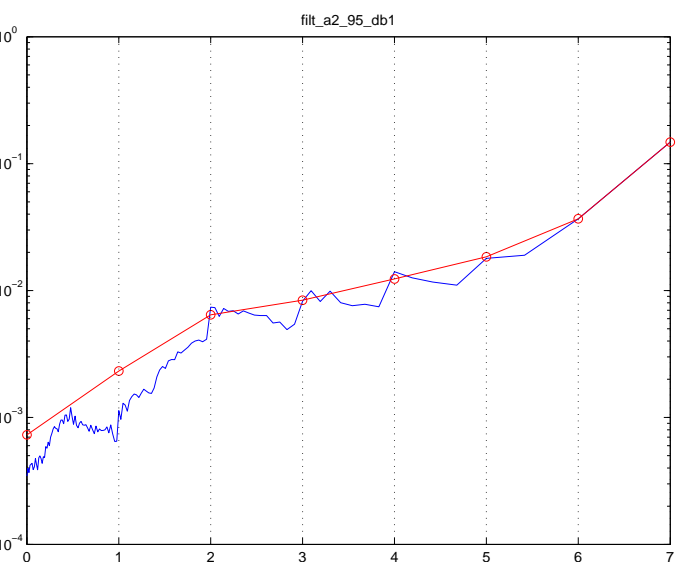

(b) Séries com SRD - $f_{0} \approx 0,2$

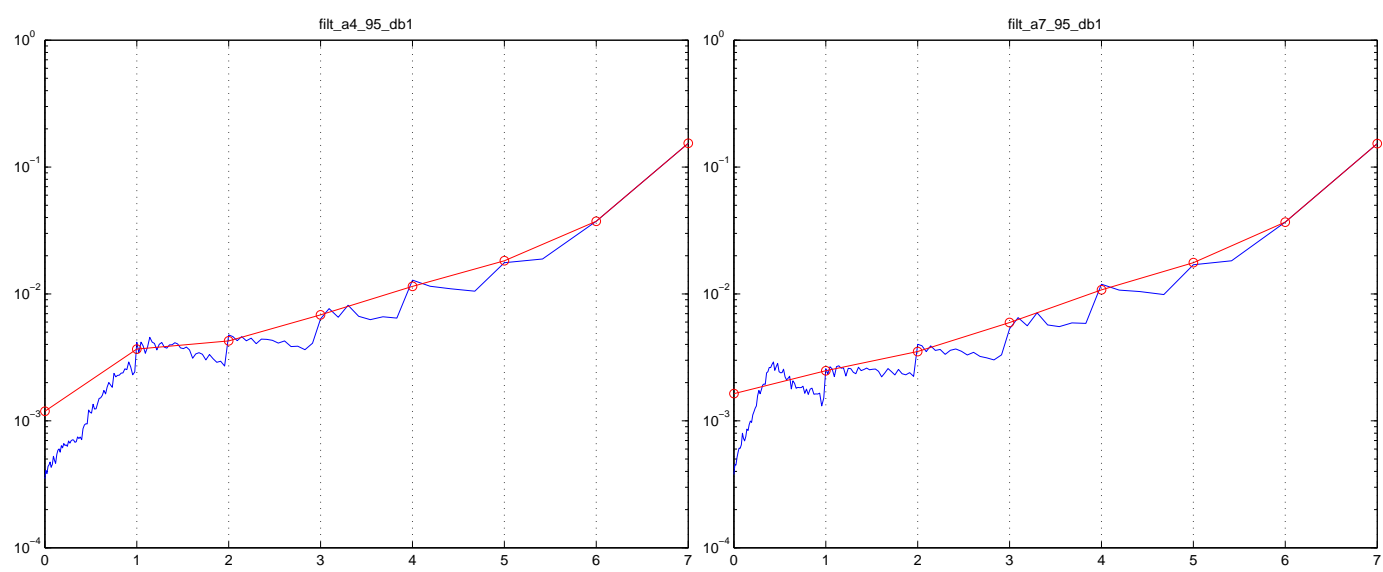

(c) Séries com SRD - $f_{0} \approx 0,4$

(d) Séries com SRD - $f_{0} \approx 0,7$

Figura 7.3: Comparação do espectro wavelet obtido pela decomposição DWT e pela decomposição DWPT com filtro db1 $-H=0,95$. 


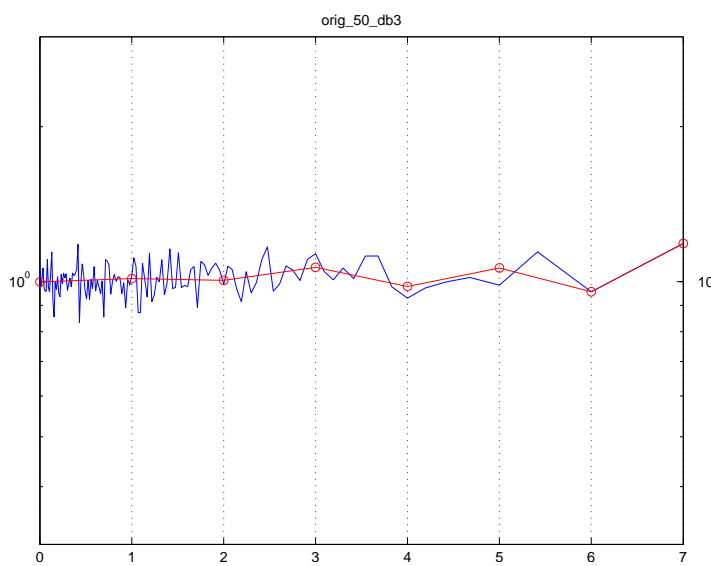

(a) Séries originais sem SRD

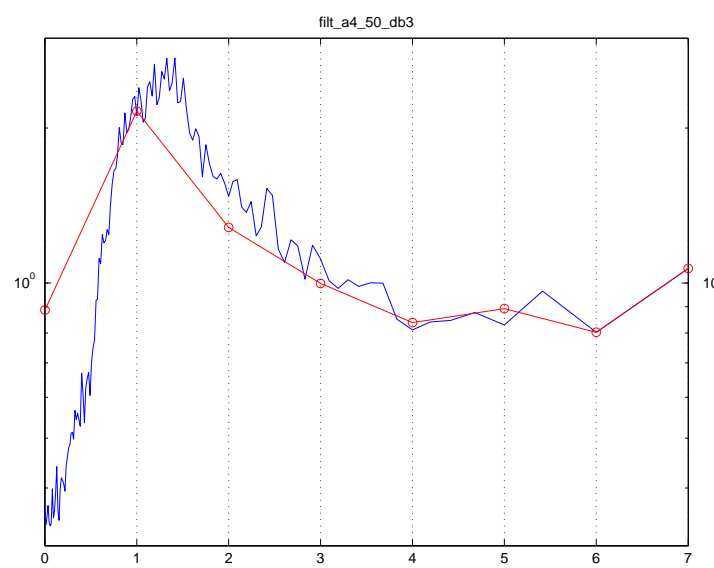

(c) Séries com SRD - $f_{0} \approx 0,4$

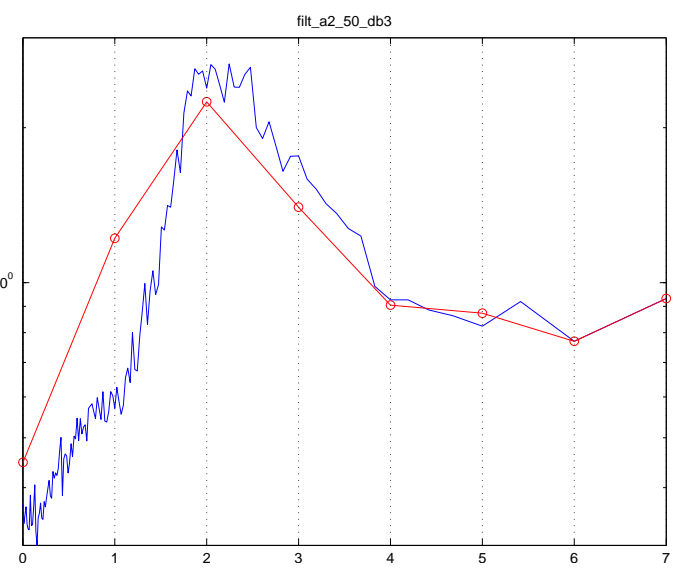

(b) Séries com SRD - $f_{0} \approx 0,2$

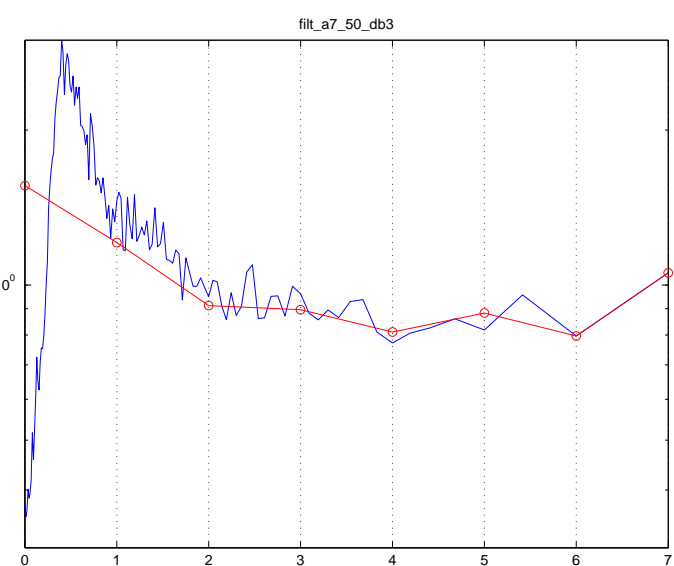

(d) Séries com SRD - $f_{0} \approx 0,7$

Figura 7.4: Comparação do espectro wavelet obtido pela decomposição DWT e pela decomposição DWPT com filtro db3 $-H=0,5$. 


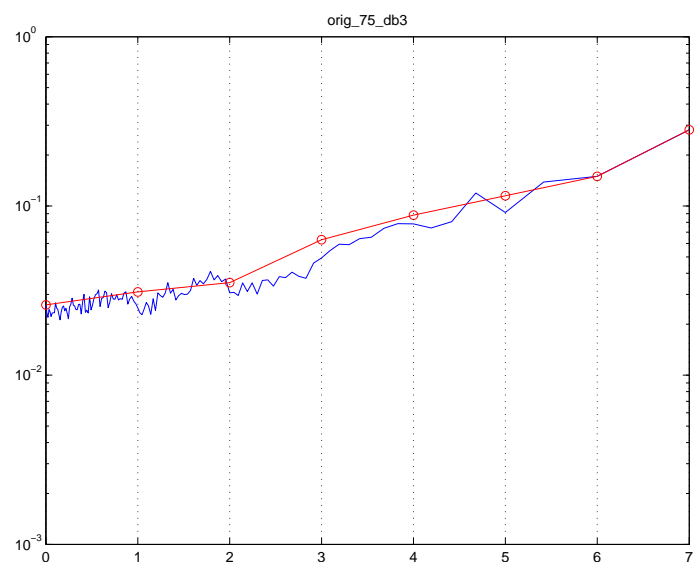

(a) Séries originais sem SRD

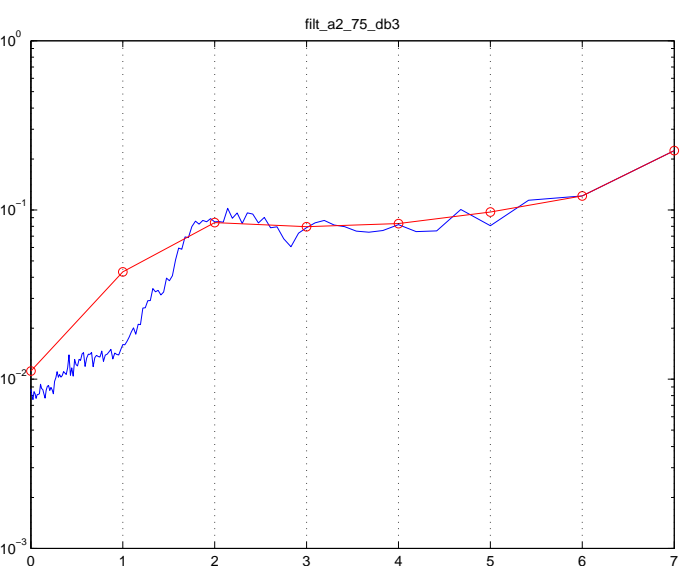

(b) Séries com SRD - $f_{0} \approx 0,2$

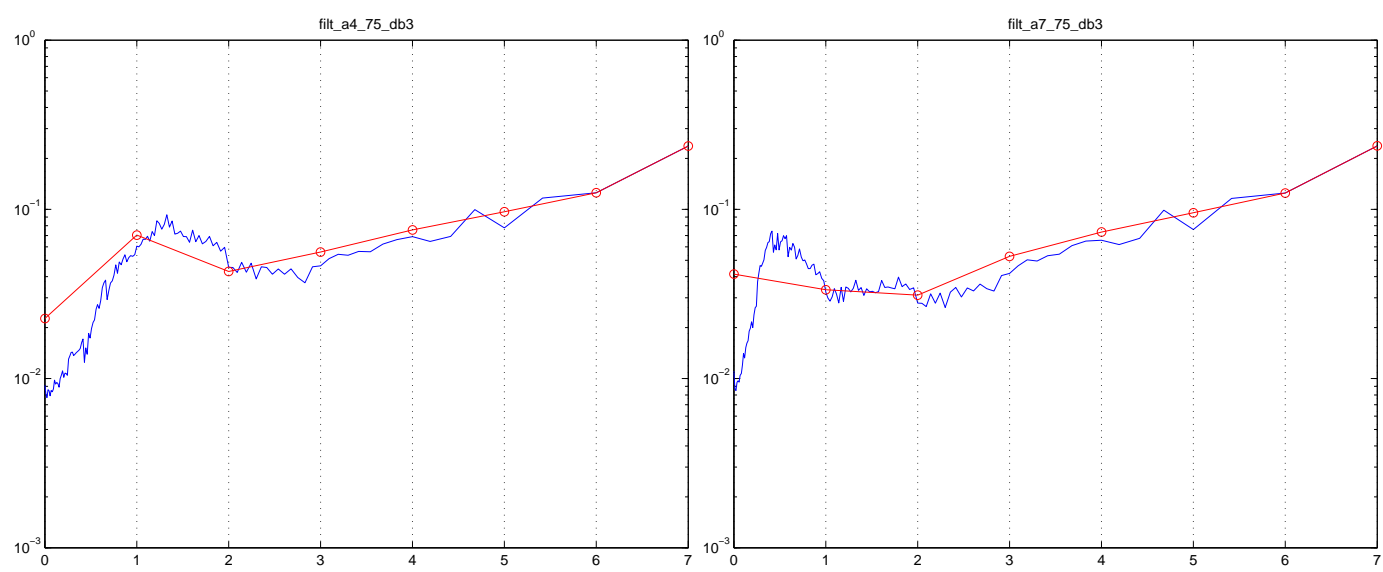

(c) Séries com SRD - $f_{0} \approx 0,4$

(d) Séries com SRD - $f_{0} \approx 0,7$

Figura 7.5: Comparação do espectro wavelet obtido pela decomposição DWT e pela decomposição DWPT com filtro db3 $-H=0,75$. 


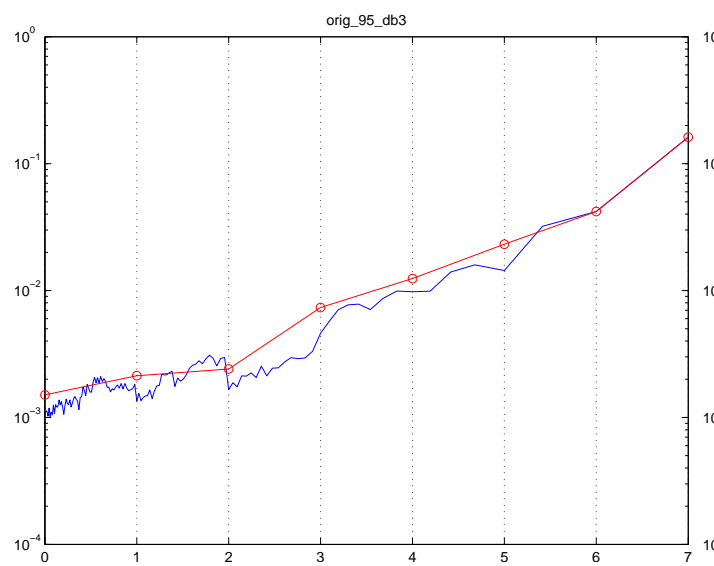

(a) Séries originais sem SRD

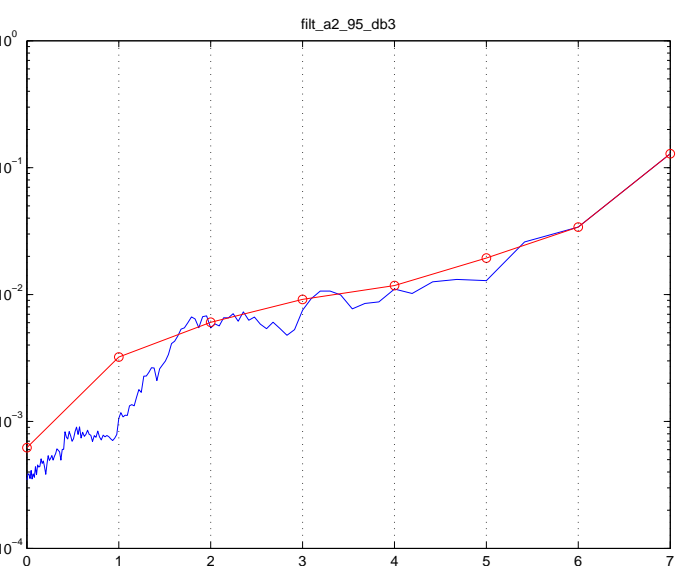

(b) Séries com SRD - $f_{0} \approx 0,2$

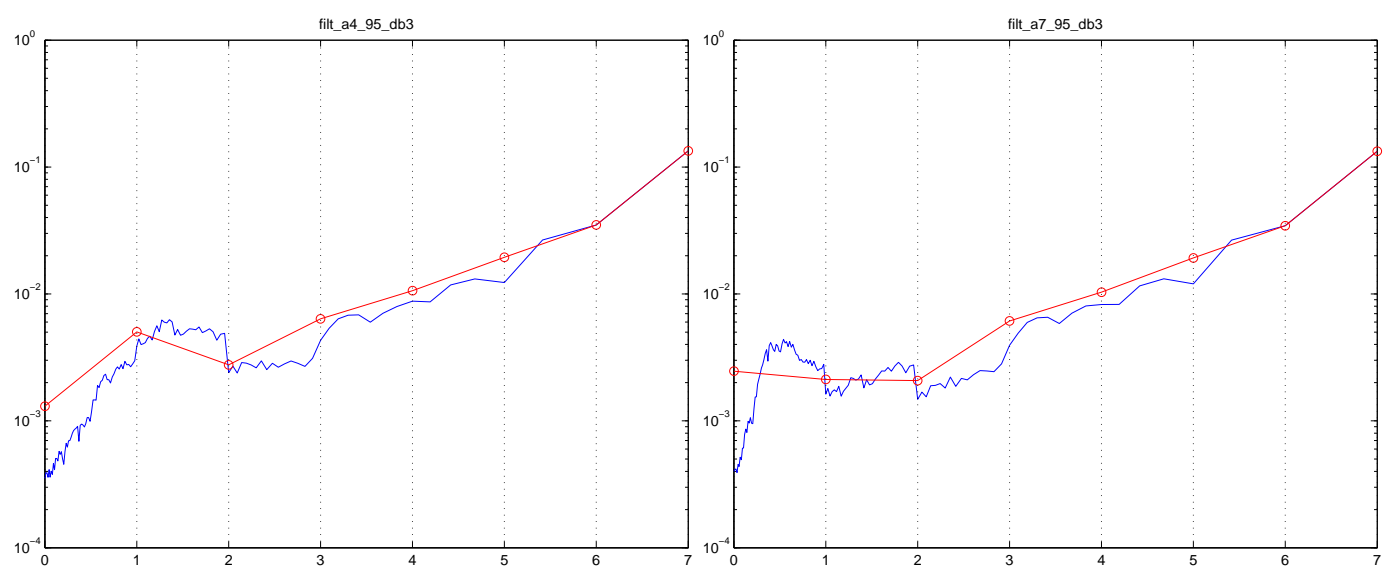

(c) Séries com SRD - $f_{0} \approx 0,4$

(d) Séries com SRD - $f_{0} \approx 0,7$

Figura 7.6: Comparação do espectro wavelet obtido pela decomposição DWT e pela decomposição DWPT com filtro db3 $-H=0,95$. 


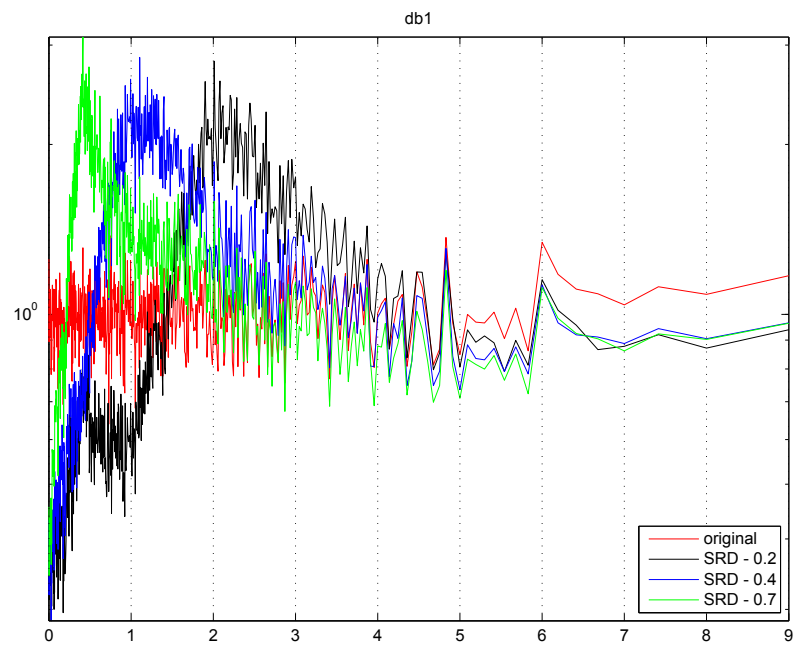

(a) $H=0,5$

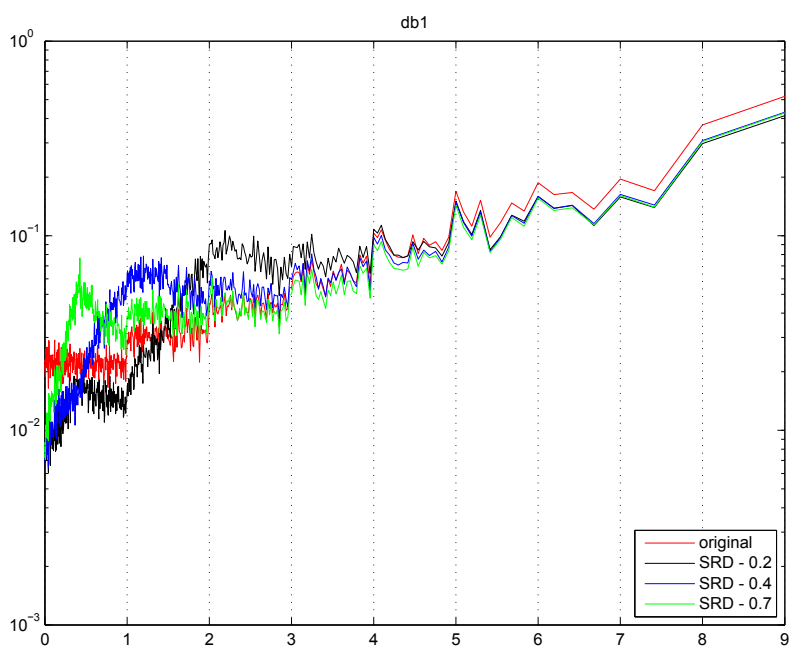

(b) $H=0,75$

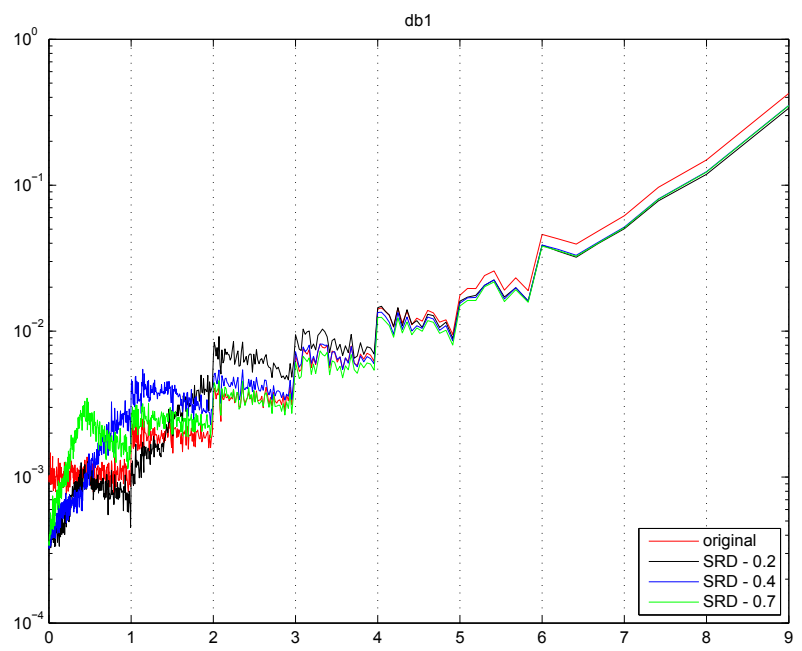

(c) $H=0,95$

Figura 7.7: Comparação dos espectros wavelet para os casos sem e com componentes SRD - decomposição DWPT com filtro db1. 


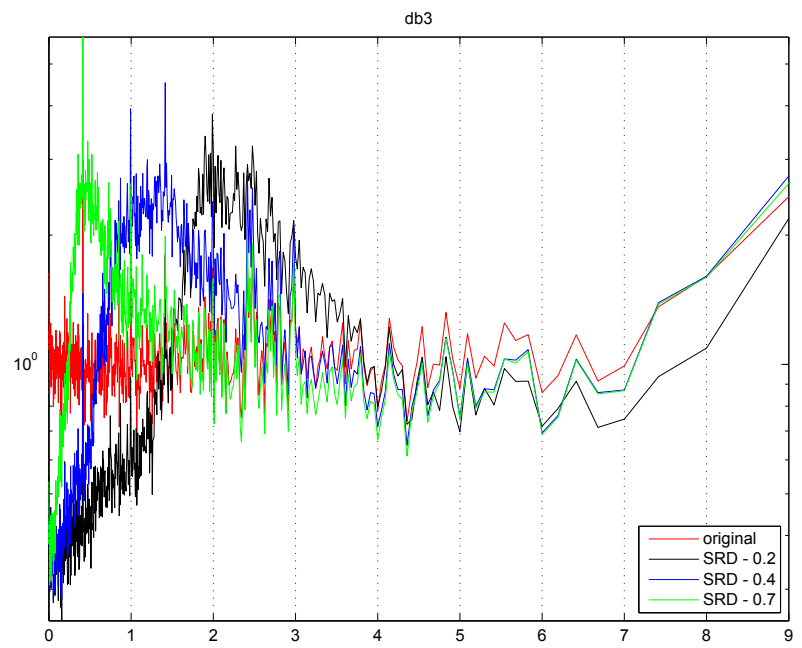

(a) $H=0,5$

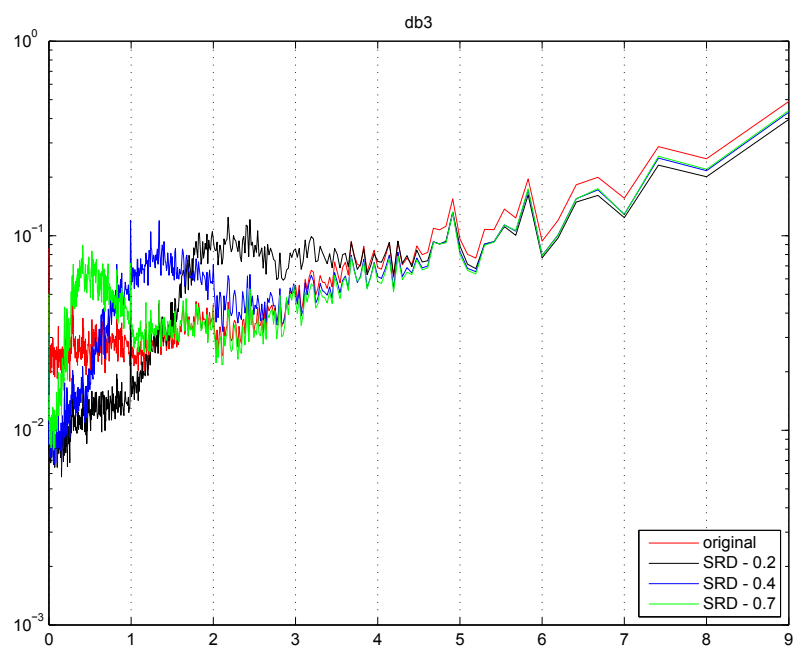

(b) $H=0,75$

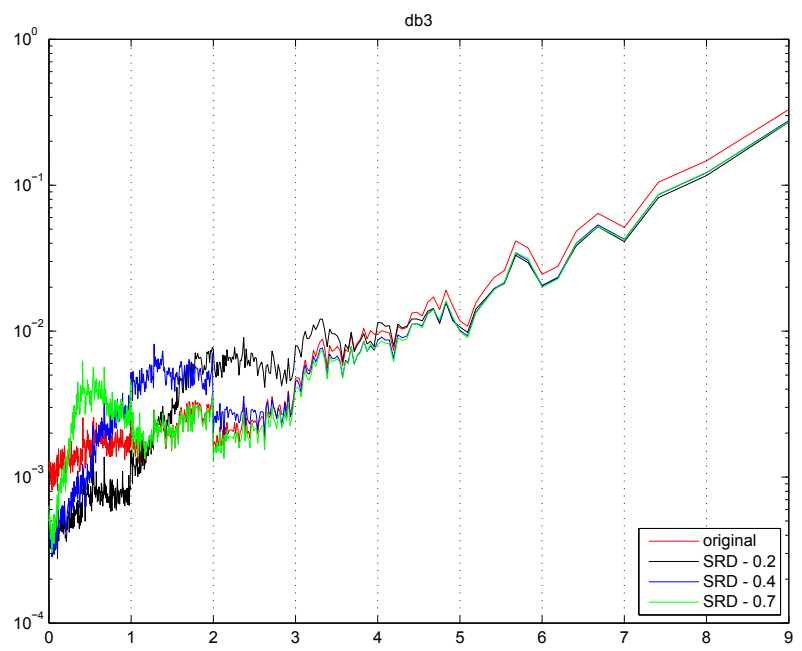

(c) $H=0,95$

Figura 7.8: Comparação dos espectros wavelet para os casos sem e com componentes SRD - decomposição DWPT com filtro db3. 


\subsection{Exploração dos resultados}

Dando sequência à ideia exposta na Seção 7.2 de utilização do espectro DWPT em substituição ao espectro DWT para estimação do valor de $H$, esta Seção visa analisar os resultados obtidos através de tal substituição, comparando as retas ajustadas ao espectro wavelet obtidos de árvores DWPT diversas.

Tal estudo foi realizado primeiramente analisando-se os espectros wavelet, da série original sem SRD, obtidos das seguintes formas:

- árvore DWPT com coeficientes de nível 9

- árvore DWPT com coeficientes de nível 6

- árvore DWPT com coeficientes da melhor árvore, segundo critério de entropia de Shannon

- árvore DWT

- árvore DWT "inversa", correspondente aos coeficientes DWPT simétricos aos coeficientes DWT, conforme ilustrado na Figura 7.9.

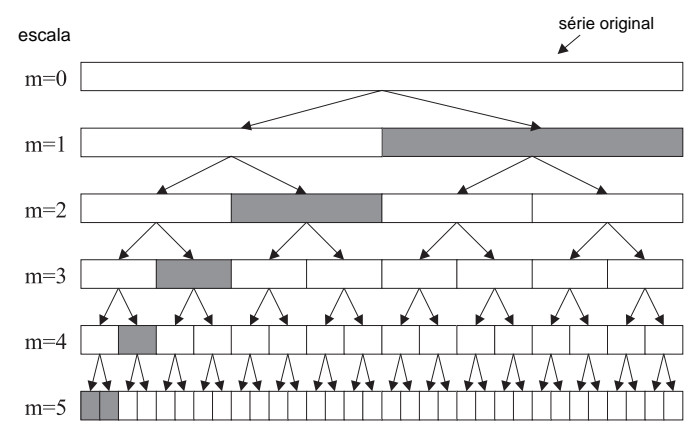

(a) Árvore DWT.

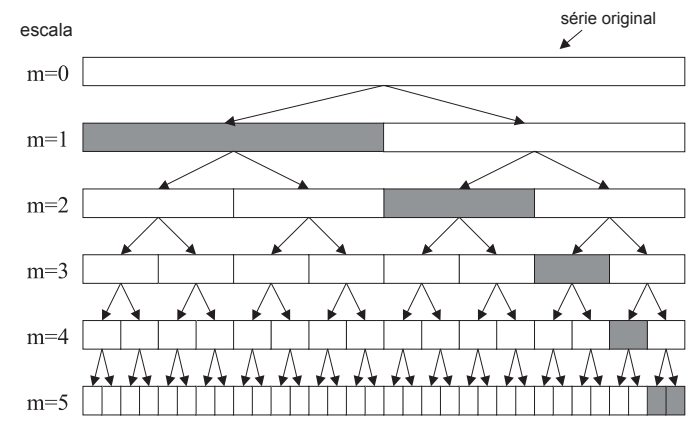

(b) Árvore DWT "inversa".

Figura 7.9: Comparação entre as árvore da decomposição DWT convencional e árvore da deconposição DWT "inversa".

A Figura 7.10a demonstra, em uma visualização 3D, a variância dos coeficientes da decomposição DWPT para uma série com $H=0,5$ e sem componentes SRD. Cada uma das linhas sobre tal gráfico corresponde ao conjunto de coeficientes necessários para formação de um dos diversos espectros wavelet acima enumerados. A Figura 7.10b ilustra, além dos respectivos espectros wavelet já citados, as retas ajustadas a cada um de tais espectros. De forma a aprofundar essa análise para o caso de componentes SRD estarem presentes na série em análise, a Figura 7.10c apresenta o resultado análogo ao apresentado na Figura 7.10a, porém com a adição de uma componente SRD à série estudada. Conforme 
pode ser observado, há uma sensível alteração no gráfico correspondente. De forma complementar, a Figura 7.10d apresenta os diversos espectros wavelet para a série com componente SRD. Neste caso, além das retas ajustadas para cada um dos espectros presentes, foi adicionada uma reta de referência correspondente ao ajuste obtido para o caso sem SRD utilizando-se a árvore DWT (vide Figura $7.10 b)$.

As Figuras 7.11 e 7.12 são análogas à Figura 7.10, mas para $H=0,75$ e $H=$ 0,95, respectivamente. De forma semelhante, as Figuras 7.13-7.15 são análogas às Figuras 7.10-7.12, uma vez que tais Figuras correspondem à análise efetuada utilizando-se a filtragem db3 na decomposição DWPT, e não a filtragem db1.

Analisando-se apenas qualitativamente os resultados obtidos, é possível observar que, em todos os casos estudados, o ajuste feito ao espectro pela árvore DWT para o caso de série com componente SRD foi o que mais se aproximou da reta de referência, indicando que possivelmente tal árvore seja realmente a mais indicada para estudo de séries com SRD. Entretanto, um importante resultado decorrente também de tal análise é que o espectro wavelet pode sofrer uma sensível variação dependendo da árvore escolhida, sendo que a escolha apropriada pode, inclusive, eliminar os efeitos decorrentes da componente SRD presentes em tais séries. Tal estudo, entretanto, não pertence ao escopo deste trabalho. 


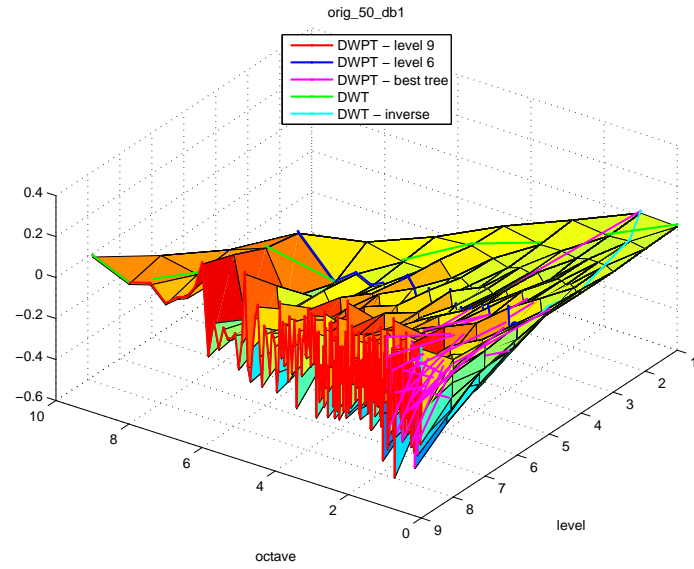

(a) Coeficientes para formação do espectro wavelet - Série original sem SRD

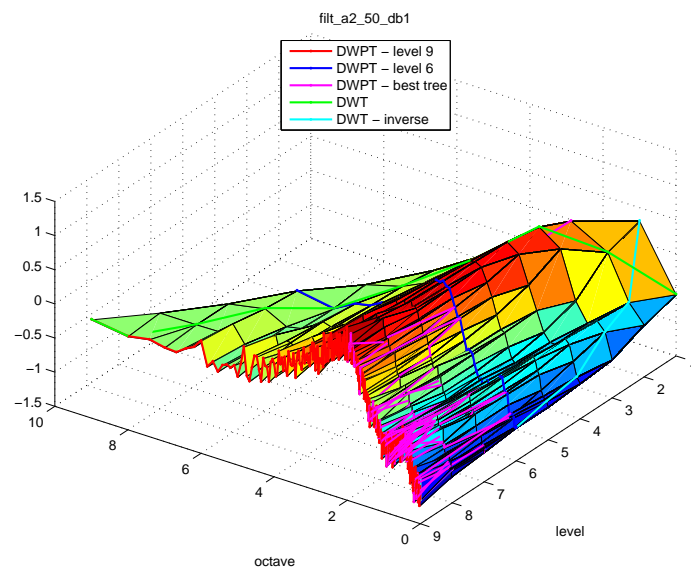

(c) Coeficientes para formação do espectro wavelet - Série com SRD $\left(f_{0} \approx 0,2\right)$

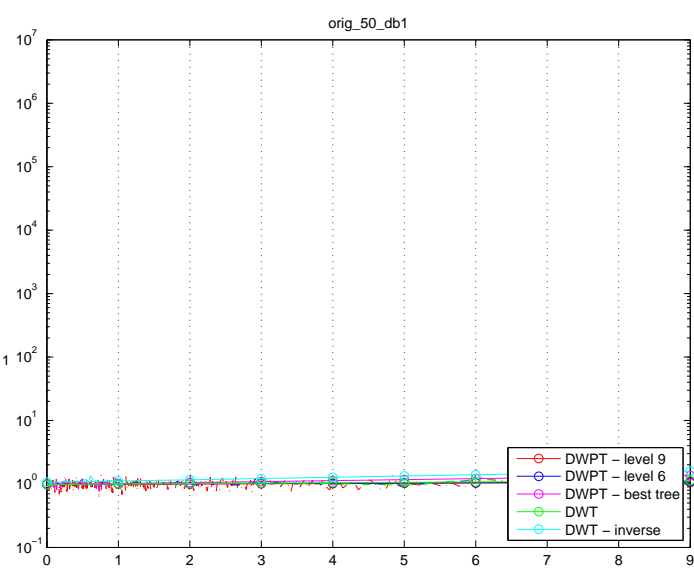

(b) Comparação entre espectros wavelet Série original sem SRD

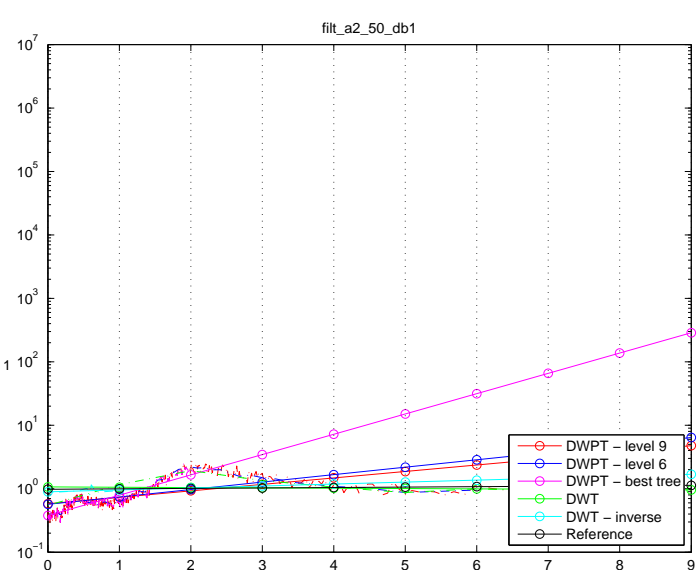

(d) Comparação entre espectros wavelet Série com $\operatorname{SRD}\left(f_{0} \approx 0,2\right)$

Figura 7.10: Comparação dos espectros wavelet para estimação do parâmetro $H$ - decomposição DWPT com filtro db1 de séries com $H=0,5$. 


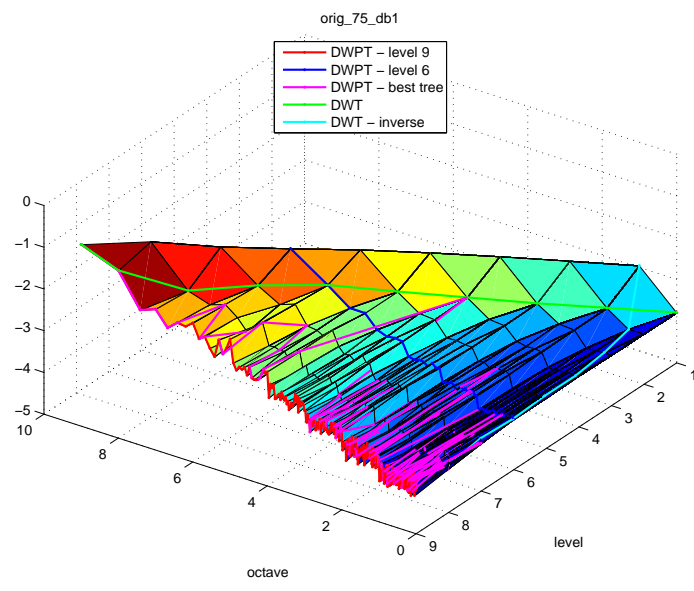

(a) Coeficientes para formação do espectro wavelet - Série original sem SRD

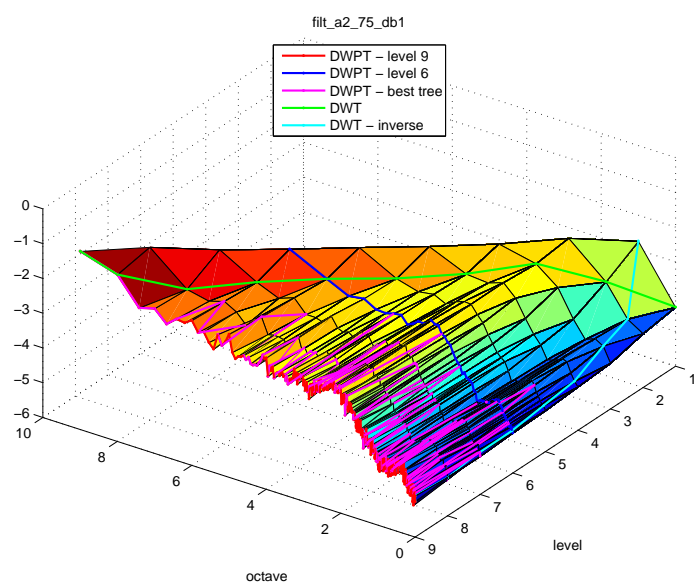

(c) Coeficientes para formação do espectro wavelet - Série com $\operatorname{SRD}\left(f_{0} \approx 0,2\right)$

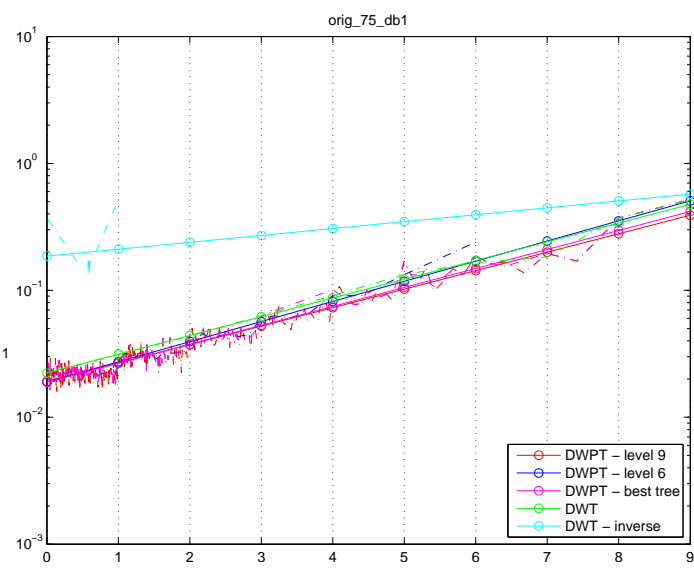

(b) Comparação entre espectros wavelet Série original sem SRD

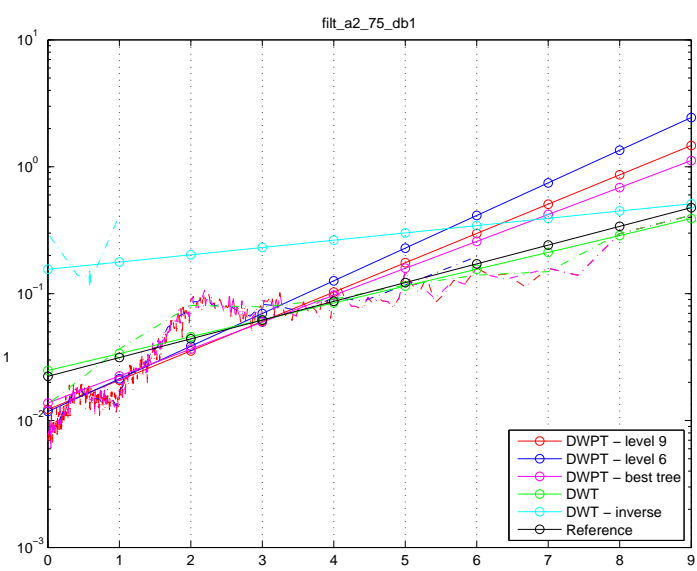

(d) Comparação entre espectros wavelet Série com $\operatorname{SRD}\left(f_{0} \approx 0,2\right)$

Figura 7.11: Comparação dos espectros wavelet para estimação do parâmetro $H$ - decomposição DWPT com filtro db1 de séries com $H=0,75$. 


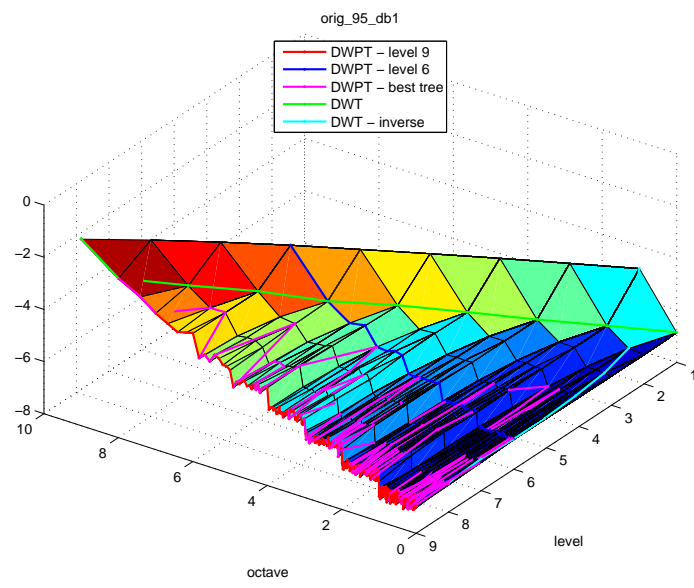

(a) Coeficientes para formação do espectro wavelet - Série original sem SRD

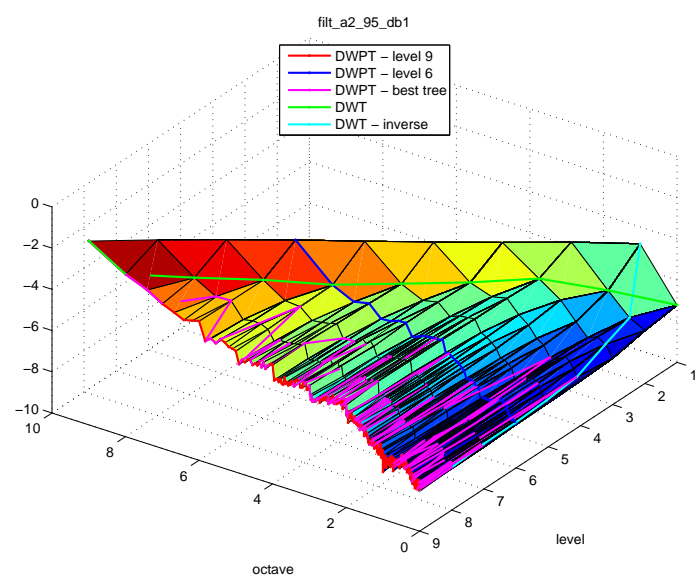

(c) Coeficientes para formação do espectro wavelet - Série com $\operatorname{SRD}\left(f_{0} \approx 0,2\right)$

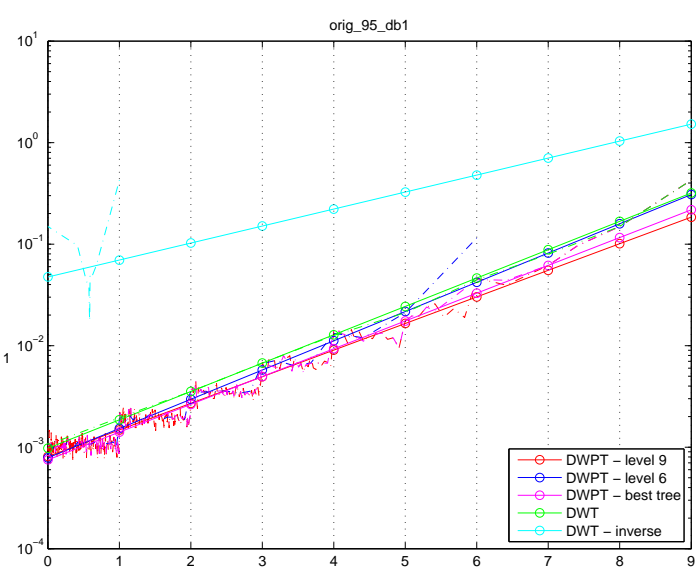

(b) Comparação entre espectros wavelet Série original sem SRD

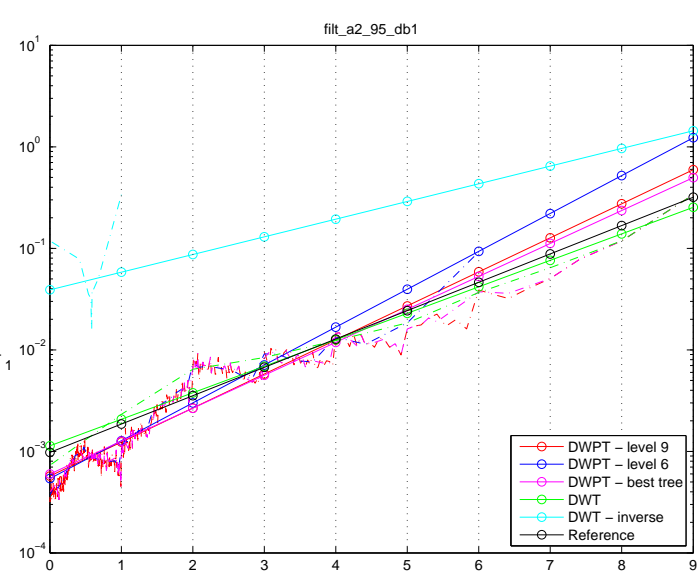

(d) Comparação entre espectros wavelet Série com $\operatorname{SRD}\left(f_{0} \approx 0,2\right)$

Figura 7.12: Comparação dos espectros wavelet para estimação do parâmetro $H$ - decomposição DWPT com filtro db1 de séries com $H=0,95$. 


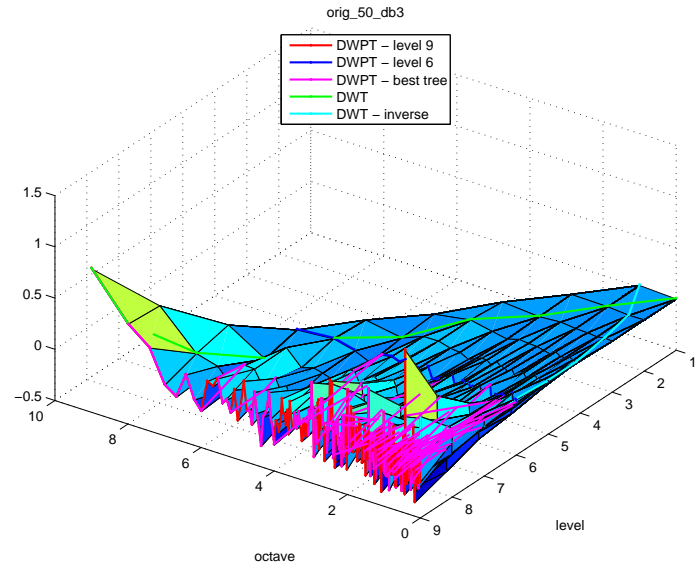

(a) Coeficientes para formação do espectro wavelet - Série original sem SRD

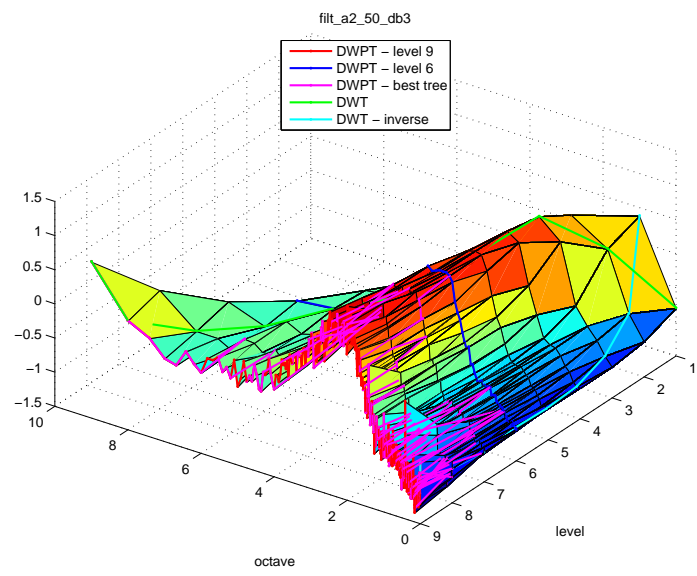

(c) Coeficientes para formação do espectro wavelet - Série com SRD $\left(f_{0} \approx 0,2\right)$

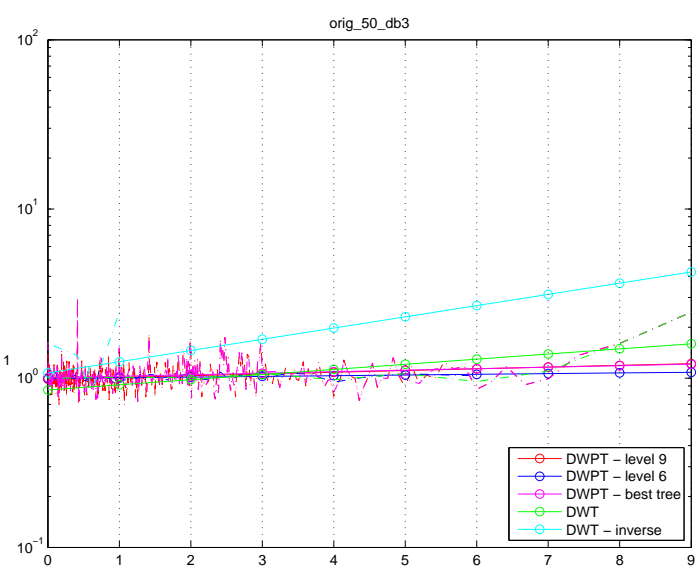

(b) Comparação entre espectros wavelet Série original sem SRD

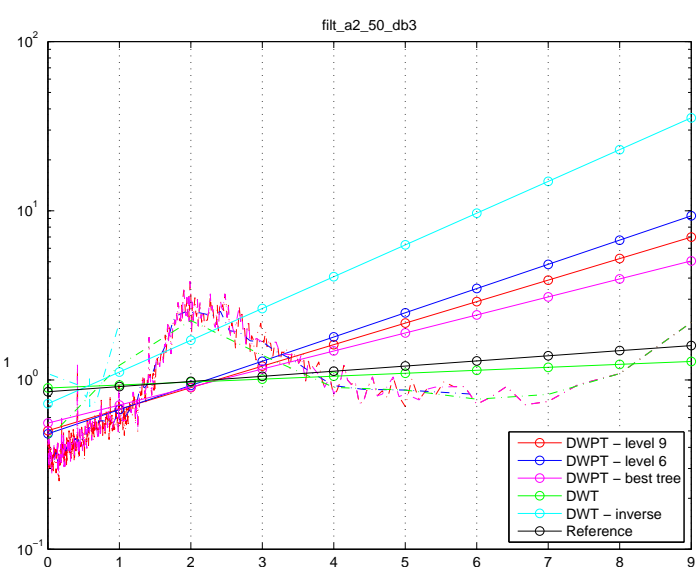

(d) Comparação entre espectros wavelet Série com $\operatorname{SRD}\left(f_{0} \approx 0,2\right)$

Figura 7.13: Comparação dos espectros wavelet para estimação do parâmetro $H$ - decomposição DWPT com filtro db3 de séries com $H=0,5$. 


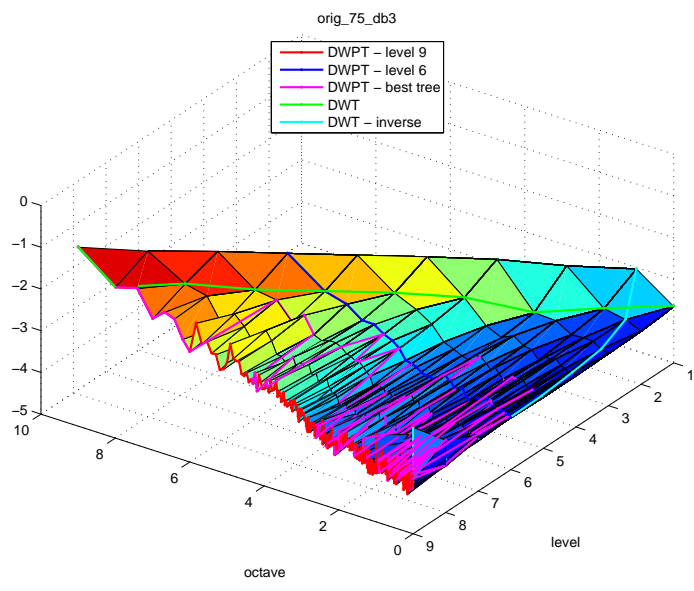

(a) Coeficientes para formação do espectro wavelet - Série original sem SRD

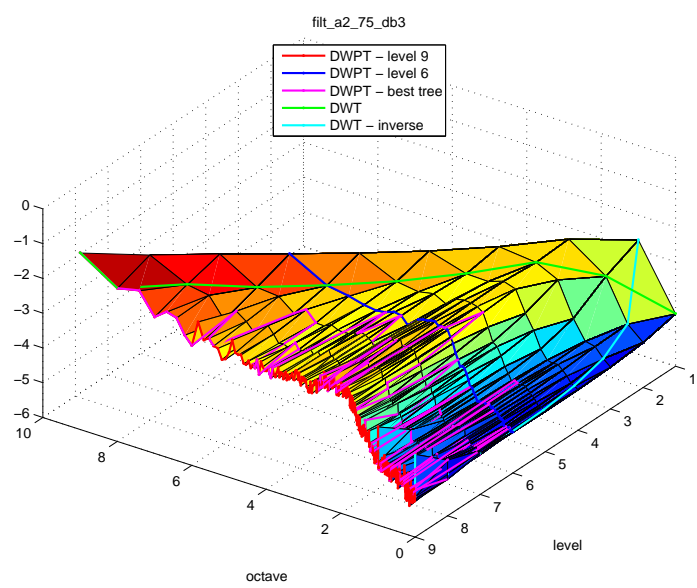

(c) Coeficientes para formação do espectro wavelet - Série com $\operatorname{SRD}\left(f_{0} \approx 0,2\right)$

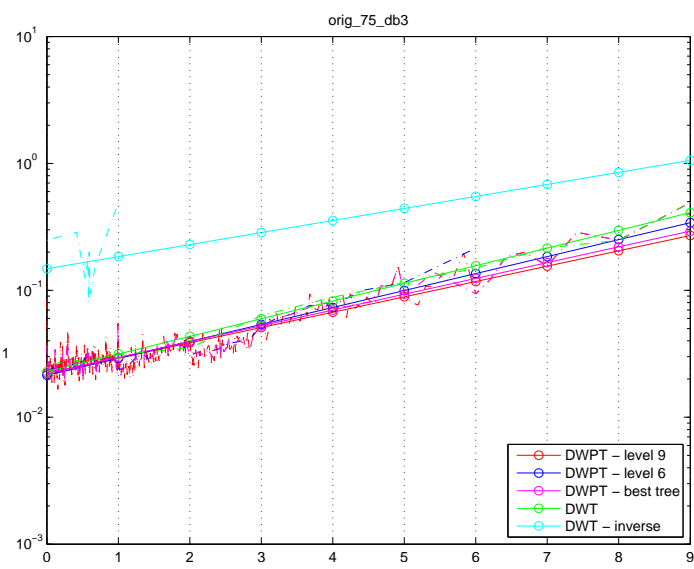

(b) Comparação entre espectros wavelet Série original sem SRD

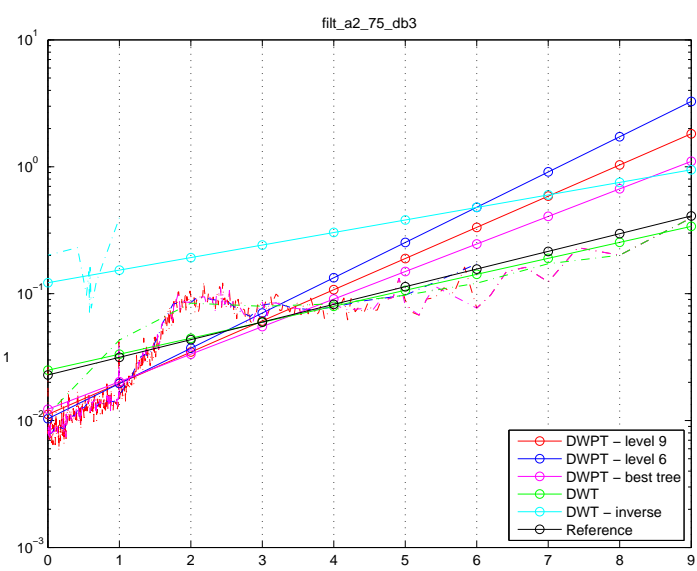

(d) Comparação entre espectros wavelet Série com $\operatorname{SRD}\left(f_{0} \approx 0,2\right)$

Figura 7.14: Comparação dos espectros wavelet para estimação do parâmetro $H$ - decomposição DWPT com filtro db3 de séries com $H=0,75$. 


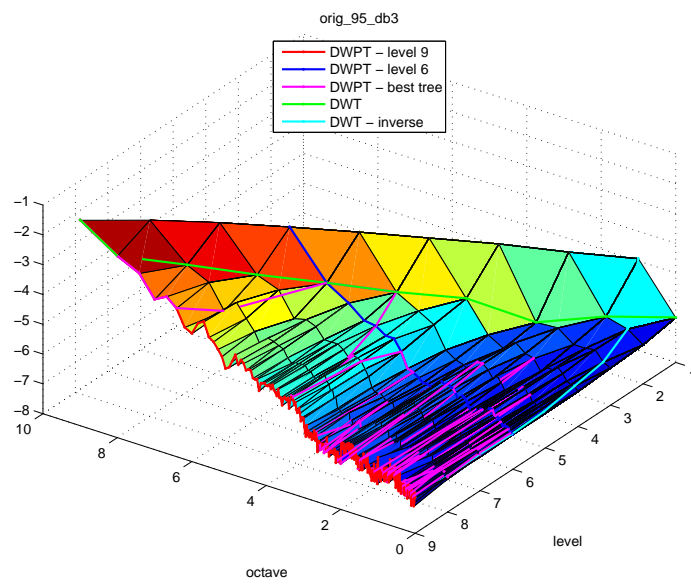

(a) Coeficientes para formação do espectro wavelet - Série original sem SRD

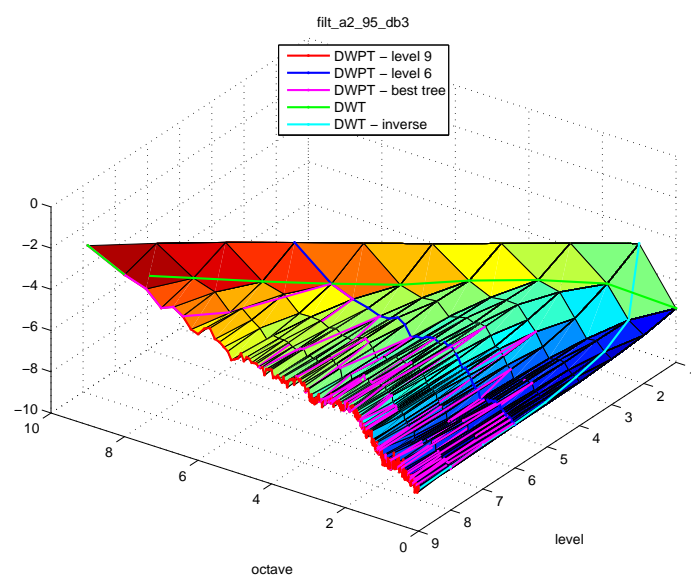

(c) Coeficientes para formação do espectro wavelet - Série com $\operatorname{SRD}\left(f_{0} \approx 0,2\right)$

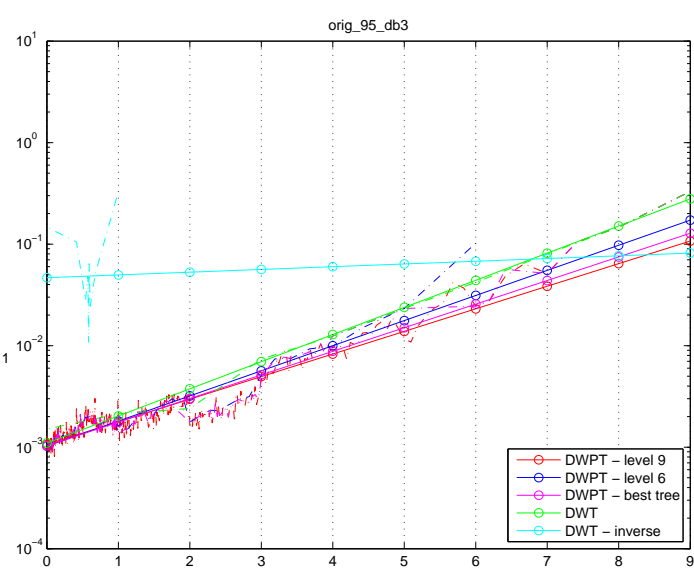

(b) Comparação entre espectros wavelet Série original sem SRD

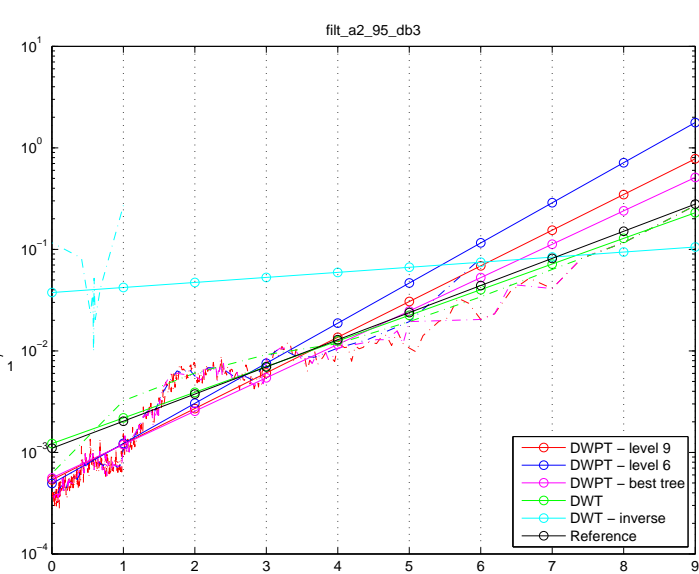

(d) Comparação entre espectros wavelet Série com $\operatorname{SRD}\left(f_{0} \approx 0,2\right)$

Figura 7.15: Comparação dos espectros wavelet para estimação do parâmetro $H$ - decomposição DWPT com filtro de séries com $H=0,95$. 


\subsection{Síntese dos resultados}

A Tabela 7.1 apresenta uma síntese dos principais resultados expostos no Capítulo 7.

Tabela 7.1: Resultados do Capítulo 7.

\begin{tabular}{|c|l|}
\hline \hline Resultado & Descrição \\
\hline \hline 1 & $\begin{array}{l}\text { Possibilidade de identificar, através do espectro wavelet obtido pela decompo- } \\
\text { sição DWPT com filtragem db1, se a série analisada possui componentes SRD } \\
\text { ou não }\end{array}$ \\
\hline 2 & $\begin{array}{l}\text { Decomposição DWPT possibilita obter um espectro wavelet mais "rico", per- } \\
\text { mitindo, inclusive, determinar com maior precisão a componente SRD eventu- } \\
\text { almente presente na série em estudo }\end{array}$ \\
\hline 3 & $\begin{array}{l}\text { Diversas árvores DWPT distintas podem ser utilizadas para determinação do } \\
\text { espectro wavelet, sendo que a escolha da melhor árvore, segundo alguma métrica } \\
\text { a ser definida, pode resultar em um espectro wavelet tal que componentes SRD } \\
\text { não influenciem na determinação de } H\end{array}$ \\
\hline \hline
\end{tabular}




\section{Proposta de mecanismo de estimação}

O estudo realizado das características e limitações do algoritmo de Abry-Veitch para estimação do parâmetro de Hurst, bem como o estudo da DWPT como alternativa para estimação do espectro wavelet, geraram resultados relevantes que foram devidamente sumarizados nos Capítulos 6 e 7 , respectivamente.

Em conformidade com o objetivo inicial de propor alternativas para que possa ser realizada uma estimação em tempo real das características LRD do tráfego na presença de componentes SRD, o conjunto de resultados obtidos é completo o suficiente para permitir que seja feita uma proposta de mecanismo em tempo real de estimação de $H$. Com esse objetivo, os principais resultados da análise exploratória realizada são revisitados na Seção 8.1. De posse de tais resultados, a Seção 8.2 apresenta a proposta de tal mecanismo de estimação, enquanto a Seção 8.3 enumera alguns pontos a serem ainda esclarecidos para a implementação do mecanismo.

\subsection{Fundamentação experimental}

A análise efetuada da variação dos parâmetros do algoritmo de Abry-Veitch para estimação em diversas condições de teletráfego evidenciou que (1) a utilização do parâmetro $j_{\text {min }}=j_{\text {min }}^{E Q M}$ efetivamente produz uma correção do viés do estimador, em troca de maior dispersão das estimativas, no caso de componentes SRD estarem presentes no teletráfego. Entretanto, os diversos gráficos gerados de tal comportamento em função do parâmetro $N$ podem ser utilizados para determinar o valor de $N$ necessário para que a dispersão do estimador tenha seu valor limitado a um valor pré-estabelecido. Adicionalmente, os gráficos gerados dos espectros wavelet, para $N$ suficientemente grande, possibilitaram (2) a identificação de região do espectro em que não é observada influência das componentes SRD. Mais ainda, também para $N$ suficientemente grande, foi observado que (3) 
o parâmetro $j_{\min }^{E Q M}$ assume valores próximos ao valor da escala de início da região do espectro em que não se observa influência de SRD, escala esta aqui denominada de "escala de transição". De forma complementar, foi observado que (4) o crescimento de $j_{\min }^{E Q M}$, para valores de $N$ ainda maiores, é mais acelerado do que o crescimento da escala de transição.

Com relação ao estudo realizado para análise do comportamento do algoritmo de Abry-Veitch na presença de pontos de mudança de componentes SRD, foi analisada (5) a questão do tamanho da janela de estimação em casos de estimação em tempo real, sendo observado que, enquanto é desejável a utilização da maior janela possível para redução da dispersão do estimador, tal escolha compromete o estimador no caso de transições das componentes LRD e SRD. Ainda com relação ao comportamento transitório do estimador, foi possível observar que (6) o espectro wavelet no caso de transições da componente LRD e no caso de transições da componente SRD têm comportamento nitidamente distintos.

Uma outra alternativa, a ser considerada para a implementação de um sistema de estimação em tempo real, é (7) a utilização exclusiva do parâmetro $j_{\min }=1$, devido à baixa dispersão das estimativas; como o comportamento do estimador nesse caso é altamente previsível (conforme gráficos obtidos), o alto viés ocasionado por tal esquema pode ser facilmente compensado, bastando um conhecimento mínimo, da série em que é efetuada a estimação, com relação às componentes SRD eventualmente presentes.

Com relação aos estudos realizados com a transformada DWPT para obtenção do espectro wavelet, observou-se que (8) é possível identificar em tal espectro, desde que obtido com a filtragem db1, se a série analisada possui componentes SRD ou não. Mais ainda, (9) o espectro wavelet mais "rico" obtido com a DWPT possibilita identificar, mesmo que de forma inicialmente grosseira através do método visual, as componentes SRD presentes na série analisada. De forma complementar, foi identificado que (10) o espectro wavelet obtido via DWPT pode ser utilizado diretamente, de forma alternativa ao espectro DWT, na estimação de $H$ pelo método de Abry-Veitch. Finalmente, observou-se que (11) a correta estimação do espectro wavelet, utilizando a DWPT pode estar condicionada à determinação da árvore DWPT "ideal”, segundo algum critério estabelecido, de forma que desconsidere as componentes SRD. 


\subsection{Mecanismo de estimação em tempo real}

Conforme os resultados (1)-(11) apresentados na Seção 8.1, o seguinte mecanismo de estimação em tempo real é proposto.

Conforme dados tabulados para os diversos casos estudados, dados o viés e a dispersão máximos desejados para uma determinada estimação, é possível determinar o valor de $N$ que deve ser atingido (cf. (1)) para satifazer as condições de viés e dispersão determinadas. Adicionalmente, enquanto o valor de $N$ não for atingido, é possivel realizar a estimação (cf. (7)) utilizando $j_{\min }=1$, estimação esta que seria devidamente corrigida com os dados tabulados, uma vez que se trata de um caso com comportamento muito bem definido de viés.

Entretanto, para que o passo acima possa ser executado, observa-se que é necessário um conhecimento prévio da existência ou não de componentes SRD no tráfego analisado, bem como uma estimativa inicial de $H$. A estimativa inicial de $H$ pode ser arbitrada e o seu valor correto ser obtido através de diversas iterações, desde que a determinação da existência de componentes SRD (cf. (8)) e a determinação relativamente precisa de tais componentes (cf. (9)) sejam realizadas através da análise DWPT.

Com relação ao tamanho da janela a ser utilizada na estimação em tempo real (cf. (5)), propõe-se um esquema de janelamento híbrido: uma janela de tamanho $N$ seria utilizada para realizar a estimação de $H$ propriamente dita. Esta janela, inicialmente de tamanho unitário, seria aumentada a cada nova amostra até chegar ao valor máximo. Esse valor máximo seria ou o valor de $N$ já pré-determinado, ou outro valor maior que viesse a minimizar a dispersão do estimador. De forma simultânea, uma janela de tamanho fixo $n$ seria utilizada para detectar rapidamente pontos de mudança na série temporal. Tais pontos de mudança poderiam ser causados tanto por uma alteração no valor de $H$, como por uma alteração na componente SRD presente, o que pode ser facilmente identificado (cf. (6)). Caso seja identificado uma alteração de $H$ e da componente $\mathrm{SRD}$, a janela $N$ seria reinicializada com seu valor mínimo e as condições revistas para o novo valor de $H$ estimado (mantendo-se fixa a estimativa da componente SRD) ou para a nova componente SRD identificada (mantendo-se fixa a estimativa inicial de $H$ para efeitos de inicialização do algoritmo).

O Algoritmo 1, abaixo apresentado, representa o mecanismo acima descrito através de um pseudocódigo em português estruturado.

O Apêndice A apresenta uma forma detalhada do mecanismo proposto, de 
forma a servir de ponto de partida para eventuais futuras implementações do mesmo. Cabe ressaltar que o mecanismo proposto não é definitivo, uma vez que não foi implementado ou testado de forma prática, mas baseia-se exclusivamente nas observações experimentais obtidas no presente trabalho.

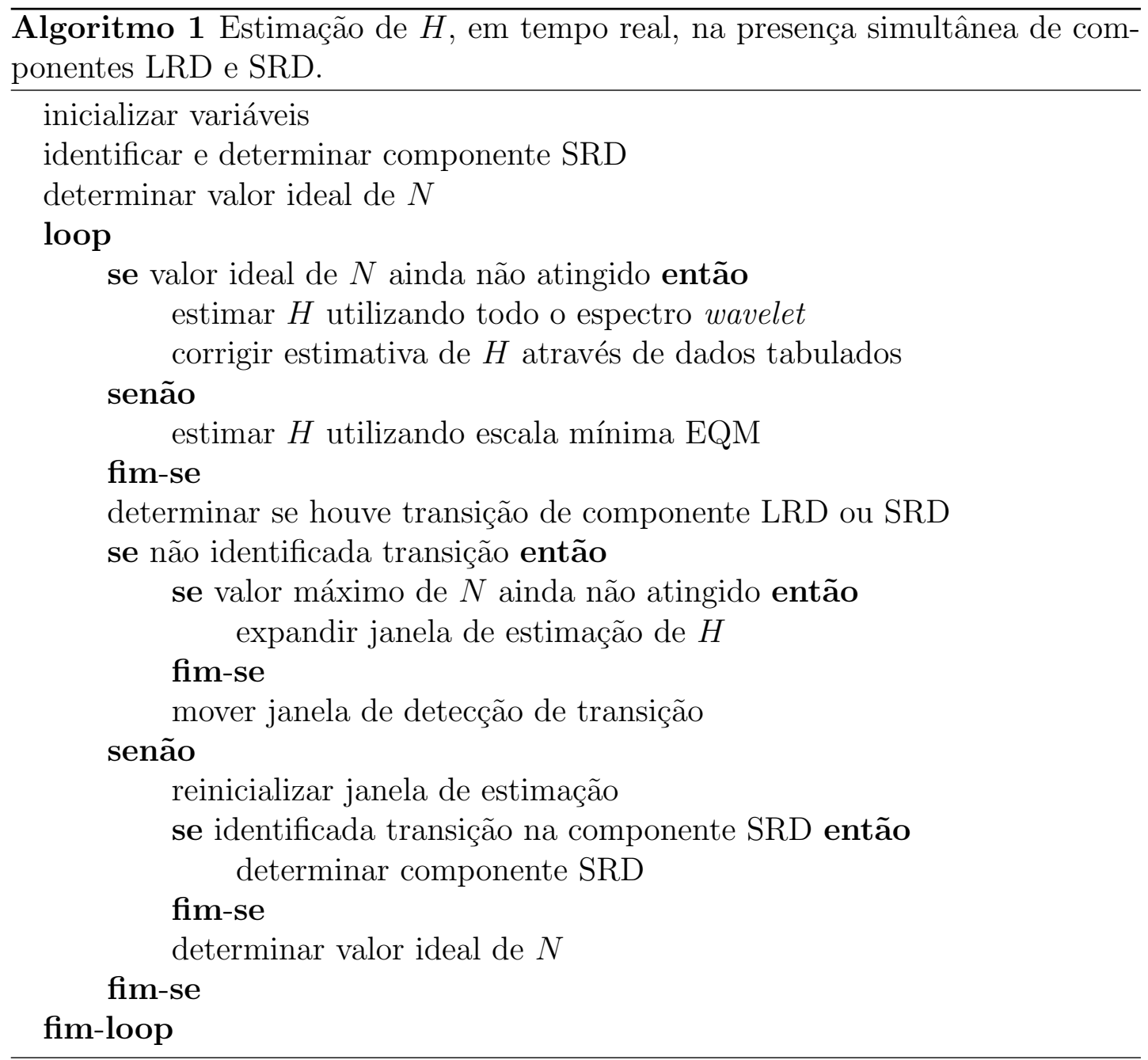




\subsection{Questões em aberto}

Os seguintes pontos, abaixo apresentados, merecem um estudo aprofundado para uma correta implementação do mecanismo de estimação proposto:

- Ao utilizar a DWPT para determinar a componente SRD (cf. (9)), qual é a melhor maneira de determinar a frequência média de tal componente?

- Ao realizar a estimação de $H \operatorname{com} j_{\min }=1$ e posteriormente corrigi-la (cf. (7)), até que ponto tal abordagem produz resultados com menor viés do que utilizando $j_{\min }=j_{\min }^{E Q M}$ ?

- Ao implementar o esquema de janela de estimação dupla (cf. (5)), qual é o valor de $n$ que otimiza a detecção de pontos de mudança e qual é o valor máximo de $N$ que pode ser utilizado sem que se comprometa a implementação em tempo real?

Não menos importantes, os pontos abaixo apresentados, uma vez esclarecidos, podem contribuir para uma otimização do mecanismo proposto:

- É possível automatizar a determinação da escala inicial do espectro que não sofre de influência de componentes SRD (cf. (2)), chamada de escala de transição?

- É possível comprovar que a utilização de $j_{\text {min }}$ igual à escala de transição (cf. (3) e (4)) produz estimativas com menor dispersão e viés, em substituição à utilização de $j_{\min }^{E Q M}$ ?

- Quais vantagens práticas e de implementação a estimação do espectro wavelet via DWPT (cf. (10)) poderia trazer para o método de Abry-Veitch? Como adaptar o método de Abry-Veitch para utilização do espectro wavelet estimado via DWPT?

- Que métrica poderia ser utilizada para determinação da árvore DWPT (cf. (11)) tal que se minimize os efeitos das componentes SRD na estimação do valor de $H$ ? 


\section{Parte III}

\section{Conclusões}




\section{Conclusões e trabalhos futuros}

O presente trabalho apresentou os efeitos potencialmente negativos da presença de componentes de dependência de curta duração na estimação do parâmetro de Hurst em séries de teletráfego e, através do estudo do espectro wavelet (seja através da transformada DWT - base do método de Abry-Veitch - seja através da transformada DWPT), foi possível obter diversos resultados práticos seja para aprimoramento do método de Abry-Veitch, seja para uma possível utilização do espectro wavelet obtido via DWPT para estimação do parâmetro de Hurst.

Os diversos resultados obtidos possibilitaram a elaboração de uma proposta de mecanismo alternativo para estimação em tempo real precisa das características de longa duração do tráfego, na presença de componentes de curta duração. Embora tal mecanismo não possa ser considerado como o resultado central desta pesquisa, uma vez que se trata tão simplesmente de uma proposta não implementada na prática (por não fazer parte do escopo deste trabalho), as conclusões e trabalhos futuros são apresentados justamente em torno desta proposta, uma vez que a mesma sumariza, de forma bastante completa e concisa, todos os principais resultados da pesquisa.

O mecanismo de estimação proposto baseia-se, de forma bastante simplificada, nos seguintes pontos:

- a estimação baseia-se em um esquema de janelas móveis duplas, sendo que uma janela maior é responsável pela estimação de $H$ e uma janela menor é responsável pela detecção de pontos de mudança da série, seja nas componentes SRD, seja nas componente LRD;

- a utilização da janela de detecção de pontos de mudança das componentes LRD ou SRD tende a permitir uma adaptação do algoritmo mais rápida e precisa;

- a DWPT é utilizada na identificação e determinação de eventuais compo- 
nentes SRD na série em estudo;

- a estimação pelo método de Abry-Veitch pode ser feita de forma precisa, na presença de SRD, utilizando-se de um número de amostras adequado (valor a ser determinado para cada série, dependendo do valor de $H$ e da componente SRD identificada) para o caso de utilização da escala mínima $j_{\min }=j_{\min }^{E Q M}$;

- a estimação pelo método de Abry-Veitch pode ser feita de forma igualmente precisa, na presença de SRD, utilizando-se de todo o espectro wavelet $\left(j_{\min }=1\right)$, desde que tal estimação tenha seu viés corrigido (correção a ser determinada para cada série, dependendo do valor de $H$ e da componente SRD identificada).

Para uma efetiva implementação do mecanismo, algumas questões ainda necessitam ser melhor compreendidas, como a implementação prática do mecanismo de determinação da componentes SRD via DWPT, ou a determinação (baseada em séries reais de teletráfego) do tamanho ideal das janelas de estimação e detecção de pontos de mudança. Adicionalmente, não foi possível comprovar, de forma rigorosa, que a utilização de $j_{\min }=1$ para a estimação, com a posterior correção do viés introduzido, produz estimativas satisfatórias, devendo tal comparação ser devidamente realizada.

De forma paralela à otimização do mecanismo proposto, o estudo para utilização da DWPT na estimativa do espectro wavelet, no método de Abry-Veitch, merece atenção em pesquisas futuras. Dentre as inúmeras árvores DWPT possíveis, a determinação da "árvore ideal", que minimize os efeitos da SRD na estimação de $H$, pode trazer um bom ganho nesta adaptação do método de Abry-Veitch.

Finalmente, uma linha de pesquisa promissora refere-se à identificação, no espectro wavelet, da chamada "escala de transição" (ou $j_{\text {trans }}$ ). Trata-se de uma escala a partir da qual não são mais observadas perturbações devido às componentes SRD e, teoricamente, poderia ser utilizada para excluir as escalas do espectro "contaminadas" pela dependência de curta duração e utilizar apenas as escalas com componente de longa duração para estimação de $H$. A constatação de tal escala foi visual, e uma mecanismo para determinação automatizada desta escala seria de grande valia. Mais ainda, a grande dispersão das estimativas de $H$ constatada para séries com um grande número de amostras pode ser potencialmente reduzida utilizando-se $j_{\text {min }}=j_{\text {trans }}$, já que isso forçaria uma estimativa com um número maior de escalas (já que, nesses casos, $j_{\text {trans }}<j_{\text {min }}^{E Q M}$ ). Tal constatação 
quanto à dispersão deve ser confirmada e o comportamento do viés neste caso devidamente analisado.

Apesar de não pertencer ao escopo do presente trabalho, a pesquisa dos tópicos abaixo relacionados pode contribuir para responder algumas das questões levantadas:

- o método de estimação de Abry-Veitch apresentou um bom desempenho sem a presença de componentes SRD. Uma alternativa para a estimação de $H$ em determinada série, na presença de SRD, seria a estimação de um modelo $\operatorname{ARFIMA}(\mathrm{p}, \mathrm{d}, \mathrm{q})$, de forma que tal modelo fosse utilizado para filtrar-se as componentes SRD da série em questão, para então proceder-se com a estimação de $H$ pelo método de Abry-Veitch;

- a análise espectral utilizando a DWPT poderia ser explorada para uma detecção eficaz de periodicidades e componentes SRD que degradam a estimação de $H$;

- métodos de estimação de $H$ correlatos ao método de Abry-Veitch merecem um estudo comparativo para averiguação de pontos fortes e fracos de cada método; como exemplo de método correlato pode-se citar o método descrito em [49, pág. 373], em que é formulada uma função de máxima verossimilhança diretamente em termos dos coeficientes wavelet. 


\section{Referências}

[1] LIMA, A. B. de. Proposta de uma Estratégia para Controle de Admissão de Conexões Baseado em Medições de Tráfego Agregado e Caracterização de Redes IP. Dissertação (Dissertação de Mestrado) - Escola Politécnica da USP, São Paulo, 2002.

[2] GROSSGLAUSER, M.; BOLOT, J.-C. On the relevance of long-range dependence in network traffic. IEEE/ACM Transactions on Networking, v. 7, n. 5, p. 629-640, Outubro 1999.

[3] LIMA, A. B. de. Contribuições à Modelagem de Teletráfego Fractal. Tese (Tese de Doutorado) — Escola Politécnica da USP, São Paulo, Brasil, 2008.

[4] LELAND, W. et al. On the self-similar nature of Ethernet traffic (extended version). IEEE/ACM Transactions on Networking, v. 2, n. 1, p. 1-15, Fevereiro 1994.

[5] PAXSON, V. Fast, approximate synthesis of fractional Gaussian noise for generating self-similar network traffic. Computer Communication review, v. 27, p. 5-18, Outubro 1997.

[6] TAQqU, M.; TEVEROVSKY, V.; WILlingER, W. Estimators for longrange dependence: An empirical study. Fractals, v. 3, p. 785-798, 1995.

[7] ABRY, P. et al. Wavelets for the analysis, estimation and synthesis of scaling data. In: PARK, K.; WILLINGER, W. (Ed.). Self Similar Network Traffic Analysis and Performance Evaluation. [S.l.]: John Wiley \& Sons, Inc., 2000. cap. 2, p. 39-88.

[8] TAQQU, M. S.; TEVEROVSKY, V. Robustness of Whittle-type estimates for time series with long-range dependence. [S.l.], 1997.

[9] TAQQU, M. S.; TEVEROVSKY, V. On estimating the intensity of longrange dependence in finite and infinite variance time series. In: ADLER, R. J.; FELDMAN, R. E.; TAQQU, M. S. (Ed.). A Practical Guide to Heavy Tails: Statistical Techniques and Applications. Boston: Birkhäuser, 1998. p. 177-217.

[10] ABRY, P. et al. Self-similarity and long-range dependence through the wavelets lens. In: DOUKHAN, P.; OPPENHEIM, G.; TAQQU, M. (Ed.). Theory and Applications of Long Range Dependence. Boston: Birkhäuser, 2002. p. $527-556$.

[11] DAVIE, B. S.; REKHTER, Y. MPLS: Technology and Applications. first. [S.l.]: Morgan Kaufmann, 2000.

[12] ROSEn, E.; VISWANATHAN, A.; CALLON, R. Internet RFC 3031: Multiprotocol Label Switching Architecture. 2001. Disponível em: <http://www. ietf .org/rfc/rfc3031.txt>. 
[13] MINEI, I.; LUCEK, J. MPLS-Enabled Applications: Emerging Developments and New Technologies. [S.l.]: John Wiley \& Sons, 2005.

[14] BRADEN, R.; CLARK, D.; SHENKER, S. Internet RFC 1633: Integrated Services in the Internet Architecture. 1994. Disponível em: <http: //www.ietf.org/rfc/rfc1633.txt>.

[15] NICHOLS, K. Internet RFC 2474: Definition of the Differentiated Services Field (DS field) in the IPv4 and IPv6 Headers. 1998. Disponível em: < http: //www. ietf.org/rfc/rfc2474.txt>.

[16] BLAKE, S. Internet RFC 2475: An Architecture for Differentiated Services. 1998. Disponível em: <http://www.ietf.org/rfc/rfc2475.txt>.

[17] ARMITAGE, G. Quality of Service in IP Networks. [S.l.]: MacMillan Technical Publishing, 2000.

[18] WANG, Z. Internet QoS: Architectures and Mechanisms for Quality of Service. first. [S.l.]: Morgan Kaufmann, 2001.

[19] QIU, J.; KNIGHTLY, E. W. Measurement-based admission control with aggregate traffic envelopes. IEEE/ACM Transactions on Networking, v. 9, n. 2, p. 199-210, Abril 2001. Disponível em: <http://citeseer.nj.nec.com/ qiu01measurementbased.htm $>$.

[20] ERLANG, A. K. The theory of probabilities and telephone conversation. Nyt Tidsskrift for Matematik B (first publication), v. 20, p. -, 1909. Disponível em: $<$ http://oldwww.com.dtu.dk/teletraffic/Erlang.html >.

[21] GUBNER, J. A. Probability and Random Processes for Electrical and Computer Engineers. Cambridge, UK: Cambridge University Press, 2006.

[22] PAXSON, V.; FLOYD, S. Wide-area traffic: The failure of Poisson modeling. IEEE/ACM Transactions on Networking, v. 3, n. 3, p. 226-244, Junho 1995.

[23] BATES, S.; MCLAUGHLIN, S. Testing the gaussian assumption for selfsimilar teletraffic models. In: IEEE Signal Processing Workshop on HigherOrder Statistics. [S.l.: s.n.], 1997. p. 444-447.

[24] KARASARIDIS, A.; HATZINAKOS, D. On the modeling of network traffic and fast simulation of rare events using stable self-similar processes. In: IEEE Signal Processing Workshop on Higher-Order Statistics (HOS). [S.l.: s.n.], 1997. p. 268-272.

[25] GARROPPO, R. G. et al. Testing $\alpha$-stable processes in modeling broadband teletraffic. In: IEEE International Conference on Communications, 2000 (ICC 2000). [S.l.: s.n.], 2000. v. 3, p. 1615-1619.

[26] KARASARIDIS, A.; HATZINAKOS, D. Network heavy traffic modeling using $\alpha$-stable self-similar processes. IEEE Transactions on Communications, v. 49, n. 7, p. 1203-1214, Julho 2001.

[27] KIM, S.; LEE, J. Y.; SUNG, D. K. A shifted gamma distribution model for long-range dependent internet traffic. IEEE Communications Letters, v. 7, n. 3, p. 124-126, Março 2003. 
[28] PETROPUlU, A. P.; YANG, X. Self-similarity and long-range dependence through the wavelet lens. In: BARNER, K. E.; ARCE, G. R. (Ed.). Nonlinear Signal and Image Processing - Theory, Methods, and Applications. [S.l.]: CRC Press, 2004.

[29] PARK, C. et al. Long-range dependence in a changing internet traffic mix. Computer Networks, v. 48, p. 401-422, 2005.

[30] STOEV, S. et al. On the wavelet spectrum diagnostic for hurst parameter estimation in the analysis of internet traffic. Computer Networks, v. 48, p. 423-445, Junho 2005.

[31] MELLO, F. L. de et al. Geração de séries auto-similares Gaussianas via wavelets para uso em simulações de tráfego. IEEE Latin America Transactions, v. 5, n. 1, p. 9-20, Março 2007. Disponível em: <http://www.ewh.ieee.org/reg/9/etrans/ieee/issues/vol5/ vol5issue1March2007/5TLA1_02LemosdeMello.pdf $>$.

[32] ABRY, P.; VEITCH, D.; FLANDRIN, P. Long-range dependence: Revisiting aggregation with wavelets. Journal of Time Series Analysis, v. 19, p. 253-266, 1998.

[33] VEITCH, D. N.; ABRY, P. A wavelet-based joint estimator of the parameters of long-range dependence. IEEE Transactions on Information Theory, v. 45, n. 3, p. 878-897, Abril 1999.

[34] ROUGHAN, M.; VEITCH, D.; ABRY, P. On-line estimation of the parameters of long-range dependence. In: IEEE Global Telecommunications Conference (Globecom'98). Sydney, Australia: [s.n.], 1998. v. 6, p. 3716-3721. Disponível em: <http://www.cubinlab.ee.unimelb.edu.au/ 〜arryl/Publications/on-line_Globecom98.pdf $>$.

[35] ROUGHAN, M.; VEITCH, D.; ABRY, P. Real-time estimation of the parameters of long-range dependence (extended version). IEEE/ACM Transactions on Networking, v. 8, n. 4, p. 467-478, Agosto 2000. Disponível em: <http://www.cubinlab.ee.unimelb.edu.au/ ${ }^{\sim}$ darryl/Publications/ on-lineToN.pdf $>$.

[36] ROUGHAN, M.; VEITCH, D. Measuring long-range dependence under changing traffic conditions. In: Annual Joint Conference of the IEEE Computer and Communications Societies (Infocom'99). Manhattan, NY: IEEE Computer Society Press, Los Alamitos, California, 1999. p. 1513-1521. Disponível em: <http://www.cubinlab.ee.unimelb.edu.au/〜darryl/Publications/ AVrobustness_camera.pdf $>$.

[37] ROUGHAN, M.; VEITCH, D. A study of the daily variation in the selfsimilarity of real data traffic. In: KEY, P.; SMITH, D. (Ed.). Proc. 16th International Teletraffic Congress (ITC-16). Edinburgh: Elsevier, Amsterdam, 1999. v. 3b, p. 67-76. Disponível em: <http://www.cubinlab.ee.unimelb. edu.au/〜darryl/Publications/diurnal_ITC16.pdf $>$.

[38] VEITCH, D. N.; ABRY, P. A statistical test for the time constancy of scaling exponents. IEEE Transactions on Signal Processing, v. 49, n. 10, p. 2325-2334, Outubro 2001. Disponível em: <http: //www. cubinlab.ee.unimelb.edu.au/ narryl/Publications/constancy_camera.pdf $>$. 
[39] BERAN, J. Statistics for Long-Memory Processes. [S.l.]: Chapman \& Hall, 1994.

[40] MANDELBROT, B. B. The Fractal Geometry of Nature. New York: WH Freeman, 1977.

[41] FALCONER, K. Fractal geometry, Mathematical Foundations and Applications. England: John Wiley \& Sons, Inc., 1990.

[42] FELDMANN, A.; GILBERT, A. C.; WILlinGER, W. Data networks as cascades: investigating the multifractal nature of internet wan traffic. Computer Communication review, v. 28, n. 4, p. 42-55, 1998.

[43] PARK, K.; WILLINGER, W. Self Similar Network Traffic And Performance Evaluation. [S.l.]: John Wiley \& Sons, Inc., 2000.

[44] RIEDI, R. H. et al. A multifractal wavelet model with application to network traffic. IEEE Transactions on Information Theory, v. 45, n. 3, p. 992-1018, Abril 1999.

[45] RIEDI, R. Multifractal processes. In: DOUKAN, P.; OPPENHEIM, G.; TAQQU, M. S. (Ed.). Theory and Applications of Long Range Dependence. Boston, MA: Birkhäuser, 2003.

[46] SPROTT, J. C. Chaos and Time-Series Analysis. New York: Oxford University Press, 2003.

[47] KLIMKE, A. Mandelbrot Set GUI (for MATLAB). 2003. Disponível em: $<$ http://matlabdb.mathematik.uni-stuttgart.de/index.jsp $>$.

[48] FRAME, M.; MANDELBROT, B. B.; NEGER, N. Natural Fractals, Class on Fractal Geometry offered by Yale University. 2006. Disponível em: $<$ http://classes.yale.edu/fractals/>.

[49] PERCIVAL, D. B.; WALDEN, A. T. Wavelet Methods for Time Series Analysis. [S.l.]: Cambridge University Press, 2000.

[50] CAPPé, O. et al. Long-range dependence and heavy-tail modeling for teletraffic data. IEEE Signal Processing Magazine, v. 19, n. 3, p. 14-27, Maio 2002 .

[51] TAQQU, M. S. Fractional brownian motion and long-range dependence. In: DOUKAN, P.; OPPENHEIM, G.; TAQQU, M. S. (Ed.). Theory and Applications of Long Range Dependence. Boston, MA: Birkhäuser, 2003. p. 5-38.

[52] HURST, H. E. Long-term storage capacity of reservoirs. Trans. Am. Soc. Civil Engineers, v. 116, p. 770-799, 1951.

[53] MANDELBROT, B. B.; NESS, J. V. Fractional brownian motions, fractional noises and applications. SIAM Rev., v. 10, p. 422-437, Fevereiro 1968.

[54] TSYBAKOV, B.; GEORGANAS, N. D. On self-similar traffic in atm queues: definitions, overflow probability bound, and cell delay distribution. IEEE Trans. Networking, v. 5, p. 397-409, Junho 1997. 
[55] MALlAT, S. A Wavelet Tour of Signal Processing. [S.l.]: Academic Press, 1999.

[56] ADDISON, P. S. The Illustrated Wavelet Transform Handbook. [S.1.]: Institute of Physics Publishing, 2002.

[57] DAUBECHIES, I. Orthonormal bases of compactly supported wavelets. Comm. Pure Appl. Math., v. 41, p. 909-996, 1988.

[58] DAUBECHIES, I. Ten Lectures on Wavelets. Philadelphia: SIAM, 1992.

[59] GROSSMANN, A.; MORLET, J. Decomposition of hardy functions into square integrable wavelets of constant shape. SIAM J. Math., v. 15, p. 723$736,1984$.

[60] COIFMAN, R.; MEYER, Y.; WICKERHAUSER, M. V. Wavelet analysis and signal processing. In: RUSKAI, M. B. et al. (Ed.). Wavelets and their applications. Boston, MA: Jones and Bartlett, 1992.

[61] FLANDRIN, P. Wavelet analysis and synthesis of fractional brownian motion. IEEE Transactions on Information Theory, v. 38, n. 2, p. 910-917, 1992.

[62] ABRY, P.; VEITCH, D. Wavelet analysis of long-range-dependent traffic. IEEE Transactions on Information Theory, v. 44, n. 1, p. 2-15, Janeiro 1998.

[63] WANG, Z. et al. Estimating hurst exponent with wavelet packet. ComputerAided Industrial Design and Conceptual Design, 2006. CAIDCD '06. '7th International Conference on, N/A, p. 1-4, Novembro 2006.

[64] KAISER, G. A Friendly Guide to Wavelets. Boston, Mass.: Birkhäuser, 1994.

[65] LUND, I. R. Contribuições à geração de tráfego fractal por meio da transformada wavelet. Dissertação (Dissertação de Mestrado) — Escola Politécnica da USP, São Paulo, Brasil, 2008.

[66] MISITI, M. et al. Wavelet Toolbox 4 - User's Guide. 3 Apple Hill Drive Natick, MA 01760-2098, October 2008.

[67] COIFMAN, R. R.; WICKERHAUSER, M. Entropy-based algorithms for best basis selection. In: IEEE Trans. on Inf. Theory. [S.l.: s.n.], 1992. v. 38, n. 2 , p. $713-718$.

[68] BOX, G. E. P.; JENKINS, G. M. Time Series Analysis - Forecasting and Control. [S.l.]: Holden-Day, 1976.

[69] TSAY, R. S. Analysis of Financial Time Series. 2. ed. Hoboken, New Jersey: John Wiley \& Sons, Inc., 2005.

[70] BROCKWELL, P. J.; DAVIS, R. A. Time series :theory and methods. $2^{\mathrm{a}}$. ed. New York: Springer-Verlag, 1991.

[71] BROCKWELL, P. J.; DAVIS, R. A. Introduction to Time Series and Forecasting. New York: Springer-Verlag, 1996.

[72] MORETtin, P. A.; TOLOI, C. M. C. Análise de Séries Temporais. [S.l.]: Editora Edgard Blücher Ltda., 2004. 
[73] ZIVOT, E.; WANG, J. Modeling Financial Time Series with S-PLUS. [S.l.]: Springer, 2003.

[74] PERCIVAL, D. B.; WALDEN, A. T. Spectral Analysis for Physical Applications. New York, NY: Cambridge University Press, 1993.

[75] STARK, H.; WOODS, J. W. Probability and Random Processes with Applications to Signal. 3. ed. Upper Saddle River, NY: Prentice Hall, 2002.

[76] PERCIVAL, D. B. An introduction to spectral analysis and wavelets. In: CIARLINI, P. et al. (Ed.). Advanced Mathematical Tools in Metrology. Singapore: World Scientific, 1994, (Advances in Mathematics for Applied Sciences, v. 16). p. $175-186$.

[77] GRANGER, C. W. J.; JOYEUX, R. An introduction to long-memory time series models and fractional differencing. Journal of Time Series Analysis, v. 1, p. 15-29, Outubro 1980.

[78] HOSKING, J. R. M. Fractional differencing. Biometrika, v. 68, p. 165-176, Outubro 1981.

[79] VEITCH, D.; TAQQU, M. S.; ABRY, P. Meaningful mra initialization for discrete time series. Signal Processing, v. 80, p. 1971-1983, 2000.

[80] PAXSON, V. Measurements and Analysis of End-to-End Internet Traffic. Tese (Doutorado) - N/A, 1997.

[81] MELLO, F. L. de. Estudo e Implementação de um Gerador de Tráfego com Dependência de Longa Duração. Dissertação (Tese de Mestrado) — Escola Politécnica da USP, São Paulo, Brasil, 2006.

[82] LIMA, A. B. de et al. A generator of teletraffic with long and short-range dependence. In: 12th Computer Aided Modeling and Design of Communication Links and Networks (CAMAD07) Workshop, part of the 18th Annual IEEE International Symposium on Personal, Indoor and Mobile Radio. Athens, Greece: [s.n.], 2007.

[83] LIMA, A. B. de et al. Introducing Short-Range Dependence in non-Gaussian 1/f-Type Teletraffic. [S.l.], 2006. 


\title{
Apêndice A - Pseudocódigo detalhado do mecanismo de estimação proposto
}

\begin{abstract}
A fim de facilitar futuras implementações do mecanismo proposto para estimação do parâmetro $H$, em tempo real, na presença simultânea de componentes LRD e SRD, o seguinte pseudocódigo detalhado é apresentado a partir da próxima página. Deve-se ressaltar que tal código trata-se de uma mera sugestão de implementação do mecanismo, sendo que o mesmo ainda não foi devidamente implementado e/ou validado com séries temporais de teletráfego real ou sintetizado.
\end{abstract}


Proposta de mecanismo de estimação de $H$, em tempo real, na presença simultânea de componentes LRD e SRD

Entrada: $n_{\text {inicial }}, N_{\max }$, viés $s_{\max }$, dispersão $o_{\max }, H_{\text {inic }}, x[i]$

Saída: $H_{\text {estim }}$

// Inicializar variáveis

$n \leftarrow n_{\text {inicial }}$

$N \leftarrow n_{\text {inicial }}$

$H_{\text {estim }} \leftarrow H_{\text {inicial }}$

janela_n $n_{\min } \leftarrow 1$

janela $\_n_{\max } \leftarrow n_{\text {inicial }}$

janela_N $N_{\text {min }} \leftarrow 1$

janela_N$N_{\max } \leftarrow n_{\text {inicial }}$

// Identificar e determinar componente SRD

$S R D_{\text {bool }} \leftarrow \operatorname{identSRD}\left(x\left[j a n e l a \_N_{\text {min }}:\right.\right.$ janela_ $\left.\left.N_{\text {max }}\right]\right)$

se $S R D_{b o o l}=$ verdadeiro então

$$
S R D_{\text {comp }} \leftarrow \operatorname{determSRD}\left(x\left[\text { janela_} N_{\min }: \text { janela } \_N_{\max }\right]\right)
$$

fim-se

// Determinar valor ideal de $N$

$N_{\text {ideal }} \leftarrow \operatorname{determ} N\left(S R D_{\text {comp }}, H_{\text {estim }}\right)$

loop

se $N<N_{\text {ideal }}$ então // Valor ideal de $N$ ainda não atingido

escala $_{\min } \leftarrow 1$

// Estimar $H$ utilizando todo o espectro wavelet

$H_{\text {estim }} \leftarrow \operatorname{estimarH}\left(x\left[j\right.\right.$ anela_ $N_{\min }:$ janela_N$\left.N_{\text {max }}\right]$, escalam $\left.a_{\text {min }}\right)$

// Corrigir estimativa de $H$ através de dados tabulados

$H_{\text {estim }} \leftarrow$ corrigirH $\left(H_{\text {estim }}, S R D_{\text {componente }}\right)$

senão // Valor ideal de $\mathrm{N}$ já atingido

// Estimar $H$ utilizando escala mínima ideal

escala $_{\text {min }} \leftarrow$ determEscalaEQM $\left(x\left[\right.\right.$ janela $\_N_{\text {min }}:$ janela_ $\left.\left.N_{\text {max }}\right]\right)$

$H_{\text {estim }} \leftarrow \operatorname{estimarH}\left(x\left[j a n e l a_{-} N_{\text {min }}:\right.\right.$ janela_ $\left.N_{\text {max }}\right]$,escal $\left.a_{\text {min }}\right)$

fim-se

// Determinar se houve transição de componente LRD ou SRD

$\left[\right.$ trans $_{\text {bool }}$, trans $\left._{\text {tipo }}\right] \leftarrow \operatorname{determTrans}\left(x\left[\right.\right.$ janela_n $n_{\text {min }}:$ janela_n $\left.\left.\left.n_{\text {max }}\right]\right)\right)$ 
se trans $_{b o o l}=$ falso então // Não identificada transição

se $N<N_{\text {máximo }}$ então // Valor máximo de $N$ ainda não atingido

// Expandir janela de estimação de $H$

$$
\begin{aligned}
& N \leftarrow N+1 \\
& \text { janela_N} N_{\text {max }} \leftarrow \text { janela_} N_{\text {max }}+1
\end{aligned}
$$

\section{fim-se}

// Mover janela de detecção de transição

janela_n $n_{\text {min }} \leftarrow$ janela_n $n_{\text {min }}+1$

janela_n$n_{\max } \leftarrow j a n e l a \_n_{\max }+1$

senão // Identificada transição

// Reinicializar janela de estimação

$N \leftarrow n_{\text {inicial }}$

janela_ $N_{\min } \leftarrow$ janela_n$n_{\text {min }}$

janela_N$N_{\max } \leftarrow$ janela_n$n_{\max }$

se trans $_{\text {tipo }}=\mathrm{SRD}$ então // Identificada transição na componente SRD

// Identificar e determinar componente SRD

$S R D_{\text {bool }} \leftarrow \operatorname{identSRD}\left(x\left[\right.\right.$ janela $\_N_{\text {min }}:$ janela $\left.\left.\_N_{\text {max }}\right]\right)$

se $S R D_{b o o l}=$ verdadeiro então

$$
S R D_{\text {comp }} \leftarrow \operatorname{determSRD}\left(x\left[j \text { anela_} N_{\text {min }}: \text { janel } \_\_N_{\text {max }}\right]\right)
$$

\section{fim-se}

\section{fim-se}

// Determinar do valor ideal de $N$

$N_{\text {ideal }} \leftarrow \operatorname{determ} N\left(S R D_{\text {comp }}, H_{\text {estim }}\right)$

\section{fim-se}

\section{fim-loop}

